\title{
Regioselective and Stereospecific Rhodium-Catalyzed Allylic Cyanomethylation with an Acetonitrile Equivalent: Construction of Acyclic $\beta$-Quaternary Stereogenic Nitriles
}

\author{
Mai-Jan Tom ${ }^{a}$ and P. Andrew Evans $*, a, b$ \\ a Department of Chemistry, Queen's University, 90 Bader Lane, Kingston ON, K7L 3N6, Canada \\ ${ }^{b}$ Xiangya School of Pharmaceutical Sciences, Central South University, Changsha 410013, \\ Hunan, P. R. of China
}

\section{Contents of Supporting Information:}

\begin{tabular}{|c|c|c|}
\hline 1. & General Information & $\mathrm{S} 2$ \\
\hline 2. & $\begin{array}{l}\text { Representative Experimental Procedure for the Preparation of the } \\
\text { (Triorganosilyl)acetonitrile Derivatives } \mathbf{1 b} \text {-d }\end{array}$ & S3 \\
\hline 3. & Spectral Data for (Triorganosilyl)acetonitrile Derivatives 1b-d & S3 \\
\hline 4. & $\begin{array}{l}\text { Representative Experimental Procedure for the Preparation of Alkyl Tertiary } \\
\text { Allylic Carbonates 2a-o }\end{array}$ & $\mathrm{S} 4$ \\
\hline 5. & Spectral Data for the Alkyl Tertiary Allylic Carbonates 2a-o & S4 \\
\hline 6. & Experimental Procedure for the Preparation of the Tertiary Allylic Alcohol 2p' & S9 \\
\hline 7. & $\begin{array}{l}\text { Representative Experimental Procedure for the Preparation of Aryl Tertiary } \\
\text { Allylic Carbonates } \mathbf{2 q - a a}\end{array}$ & $\mathrm{S} 10$ \\
\hline 8. & Spectral Data for the Aryl Tertiary Allylic Carbonates 2q-aa & $\mathrm{S} 11$ \\
\hline 9. & $\begin{array}{l}\text { Representative Experimental Procedure for the Regioselective Rhodium- } \\
\text { Catalyzed Allylic Cyanomethylation 3a-aa }\end{array}$ & $\mathrm{S} 14$ \\
\hline 10. & Spectral Data for the $\beta$-Quaternary Nitrile Products 3a-aa & S16 \\
\hline 11. & Stereospecific Rhodium-Catalyzed Allylic Cyanomethylation of $(R)-\mathbf{2 q}$ to $(R)-\mathbf{3 q}$ & $\mathrm{S} 26$ \\
\hline 12. & Derivations of the Enantioenriched Nitrile $(R)-\mathbf{3 q}$ to 5-7 & $\mathrm{S} 27$ \\
\hline 13. & References & $\mathrm{S} 29$ \\
\hline 14. & Copies of Proton and Carbon NMR Spectra & $\mathrm{S} 31$ \\
\hline 15. & HPLC Chromatograms & S140 \\
\hline
\end{tabular}




\section{General Information}

All reactions were carried out under an argon atmosphere in anhydrous solvent using commercially available reagents that were purchased and used as received. $(R)$-Linalool (97:3 er) was purchased from Aldrich and used directly. Tetrahydrofuran was freshly distilled from sodium benzophenone ketyl. Analytical thin layer chromatography (TLC) was performed on pre-coated $0.2 \mathrm{~mm}$ thick silica gel 60-F 254 plates (Merck); visualized using UV light and/or $\mathrm{KMnO}_{4}$ solution, followed by heating. All compounds were purified by flash chromatography using silica gel $60(40-63 \mu \mathrm{m}$, Silicycle) and gave spectroscopic data consistent with being $\geq 95 \%$ the assigned structure. Optical rotations $\left([\alpha]_{\mathrm{D}}^{\circ \mathrm{C}}\right)$ were measured on an Anton Parr MCP 200 polarimeter with a tungsten halogen lamp (589 nm) at the stated temperature (indicated in ${ }^{\circ} \mathrm{C}$ as superscript) using a $0.7 \mathrm{~mL}$ quartz cell of $100 \mathrm{~mm}$ length; solution concentrations (c) are given in $\mathrm{g} / 100 \mathrm{~mL}$. Melting points (uncorrected) were obtained from a Büchi M560 melting point instrument. ${ }^{1} \mathrm{H}$ NMR and ${ }^{13} \mathrm{C}$ NMR spectra were recorded on a Bruker Avance DRX-500 spectrometer in $\mathrm{CDCl}_{3}$ at ambient temperature; chemical shifts $(\delta)$ are given in ppm and calibrated using the signal of residual undeuterated solvent as internal reference for ${ }^{1} \mathrm{H} \mathrm{NMR}\left(\delta_{\mathrm{H}}=7.26 \mathrm{ppm}\right.$ for $\left.\mathrm{CDCl}_{3}\right)$ and using the signal of the deuterated solvent for ${ }^{13} \mathrm{C} \mathrm{NMR}\left(\delta_{\mathrm{C}}=77.16 \mathrm{ppm}\right.$ for $\left.\mathrm{CDCl}_{3}\right)$. ${ }^{1} \mathrm{H} \mathrm{NMR}$ data are reported as follows: chemical shift (multiplicity, 1st order spin system if available, coupling constant, integration). Coupling constants $(J)$ are reported in $\mathrm{Hz}$ and splitting patterns are designated using the following abbreviations: s (singlet), d (doublet), t (triplet), q (quartet), m (multiplet), br (broad), app. (apparent) and the appropriate combinations. ${ }^{13} \mathrm{C}$ NMR spectra with proton decoupling were described with the aid of an APT sequence, separating methylene and quaternary carbons (e, even), from methyl and methine carbons (o, odd). IR spectra were recorded on an Agilent Technologies Cary 630 FT-IR (ATR) spectrometer; wavenumbers (v) are given in $\mathrm{cm}^{-1}$; and the abbreviations W (weak, <33\%), m (medium, 33-66\%), s (strong, 67-95\%), vs (very strong, >95\%) and br (broad) are used to describe the relative intensities of the IR absorbance bands. Mass spectra were obtained through the Chemistry Department Mass Spectrometry Service at Queen's University. All liquid chromatographs were obtained on an Agilent 1200 series or an Agilent 1260 Infinity series HPLC equipped with a variable wavelength UV detector. 
2. Representative Experimental Procedure for the Preparation of the (Triorganosilyl)acetonitrile Derivatives 1b-d

$n$-Butyllithium ( $8.0 \mathrm{~mL}, 20 \mathrm{mmol} ; 2.5 \mathrm{M}$ in hexanes) was added dropwise to a stirred solution of acetonitrile $(1.0 \mathrm{~mL}, 20 \mathrm{mmol})$ in tetrahydrofuran $(40 \mathrm{~mL})$ at $-78^{\circ} \mathrm{C}$. The anion was allowed to form over $c a .30$ minutes, then tert-butyldimethylsilyl chloride $(3.0 \mathrm{~g}, 20 \mathrm{mmol})$ in tetrahydrofuran $(10 \mathrm{~mL})$ was added dropwise. The reaction was allowed to stir at $-78{ }^{\circ} \mathrm{C}$ for $\mathrm{ca}$. 1 hour before being warmed to room temperature (TLC control). The reaction mixture was then quenched by the addition of sat. aq $\mathrm{NH}_{4} \mathrm{Cl}$ and partitioned with diethyl ether. The organic phases were combined, dried (anhyd. $\mathrm{MgSO}_{4}$ ), filtered and concentrated in vacuo to afford the crude product. Purification by vacuum distillation afforded the 2-(tert-butyldimethylsilyl)acetonitrile (1d) (1.96 $\mathrm{g}, 63 \%)$ as a white solid.

\section{Spectra data for the (Triorganosilyl)acetonitrile Derivatives $1 \mathrm{~b}-\mathrm{d}$}

${ }_{\mathrm{TES}} \widehat{\mathrm{CN}}_{\mathrm{Color} \text { and State: Colorless oil. }} \begin{aligned} & \text { 2-(Triethylsilyl)acetonitrile (1b) } \\ & \end{aligned}$

${ }^{1} \mathbf{H}$ NMR $\left(500 \mathrm{MHz}, \mathrm{CDCl}_{3}\right) \delta 1.58(\mathrm{~s}, 2 \mathrm{H}), 1.00(\mathrm{t}, J=7.9 \mathrm{~Hz}, 9 \mathrm{H}), 0.72(\mathrm{q}, J=7.9 \mathrm{~Hz}, 6 \mathrm{H})$.

IR (Neat) 2955 (m), 2911 (w), 2877 (m), 2231 (m), 1458 (w), 1414 (w), 1240 (w), $1146(\mathrm{~m}), 1013$ (m), $764(\mathrm{~s}), 742(\mathrm{~s}), 718(\mathrm{~s}) \mathrm{cm}^{-1}$.

\section{TIPS $\widehat{C N}_{\mathrm{CN}}$ 2-(Triisopropylsilyl)acetonitrile (1c) \\ Color and State: Colorless oil.}

${ }^{1} \mathbf{H}$ NMR $\left(500 \mathrm{MHz}, \mathrm{CDCl}_{3}\right) \delta 1.58(\mathrm{~s}, 2 \mathrm{H}), 1.26-1.19(\mathrm{~m}, 3 \mathrm{H}), 1.11$ (d, J=7.1 Hz, 18H).

${ }^{13}$ C NMR (125 MHz, $\mathrm{CDCl}_{3}$ ) $\delta 119.89$ (e), 18.37 (o), 11.03 (o), -3.21 (e).

IR (Neat) 2943 (s), 2891 (m), 2867 (s), 2231 (m), 1462 (m), 1387 (w), 1248 (w), 1148 (m), 999 (m), 881 (vs), 734 (s), 706 (s) $\mathrm{cm}^{-1}$.

HRMS (EI, $\mathrm{M}^{+}$) calcd for $\mathrm{C}_{11} \mathrm{H}_{23} \mathrm{NSi}$ 197.1600, found 197.1609.

\section{TBS $\widehat{C N N}^{2-(t e r t-B u t y l d i m e t h y l s i l y l) a c e t o n i t r i l e ~(1 d) ~}{ }^{2}$ \\ Color and State: White solid; $\mathbf{m p}=77-78^{\circ} \mathrm{C}$; Lit. $\mathbf{m p}=71-72{ }^{\circ} \mathrm{C}$.}

${ }^{1}$ H NMR $\left(500 \mathrm{MHz}, \mathrm{CDCl}_{3}\right) \delta 1.58(\mathrm{~s}, 2 \mathrm{H}), 0.95$ (s, 9H), $0.18(\mathrm{~s}, 6 \mathrm{H})$.

${ }^{13}$ C NMR (125 MHz, CDCl 3 ) $\delta 119.49$ (e), 26.13 (o), 16.76 (e), 1.09 (e), -6.13 (o). 
IR (Neat) 2952 (m), 2930 (m), 2858 (m), 2232 (m), 1467 (m), 1392 (w), 1253 (m), 1146 (m), $841(\mathrm{~s}), 825$ (s), $810(\mathrm{~s}), 790(\mathrm{~s}), 708(\mathrm{~m}) \mathrm{cm}^{-1}$.

HRMS (ESI, $[\mathrm{M}+\mathrm{H}]^{+}$) calcd for $\mathrm{C}_{8} \mathrm{H}_{18} \mathrm{NSi}$ 156.1203, found 156.1203.

\section{Representative Experimental Procedure for the Preparation of Alkyl Tertiary Allylic} Carbonates 2a-o

Lithium bis(trimethylsilyl)amide $(22.0 \mathrm{~mL}, 22.0 \mathrm{mmol} ; 1 \mathrm{M}$ solution in tetrahydrofuran) was added dropwise to a stirring solution of 3-methyl-5-phenylpent-1-en-3-ol (3.5 g, $20 \mathrm{mmol})$ in tetrahydrofuran $(40 \mathrm{~mL})$ at $0{ }^{\circ} \mathrm{C}$. The anion was allowed to form over $c a .30$ minutes, then methyl chloroformate $(2.0 \mathrm{~mL}, 26.0 \mathrm{mmol})$ was added dropwise and the reaction was allowed to slowly warm to room temperature and stirred until completion (TLC control). The reaction mixture was then quenched by the addition of sat. aq $\mathrm{NH}_{4} \mathrm{Cl}$ and extracted with diethyl ether. The organic phases were combined, dried (anhyd. $\mathrm{MgSO}_{4}$ ), filtered and concentrated in vacuo to afford the crude product. Purification by flash column chromatography (silica gel, eluting with 2-8\% diethyl ether/hexane) afforded the tertiary allylic carbonate $\mathbf{2 a}(3.90 \mathrm{~g}, 83 \%)$ as a colorless oil.

\section{Spectral Data for the Alkyl Tertiary Allylic Carbonates 2a-o}

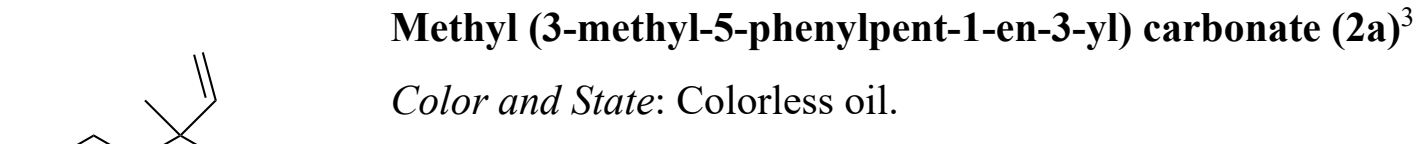

$\mathrm{Ph}_{\mathrm{OCO}_{2} \mathrm{Me}}{ }^{1} \mathbf{H}$ NMR $\left(500 \mathrm{MHz}, \mathrm{CDCl}_{3}\right) \delta$ 7.29-7.26 (m, 2H), 7.19-7.17 (m, 3H), 6.05 (dd, $J=17.5,11.0 \mathrm{~Hz}, 1 \mathrm{H}), 5.27(\mathrm{~d}, J=17.5 \mathrm{~Hz}, 1 \mathrm{H}), 5.23$ (d, $J=11.1 \mathrm{~Hz}, 1 \mathrm{H}), 3.73$ (s, 3H), 2.69$2.61(\mathrm{~m}, 2 \mathrm{H}), 2.22-2.06(\mathrm{~m}, 2 \mathrm{H}), 1.64(\mathrm{~s}, 3 \mathrm{H})$.

IR (Neat) 3025 (w), 2953 (w), 2852 (w), 1742 (s), 1645 (w), 1439 (m), 1257 (vs), 1170 (m), 1087 (m), $928(\mathrm{~m}), 875(\mathrm{~m}), 791(\mathrm{~m}), 697(\mathrm{~s}) \mathrm{cm}^{-1}$.

\footnotetext{
Methyl (2-methyl-1-phenylbut-3-en-2-yl) carbonate (2b) ${ }^{3}$

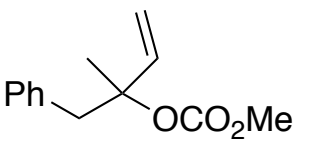

Color and State: Colorless oil.

${ }^{1} \mathbf{H}$ NMR $\left(500 \mathrm{MHz}, \mathrm{CDCl}_{3}\right) \delta$ 7.30-7.21 (m, 3H), 7.20-7.18 (m, 2H), 6.04 $(\mathrm{dd}, J=17.5,11.0 \mathrm{~Hz}, 1 \mathrm{H}), 5.20$ (d, $J=10.7 \mathrm{~Hz}, 1 \mathrm{H}), 5.17(\mathrm{~d}, J=17.4 \mathrm{~Hz}, 1 \mathrm{H}), 3.73(\mathrm{~s}, 3 \mathrm{H}), 3.17$ $\left(\mathrm{d}, \mathrm{A}\right.$ of $\left.\mathrm{AB}, J_{\mathrm{AB}}=13.7 \mathrm{~Hz}, 1 \mathrm{H}\right), 3.05\left(\mathrm{~d}, \mathrm{~B}\right.$ of $\left.\mathrm{AB}, J_{\mathrm{AB}}=13.7 \mathrm{~Hz}, 1 \mathrm{H}\right), 1.55(\mathrm{~s}, 3 \mathrm{H})$.
} 
IR (Neat) 3029 (w), 2954 (w), 2851 (w), 1741 (s), 1643 (w), 1439 (m), 1261 (s), 1242 (s), 1165 (m), 1084 (s), 920 (m), 859 (m), 791 (m), 699 (s) $\mathrm{cm}^{-1}$.

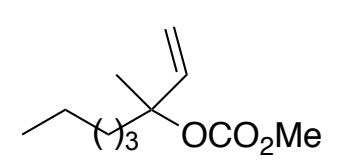

\section{Methyl (3-methyloct-1-en-3-yl) carbonate (2c)}

Color and State: Colorless oil.

${ }^{1} \mathbf{H}$ NMR $\left(500 \mathrm{MHz}, \mathrm{CDCl}_{3}\right) \delta 6.00(\mathrm{dd}, J=17.5,11.0 \mathrm{~Hz}, 1 \mathrm{H}), 5.19(\mathrm{dd}, J$ $=18.0,0.7 \mathrm{~Hz}, 1 \mathrm{H}), 5.16(\mathrm{dd}, J=11.1,0.7 \mathrm{~Hz}, 1 \mathrm{H}), 3.71(\mathrm{~s}, 3 \mathrm{H}), 1.84-1.74(\mathrm{~m}, 2 \mathrm{H}), 1.56(\mathrm{~s}, 3 \mathrm{H})$, 1.35-1.24 (m, 6H), $0.88(\mathrm{t}, J=7.0 \mathrm{~Hz}, 3 \mathrm{H})$.

${ }^{13}$ C NMR (125 MHz, $\left.\mathrm{CDCl}_{3}\right) \delta 154.04$ (e), 141.25 (o), 114.16 (e), 84.84 (e), 54.16 (o), 39.99 (e), 32.12 (e), 23.31 (e), 23.12 (o), 22.66 (e), 14.12 (o).

IR (Neat) 2955 (w), 2932 (w), 2864 (w), 1745 (s), 1644 (w), 1439 (m), 1261 (s), 1137 (m), 1074 (w), $926(\mathrm{~m}), 881(\mathrm{~m}), 792(\mathrm{~m}) \mathrm{cm}^{-1}$.

HRMS (ESI, $[\mathrm{M}+\mathrm{Na}]^{+}$) calcd for $\mathrm{C}_{11} \mathrm{H}_{20} \mathrm{NaO}_{3} 223.1305$, found 223.1314.

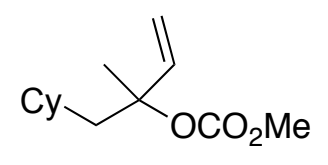

\section{1-Cyclohexyl-2-methylbut-3-en-2-yl methyl carbonate (2d)}

Color and State: Colorless oil.

${ }^{1} \mathbf{H}$ NMR $\left(500 \mathrm{MHz}, \mathrm{CDCl}_{3}\right) \delta 6.00(\mathrm{dd}, J=17.5,10.9 \mathrm{~Hz}, 1 \mathrm{H}), 5.20(\mathrm{~d}, J=$ $17.5 \mathrm{~Hz}, 1 \mathrm{H}), 5.15(\mathrm{~d}, J=10.9 \mathrm{~Hz}, 1 \mathrm{H}), 3.71(\mathrm{~s}, 3 \mathrm{H}), 1.80-1.58(\mathrm{~m}, 7 \mathrm{H}), 1.57(\mathrm{~s}, 3 \mathrm{H}), 1.48-1.39$ $(\mathrm{m}, 1 \mathrm{H}), 1.27-1.18(\mathrm{~m}, 2 \mathrm{H}), 1.13$ (pentet of triplets, $J=12.3,3.1 \mathrm{~Hz}, 1 \mathrm{H}), 0.96$ (app. qd, $J=12.0$, $2.6 \mathrm{~Hz}, 2 \mathrm{H})$.

${ }^{13}$ C NMR (125 MHz, $\left.\mathrm{CDCl}_{3}\right) \delta 153.99$ (e), 141.74 (o), 113.74 (e), 85.13 (e), 54.11 (o), 46.95 (e), 34.76 (e), 34.69 (e), 33.54 (o), 26.46 (e), 26.32 (e), 23.96 (o).

IR (Neat) 2921 (m), 2849 (w), 1744 (s), 1643 (w), 1440 (m), 1254 (vs), 1168 (m), 1083 (m), 923 (m), $878(\mathrm{~m}), 791(\mathrm{~m}) \mathrm{cm}^{-1}$.

HRMS (ESI, $[\mathrm{M}+\mathrm{Na}]^{+}$) calcd for $\mathrm{C}_{13} \mathrm{H}_{22} \mathrm{NaO}_{3} 249.1461$, found 249.1457.

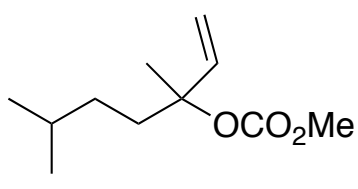

\section{3,6-Dimethylhept-1-en-3-yl methyl carbonate (2e) ${ }^{3}$}

Color and State: Colorless oil.

${ }^{1}$ H NMR $\left(500 \mathrm{MHz}, \mathrm{CDCl}_{3}\right) \delta 5.99(\mathrm{dd}, J=17.5,11.0 \mathrm{~Hz}, 1 \mathrm{H}), 5.20(\mathrm{dd}$, $J=17.4,0.7 \mathrm{~Hz}, 1 \mathrm{H}), 5.17(\mathrm{dd}, J=10.9,0.9 \mathrm{~Hz}, 1 \mathrm{H}), 3.71(\mathrm{~s}, 3 \mathrm{H}), 1.82\left(\mathrm{dt}, \mathrm{A}\right.$ of $\mathrm{ABX}_{2}, J_{\mathrm{AB}}=$ $\left.13.3 \mathrm{~Hz}, J_{\mathrm{AX}}=8.3 \mathrm{~Hz}, 1 \mathrm{H}\right), 1.78\left(\mathrm{dt}, \mathrm{B}\right.$ of $\left.\mathrm{ABX}_{2}, J_{\mathrm{AB}}=13.6 \mathrm{~Hz}, J_{\mathrm{BX}}=8.3 \mathrm{~Hz}, 1 \mathrm{H}\right), 1.56(\mathrm{~s}, 3 \mathrm{H})$, 1.50 (nonet, $J=6.6 \mathrm{~Hz}, 1 \mathrm{H}), 1.22-1.17(\mathrm{~m}, 2 \mathrm{H}), 0.88(\mathrm{~d}, J=6.7 \mathrm{~Hz}, 6 \mathrm{H})$. 
IR (Neat) 2955 (m), 2870 (w), 1745 (s), 1645 (w), 1439 (m), 1371 (w), 1266 (s), 1241 (s), 1170 (m), $1074(\mathrm{~m}), 923(\mathrm{~m}), 872(\mathrm{~m}), 792(\mathrm{~m}) \mathrm{cm}^{-1}$.

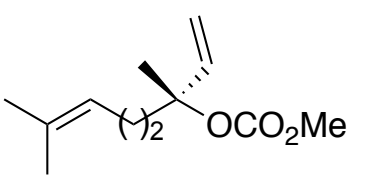

(R)-3,7-Dimethylocta-1,6-dien-3-yl methyl carbonate $((R)-(2 f))^{4}$

Color and State: Colorless oil.

$[\alpha]_{\mathrm{D}}^{20}-10.1\left(c=1.0, \mathrm{CHCl}_{3}\right)$; Lit. $[\alpha]_{\mathrm{D}}^{20}-3.2\left(c=1.0, \mathrm{CHCl}_{3}\right)$

Chiral HPLC analysis of the 4-methoxybenzoyl-protected alcohol (CHIRALCEL OJ-H column), 99.4:0.6 hexane/isopropanol at $0.7 \mathrm{~mL} / \mathrm{min}$. flow rate; $t_{\mathrm{R}}(S)$-enantiomer (minor) $=9.5 \mathrm{~min} ., t_{\mathrm{R}}(R)$ enantiomer $($ major $)=10.8$ min.; $94 \%$ ee.

${ }^{1} \mathbf{H}$ NMR $\left(500 \mathrm{MHz}, \mathrm{CDCl}_{3}\right) \delta 6.00(\mathrm{dd}, J=17.5,11.0 \mathrm{~Hz}, 1 \mathrm{H}), 5.21(\mathrm{dd}, J=17.6 \mathrm{~Hz}, 0.7 \mathrm{~Hz}$, $1 \mathrm{H}), 5.18(\mathrm{dd}, J=11.8 \mathrm{~Hz}, 0.7 \mathrm{~Hz}, 1 \mathrm{H}), 5.10-5.06(\mathrm{~m}, 1 \mathrm{H}), 3.71(\mathrm{~s}, 3 \mathrm{H}), 2.00$ (app q. $J=8.0 \mathrm{~Hz}$, 2H), 1.90-1.77 (m, 2H), 1.67 (s, 3H), 1.59 (s, 3H), 1.57 (s, 3H).

IR (Neat) 2969 (w), 2918 (w), 2856 (w), 1745 (s), 1644 (w), 1439 (m), 1265 (s), 1242 (s), 1169 (m), 1081 (m), 991 (w), 925 (m), 879 (m), 791 (m) $\mathrm{cm}^{-1}$.

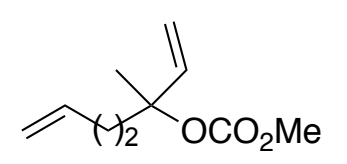

\section{Methyl (3-methylhepta-1,6-dien-3-yl) carbonate (2g)}

Color and State: Colorless oil.

${ }^{1} \mathbf{H}$ NMR $\left(500 \mathrm{MHz}, \mathrm{CDCl}_{3}\right) \delta 5.99(\mathrm{dd}, J=17.5,11.0 \mathrm{~Hz}, 1 \mathrm{H}), 5.80$ (ddt, $J$ $=17.0,10.3,6.6 \mathrm{~Hz}, 1 \mathrm{H}), 5.21(\mathrm{dd}, J=17.5,0.7 \mathrm{~Hz}, 1 \mathrm{H}), 5.19(\mathrm{dd}, J=11.0,0.6 \mathrm{~Hz}, 1 \mathrm{H}), 5.02$ (app. dq, $J=17.1,1.7 \mathrm{~Hz}, 1 \mathrm{H}), 4.95$ (app. dq, $J=10.2,1.5 \mathrm{~Hz}, 1 \mathrm{H}), 3.72$ (s, 3H), 2.12-2.07 (m, 2H), 1.96-1.84 (m, 2H), 1.58 (s, 3H).

IR (Neat) 3079 (w), 2980 (w), 2954 (w), 2852 (w), 1744 (s), 1641 (w), 1440 (m), 1263 (vs), 1238 (s), $1170(\mathrm{~m}), 1079(\mathrm{~m}), 912$ (m), $876(\mathrm{~m}), 791(\mathrm{~m}) \mathrm{cm}^{-1}$.

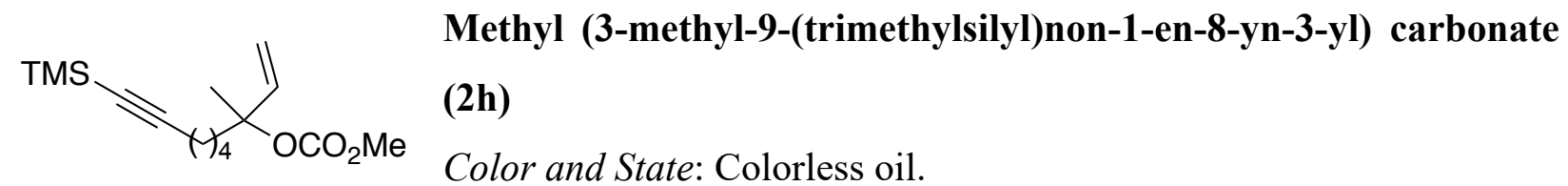

${ }^{1} \mathbf{H}$ NMR $\left(500 \mathrm{MHz}, \mathrm{CDCl}_{3}\right) \delta 5.99(\mathrm{dd}, J=17.5,11.0 \mathrm{~Hz}, 1 \mathrm{H}), 5.20(\mathrm{~d}, J=17.8 \mathrm{~Hz}, 1 \mathrm{H}), 5.17$ $(\mathrm{d}, J=11.3 \mathrm{~Hz}, 1 \mathrm{H}), 3.71(\mathrm{~s}, 3 \mathrm{H}), 2.22(\mathrm{t}, J=6.9 \mathrm{~Hz}, 2 \mathrm{H}), 1.84\left(\mathrm{dt}, \mathrm{A}\right.$ of $\mathrm{ABX}_{2}, J_{\mathrm{AB}}=13.8 \mathrm{~Hz}$, $\left.J_{\mathrm{AX}}=8.4 \mathrm{~Hz}, 1 \mathrm{H}\right), 1.79\left(\mathrm{dt}, \mathrm{B}\right.$ of $\left.\mathrm{ABX}_{2}, J_{\mathrm{AB}}=14.1 \mathrm{~Hz}, J_{\mathrm{BX}}=8.2 \mathrm{~Hz}, 1 \mathrm{H}\right), 1.56(\mathrm{~s}, 3 \mathrm{H}), 1.55-1.49$ $(\mathrm{m}, 2 \mathrm{H}), 1.46-1.40(\mathrm{~m}, 2 \mathrm{H}), 0.14(\mathrm{~s}, 9 \mathrm{H})$. 
${ }^{13}$ C NMR (125 MHz, $\left.\mathrm{CDCl}_{3}\right) \delta 154.02$ (e), 141.05 (o), 114.36 (e), 107.29 (e), 84.86 (e), 84.66 (e), 54.20 (o), 39.36 (e), 28.77 (e), 23.15 (o), 22.84 (e), 19.83 (e), 0.28 (o).

IR (Neat) 2954 (w), 2865 (w), 2173 (w), 1745 (s), 1644 (w), 1440 (m), 1258 (s), 1250 (s), 1171 (m), 1092 (m), 940 (m), 837 (s), 792 (m), 758 (m), 697 (w) cm'.

HRMS (ESI, $[\mathrm{M}+\mathrm{H}]^{+}$) calcd for $\mathrm{C}_{15} \mathrm{H}_{27} \mathrm{O}_{3} \mathrm{Si} 283.1724$, found 283.1724.

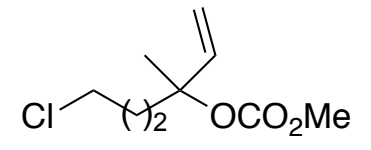

\section{6-Chloro-3-methylhex-1-en-3-yl methyl carbonate (2i)}

Color and State: Yellow oil.

$=17.5 \mathrm{~Hz}, 1 \mathrm{H}), 5.20(\mathrm{~d}, J=10.9 \mathrm{~Hz}, 1 \mathrm{H}), 3.72(\mathrm{~s}, 3 \mathrm{H}), 3.54(\mathrm{t}, J=6.5 \mathrm{~Hz}, 2 \mathrm{H}), 2.05-1.88(\mathrm{~m}$, 2H), 1.87-1.80 (m, 2H), $1.59(\mathrm{~s}, 3 \mathrm{H})$.

${ }^{13}$ C NMR (125 MHz, $\mathrm{CDCl}_{3}$ ) $\delta 153.94$ (e), 140.64 (o), 114.70 (e), 84.07 (e), 54.30 (o), 45.10 (e), 37.41 (e), 27.11 (e), 23.42 (o).

IR (Neat) 2988 (w), 2957 (w), 2863 (w), 1743 (s), 1644 (w), 1440 (m), 1260 (s), 1170 (m), 1085 (m), $931(\mathrm{~m}), 883(\mathrm{~m}), 791(\mathrm{~m}) \mathrm{cm}^{-1}$.

HRMS (ESI, $[\mathrm{M}+\mathrm{Na}]^{+}$) calcd for $\mathrm{C}_{9} \mathrm{H}_{15}{ }^{35} \mathrm{ClNaO}_{3} 229.0602$, found 229.0598.

\section{Color and State: Colorless oil.}

$\mathrm{PivO}_{2} \mathrm{OCO}_{2} \mathrm{Me} \quad{ }^{1} \mathbf{H}$ NMR $\left(500 \mathrm{MHz}, \mathrm{CDCl}_{3}\right) \delta 5.98(\mathrm{dd}, J=17.5,11.0 \mathrm{~Hz}, 1 \mathrm{H}), 5.22(\mathrm{~d}$, $J=17.5 \mathrm{~Hz}, 1 \mathrm{H}), 5.20(\mathrm{~d}, J=11.0 \mathrm{~Hz}, 1 \mathrm{H}), 4.05(\mathrm{t}, J=6.5 \mathrm{~Hz}, 2 \mathrm{H}), 3.72(\mathrm{~s}, 3 \mathrm{H}), 1.95-1.80(\mathrm{~m}$, 2H), 1.71-1.63 (m, 2H), $1.58(\mathrm{~s}, 3 \mathrm{H}), 1.19(\mathrm{~s}, 9 \mathrm{H})$.

${ }^{13}$ C NMR (125 MHz, $\mathrm{CDCl}_{3}$ ) $\delta 178.64$ (e), 153.93 (e), 140.72 (o), 114.67 (e), 84.15 (e), 64.23 (e), 54.27 (o), 38.89 (e), 36.33 (e), 27.33 (o), 23.25 (o), 23.23 (e).

IR (Neat) 2959 (w), 2874 (w), 1746 (s), 1725 (s), 1645 (w), 1440 (m), 1268 (s), 1149 (s), 1035 (m), $939(\mathrm{~m}), 792(\mathrm{~m}) \mathrm{cm}^{-1}$.

HRMS (ESI, [M+Na] $]^{+}$) calcd for $\mathrm{C}_{14} \mathrm{H}_{24} \mathrm{NaO}_{5}$ 295.1516, found 295.1513. 


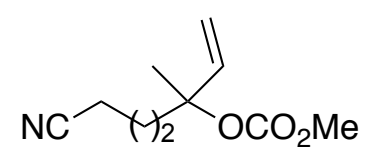

\section{6-Cyano-3-methylhex-1-en-3-yl methyl carbonate (2k)}

Color and State: Colorless oil.

${ }^{1}$ H NMR $\left(500 \mathrm{MHz}, \mathrm{CDCl}_{3}\right) \delta 5.96(\mathrm{dd}, J=17.5,11.0 \mathrm{~Hz}, 1 \mathrm{H}), 5.23(\mathrm{~d}, J$

$=17.8 \mathrm{~Hz}, 1 \mathrm{H}), 5.22(\mathrm{~d}, J=11.0 \mathrm{~Hz}, 1 \mathrm{H}), 3.72(\mathrm{~s}, 3 \mathrm{H}), 2.36(\mathrm{t}, J=7.1 \mathrm{~Hz}, 2 \mathrm{H}), 2.05-1.96(\mathrm{~m}$, 1H), 1.94-1.85 (m, 1H), 1.77-1.68 (m, 2H), 1.59 (s, 3H).

${ }^{13}$ C NMR (125 MHz, $\left.\mathrm{CDCl}_{3}\right) \delta 153.85$ (e), 140.26 (o), 119.40 (e), 114.93 (e), 83.68 (e), 54.35

(o), 38.96 (e), 23.41 (o), 20.05 (e), 17.40 (e).

IR (Neat) 3089 (w), 2956 (w), 2852 (w), 2246 (w), 1742 (s), 1644 (w), 1439 (m), 1263 (vs), 1173 (m), 1089 (m), 934 (m), $888(\mathrm{~m}), 791(\mathrm{~m}) \mathrm{cm}^{-1}$.

HRMS (ESI, $[\mathrm{M}+\mathrm{H}]^{+}$) calcd for $\mathrm{C}_{10} \mathrm{H}_{16} \mathrm{NO}_{3}$ 198.1125, found 198.1129.

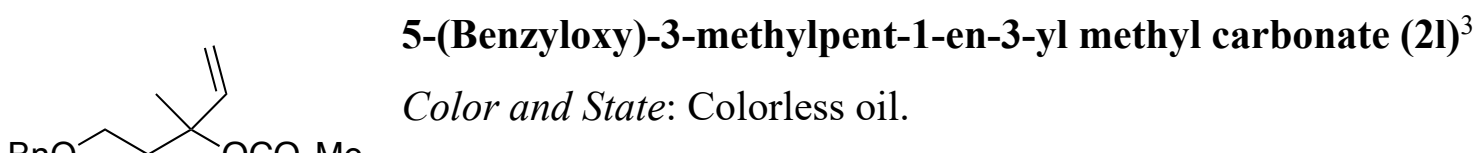

$\mathrm{BnO}_{\mathrm{OCO}_{2} \mathrm{Me}}{ }^{1} \mathbf{H} \mathbf{N M R}\left(500 \mathrm{MHz}, \mathrm{CDCl}_{3}\right) \delta$ 7.36-7.27 (m, 5H), $6.01(\mathrm{dd}, J=17.5,11.0$ $\mathrm{Hz}, 1 \mathrm{H}), 5.23(\mathrm{~d}, J=17.5 \mathrm{~Hz}, 1 \mathrm{H}), 5.18(\mathrm{~d}, J=11.0 \mathrm{~Hz}, 1 \mathrm{H}), 4.48(\mathrm{~s}, 2 \mathrm{H}), 3.68(\mathrm{~s}, 3 \mathrm{H}), 3.59$ (dt, $\mathrm{A}$ of $\left.\mathrm{ABX}_{2}, J_{\mathrm{AB}}=10.0 \mathrm{~Hz}, J_{\mathrm{AX}}=6.5 \mathrm{~Hz}, 1 \mathrm{H}\right), 3.58\left(\mathrm{dt}, \mathrm{B}\right.$ of $\mathrm{ABX}_{2}, J_{\mathrm{AB}}=10.0 \mathrm{~Hz}, J_{\mathrm{BX}}=6.5 \mathrm{~Hz}$, $1 \mathrm{H}), 2.23\left(\mathrm{dt}, \mathrm{A}\right.$ of $\left.\mathrm{ABX}_{2}, J_{\mathrm{AB}}=13.4 \mathrm{~Hz}, J_{\mathrm{AX}}=6.7 \mathrm{~Hz}, 1 \mathrm{H}\right), 2.20\left(\mathrm{dt}, \mathrm{B}\right.$ of $\mathrm{ABX}_{2}, J_{\mathrm{AB}}=13.5 \mathrm{~Hz}$, $\left.J_{\mathrm{BX}}=6.7 \mathrm{~Hz}, 1 \mathrm{H}\right), 1.61(\mathrm{~s}, 3 \mathrm{H})$.

IR (Neat) 3088 (w), 2988 (w), 2954 (w), 2860 (w), 1743 (s), 1643 (w), 1439 (m), 1260 (vs), $1168(\mathrm{~m}), 1093$ (s), 928 (m), 877 (m), 790 (m), 735 (s), $696(\mathrm{~s}) \mathrm{cm}^{-1}$.

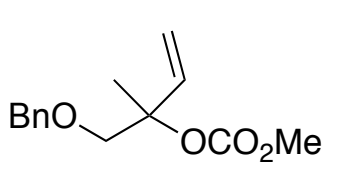

\section{1-(Benzyloxy)-2-methylbut-3-en-2-yl methyl carbonate $(2 \mathrm{~m})^{3}$} Color and State: Colorless oil.

${ }^{1} \mathbf{H}$ NMR $\left(500 \mathrm{MHz}, \mathrm{CDCl}_{3}\right) \delta$ 7.36-7.27 (m, 5H), $6.06(\mathrm{dd}, J=17.6,11.0$ $\mathrm{Hz}, 1 \mathrm{H}), 5.28(\mathrm{dd}, J=17.6,0.4 \mathrm{~Hz}, 1 \mathrm{H}), 5.25(\mathrm{dd}, J=11.1,0.5 \mathrm{~Hz}, 1 \mathrm{H}), 4.61\left(\mathrm{~d}, \mathrm{~A}\right.$ of $\mathrm{AB}, J_{\mathrm{AB}}=$ $12.3 \mathrm{~Hz}, 1 \mathrm{H}), 4.56\left(\mathrm{~d}, \mathrm{~B}\right.$ of $\left.\mathrm{AB}, J_{\mathrm{AB}}=12.1 \mathrm{~Hz}, 1 \mathrm{H}\right), 3.72(\mathrm{~s}, 3 \mathrm{H}), 3.67\left(\mathrm{~d}, \mathrm{~A}\right.$ of $\mathrm{AB}, J_{\mathrm{AB}}=10.0 \mathrm{~Hz}$, $1 \mathrm{H}), 3.58\left(\mathrm{~d}, \mathrm{~B}\right.$ of $\left.\mathrm{AB}, J_{\mathrm{AB}}=9.9 \mathrm{~Hz}, 1 \mathrm{H}\right), 1.62(\mathrm{~s}, 3 \mathrm{H})$.

IR (Neat) 3088 (w), 2991 (w), 2953 (w), 2859 (w), 1744 (s), 1643 (w), 1439 (m), 1257 (vs), 1091 (s), $928(\mathrm{~m}), 875(\mathrm{~m}), 791(\mathrm{~m}), 735$ (m), 697 (s) $\mathrm{cm}^{-1}$. 
$\searrow_{\mathrm{OCO}_{2} \mathrm{Me}}$

1-((4-Methoxybenzyl)oxy)-2-methylbut-3-en-2-yl methyl carbonate (2n)

Color and State: Colorless oil.

${ }^{1}$ H NMR $\left(500 \mathrm{MHz}, \mathrm{CDCl}_{3}\right) \delta$ 7.26-7.23 (m, 2H), 6.89-6.86 (m, 2H), $6.05(\mathrm{dd}, J=17.7,11.0 \mathrm{~Hz}$, $1 \mathrm{H}), 5.27(\mathrm{~d}, J=17.5 \mathrm{~Hz}, 1 \mathrm{H}), 5.23(\mathrm{dd}, J=11.1,0.4 \mathrm{~Hz}, 1 \mathrm{H}), 4.54\left(\mathrm{~d}, \mathrm{~A}\right.$ of $\mathrm{AB}, J_{\mathrm{AB}}=11.9 \mathrm{~Hz}$, $1 \mathrm{H}), 4.49\left(\mathrm{~d}, \mathrm{~B}\right.$ of $\left.\mathrm{AB}, J_{\mathrm{AB}}=11.8 \mathrm{~Hz}, 1 \mathrm{H}\right), 3.81(\mathrm{~s}, 3 \mathrm{H}), 3.72(\mathrm{~s}, 3 \mathrm{H}), 3.63\left(\mathrm{~d}, \mathrm{~A}\right.$ of $\mathrm{AB}, J_{\mathrm{AB}}=9.9$ $\mathrm{Hz}, 1 \mathrm{H}), 3.54\left(\mathrm{~d}, \mathrm{~B}\right.$ of $\left.\mathrm{AB}, J_{\mathrm{AB}}=9.9 \mathrm{~Hz}, 1 \mathrm{H}\right), 1.59(\mathrm{~s}, 3 \mathrm{H})$.

${ }^{13}$ C NMR (125 MHz, $\mathrm{CDCl}_{3}$ ) $\delta 159.34$ (e), 153.97 (e), 138.67 (o), 130.25 (e), 129.38 (o), 115.79 (e), 113.88 (o), 83.57 (e), 74.46 (e), 73.27 (e), 55.39 (o), 54.28 (o), 20.95 (o).

IR (Neat) 2996 (w), 2953 (w), 2858 (w), 2838 (w), 1744 (s), 1641 (w), 1611 (m), 1512 (m), 1440 (m), 1247 (vs), 1174 (m), 1083 (s), 1032 (s), 929 (m), 874 (m), 818 (m), 790 (m) cm . $^{-1}$.

HRMS (ESI, [M+Na] $]^{+}$) calcd for $\mathrm{C}_{15} \mathrm{H}_{20} \mathrm{O}_{5} \mathrm{Na} 303.1203$, found 303.1202.

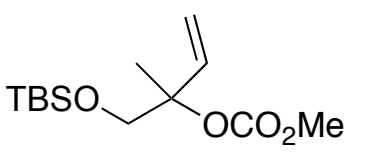

1-((tert-Butyldimethylsilyl)oxy)-2-methylbut-3-en-2-yl

methyl carbonate (20)

Color and State: Colorless oil.

${ }^{1}$ H NMR $\left(500 \mathrm{MHz}, \mathrm{CDCl}_{3}\right) \delta 6.03(\mathrm{dd}, J=17.6,11.1 \mathrm{~Hz}, 1 \mathrm{H}), 5.25(\mathrm{dd}, J=17.5,0.7 \mathrm{~Hz}, 1 \mathrm{H})$, $5.22(\mathrm{dd}, J=10.9,0.7 \mathrm{~Hz}, 1 \mathrm{H}), 3.80\left(\mathrm{~d}, \mathrm{~A}\right.$ of $\left.\mathrm{AB}, J_{\mathrm{AB}}=10.2 \mathrm{~Hz}, 1 \mathrm{H}\right), 3.71(\mathrm{~s}, 3 \mathrm{H}), 3.66(\mathrm{~d}, \mathrm{~B}$ of $\left.\mathrm{AB}, J_{\mathrm{AB}}=10.2 \mathrm{~Hz}, 1 \mathrm{H}\right), 1.56(\mathrm{~s}, 3 \mathrm{H}), 0.89(\mathrm{~s}, 9 \mathrm{H}), 0.05(\mathrm{~s}, 6 \mathrm{H})$.

${ }^{13}$ C NMR (125 MHz, $\left.\mathrm{CDCl}_{3}\right) \delta 154.05$ (e), 138.78 (o), 115.66 (e), 84.25 (e), 68.04 (e), 54.24 (o), 25.92 (o), 20.48 (o), 18.40 (e), -5.33 (o).

IR (Neat) 2954 (w), 2930 (w), 2857 (w), 1747 (s), 1645 (w), 1440 (m), 1261 (s), 1109 (s), 1096 (s), $936(\mathrm{~m}), 834(\mathrm{~s}), 774(\mathrm{~s}), 667(\mathrm{~m}) \mathrm{cm}^{-1}$.

HRMS (ESI, [M+Na $]^{+}$) calcd for $\mathrm{C}_{13} \mathrm{H}_{26} \mathrm{NaO}_{4} \mathrm{Si} 297.1493$, found 297.1495.

\section{Experimental Procedure for the Preparation of the Tertiary Allylic Alcohol 2p'}

Vinylmagnesium bromide $(7.43 \mathrm{~mL}, 5.20 \mathrm{mmol}$; $0.7 \mathrm{M}$ in tetrahydrofuran) was added dropwise to a stirred solution of 1-(4-methoxyphenyl)ethenone $(0.60 \mathrm{~g}, 4.00 \mathrm{mmol})$ in tetrahydrofuran (4 $\mathrm{mL}$ ) at $0{ }^{\circ} \mathrm{C}$. The resulting solution was allowed to stir for $c a .3$ hours (TLC control). The reaction mixture was then quenched by the addition of sat. aq $\mathrm{NH}_{4} \mathrm{Cl}$ and extracted with diethyl ether. The organic phases were combined, washed with brine, dried (anhyd. $\mathrm{MgSO}_{4}$ ), filtered and 
concentrated in vacuo to afford the crude product. Purification by flash column chromatography (silica gel, eluting with $10-20 \%$ diethyl ether/hexane) afforded the tertiary allylic alcohol $\mathbf{2 p}(0.613$ g, $86 \%$ ) as a yellow oil.

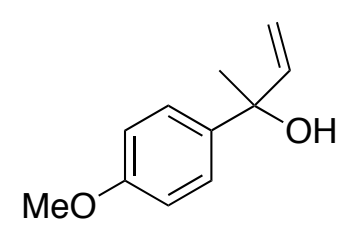

\section{2-(4-Methoxyphenyl)but-3-en-2-ol (2p' $)^{4}$}

Color and State: Yellow oil.

${ }^{1} \mathbf{H}$ NMR $\left(500 \mathrm{MHz}, \mathrm{CDCl}_{3}\right) \delta$ 7.41-7.38 (m, 2H), 6.89-6.86 (m, 2H), 6.16 (dd, $J=17.3,10.7 \mathrm{~Hz}, 1 \mathrm{H}), 5.28$ (dd, $J=17.2,1.1 \mathrm{~Hz}, 1 \mathrm{H}), 5.13$ (dd, $J=$ 10.5, $1.1 \mathrm{~Hz}, 1 \mathrm{H}), 3.80(\mathrm{~s}, 3 \mathrm{H}), 1.83(\mathrm{~s}, 1 \mathrm{H}), 1.64(\mathrm{~s}, 3 \mathrm{H})$.

IR (Neat) 3443 (br, w), 3082 (w), 2975 (w), 2835 (w), 1667 (w), 1640 (w), 1607 (m), 1508 (s), 1361 (w), 1244 (s), 1173 (s), 1029 (s), 917 (s), 873 (m), 829 (vs), 733 (w), 701 (w) cm cm $^{-1}$

\section{Representative Experimental Procedure for the Preparation of Aryl Tertiary Allylic Carbonates 2q-aa}

Method A: ${ }^{n}$ Butyllithium (3.36 mL, $8.40 \mathrm{mmol} ; 2.5 \mathrm{M}$ in hexanes) was added dropwise to a stirring solution of 2-p-tolylbut-3-en-2-ol $(1.43 \mathrm{~g}, 8.80 \mathrm{mmol})$ in tetrahydrofuran $(16.80 \mathrm{~mL})$ at $-78{ }^{\circ} \mathrm{C}$. The anion was allowed to form over $c a .30$ minutes, then di-tert-butyl dicarbonate $(1.75 \mathrm{~g}, 8.00$ $\mathrm{mmol})$ in tetrahydrofuran $(4.20 \mathrm{~mL})$ was added dropwise and the reaction was allowed to slowly warm to $4{ }^{\circ} \mathrm{C}$ and stirred for $c a$. 16 hours. The reaction mixture was then diluted with diethyl ether and water then extracted with diethyl ether. The organic phases were combined, dried (anhyd. $\mathrm{Na}_{2} \mathrm{SO}_{4}$ ), filtered and concentrated in vacuo to afford the crude product. Purification by flash column chromatography (silica gel, neutralized with 5\% triethylamine, eluting with 2-6\% diethyl ether/hexane) afforded the tertiary allylic carbonate $\mathbf{2 q}(1.66 \mathrm{~g}, 79 \%)$ as a pale yellow oil.

Method B: Potassium hydride (561.0 mg, $4.2 \mathrm{mmol} ; 30 \mathrm{wt} \%$ in mineral oil) was washed with hexanes $(5 \mathrm{~mL})$ for 20 seconds and the hexane removed after the sodium hydride had settled to the bottom of the flask (three times). Tetrahydrofuran $(6.4 \mathrm{~mL})$ was then added to the flask and the resulting suspension was cooled to $-78^{\circ} \mathrm{C}$ with stirring. 2-(4-Bromophenyl)but-3-en-2-ol (99.9 $\mathrm{mg}, 4.4 \mathrm{mmol}$ ) was added dropwise via tared syringe to the mixture and the anion was allowed to form over ca. 30 minutes, then di-tert-butyl dicarbonate $(0.93 \mathrm{~mL}, 4 \mathrm{mmol})$ in tetrahydrofuran $(1.6$ $\mathrm{mL}$ ) was added dropwise. The reaction was allowed to slowly warm to $4{ }^{\circ} \mathrm{C}$ and stirred for $c a .16$ 
hours. The reaction mixture was diluted with diethyl ether and water then extracted with diethyl ether. The organic phases were combined, dried (anhyd. $\mathrm{Na}_{2} \mathrm{SO}_{4}$ ), filtered and concentrated in vacuo to afford the crude product. Purification by flash column chromatography (silica gel, neutralized with $5 \%$ triethylamine, eluting with $2 \%$ diethyl ether/hexane) afforded the tertiary allylic carbonate $2 \mathbf{u}(1.13 \mathrm{~g}, 86 \%)$ as a yellow oil.

\section{Spectral Data for the Aryl Tertiary Allylic Carbonates 2q-aa}

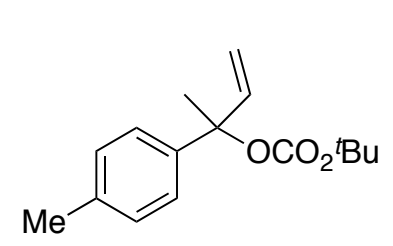

tert-Butyl (2-(p-tolyl)but-3-en-2-yl) carbonate (2q) ${ }^{5}$

Color and State: Pale yellow oil. (Method A)

${ }^{1} \mathbf{H}$ NMR $\left(500 \mathrm{MHz}, \mathrm{CDCl}_{3}\right) \delta 7.27(\mathrm{~d}, J=7.6 \mathrm{~Hz}, 2 \mathrm{H}), 7.14(\mathrm{~d}, J=8.0$

$\mathrm{Hz}, 2 \mathrm{H}), 6.33$ (dd, $J=17.5,10.8 \mathrm{~Hz}, 1 \mathrm{H}), 5.27$ (d, $J=17.4 \mathrm{~Hz}, 1 \mathrm{H}), 5.25$

(d, $J=10.8 \mathrm{~Hz}, 1 \mathrm{H}), 2.33$ (s, 3H), $1.86(\mathrm{~s}, 3 \mathrm{H}), 1.42(\mathrm{~s}, 9 \mathrm{H})$.

IR (Neat) 3092 (w), 2979 (w), 2933 (w), 1742 (s), 1641 (w), 1367 (m), 1274 (s), 1252 (s), 1153 (vs), 1093 (s), 924 (m), $850(\mathrm{~m}), 815(\mathrm{~s}), 790(\mathrm{~m}), 694(\mathrm{w}) \mathrm{cm}^{-1}$.

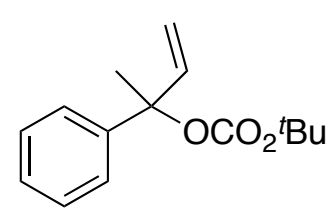

tert-Butyl (2-phenylbut-3-en-2-yl) carbonate (2r) $)^{5}$

Color and State: Pale yellow oil. (Method A)

${ }^{1} \mathbf{H}$ NMR $\left(500 \mathrm{MHz}, \mathrm{CDCl}_{3}\right) \delta$ 7.40-7.37 (m, 2H), 7.36-7.32 (m, 2H), 7.27$7.24(\mathrm{~m}, 1 \mathrm{H}), 6.34(\mathrm{dd}, J=17.4,10.8 \mathrm{~Hz}, 1 \mathrm{H}), 5.30-5.26(\mathrm{~m}, 2 \mathrm{H}), 1.87$ (s,

$3 \mathrm{H}), 1.41(\mathrm{~s}, 9 \mathrm{H})$.

IR (Neat) 3089 (w), 2980 (w), 2935 (w), 1741 (s), 1641 (w), 1368 (m), 1275 (s), 1252 (s), 1152 (s), $1096(\mathrm{~s}), 925(\mathrm{~m}), 847(\mathrm{~s}), 791(\mathrm{~m}), 761(\mathrm{~m}), 697(\mathrm{~s}) \mathrm{cm}^{-1}$.

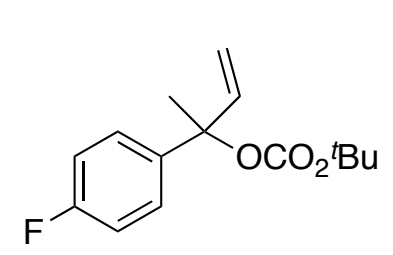

tert-Butyl (2-(4-fluorophenyl)but-3-en-2-yl) carbonate (2s) ${ }^{6}$ Color and State: Colorless oil. (Method A)

${ }^{1} \mathbf{H}$ NMR (500 MHz, $\left.\mathrm{CDCl}_{3}\right) \delta$ 7.38-7.34 (m, 2H), 7.04-6.99 (m, 2H), 6.31 (dd, $J=17.4,11.0 \mathrm{~Hz}, 1 \mathrm{H}), 5.27(\mathrm{dd}, J=10.9,0.8 \mathrm{~Hz}, 1 \mathrm{H}), 5.26$ (d, $J=$ $17.5,0.6 \mathrm{~Hz}, 1 \mathrm{H}), 1.86(\mathrm{~s}, 3 \mathrm{H}), 1.41(\mathrm{~s}, 9 \mathrm{H})$.

IR (Neat) 3090 (w), 2981 (w), 2936 (w), 1742 (s), 1641 (w), 1603 (w), 1509 (m), 1368 (m), 1275 (s), 1227 (s), 1152 (vs), 1089 (s), 926 (m), 832 (s), 791 (m), $694(\mathrm{w}) \mathrm{cm}^{-1}$. 


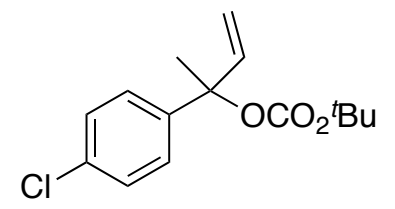

tert-Butyl (2-(4-chlorophenyl)but-3-en-2-yl) carbonate $(2 t)^{5}$

Color and State: Yellow oil. (Method $A)$

${ }^{1}$ H NMR $\left(500 \mathrm{MHz}, \mathrm{CDCl}_{3}\right) \delta$ 7.33-7.29 (m, 4H), 6.30 (dd, $J=17.3,10.9$

Hz, 1H), 5.28 (d, $J=10.8 \mathrm{~Hz}, 1 \mathrm{H}), 5.27$ (d, $J=17.7 \mathrm{~Hz}, 1 \mathrm{H}), 1.85$ (s, 3H), 1.42 (s, 9H).

IR (Neat) 3090 (w), 2980 (w), 2936 (w), 1741 (s), 1641 (w), 1491 (m), 1368 (m), 1275 (s), 1252 (s), 1153 (s), 1091 (vs), 1011 (s), 926 (m), 824 (s), 791 (m), 731 (m), 688 (w) cm cm $^{-1}$.

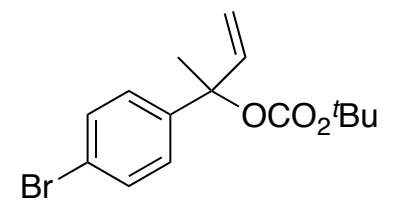

\section{2-(4-Bromophenyl)but-3-en-2-yl tert-butyl carbonate $(2 \mathrm{u})^{5}$}

Color and State: Yellow oil. (Method B)

${ }^{1} \mathbf{H}$ NMR $\left(500 \mathrm{MHz}, \mathrm{CDCl}_{3}\right) \delta$ 7.47-7.44 (m, 2H), 7.27-7.25 (m, 2H), 6.30 (dd, $J=17.3,10.9 \mathrm{~Hz}, 1 \mathrm{H}), 5.28(\mathrm{dd}, J=10.8,0.5 \mathrm{~Hz}, 1 \mathrm{H}), 5.27$ (d, $J=$ 17.4 Hz, 1H), $1.84(\mathrm{~s}, 3 \mathrm{H}), 1.42(\mathrm{~s}, 9 \mathrm{H})$.

IR (Neat) 3090 (w), 2979 (w), 2933 (w), 1741 (vs), 1641 (w), 1487 (m), 1368 (m), 1277 (s), 1252 (s), 1152 (vs), 1091 (s), 1007 (s), 926 (m), 848 (s), 820 (s), 791 (m), 719 (m) cm'.

tert-Butyl (2-(4-trifluoromethyl)phenyl)but-3-en-2-yl) carbonate (2v) $\overbrace{\mathrm{OCO}_{2} \mathrm{Bu}}^{1}$ Color and State: Colorless oil. (Method B)

${ }^{1} \mathbf{H}$ NMR $\left(500 \mathrm{MHz}, \mathrm{CDCl}_{3}\right) \delta$ 7.61-7.59 (m, 2H), 7.51-7.49 (m, 2H), $6.32(\mathrm{dd}, J=17.4,10.8 \mathrm{~Hz}, 1 \mathrm{H}), 5.32(\mathrm{dd}, J=10.8,0.6 \mathrm{~Hz}, 1 \mathrm{H}), 5.31$ (d, $J=17.4 \mathrm{~Hz}, 1 \mathrm{H}), 1.87(\mathrm{~s}, 3 \mathrm{H}), 1.42(\mathrm{~s}, 9 \mathrm{H})$.

${ }^{13}$ C NMR (125 MHz, $\left.\mathrm{CDCl}_{3}\right) \delta 151.56$ (e), 148.00 (e), 140.41 (o), 129.63 (e, q, ${ }^{2} J_{\mathrm{CF}}=32.5 \mathrm{~Hz}$ ), 125.62 (o), $125.45\left(\mathrm{o}, \mathrm{q},{ }^{3} J_{\mathrm{CF}}=3.7 \mathrm{~Hz}\right), 124.25\left(\mathrm{e}, \mathrm{q},{ }^{1} J_{\mathrm{CF}}=272.1 \mathrm{~Hz}\right), 116.07$ (e), $83.29(\mathrm{e}), 82.40$ (e), 27.91 (o), 25.94 (o).

${ }^{19}$ F NMR $\left(471 \mathrm{MHz}, \mathrm{CDCl}_{3}\right) \delta-62.53(\mathrm{~s})$.

IR (Neat) 3095 (w), 2982 (w), 2937 (w), 1743 (s), 1641 (w), 1619 (w), 1369 (m), 1325 (s), 1277 (s), 1254 (s), 1157 (s), 1120 (vs), 1094 (s), 1073 (s), 1014 (s), 929 (m), 836 (s), 792 (m), 705 (m) $\mathrm{cm}^{-1}$.

HRMS (ESI, $[\mathrm{M}+\mathrm{Na}]^{+}$) calcd for $\mathrm{C}_{16} \mathrm{H}_{19} \mathrm{~F}_{3} \mathrm{NaO}_{3} 339.1179$, found 339.1172. 
2-(Benzo $[d][1,3]$ dioxol-5-yl)but-3-en-2-yl tert-butyl carbonate $(2 \mathrm{w})^{5}$

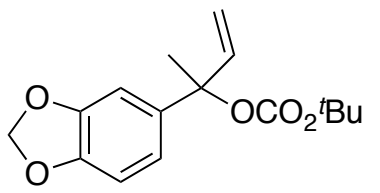

Color and State: Pale yellow oil. (Method A)

${ }^{1} \mathrm{H}$ NMR $\left(500 \mathrm{MHz}, \mathrm{CDCl}_{3}\right) \delta 6.89(\mathrm{~d}, J=1.7 \mathrm{~Hz}, 1 \mathrm{H}), 6.86(\mathrm{dd}, J=8.2$,

$1.9 \mathrm{~Hz}, 1 \mathrm{H}), 6.76(\mathrm{~d}, J=8.0 \mathrm{~Hz}, 1 \mathrm{H}), 6.29$ (dd, $J=17.5,10.8 \mathrm{~Hz}, 1 \mathrm{H})$,

$5.94(\mathrm{~s}, 2 \mathrm{H}), 5.26(\mathrm{dd}, J=17.5,0.7 \mathrm{~Hz}, 1 \mathrm{H}), 5.25$ (dd, $J=10.8,0.8 \mathrm{~Hz}, 1 \mathrm{H}), 1.84$ (s, 3H), 1.42 (s, 9H).

IR (Neat) 3088 (w). 2980 (w), 2927 (w), 2899 (w), 1740 (s), 1640 (w), 1504 (m), 1486 (m), 1435 (m), 1368 (m), 1276 (s), 1239 (vs), 1149 (s), 1088 (s), 1035 (s), 925 (m), 862 (m), 810 (s), 699 (m) $\mathrm{cm}^{-1}$.

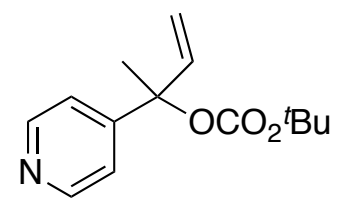

tert-Butyl (2-(pyridin-4-yl)but-3-en-2-yl) carbonate (2x)

Color and State: Pale yellow oil. (Method A)

${ }^{1} \mathbf{H}$ NMR $\left(500 \mathrm{MHz}, \mathrm{CDCl}_{3}\right) \delta 8.58-8.57(\mathrm{~m}, 2 \mathrm{H}), 7.28-7.27(\mathrm{~m}, 2 \mathrm{H}), 6.28$

(dd, $J=17.4,10.8 \mathrm{~Hz}, 1 \mathrm{H}), 5.33(\mathrm{dd}, J=10.9,0.5 \mathrm{~Hz}, 1 \mathrm{H}), 5.32(\mathrm{~d}, J=$

$17.5 \mathrm{~Hz}, 1 \mathrm{H}), 1.84(\mathrm{~s}, 3 \mathrm{H}), 1.42(\mathrm{~s}, 9 \mathrm{H})$.

${ }^{13}$ C NMR (125 MHz, $\mathrm{CDCl}_{3}$ ) $\delta 152.87$ (e), 151.46 (e), 150.07 (o), 139.68 (o), 120.15 (o), 116.51 (e), 82.60 (e), 82.57 (e), 27.88 (o), 25.59 (o).

IR (Neat) 3085 (w), 2980 (w), 2935 (w), 1742 (s), 1641 (w), 1596 (m), 1410 (m), 1368 (m), 1277 (vs), 1253 (s), 1153 (s), 1104 (s), 1068 (s), 993 (m), 928 (m), 851 (m), 819 (s), 791 (m), 706 (m), $680(\mathrm{~m}) \mathrm{cm}^{-1}$.

HRMS (ESI, $[\mathrm{M}+\mathrm{H}]^{+}$) calcd for $\mathrm{C}_{14} \mathrm{H}_{20} \mathrm{NO}_{3} 250.1438$, found 250.1427 .

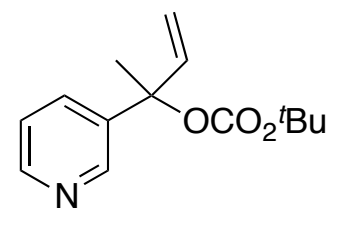

tert-Butyl (2-(pyridin-3-yl)but-3-en-2-yl) carbonate (2y)

Color and State: Pale yellow oil. (Method A)

${ }^{1} \mathbf{H}$ NMR $\left(500 \mathrm{MHz}, \mathrm{CDCl}_{3}\right) \delta 8.66(\mathrm{dd}, J=2.4,0.7 \mathrm{~Hz}, 1 \mathrm{H}), 8.52(\mathrm{dd}, J=$ $4.8,1.6 \mathrm{~Hz}, 1 \mathrm{H}$ ), 7.69 (ddd, $J=8.0,2.4,1.6 \mathrm{~Hz}, 1 \mathrm{H}), 7.27$ (ddd, $J=8.1,4.7$, $0.9 \mathrm{~Hz}, 1 \mathrm{H}), 6.32$ (dd, $J=17.5,10.9 \mathrm{~Hz}, 1 \mathrm{H}), 5.33$ (dd, $J=11.0,0.6 \mathrm{~Hz}, 1 \mathrm{H}), 5.30$ (dd, $J=18.1$, $0.5 \mathrm{~Hz}, 1 \mathrm{H}), 1.90$ (s, 3H), 1.42 (s, 9H).

${ }^{13}$ C NMR (125 MHz, $\mathrm{CDCl}_{3}$ ) $\delta 151.50$ (e), 148.76 (o), 147.34 (o), 140.26 (o), 139.30 (e), 133.02 (o), 123.14 (o), 116.15 (e), 82.44 (e), 82.40 (e), 27.90 (o), 25.78 (o). 
IR (Neat) 3089 (w), 2980 (w), 2935 (w), 1740 (s), 1641 (w), 1417 (m), 1369 (m), 1277 (vs), 1153 (s), 1095 (s), 1021 (m), 927 (m), 847 (s), 792 (m), 713 (s) cm $\mathrm{cm}^{-1}$.

HRMS (EI, $\mathrm{M}^{+}$) calcd for $\mathrm{C}_{14} \mathrm{H}_{19} \mathrm{NO}_{3} 249.1365$, found 249.1373 .

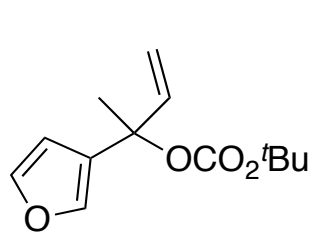

tert-Butyl (2-(furan-3-yl)but-3-en-2-yl) carbonate (2z)

Color and State: Pale yellow oil. (Method A)

${ }^{1} \mathbf{H}$ NMR $\left(500 \mathrm{MHz}, \mathrm{CDCl}_{3}\right) \delta$ 7.39-7.36 (m, 2H), 6.41-6.40 (m, 1H), 6.28 $(\mathrm{dd}, J=17.5,10.8 \mathrm{~Hz}, 1 \mathrm{H}), 5.23(\mathrm{dd}, J=17.4,0.3 \mathrm{~Hz}, 1 \mathrm{H}), 5.22$ (dd, $J=$ $10.8,0.5 \mathrm{~Hz}, 1 \mathrm{H}), 1.84$ (s, 3H), 1.44 (s, 9H).

${ }^{13}$ C NMR (125 MHz, $\mathrm{CDCl}_{3}$ ) $\delta 151.67$ (e), 143.19 (o), 140.83 (o), 139.75 (o), 128.89 (e), 114.70 (e), 109.24 (o), 81.90 (e), 79.75 (e), 27.95 (o), 25.72 (o).

IR (Neat) 3090 (w), 2979 (w), 2934 (w), 2873 (w), 1740 (s), 1643 (w), 1368 (m), 1273 (s), 1252 (s), 1156 (s), 1119 (s), 1062 (s), 1021 (m), 923 (m), 873 (m), 833 (m), 791 (s), 730 (m), 668 (w) $\mathrm{cm}^{-1}$.

HRMS (EI, $\mathrm{M}^{+}$) calcd for $\mathrm{C}_{13} \mathrm{H}_{18} \mathrm{O}_{4} 238.1205$, found 238.1200.

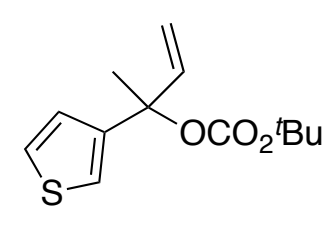

tert-Butyl (2-(thiophen-3-yl)but-3-en-2-yl) carbonate (2aa)

Color and State: Colorless oil. (Method A)

${ }^{1} \mathbf{H}$ NMR $\left(500 \mathrm{MHz}, \mathrm{CDCl}_{3}\right) \delta 7.27(\mathrm{dd}, J=5.4,3.3 \mathrm{~Hz}, 1 \mathrm{H}), 7.19$ (dd, $J=$ 3.0, $1.2 \mathrm{~Hz}, 1 \mathrm{H}), 7.06(\mathrm{dd}, J=5.0,1.2 \mathrm{~Hz}, 1 \mathrm{H}), 6.32(\mathrm{dd}, J=17.5,10.7 \mathrm{~Hz}$, 1H), 5.23 (d, $J=16.9 \mathrm{~Hz}, 1 \mathrm{H}), 5.23$ (d, $J=11.3 \mathrm{~Hz}, 1 \mathrm{H}), 1.90$ (s, 3H), 1.42 (s, 9H).

${ }^{13}$ C NMR (125 MHz, $\mathrm{CDCl}_{3}$ ) $\delta 151.61$ (e), 144.94 (e), 141.09 (o), 126.06 (o), 125.80 (o), 121.09 (o), 114.69 (e), 82.07 (e), 81.87 (e), 27.90 (o), 25.76 (o).

IR (Neat) 3107 (w), 2979 (w), 2935 (w), 1740 (s), 1641 (w), 1367 (m), 1275 (s), 1149 (vs), 1089 (s), $924(\mathrm{~m}), 839$ (s), $784(\mathrm{~s}), 692(\mathrm{w}) \mathrm{cm}^{-1}$.

HRMS (EI, $\mathrm{M}^{+}$) calcd for $\mathrm{C}_{13} \mathrm{H}_{18} \mathrm{O}_{3} \mathrm{~S} 254.0977$, found 254.0968 .

\section{Representative Experimental Procedure for the Regioselective Rhodium-Catalyzed} Allylic Cyanomethylation 3a-aa

From the Allylic Carbonate: $\mathrm{Rh}(\mathrm{COD})_{2} \mathrm{OTf}(23.0 \mathrm{mg}, 0.05 \mathrm{mmol})$ and triphenyl phosphite $(39.0$ $\mu \mathrm{L}, 0.15 \mathrm{mmol})$ were dissolved in tetrahydrofuran $(3 \mathrm{~mL})$ at room temperature. The mixture was 
stirred for ca. 5 minutes, resulting in a light yellow homogeneous solution, then 2-(tertbutyldimethylsilyl)acetonitrile (1d) $(0.155 \mathrm{~g}, 1.0 \mathrm{mmol})$ was added dropwise as a solution in tetrahydrofuran $(2 \mathrm{~mL})$. The reaction mixture was cooled to $0{ }^{\circ} \mathrm{C}$ and lithium bis(trimethylsilyl)amide $(0.95 \mathrm{~mL}, 0.95 \mathrm{mmol} ; 1 \mathrm{M}$ solution in tetrahydrofuran) was added dropwise, followed immediately by the addition of methyl (3-methyl-5-phenylpent-1-en-3-yl) carbonate (2a) (117.0 mg, $0.50 \mathrm{mmol})$ via tared $500 \mu \mathrm{L}$ gastight syringe. The mixture was allowed to slowly warm to room temperature and stirred for $c a$. 16 hours. Tetrabutylammonium fluoride (1.5 mL, $1.5 \mathrm{mmol} ; 1 \mathrm{M}$ solution in tetrahydrofuran) was then added dropwise and the solution was allowed to stir at room temperature for $c a$. 2 hours (TLC control). The reaction mixture was quenched with sat. aq $\mathrm{NH}_{4} \mathrm{Cl}$ and extracted with diethyl ether. The organic phases were combined, dried (anhyd. $\mathrm{MgSO}_{4}$ ), filtered and concentrated in vacuo to afford the crude product. Purification by flash column chromatography (silica gel, eluting with $2-10 \%$ diethyl ether/hexane) afforded the $\beta$-quaternary nitrile $\mathbf{3 a}(87.6 \mathrm{mg}, 88 \%)$ as a colorless oil.

From the Allylic Alcohol: $n$-Butyllithium $(0.21 \mathrm{~mL}, 0.53 \mathrm{mmol} ; 2.5 \mathrm{M}$ in hexanes) was added dropwise to a stirring solution of 2-(4-methoxypenyl)but-3-en-2-ol (2p') (89.0 mg, $0.5 \mathrm{mmol})$ in tetrahydrofuran $(1.5 \mathrm{~mL})$ at $-78^{\circ} \mathrm{C}$. The anion was allowed to form over $c a .30$ minutes, then ditert-butyl dicarbonate $(115.0 \mathrm{mg}, 0.53 \mathrm{mmol})$ in tetrahydrofuran $(0.5 \mathrm{~mL}, 26.0 \mathrm{mmol})$ was added dropwise and the reaction was allowed to slowly warm to $0{ }^{\circ} \mathrm{C}$ and stirred for $c a$. 16 hours. In a separate flask, $\operatorname{Rh}(\mathrm{COD})_{2} \mathrm{OTf}(23.0 \mathrm{mg}, 0.05 \mathrm{mmol})$ and triphenyl phosphite $(39.0 \mu \mathrm{L}, 0.15 \mathrm{mmol})$ were dissolved in tetrahydrofuran $(2 \mathrm{~mL})$ at room temperature. The mixture was stirred for $c a .5$ minutes, resulting in a light yellow homogeneous solution, then 2-(tertbutyldimethylsilyl)acetonitrile (1d) (0.155 g, $1.0 \mathrm{mmol})$ was added dropwise as a solution in tetrahydrofuran $(1 \mathrm{~mL})$. The reaction mixture was cooled to $0{ }^{\circ} \mathrm{C}$ and lithium bis(trimethylsilyl)amide $(0.95 \mathrm{~mL}, 0.95 \mathrm{mmol} ; 1 \mathrm{M}$ solution in tetrahydrofuran) was added dropwise, followed by the immediate addition of the allylic carbonate solution via Teflon ${ }^{\circledR}$ cannula, rinsing the flask with tetrahydrofuran $(0.5 \mathrm{~mL})$. The resulting mixture was then allowed to slowly warm to room temperature and stirred for $c a$. 16 hours, before tetrabutylammonium fluoride $(1.5 \mathrm{~mL}, 1.5 \mathrm{mmol} ; 1 \mathrm{M}$ solution in tetrahydrofuran) was added dropwise at room temperature and the solution stirred for an additional ca. 2 hours (TLC control). The reaction 
mixture was quenched with sat. aq $\mathrm{NH}_{4} \mathrm{Cl}$ and extracted with diethyl ether. The organic phases were combined, dried (anhyd. $\mathrm{MgSO}_{4}$ ), filtered and concentrated in vacuo to afford the crude product. Purification by flash column chromatography (silica gel, eluting with $2-10 \%$ diethyl ether/hexane) afforded the $\beta$-quaternary nitrile $3 \mathbf{p}(69.7 \mathrm{mg}, 70 \%)$ as a colorless oil.

\section{Spectral Data for the $\beta$-Quaternary Nitrile Products 3a-aa}

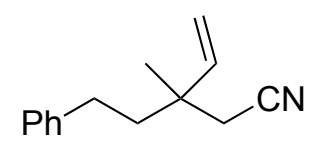

\section{3-Methyl-3-phenethylpent-4-enenitrile (3a)}

Color and State: Yellow oil; $b: l \geq 19: 1$

${ }^{1} \mathbf{H}$ NMR $\left(500 \mathrm{MHz}, \mathrm{CDCl}_{3}\right) \delta$ 7.30-7.27 (m, 2H), 7.21-7.16 (m, 3H), 5.83

(dd, $J=17.5,10.8 \mathrm{~Hz}, 1 \mathrm{H}), 5.23$ (d, $J=10.9 \mathrm{~Hz}, 1 \mathrm{H}), 5.13$ (d, $J=17.5 \mathrm{~Hz}, 1 \mathrm{H}), 2.59-2.50$ (m, $2 \mathrm{H}), 2.40(\mathrm{~s}, 2 \mathrm{H}), 1.84-1.74(\mathrm{~m}, 2 \mathrm{H}), 1.26(\mathrm{~s}, 3 \mathrm{H})$.

${ }^{13}$ C NMR (125 MHz, CDCl $) \delta 142.95$ (o), 141.79 (e), 128.61 (o), 128.38 (o), 126.13 (o), 117.96 (e), 114.59 (e), 42.22 (e), 39.05 (e), 30.79 (e), 29.31 (e), 23.49 (o).

IR (Neat) 3084 (w), 3026 (w), 2965 (w), 2930 (w), 2862 (w), 2246 (w), 1640 (w), 1602 (w), 1495 (w), 1454 (m), 1415 (w), 1379 (w), 1000 (w), 919 (m), 733 (m), 698 (vs) cm-1.

HRMS (ESI, $[\mathrm{M}+\mathrm{H}]^{+}$) calcd for $\mathrm{C}_{14} \mathrm{H}_{18} \mathrm{~N} 200.1434$, found 200.1441 .

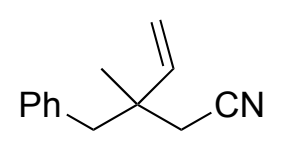

\section{3-Benzyl-3-methylpent-4-enenitrile (3b)}

Color and State: Yellow oil; $b: l \geq 19: 1$

${ }^{1} \mathbf{H}$ NMR $\left(500 \mathrm{MHz}, \mathrm{CDCl}_{3}\right) \delta$ 7.31-7.23 (m, 3H), 7.17-7.15 (m, 2H), $5.86(\mathrm{dd}$, $J=17.4,10.8 \mathrm{~Hz}, 1 \mathrm{H}), 5.15$ (d, $J=10.8 \mathrm{~Hz}, 1 \mathrm{H}), 5.00$ (d, $J=17.4 \mathrm{~Hz}, 1 \mathrm{H}), 2.77$ (s, 2H), 2.29 (s, $2 \mathrm{H}), 1.19(\mathrm{~s}, 3 \mathrm{H})$.

${ }^{13}$ C NMR (125 MHz, $\mathrm{CDCl}_{3}$ ) $\delta 143.11$ (o), 136.58 (e), 130.63 (o), 128.26 (o), 126.93 (o), 118.33 (e), 114.20 (e), 46.22 (e), 39.91 (e), 28.11 (e), 23.99 (o).

IR (Neat) 3085 (w), 3028 (w), 2967 (w), 2924 (w), 2854 (w), 2245 (w), 1640 (w), 1602 (w), 1494 (w), 1453 (m), 1415 (w), 1378 (w), 1000 (w), 919 (m), 764 (m), 702 (vs) cm ${ }^{-1}$.

HRMS (EI, $\mathrm{M}^{+}$) calcd for $\mathrm{C}_{13} \mathrm{H}_{15} \mathrm{~N}$ 185.1204, found 185.1200. 


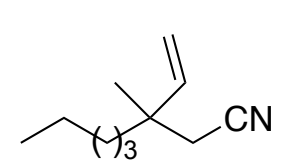

\section{3-Methyl-3-vinyloctanenitrile (3c)}

Color and State: Colorless oil; $b: l \geq 19: 1$

${ }^{1} \mathbf{H}$ NMR $\left(500 \mathrm{MHz}, \mathrm{CDCl}_{3}\right) \delta 5.75(\mathrm{dd}, J=17.5,10.8 \mathrm{~Hz}, 1 \mathrm{H}), 5.13(\mathrm{~d}, J=$

$10.9 \mathrm{~Hz}, 1 \mathrm{H}), 5.04(\mathrm{~d}, J=17.6 \mathrm{~Hz}, 1 \mathrm{H}), 2.33(\mathrm{~s}, 2 \mathrm{H}), 1.49-1.40(\mathrm{~m}, 2 \mathrm{H}), 1.35-1.18(\mathrm{~m}, 6 \mathrm{H}), 1.16$ (s, 3H), $0.88(\mathrm{t}, J=7.1 \mathrm{~Hz}, 3 \mathrm{H})$.

${ }^{13}$ C NMR (125 MHz, $\mathrm{CDCl}_{3}$ ) $\delta 143.56$ (o), 118.24 (e), 113.88 (e), 40.20 (e), 38.93 (e), 32.34 (e), 29.21 (e), 23.89 (e), 23.63 (o), 22.66 (e), 14.14 (o).

IR (Neat) 3085 (w), 2957 (s), 2929 (s), 2860 (m), 2245 (w), 1742 (w), 1640 (w), 1463 (m), 1416 (m), $1378(\mathrm{~m}), 1260(\mathrm{w}), 1000(\mathrm{~m}), 918(\mathrm{~s}), 727(\mathrm{w}), 682(\mathrm{w}) \mathrm{cm}^{-1}$.

HRMS (ESI, $[\mathrm{M}+\mathrm{H}]^{+}$) calcd for $\mathrm{C}_{11} \mathrm{H}_{20} \mathrm{~N}$ 166.1590, found 166.1597 .

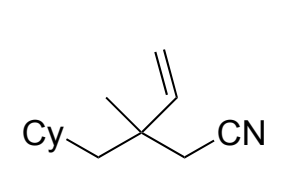

\section{3-(Cyclohexymethyl)-3-methylpent-4-enenitrile (3d)}

Color and State: Colorless oil; $b: l \geq 19: 1$

${ }^{1} \mathbf{H}$ NMR $\left(500 \mathrm{MHz}, \mathrm{CDCl}_{3}\right) \delta 5.78(\mathrm{dd}, J=17.5,10.8 \mathrm{~Hz}, 1 \mathrm{H}), 5.11(\mathrm{~d}, J=$ $10.8 \mathrm{~Hz}, 1 \mathrm{H}), 5.04$ (d, $J=17.5 \mathrm{~Hz}, 1 \mathrm{H}), 2.32$ (s, 2H), 1.69-1.58 (m, 5H), 1.39 (dd, A of ABX, $\left.J_{\mathrm{AB}}=14.0 \mathrm{~Hz}, J_{\mathrm{AX}}=5.5 \mathrm{~Hz}, 1 \mathrm{H}\right), 1.33\left(\mathrm{dd}, \mathrm{B}\right.$ of $\left.\mathrm{ABX}, J_{\mathrm{AB}}=14.1 \mathrm{~Hz}, J_{\mathrm{BX}}=4.9 \mathrm{~Hz}, 1 \mathrm{H}\right), 1.29-$ $1.16(\mathrm{~m}, 3 \mathrm{H}), 1.18(\mathrm{~s}, 3 \mathrm{H}), 1.15-1.07$ (m, 1H), 1.00-0.90 (m, 2H).

${ }^{13}$ C NMR (125 MHz, $\left.\mathrm{CDCl}_{3}\right) \delta 144.03$ (o), 118.27 (e), 113.60 (e), 47.91 (e), 39.38 (e), 35.56 (e), 35.35 (e), 34.06 (o), 30.04 (e), 26.50 (e), 26.47 (e), 26.22 (e), 23.96 (o).

IR (Neat) 3085 (w), 2919 (vs), 2849 (m), 2246 (w), 1640 (w), 1447 (m), 1415 (m), 1378 (w) 1261 (w), $1000(\mathrm{~m}), 916(\mathrm{~s}), 843(\mathrm{w}), 683(\mathrm{w}) \mathrm{cm}^{-1}$.

HRMS (ESI, $[\mathrm{M}+\mathrm{H}]^{+}$) calcd for $\mathrm{C}_{13} \mathrm{H}_{22} \mathrm{~N}$ 192.1747, found 192.1743 .

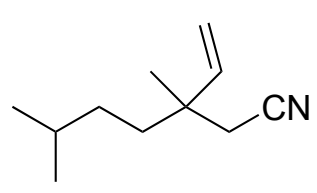

\section{3,6-Dimethyl-3-vinylheptanenitrile (3e)}

Color and State: Colorless oil; $b: l \geq 19: 1$

${ }^{1}$ H NMR $\left(500 \mathrm{MHz}, \mathrm{CDCl}_{3}\right) \delta 5.74(\mathrm{dd}, J=17.5,10.8 \mathrm{~Hz}, 1 \mathrm{H}), 5.13(\mathrm{dd}, J$ $=10.8,0.5 \mathrm{~Hz}, 1 \mathrm{H}), 5.04(\mathrm{dd}, J=17.4,0.5 \mathrm{~Hz}, 1 \mathrm{H}), 2.33(\mathrm{~s}, 2 \mathrm{H}), 1.53-1.40(\mathrm{~m}, 3 \mathrm{H}), 1.16(\mathrm{~s}, 3 \mathrm{H})$, $1.13-1.05(\mathrm{~m}, 2 \mathrm{H}), 0.88(\mathrm{~d}, J=6.6 \mathrm{~Hz}, 6 \mathrm{H})$.

${ }^{13}$ C NMR (125 MHz, CDCl $) \delta 143.55$ (o), 118.24 (e), 113.95 (e), 38.84 (e), 37.99 (e), 33.19 (e), 29.22 (e), 28.57 (o), 23.63 (o), 22.71 (o), 22.69 (o). 
IR (Neat) 3085 (w), 2956 (s) 2929 (s), 2870 (m), 2245 (w), 1641 (w), 1466 (m), 1416 (m), 1381 (m), $1168(\mathrm{w}), 1000(\mathrm{~m}), 917(\mathrm{~s}), 762(\mathrm{w}), 682(\mathrm{w}) \mathrm{cm}^{-1}$.

HRMS (ESI, $[\mathrm{M}+\mathrm{H}]^{+}$) calcd for $\mathrm{C}_{11} \mathrm{H}_{20} \mathrm{~N}$ 166.1590, found 166.1587 .

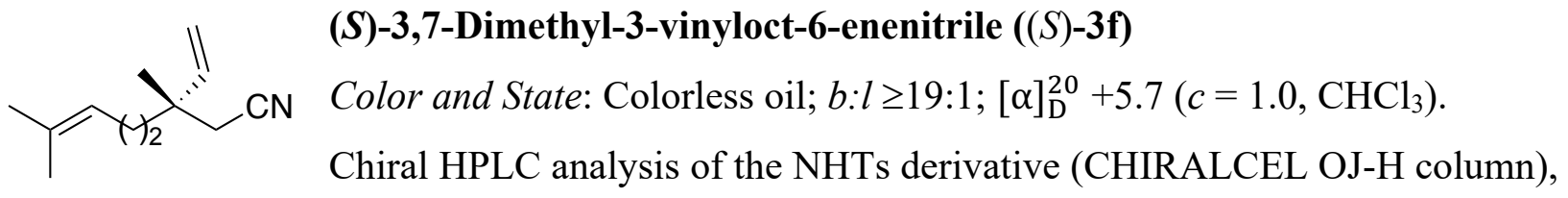

99:1 hexane/isopropanol at $0.7 \mathrm{~mL} / \mathrm{min}$. flow rate; $t_{\mathrm{R}}(S)$-enantiomer (major) $=32.4 \mathrm{~min}$., $t_{\mathrm{R}}(R)$ enantiomer $($ minor $)=36.3 \mathrm{~min} . ; 94 \%$ ee.

${ }^{1}$ H NMR $\left(500 \mathrm{MHz}, \mathrm{CDCl}_{3}\right) \delta 5.76(\mathrm{dd}, J=17.5,10.8 \mathrm{~Hz}, 1 \mathrm{H}), 5.15(\mathrm{dd}, J=10.8,0.5 \mathrm{~Hz}, 1 \mathrm{H})$, 5.09-5.05 (m, 1H), $5.06(\mathrm{dd}, J=17.5,0.4 \mathrm{~Hz}, 1 \mathrm{H}), 2.35(\mathrm{~s}, 2 \mathrm{H}), 1.92\left(\mathrm{dt}, \mathrm{A}\right.$ of $\mathrm{ABX}_{2}, J_{\mathrm{AB}}=15.7$ $\left.\mathrm{Hz}, J_{\mathrm{AX}}=7.3 \mathrm{~Hz}, 1 \mathrm{H}\right), 1.89\left(\mathrm{dt}, \mathrm{B}\right.$ of $\left.\mathrm{ABX}_{2}, J_{\mathrm{AB}}=15.7 \mathrm{~Hz}, J_{\mathrm{BX}}=7.3 \mathrm{~Hz}, 1 \mathrm{H}\right), 1.68(\mathrm{~s}, 3 \mathrm{H}), 1.59$ (s, 3H), 1.54-1.43 (m, 2H), $1.18(\mathrm{~s}, 3 \mathrm{H})$.

${ }^{13}$ C NMR (125 MHz, $\mathrm{CDCl}_{3}$ ) $\delta 143.22$ (o), 132.16 (e), 123.71 (o), 118.09 (e), 114.07 (e), 40.12 (e), 38.90 (e), 29.16 (e), 25.74 (o), 23.47 (o), 22.96 (e), 17.70 (o).

IR (Neat) 3085 (w), 2967 (m), 2918 (m), 2856 (m), 2245 (w), 1640 (w), 1453 (m), 1416 (m), 1378 (m), 1137 (w), $1100(\mathrm{w}), 999(\mathrm{~m}), 918$ (vs) $831(\mathrm{~m}), 747(\mathrm{w}), 682(\mathrm{w}) \mathrm{cm}^{-1}$.

HRMS (ESI, $[\mathrm{M}+\mathrm{H}]^{+}$) calcd for $\mathrm{C}_{12} \mathrm{H}_{20} \mathrm{~N}$ 178.1590, found 178.1595 .

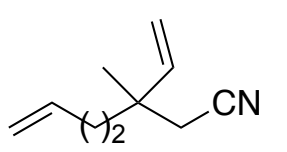

\section{3-Methyl-3-vinylhept-6-enenitrile (3g)}

Color and State: Colorless oil; $b: l \geq 19: 1$

${ }^{1} \mathbf{H}$ NMR $\left(500 \mathrm{MHz}, \mathrm{CDCl}_{3}\right) \delta 5.79(\mathrm{ddt}, J=17.1,10.5,6.7 \mathrm{~Hz}, 1 \mathrm{H}), 5.75(\mathrm{dd}$, $J=17.5,10.8 \mathrm{~Hz}, 1 \mathrm{H}), 5.16(\mathrm{dd}, J=10.8,0.6 \mathrm{~Hz}, 1 \mathrm{H}), 5.07$ (dd, $J=17.5,0.5 \mathrm{~Hz}, 1 \mathrm{H}), 5.02$ (app. $\mathrm{dq}, J=17.1,1.7 \mathrm{~Hz}, 1 \mathrm{H}), 4.98-4.95(\mathrm{~m}, 1 \mathrm{H}), 2.35$ (s, 2H), 2.04-1.96 (m, 2H), 1.61-1.51 (m, 2H), $1.19(\mathrm{~s}, 3 \mathrm{H})$.

${ }^{13}$ C NMR (125 MHz, $\left.\mathrm{CDCl}_{3}\right) \delta 143.06$ (o), 138.11 (o), 118.03 (e), 115.03 (e), 114.37 (e), 39.31 (e), 38.90 (e), 29.36 (e), 28.65 (e), 23.47 (o).

IR (Neat) 3080 (w), 2972 (w), 2923 (w), 2853 (w), 2245 (w), 1640 (m), 1456 (w), 1416 (m), 1379 (w), 1138 (w), 998 (m), 912 (vs), $772(\mathrm{w}), 684(\mathrm{w}) \mathrm{cm}^{-1}$.

HRMS (ESI, $[\mathrm{M}+\mathrm{H}]^{+}$) calcd for $\mathrm{C}_{10} \mathrm{H}_{16} \mathrm{~N}$ 150.1277, found 150.1274 . 


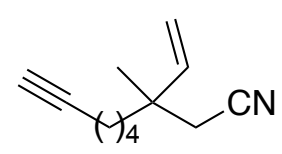

\section{3-Methyl-3-vinylnon-8-ynenitrile (3h)}

Color and State: Colorless oil; $b: l \geq 19: 1$

${ }^{1}$ H NMR $\left(500 \mathrm{MHz}, \mathrm{CDCl}_{3}\right) \delta 5.75(\mathrm{dd}, J=17.6,10.8 \mathrm{~Hz}, 1 \mathrm{H}), 5.15(\mathrm{~d}, J=$ $10.8 \mathrm{~Hz}, 1 \mathrm{H}), 5.06$ (d, $J=17.4 \mathrm{~Hz}, 1 \mathrm{H}), 2.34$ (s, 2H), $2.20(\mathrm{td}, J=7.0,2.7 \mathrm{~Hz}, 2 \mathrm{H}), 1.94$ (t, $J=$ $2.6 \mathrm{~Hz}, 1 \mathrm{H}), 1.55-1.45(\mathrm{~m}, 4 \mathrm{H}), 1.41-1.30(\mathrm{~m}, 2 \mathrm{H}), 1.18(\mathrm{~s}, 3 \mathrm{H})$.

${ }^{13}$ C NMR (125 MHz, $\mathrm{CDCl}_{3}$ ) $\delta 143.25$ (o), 118.10 (e), 114.19 (e), 84.24 (e), 68.66 (e), 39.63 (e), 38.92 (e), 29.28 (e), 28.81 (e), 23.56 (o), 23.35 (e), 18.40 (e).

IR (Neat) 3295 (w), 3085 (w), 2939 (m), 2864 (w), 2245 (w), 2116 (w), 1640 (w), 1463 (m), 1416 (m), 1379 (w), 1138 (w), 1000 (m), 920 (s), 652 (vs) cm ${ }^{-1}$.

HRMS (ESI, $[\mathrm{M}+\mathrm{Na}]^{+}$) calcd for $\mathrm{C}_{12} \mathrm{H}_{17} \mathrm{NNa}$ 198.1253, found, 198.1257.

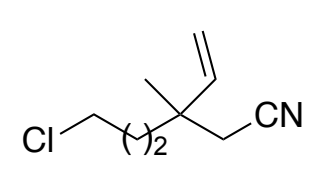

\section{6-Chloro-3-methyl-3-vinylhexanenitrile (3i)}

Color and State: Pale yellow oil; $b: l \geq 19: 1$

${ }^{1}$ H NMR $\left(500 \mathrm{MHz}, \mathrm{CDCl}_{3}\right) \delta 5.74(\mathrm{dd}, J=17.6,10.8 \mathrm{~Hz}, 1 \mathrm{H}), 5.18(\mathrm{~d}, J=$ $10.8 \mathrm{~Hz}, 1 \mathrm{H}), 5.08$ (d, $J=17.4 \mathrm{~Hz}, 1 \mathrm{H}), 3.52$ (t, $J=6.4 \mathrm{~Hz}, 2 \mathrm{H}), 2.35$ (s, 2H), 1.78-1.58 (m, 4H), $1.19(\mathrm{~s}, 3 \mathrm{H})$.

${ }^{13}$ C NMR (125 MHz, $\left.\mathrm{CDCl}_{3}\right) \delta 142.64$ (o), 117.83 (e), 114.82 (e), 45.09 (e), 38.68 (e), 37.36 (e), 29.55 (e), 27.68 (e), 23.34 (o).

IR (Neat) 3085 (w), 2962 (m), 2926 (w), 2873 (w), 2246 (w), 1640 (w), 1460 (m), 1416 (m), 1380 (w), 1306 (m), 1139 (w), 1000 (m), 922 (vs), 773 (m), 685 (m) cm-1.

HRMS (ESI, $[\mathrm{M}+\mathrm{H}]^{+}$) calcd for $\mathrm{C}_{9} \mathrm{H}_{15}{ }^{35} \mathrm{ClN} 172.0888$, found 172.0889 .

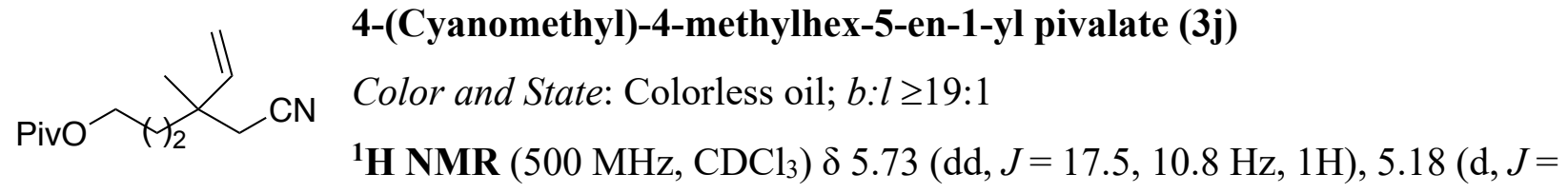
$10.8 \mathrm{~Hz}, 1 \mathrm{H}), 5.08$ (d, $J=17.5 \mathrm{~Hz}, 1 \mathrm{H}), 4.04$ (t, $J=6.0 \mathrm{~Hz}, 2 \mathrm{H}), 2.35$ (s, 2H), 1.61-1.49 (m, 4H), $1.20(\mathrm{~s}, 9 \mathrm{H}), 1.19(\mathrm{~s}, 3 \mathrm{H})$.

${ }^{13}$ C NMR (125 MHz, CDCl $) \delta 178.60$ (e), 142.76 (o), 117.84 (e), 114.62 (e), 64.22 (e), 38.87 (e), 38.64 (e), 36.27 (e), 29.38 (e), 27.32 (o), 23.77 (e), 23.39 (o).

IR (Neat) 3086 (w), 2965 (w), 2937 (w), 2873 (w), 2246 (w), 1722 (s), 1640 (w), 1479 (w), 1460 (w), 1365 (w), 1283 (m), 1149 (vs), $1036(\mathrm{w}), 1000$ (w), 921 (m), 771 (w), 683 (w) cm ${ }^{-1}$. 
HRMS (ESI, $[\mathrm{M}+\mathrm{H}]^{+}$) calcd for $\mathrm{C}_{14} \mathrm{H}_{24} \mathrm{NO}_{2} 238.1802$, found 238.1806 .

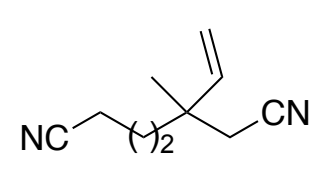

\section{3-Methyl-3-vinylheptanedinitrile (3k)}

Color and State: Yellow oil; $b: l \geq 19: 1$

${ }^{1} \mathrm{H}$ NMR $\left(500 \mathrm{MHz}, \mathrm{CDCl}_{3}\right) \delta 5.73(\mathrm{dd}, J=17.4,10.8 \mathrm{~Hz}, 1 \mathrm{H}), 5.21(\mathrm{~d}, J=$ $10.8 \mathrm{~Hz}, 1 \mathrm{H}), 5.10$ (d, $J=17.4 \mathrm{~Hz}, 1 \mathrm{H}), 2.37-2.34$ (m, 4H), 1.64-1.59 (m, 4H), 1.20 (s, 3H).

${ }^{13}$ C NMR (125 MHz, $\left.\mathrm{CDCl}_{3}\right) \delta 142.18$ (o), 119.26 (e), 117.58 (e), 115.20 (e), 38.99 (e), 38.74 (e) 29.54 (e), 23.16 (o), 20.68 (e), 17.61 (e).

IR (Neat) 3086 (w), 2966 (m), 2921 (w), 2876 (w), 2245 (w), 1748 (w), 1640 (w), 1460 (m), 1418 (m), $1380(\mathrm{w}), 1140(\mathrm{w}), 1002(\mathrm{~m}), 924$ (vs), 737 (w), 685 (w) $\mathrm{cm}^{-1}$.

HRMS (ESI, $[\mathrm{M}+\mathrm{H}]^{+}$) calcd for $\mathrm{C}_{10} \mathrm{H}_{15} \mathrm{~N}_{2}$ 163.1230, found 126.1229 .

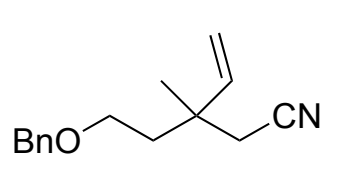

\section{3-(2-(Benzyloxy)ethyl)-3-methylpent-4-enenitrile (3I)}

Color and State: Colorless oil; $b: l \geq 19: 1$

${ }^{1} \mathbf{H}$ NMR $\left(500 \mathrm{MHz}, \mathrm{CDCl}_{3}\right) \delta$ 7.37-7.27 (m, 5H), $5.79(\mathrm{dd}, J=17.5,10.9$

Hz, 1H), 5.14 (d, $J=10.8 \mathrm{~Hz}, 1 \mathrm{H}), 5.06$ (d, $J=17.5 \mathrm{~Hz}, 1 \mathrm{H}), 4.47$ (s, 2H), 3.51 (t, $J=6.4 \mathrm{~Hz}$, $2 \mathrm{H}), 2.47\left(\mathrm{~d}, \mathrm{~A}\right.$ of $\left.\mathrm{AB}, J_{\mathrm{AB}}=16.5 \mathrm{~Hz}, 1 \mathrm{H}\right), 2.43\left(\mathrm{~d}, \mathrm{~B}\right.$ of $\left.\mathrm{AB}, J_{\mathrm{AB}}=16.6 \mathrm{~Hz}, 1 \mathrm{H}\right), 1.84(\mathrm{dt}, \mathrm{A}$ of $\left.\mathrm{ABX}_{2}, J_{\mathrm{AB}}=14.2 \mathrm{~Hz}, J_{\mathrm{AX}}=6.6 \mathrm{~Hz}, 1 \mathrm{H}\right), 1.79\left(\mathrm{dt}, \mathrm{B}\right.$ of $\left.\mathrm{ABX} 2, J_{\mathrm{AB}}=14.4 \mathrm{~Hz}, J_{\mathrm{BX}}=6.5 \mathrm{~Hz}, 1 \mathrm{H}\right)$, $1.22(\mathrm{~s}, 3 \mathrm{H})$.

${ }^{13}$ C NMR (125 MHz, $\mathrm{CDCl}_{3}$ ) $\delta 143.11$ (o), 138.31 (e), 128.56 (o), 127.78 (o), 127.72 (o), 118.28 (e), 114.15 (e), 73.24 (e), 66.84 (e), 39.38 (e), 38.26 (e), 29.39 (e), 24.32 (o).

IR (Neat) 3085 (w), 3030 (w), 2965 (w), 2930 (w), 2866 (w), 2243 (w), 1639 (w), 1453 (m), 1415 (w), 1364 (m), 1097 (s), 999 (m), 919 (m), 735 (s), 697 (vs) $\mathrm{cm}^{-1}$.

HRMS (ESI, $[\mathrm{M}+\mathrm{H}]^{+}$) calcd for $\mathrm{C}_{15} \mathrm{H}_{20} \mathrm{NO} 230.1539$, found 230.1536 .

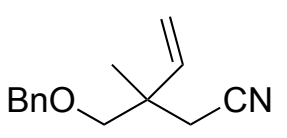

\section{3-((Benzyloxy)methyl)-3-methylpent-4-enenitrile (3m)}

Color and State: Colorless oil; $b: l \geq 19: 1$

${ }^{1} \mathbf{H}$ NMR $\left(500 \mathrm{MHz}, \mathrm{CDCl}_{3}\right) \delta$ 7.37-7.28 (m, 5H), $5.86(\mathrm{dd}, J=17.6,11.0 \mathrm{~Hz}$, $1 \mathrm{H}), 5.19(\mathrm{~d}, J=11.4 \mathrm{~Hz}, 1 \mathrm{H}), 5.16(\mathrm{~d}, J=17.7 \mathrm{~Hz}, 1 \mathrm{H}), 4.54(\mathrm{~s}, 2 \mathrm{H}), 3.37\left(\mathrm{~d}, \mathrm{~A}\right.$ of AB, $J_{\mathrm{AB}}=9.1$ $\mathrm{Hz}, 1 \mathrm{H}), 3.33\left(\mathrm{~d}, \mathrm{~B}\right.$ of $\left.\mathrm{AB}, J_{\mathrm{AB}}=9.2 \mathrm{~Hz}, 1 \mathrm{H}\right), 2.54\left(\mathrm{~d}, \mathrm{~A}\right.$ of $\left.\mathrm{AB}, J_{\mathrm{AB}}=16.6 \mathrm{~Hz}, 1 \mathrm{H}\right), 2.49(\mathrm{~d}, \mathrm{~B}$ of $\left.\mathrm{AB}, J_{\mathrm{AB}}=16.4 \mathrm{~Hz}, 1 \mathrm{H}\right), 1.20(\mathrm{~s}, 3 \mathrm{H})$. 
${ }^{13}$ C NMR (125 MHz, $\mathrm{CDCl}_{3}$ ) $\delta 140.92$ (o), 138.09 (e), 128.54 (o), 127.86 (o), 127.66 (o), 118.18 (e), 115.06 (e), 75.91 (e), 73.56 (e), 40.27 (e), 26.08 (e), 21.52 (o).

IR (Neat) 3086 (w), 3030 (w), 2968 (w), 2931 (w), 2859 (w), 2244 (w), 1642 (w), 1453 (m), 1416 (w), 1360 (w), 1094 (s), 998 (m), 923 (m), 736 (s), 697 (vs) cm $\mathrm{cm}^{-1}$.

HRMS (EI, $\mathrm{M}^{+}$) calcd for $\mathrm{C}_{14} \mathrm{H}_{17} \mathrm{NO} 215.1310$, found 215.1317 .

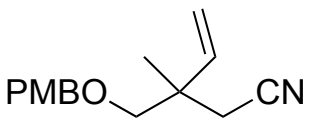

\section{3-(((4-Methoxybenzyl)oxy)methyl)-3-methylpent-4-enenitrile (3n)}

Color and State: Colorless oil; $b: l \geq 19: 1$

${ }^{1} \mathbf{H}$ NMR $\left(500 \mathrm{MHz}, \mathrm{CDCl}_{3}\right) \delta$ 7.25-7.22 (m, 2H), 6.90-6.87 (m, 2H), 5.85

$(\mathrm{dd}, J=17.5,10.9 \mathrm{~Hz}, 1 \mathrm{H}), 5.18(\mathrm{dd}, J=10.9,0.5 \mathrm{~Hz}, 1 \mathrm{H}), 5.15(\mathrm{dd}, J=18.1,0.6 \mathrm{~Hz}, 1 \mathrm{H}), 4.46$ $(\mathrm{s}, 2 \mathrm{H}), 3.81(\mathrm{~s}, 3 \mathrm{H}), 3.33\left(\mathrm{~d}, \mathrm{~A}\right.$ of $\left.\mathrm{AB}, J_{\mathrm{AB}}=9.1 \mathrm{~Hz}, 1 \mathrm{H}\right), 3.29\left(\mathrm{~d}, \mathrm{~B}\right.$ of $\left.\mathrm{AB}, J_{\mathrm{AB}}=9.2 \mathrm{~Hz}, 1 \mathrm{H}\right)$, $2.51\left(\mathrm{~d}, \mathrm{~A}\right.$ of $\left.\mathrm{AB}, J_{\mathrm{AB}}=16.6 \mathrm{~Hz}, 1 \mathrm{H}\right), 2.47\left(\mathrm{~d}, \mathrm{~B}\right.$ of $\left.\mathrm{AB}, J_{\mathrm{AB}}=16.6 \mathrm{~Hz}, 1 \mathrm{H}\right), 1.18(\mathrm{~s}, 3 \mathrm{H})$.

${ }^{13}$ C NMR (125 MHz, $\mathrm{CDCl}_{3}$ ) $\delta 159.40$ (e), 140.99 (o), 130.17 (e), 129.32 (o), 118.25 (e), 115.03 (e), 113.94 (o), 75.63 (e), 73.26 (e), 55.41 (o), 40.25 (e), 26.10 (e), 21.55 (o).

IR (Neat) 3084 (w), 2963 (w), 2934 (w), 2857 (w), 2839 (w), 2243 (w), 1640 (w), 1611 (m), 1511 (s), 1460 (m), 1416 (w), 1359 (w), 1244 (s), 1173 (m), 1087 (s), 1032 (s), 923 (m), 816 (s), 756 (w), $681(\mathrm{w}) \mathrm{cm}^{-1}$.

HRMS (EI, $\mathrm{M}^{+}$) calcd for $\mathrm{C}_{15} \mathrm{H}_{19} \mathrm{NO}_{2} 245.1416$, found 245.1408.

\section{3-(Hydroxymethyl)-3-methylpent-4-enenitrile (3o)}

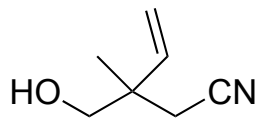

Color and State: Yellow oil; $b: l \geq 19: 1$

${ }^{1} \mathbf{H}$ NMR $\left(500 \mathrm{MHz}, \mathrm{CDCl}_{3}\right) \delta 5.81(\mathrm{dd}, J=17.6,11.0 \mathrm{~Hz}, 1 \mathrm{H}), 5.28(\mathrm{~d}, J=$ $10.9 \mathrm{~Hz}, 1 \mathrm{H}), 5.19(\mathrm{~d}, J=17.5 \mathrm{~Hz}, 1 \mathrm{H}), 3.53\left(\mathrm{~d}, \mathrm{~A}\right.$ of $\left.\mathrm{AB}, J_{\mathrm{AB}}=11.4 \mathrm{~Hz}, 1 \mathrm{H}\right), 3.51(\mathrm{~d}, \mathrm{~B}$ of $\mathrm{AB}$, $\left.J_{\mathrm{AB}}=11.3 \mathrm{~Hz}, 1 \mathrm{H}\right), 2.50(\mathrm{~s}, 2 \mathrm{H}), 1.54(\mathrm{~s}, 1 \mathrm{H}), 1.20(\mathrm{~s}, 3 \mathrm{H})$.

${ }^{13}$ C NMR (125 MHz, $\left.\mathrm{CDCl}_{3}\right) \delta 140.50$ (o), 118.15 (e), 115.99 (e), 68.45 (e), 41.18 (e), 25.28 (e), $21.03(0)$.

IR (Neat) 3428 (br, w), 3087 (w), 2968 (w), 2931 (w), 2875 (w), 2249 (w), 1642 (w), 1460 (w), $1416(\mathrm{~m}), 1380(\mathrm{w}), 1046(\mathrm{vs}), 923$ (s), $678(\mathrm{~m}) \mathrm{cm}^{-1}$.

HRMS (ESI, $[\mathrm{M}+\mathrm{H}]^{+}$) calcd for $\mathrm{C}_{7} \mathrm{H}_{12} \mathrm{NO} 126.0913$, found 126.0910. 


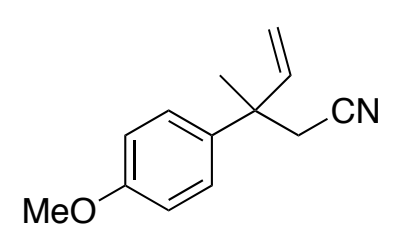

\section{3-(4-Methoxyphenyl)-3-methylpent-4-enenitrile (3p)}

Color and State: Yellow oil; $b: l \geq 19: 1$

${ }^{1} \mathbf{H}$ NMR $\left(500 \mathrm{MHz}, \mathrm{CDCl}_{3}\right) \delta$ 7.27-7.24 (m, 2H), 6.90-6.87 (m, 2H), 6.04 (dd, $J=17.4,10.7 \mathrm{~Hz}, 1 \mathrm{H}), 5.25$ (d, $J=10.8 \mathrm{~Hz}, 1 \mathrm{H}), 5.15$ (d, $J=17.4$

$\mathrm{Hz}, 1 \mathrm{H}), 3.80(\mathrm{~s}, 3 \mathrm{H}), 2.75\left(\mathrm{~d}, \mathrm{~A}\right.$ of $\left.\mathrm{AB}, J_{\mathrm{AB}}=16.4 \mathrm{~Hz}, 1 \mathrm{H}\right), 2.71\left(\mathrm{~d}, \mathrm{~B}\right.$ of $\left.\mathrm{AB}, J_{\mathrm{AB}}=16.6 \mathrm{~Hz}, 1 \mathrm{H}\right)$, $1.58(\mathrm{~s}, 3 \mathrm{H})$.

${ }^{13}$ C NMR (125 MHz, $\left.\mathrm{CDCl}_{3}\right) \delta 158.66$ (e), 143.67 (o), 135.82 (e), 127.40 (o), 118.07 (e), 114.05 (o), 113.85 (e), 55.40 (o), 42.71 (e), 30.41 (e), 25.78 (o).

IR (Neat) 3084 (w), 2966 (w), 2935 (w), 2837 (w), 2247 (w), 1637 (w), 1610 (w), 1513 (s), 1462 (w), 1296 (m), 1251 (s), 1184 (m), 1031 (m), 923 (w), 830 (m), 735 (w) cm $\mathrm{cm}^{-1}$.

HRMS (EI, $\mathrm{M}^{+}$) calcd for $\mathrm{C}_{13} \mathrm{H}_{15} \mathrm{NO} 201.1154$, found 201.1162 .

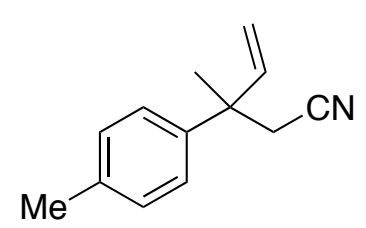

\section{3-Methyl-3-(p-tolyl)pent-4-enenitrile (3q)}

Color and State: Yellow oil; $b: l \geq 19: 1$

${ }^{1} \mathbf{H}$ NMR $\left(500 \mathrm{MHz}_{\mathrm{CDCl}}\right) \delta 7.22(\mathrm{~d}, J=8.3 \mathrm{~Hz}, 2 \mathrm{H}), 7.16(\mathrm{~d}, J=8.0$ Hz, 2H), 6.04 (dd, $J=17.4,10.7 \mathrm{~Hz}, 1 \mathrm{H}), 5.27$ (d, $J=10.7 \mathrm{~Hz}, 1 \mathrm{H}), 5.17$ $(\mathrm{d}, J=17.5 \mathrm{~Hz}, 1 \mathrm{H}), 2.76\left(\mathrm{~d}, \mathrm{~A}\right.$ of $\left.\mathrm{AB}, J_{\mathrm{AB}}=16.9 \mathrm{~Hz}, 1 \mathrm{H}\right), 2.73\left(\mathrm{~d}, \mathrm{~B}\right.$ of $\left.\mathrm{AB}, J_{\mathrm{AB}}=16.8 \mathrm{~Hz}, 1 \mathrm{H}\right)$, $2.34(\mathrm{~s}, 3 \mathrm{H}), 1.59(\mathrm{~s}, 3 \mathrm{H})$.

${ }^{13}$ C NMR (125 MHz, $\left.\mathrm{CDCl}_{3}\right) \delta 143.56$ (o), 140.89 (e), 136.96 (e), 129.46 (o), 126.13 (o), 118.07 (e), 114.00 (e), 42.98 (e), 30.29 (e), 25.67 (o), 21.04 (o).

IR (Neat) 3086 (w), 3024 (w), 2972 (w), 2923 (w), 2875 (w), 2244 (w), 1738 (w), 1637 (w), 1513 (m), 1455 (w), 1414 (m), 1376 (w), 998 (m), 920 (m), 813 (vs), 725 (m) cm cm $^{-1}$

HRMS (ESI, $[\mathrm{M}+\mathrm{H}]^{+}$) calcd for $\mathrm{C}_{13} \mathrm{H}_{16} \mathrm{~N}$ 186.1277, found 186.1272.

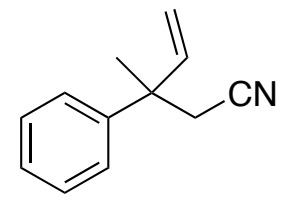

\section{3-Methyl-3-phenylpent-4-enenitrile (3r)}

Color and State: Colorless oil; $b: l \geq 19: 1$

${ }^{1} \mathbf{H}$ NMR $\left(500 \mathrm{MHz}, \mathrm{CDCl}_{3}\right) \delta$ 7.38-7.32 (m, 4H), 7.29-7.25 (m, 1H), $6.06(\mathrm{dd}$, $J=17.4,10.8 \mathrm{~Hz}, 1 \mathrm{H}), 5.29$ (d, $J=10.8 \mathrm{~Hz}, 1 \mathrm{H}), 5.19$ (d, $J=17.4 \mathrm{~Hz}, 1 \mathrm{H}), 2.79$ $\left(\mathrm{d}, \mathrm{A}\right.$ of $\left.\mathrm{AB}, J_{\mathrm{AB}}=16.5 \mathrm{~Hz}, 1 \mathrm{H}\right), 2.75\left(\mathrm{~d}, \mathrm{~B}\right.$ of $\left.\mathrm{AB}, J_{\mathrm{AB}}=16.5 \mathrm{~Hz}, 1 \mathrm{H}\right), 1.61(\mathrm{~s}, 3 \mathrm{H})$.

${ }^{13}$ C NMR (125 MHz, $\mathrm{CDCl}_{3}$ ) $\delta 143.85$ (e), 143.36 (o), 128.80 (o), 127.31 (o), 126.25 (o), 117.98 (e), 114.24 (e), 43.30 (e), 30.23 (e), 25.65 (o). 
IR (Neat) 3086 (w), 3059 (w), 3027 (w), 2973 (w), 2932 (w), 2881 (w), 2244 (w), 1637 (w), 1599 (w), $1494(\mathrm{w}), 1445(\mathrm{w}), 1413(\mathrm{w}), 1377(\mathrm{w}), 1028(\mathrm{w}), 998(\mathrm{w}), 921(\mathrm{~m}), 764$ (s), 697 (s) cm $\mathrm{cm}^{-1}$. HRMS (EI, $\mathrm{M}^{+}$) calcd for $\mathrm{C}_{12} \mathrm{H}_{13} \mathrm{~N}$ 171.1048, found 171.1040.

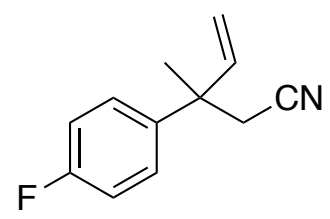

\section{3-(4-Fluorophenyl)-3-methylpent-4-enenitrile (3s)}

Color and State: Colorless oil; $b: l \geq 19: 1$

${ }^{1} \mathbf{H}$ NMR $\left(500 \mathrm{MHz}, \mathrm{CDCl}_{3}\right) \delta$ 7.33-7.28 (m, 2H), 7.06-7.02 (m, 2H), 6.03 $(\mathrm{dd}, J=17.6,10.8 \mathrm{~Hz}, 1 \mathrm{H}), 5.28(\mathrm{~d}, J=10.9 \mathrm{~Hz}, 1 \mathrm{H}), 5.17$ (d, $J=17.5 \mathrm{~Hz}$, $1 \mathrm{H}), 2.76\left(\mathrm{~d}, \mathrm{~A}\right.$ of $\left.\mathrm{AB}, J_{\mathrm{AB}}=16.5 \mathrm{~Hz}, 1 \mathrm{H}\right), 2.72\left(\mathrm{~d}, \mathrm{~B}\right.$ of $\left.\mathrm{AB}, J_{\mathrm{AB}}=16.5 \mathrm{~Hz}, 1 \mathrm{H}\right), 1.59(\mathrm{~s}, 3 \mathrm{H})$.

${ }^{13}$ C NMR $\left(125 \mathrm{MHz}, \mathrm{CDCl}_{3}\right) \delta 161.94\left(\mathrm{e}, \mathrm{d},{ }^{1} J_{\mathrm{CF}}=246.3 \mathrm{~Hz}\right), 143.24$ (o), $139.52\left(\mathrm{e}, \mathrm{d},{ }^{4} J_{\mathrm{CF}}=3.7\right.$ $\mathrm{Hz}), 128.06\left(\mathrm{o}, \mathrm{d},{ }^{3} J_{\mathrm{CF}}=8.2 \mathrm{~Hz}\right), 117.78(\mathrm{e}), 115.58\left(\mathrm{o}, \mathrm{d},{ }^{2} J_{\mathrm{CF}}=21.1 \mathrm{~Hz}\right), 114.35$ (e), $42.94(\mathrm{e})$, 30.41 (e), 25.87 (o).

${ }^{19}$ F NMR (471 MHz, $\left.\mathrm{CDCl}_{3}\right) \delta-115.60(\mathrm{~s})$.

IR (Neat) 3086 (w), 3050 (w), 2974 (w), 2934 (w), 2880 (w), 2246 (w), 1638 (w), 1600 (w), 1509 (s), 1461 (w), 1414 (w), 1378 (w), 1228 (s), 1165 (m), 1013 (w), 923 (m), 832 (vs), 729 (w) cm . $^{-1}$ HRMS (EI, $\mathrm{M}^{+}$) calcd for $\mathrm{C}_{12} \mathrm{H}_{12} \mathrm{FN} 189.0954$, found 189.0961 .

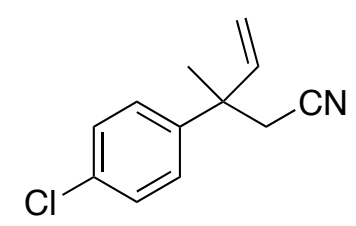

\section{3-(4-Chlorophenyl)-3-methylpent-4-enenitrile (3t)}

Color and State: Colorless oil; $b: l \geq 19: 1$

${ }^{1} \mathbf{H}$ NMR $\left(500 \mathrm{MHz}, \mathrm{CDCl}_{3}\right) \delta$ 7.34-7.31 (m, 2H), 7.28-7.26 (m, 2H), 6.02 $(\mathrm{dd}, J=17.5,10.7 \mathrm{~Hz}, 1 \mathrm{H}), 5.30(\mathrm{~d}, J=10.8 \mathrm{~Hz}, 1 \mathrm{H}), 5.17(\mathrm{~d}, J=17.4 \mathrm{~Hz}$,

$1 \mathrm{H}), 2.76\left(\mathrm{~d}, \mathrm{~A}\right.$ of $\left.\mathrm{AB}, J_{\mathrm{AB}}=16.6 \mathrm{~Hz}, 1 \mathrm{H}\right), 2.72\left(\mathrm{~d}, \mathrm{~B}\right.$ of $\left.\mathrm{AB}, J_{\mathrm{AB}}=16.7 \mathrm{~Hz}, 1 \mathrm{H}\right), 1.58(\mathrm{~s}, 3 \mathrm{H})$.

${ }^{13}$ C NMR (125 MHz, $\mathrm{CDCl}_{3}$ ) $\delta 142.92$ (o), 142.30 (e), 133.30 (e), 128.92 (o), 127.80 (o), 117.67 (e), 114.61 (e), 43.05 (e), 30.21 (e), 25.70 (o).

IR (Neat) 3087 (w), 2974 (w), 2936 (w), 2880 (w), 2245 (w), 1637 (w), 1595 (w), 1492 (s), 1414 (m), 1095 (s), 1010 (s), 922 (m), 823 (vs), 742 (m), 689 (w) cm $\mathrm{cm}^{-1}$.

HRMS (ESI, $[\mathrm{M}+\mathrm{H}]^{+}$) calcd for $\mathrm{C}_{12} \mathrm{H}_{13}{ }^{35} \mathrm{CIN} 206.0731$, found 206.0734. 


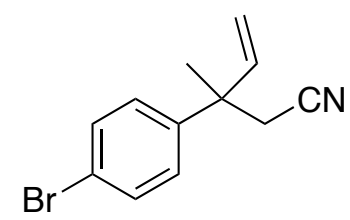

\section{3-(4-Bromophenyl)-3-methylpent-4-enenitrile (3u)}

Color and State: Yellow oil; $b: l \geq 19: 1$

${ }^{1} \mathbf{H}$ NMR $\left(500 \mathrm{MHz}, \mathrm{CDCl}_{3}\right) \delta$ 7.49-7.47 (m, 2H), 7.23-7.20 (m, 2H), 6.02 $(\mathrm{dd}, J=17.5,10.8 \mathrm{~Hz}, 1 \mathrm{H}), 5.30(\mathrm{~d}, J=10.7 \mathrm{~Hz}, 1 \mathrm{H}), 5.17(\mathrm{~d}, J=17.5 \mathrm{~Hz}$,

$1 \mathrm{H}), 2.75\left(\mathrm{~d}, \mathrm{~A}\right.$ of $\left.\mathrm{AB}, J_{\mathrm{AB}}=16.5 \mathrm{~Hz}, 1 \mathrm{H}\right), 2.72\left(\mathrm{~d}, \mathrm{~B}\right.$ of $\left.\mathrm{AB}, J_{\mathrm{AB}}=16.6 \mathrm{~Hz}, 1 \mathrm{H}\right), 1.58(\mathrm{~s}, 3 \mathrm{H})$.

${ }^{13}$ C NMR (125 MHz, $\left.\mathrm{CDCl}_{3}\right) \delta 142.84$ (o), 131.90 (o), 128.16 (o), 121.45 (e), 117.65 (e), 114.67 (e), 43.12 (e), 30.15 (e), 25.66 (o).

IR (Neat) 3086 (w), 2972 (w), 2933 (w), 2877 (w), 2245 (w), 1637 (w), 1588 (w), 1488 (m), 1414 (m), 1396 (m), 1081 (s), 1006 (s), 922 (m), 819 (s), 732 (m), 685 (w) cm $\mathrm{cm}^{-1}$.

HRMS (EI, $\mathrm{M}^{+}$) calcd for $\mathrm{C}_{12} \mathrm{H}_{12}{ }^{79} \mathrm{BrN} 249.0153$, found 249.0159.

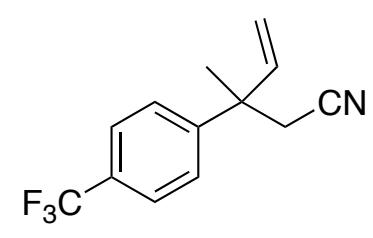

\section{3-Methyl-3-(4-(Trifluoromethyl)phenyl)pent-4-enenitrile (3v)}

Color and State: Yellow oil; $b: l \geq 19: 1$

${ }^{1} \mathbf{H}$ NMR $\left(500 \mathrm{MHz}, \mathrm{CDCl}_{3}\right) \delta$ 7.63-7.61 (m, 2H), 7.48-7.46 (m, 2H), $6.05(\mathrm{dd}, J=17.4,10.7 \mathrm{~Hz}, 1 \mathrm{H}), 5.34(\mathrm{~d}, J=10.8 \mathrm{~Hz}, 1 \mathrm{H}), 5.21(\mathrm{~d}, J=$

$17.4 \mathrm{~Hz}, 1 \mathrm{H}), 2.80\left(\mathrm{~d}, \mathrm{~A}\right.$ of $\left.\mathrm{AB}, J_{\mathrm{AB}}=16.7 \mathrm{~Hz}, 1 \mathrm{H}\right), 2.77\left(\mathrm{~d}, \mathrm{~B}\right.$ of $\left.\mathrm{AB}, J_{\mathrm{AB}}=16.7 \mathrm{~Hz}, 1 \mathrm{H}\right), 1.62$ $(\mathrm{s}, 3 \mathrm{H})$.

${ }^{13}$ C NMR (125 MHz, $\left.\mathrm{CDCl}_{3}\right) \delta 147.89$ (e), 142.65 (o), 129.79 (e, q, $\left.{ }^{2} J_{\mathrm{CF}}=32.7 \mathrm{~Hz}\right), 126.85$ (o), $125.81\left(\mathrm{o}, \mathrm{q},{ }^{3} J_{\mathrm{CF}}=3.7 \mathrm{~Hz}\right), 124.16\left(\mathrm{e}, \mathrm{q},{ }^{1} J_{\mathrm{CF}}=271.8 \mathrm{~Hz}\right), 117.39$ (e), $115.08(\mathrm{e}), 43.48(\mathrm{e}), 30.15$ (e), 25.72 (o).

${ }^{19}$ F NMR (471 MHz, $\left.\mathrm{CDCl}_{3}\right) \delta-62.64(\mathrm{~s})$.

IR (Neat) 3089 (w), 2977 (w), 2939 (w), 2883 (w), 2248 (w), 1639 (w), 1617 (w), 1459 (w), 1413 (w), 1324 (s), 1165 (m), 1114 (s), 1080 (s), 1013 (m), 926 (m), 836 (m), 708 (w) cm c $^{-1}$

HRMS (EI, $\mathrm{M}^{+}$) calcd for $\mathrm{C}_{13} \mathrm{H}_{12} \mathrm{~F}_{3} \mathrm{~N} 239.0922$, found 239.0915 .

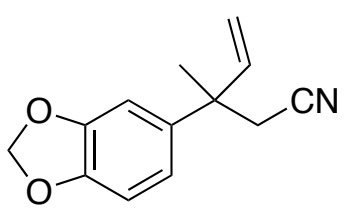

\section{3-(Benzo $[d][1,3]$ dioxol-5-yl)3-3-methylpent-4-enenitrile (3w)}

Color and State: Yellow oil; $b: l \geq 19: 1$

${ }^{1}$ H NMR $\left(500 \mathrm{MHz}, \mathrm{CDCl}_{3}\right) \delta$ 6.82-6.76 (m, 3H), 6.01 (dd, $J=17.5,10.8$ $\mathrm{Hz}, 1 \mathrm{H}), 5.95$ (s, 2H), 5.26 (d, $J=10.7 \mathrm{~Hz}, 1 \mathrm{H}), 5.16$ (d, $J=17.4 \mathrm{~Hz}$, $1 \mathrm{H}), 2.73\left(\mathrm{~d}, \mathrm{~A}\right.$ of $\left.\mathrm{AB}, J_{\mathrm{AB}}=16.6 \mathrm{~Hz}, 1 \mathrm{H}\right), 2.69\left(\mathrm{~d}, \mathrm{~B}\right.$ of $\left.\mathrm{AB}, J_{\mathrm{AB}}=16.5 \mathrm{~Hz}, 1 \mathrm{H}\right), 1.56(\mathrm{~s}, 3 \mathrm{H})$. 
${ }^{13}$ C NMR (125 MHz, $\left.\mathrm{CDCl}_{3}\right) \delta 148.07$ (e), 146.71 (e), 143.48 (o), 137.77 (e), 119.41 (o), 117.92 (e), 113.99 (e), 108.25 (o), 107.19 (o), 101.32 (e), 43.14 (e), 30.45 (e), 25.88 (o).

IR (Neat) 3083 (w), 2972 (w), 2893 (w), 2778 (w), 2245 (w), 1637 (w), 1609 (w), 1504 (m), 1485 (s), 1433 (m), 1346 (w), 1233 (vs), 1105 (m), 1034 (s), 926 (s), 807 (s), 730 (m), 681 (m) cm . $^{-1}$

HRMS (EI, $\mathrm{M}^{+}$) calcd for $\mathrm{C}_{13} \mathrm{H}_{13} \mathrm{NO}_{2} 215.0946$, found 215.0939.

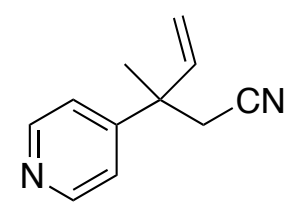

\section{3-Methyl-3-(pyridin-4-yl)pent-4-enenitrile (3x)}

Color and State: Yellow oil; $b: l \geq 19: 1$

${ }^{1}$ H NMR $\left(500 \mathrm{MHz}, \mathrm{CDCl}_{3}\right) \delta 8.61(\mathrm{dd}, J=4.4,1.7 \mathrm{~Hz}, 2 \mathrm{H}), 7.25(\mathrm{dd}, J=4.6$, $1.7 \mathrm{~Hz}, 2 \mathrm{H}), 6.02$ (dd, $J=17.4,10.7 \mathrm{~Hz}, 1 \mathrm{H}), 5.36$ (d, $J=10.7 \mathrm{~Hz}, 1 \mathrm{H}), 5.22$ $(\mathrm{d}, J=17.4 \mathrm{~Hz}, 1 \mathrm{H}), 2.79\left(\mathrm{~d}, \mathrm{~A}\right.$ of $\left.\mathrm{AB}, J_{\mathrm{AB}}=16.6 \mathrm{~Hz}, 1 \mathrm{H}\right), 2.75\left(\mathrm{~d}, \mathrm{~B}\right.$ of $\left.\mathrm{AB}, J_{\mathrm{AB}}=16.6 \mathrm{~Hz}, 1 \mathrm{H}\right)$, $1.60(\mathrm{~s}, 3 \mathrm{H})$.

${ }^{13}$ C NMR (125 MHz, $\mathrm{CDCl}_{3}$ ) $\delta 152.64$ (e), 150.44 (o), 141.70 (o), 121.42 (o), 117.22 (e), 115.69 (e), 43.12 (e), 29.57 (e), 25.11 (o).

IR (Neat) 3082 (w), 3025 (w), 2976 (w), 2929 (w), 2244 (w), 1637 (w), 1594 (s), 1552 (w), 1458 (w), 1411 (s), 1070 (m), 995 (m), 926 (s), 817 (vs), 749 (w), 685 (s) cm $\mathrm{cm}^{-1}$.

HRMS (EI, $\mathrm{M}^{+}$) calcd for $\mathrm{C}_{11} \mathrm{H}_{12} \mathrm{~N}_{2}$ 172.1000, found 172.1009.

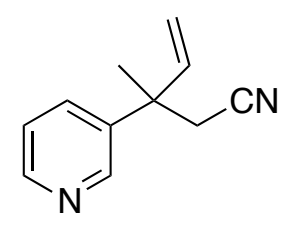

\section{3-Methyl-3-(pyridin-3-yl)pent-4-enenitrile (3y)}

Color and State: Yellow oil; $b: l \geq 19: 1$

${ }^{1} \mathbf{H}$ NMR $\left(500 \mathrm{MHz}, \mathrm{CDCl}_{3}\right) \delta 8.63(\mathrm{dd}, J=2.5,0.6 \mathrm{~Hz}, 1 \mathrm{H}), 8.54(\mathrm{dd}, J=$ 4.8, $1.4 \mathrm{~Hz}, 1 \mathrm{H}), 7.66$ (ddd, $J=8.0,2.6,1.5 \mathrm{~Hz}, 1 \mathrm{H}), 7.29$ (ddd, $J=8.0,4.8$, $0.9 \mathrm{~Hz}, 1 \mathrm{H}), 6.05(\mathrm{dd}, J=17.4,10.7 \mathrm{~Hz}, 1 \mathrm{H}), 5.35$ (d, $J=10.9 \mathrm{~Hz}, 1 \mathrm{H}), 5.21$ (d, $J=17.5 \mathrm{~Hz}$, $1 \mathrm{H}), 2.81\left(\mathrm{~d}, \mathrm{~A}\right.$ of $\left.\mathrm{AB}, J_{\mathrm{AB}}=16.7 \mathrm{~Hz}, 1 \mathrm{H}\right), 2.77\left(\mathrm{~d}, \mathrm{~B}\right.$ of $\left.\mathrm{AB}, J_{\mathrm{AB}}=16.7 \mathrm{~Hz}, 1 \mathrm{H}\right), 1.63(\mathrm{~s}, 3 \mathrm{H})$.

${ }^{13}$ C NMR (125 MHz, $\mathrm{CDCl}_{3}$ ) $\delta 148.73$ (o), 148.18 (o), 142.22 (o), 139.13 (e), 134.05 (o), 123.49 (o), 117.36 (e), 115.31 (e), 42.15 (e), 30.07 (e), 25.50 (o).

IR (Neat) 3087 (w), 3036 (w), 2974 (w), 2243 (w), 1638 (w), 1573 (w), 1480 (m), 1415 (s), 1380 (w), $1086(\mathrm{w}), 1022(\mathrm{~m}), 927(\mathrm{~m}), 810(\mathrm{~m}), 714(\mathrm{vs}) \mathrm{cm}^{-1}$.

HRMS $\left(E I, M^{+}\right)$calcd for $\mathrm{C}_{11} \mathrm{H}_{12} \mathrm{~N}_{2}$ 172.1000, found 172.1009. 


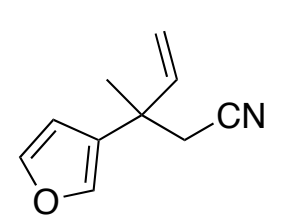

\section{3-(Furan-3-yl)-3-methylpent-4-enenitrile (3z)}

Color and State: Yellow oil; $b: l \geq 19: 1$

${ }^{1} \mathbf{H}$ NMR $\left(500 \mathrm{MHz}^{\left.\mathrm{CDCl}_{3}\right)} \delta 7.41\right.$ (app. t, $\left.J=1.6 \mathrm{~Hz}, 1 \mathrm{H}\right), 7.29$ (dd, $J=1.4$, $1.0 \mathrm{~Hz}, 1 \mathrm{H}), 6.33(\mathrm{dd}, J=1.8,0.9 \mathrm{~Hz}, 1 \mathrm{H}), 6.01(\mathrm{dd}, J=17.4,10.7 \mathrm{~Hz}, 1 \mathrm{H}), 5.20$ (d, $J=10.7 \mathrm{~Hz}, 1 \mathrm{H}), 5.13(\mathrm{~d}, J=17.3 \mathrm{~Hz}, 1 \mathrm{H}), 2.64(\mathrm{~s}, 2 \mathrm{H}), 1.52(\mathrm{~s}, 3 \mathrm{H})$.

${ }^{13}$ C NMR (125 MHz, $\mathrm{CDCl}_{3}$ ) $\delta 143.73$ (o), 142.46 (o), 138.80 (o), 129.63 (e), 117.73 (e), 114.43 (e), 109.03 (o), 38.23 (e), 30.43 (e), 25.44 (o)

IR (Neat) 3144 (w), 3088 (w), 2974 (w), 2936 (w), 2876 (w), 2247 (w), 1738 (w), 1639 (w), 1503 (w), 1458 (w), 1414 (w), 1376 (w), 1163 (m), 1025 (m), 925 (m), 873 (s), 789 (vs), 730 (m), 664 (m) $\mathrm{cm}^{-1}$.

HRMS (ESI, $[\mathrm{M}+\mathrm{H}]^{+}$) calcd for $\mathrm{C}_{10} \mathrm{H}_{12} \mathrm{NO} 162.0913$, found 162.0914.

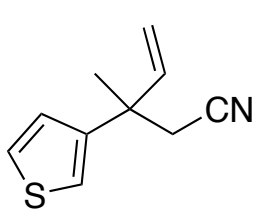

\section{3-Methyl-3-(thiophen-3-yl)pent-4-enenitrile (3aa)}

Color and State: Yellow oil; $b: l \geq 19: 1$

${ }^{1} \mathbf{H}$ NMR $\left(500 \mathrm{MHz}, \mathrm{CDCl}_{3}\right) \delta 7.33(\mathrm{dd}, J=5.0,2.9 \mathrm{~Hz}, 1 \mathrm{H}), 7.10(\mathrm{dd}, J=3.0$, $1.5 \mathrm{~Hz}, 1 \mathrm{H}), 7.02(\mathrm{dd}, J=5.1,1.4 \mathrm{~Hz}, 1 \mathrm{H}), 6.06(\mathrm{dd}, J=17.4,10.7 \mathrm{~Hz}, 1 \mathrm{H}), 5.23$

(d, $J=10.7 \mathrm{~Hz}, 1 \mathrm{H}), 5.13(\mathrm{~d}, J=17.3 \mathrm{~Hz}, 1 \mathrm{H}), 2.73(\mathrm{~s}, 2 \mathrm{H}), 1.60$ (s, 3H).

${ }^{13}$ C NMR (125 MHz, $\mathrm{CDCl}_{3}$ ) $\delta 145.43$ (e), 142.91 (o), 126.47 (o), 126.20 (o), 120.78 (o), 117.80 (e), 114.16 (e), 41.44 (e), 30.42 (e), 25.75 (o).

IR (Neat) 3104 (w), 3088 (w), 2973 (w), 2933 (w), 2876 (w), 2245 (w), 1737 (w), 1637 (w), 1457 (w), $1412(\mathrm{~m}), 1373(\mathrm{w}), 1088(\mathrm{w}), 996$ (m), 922 (m), 866 (m), 781 (vs), $694(\mathrm{~m}) \mathrm{cm}^{-1}$.

HRMS (EI, $\mathrm{M}^{+}$) calcd for $\mathrm{C}_{10} \mathrm{H}_{11} \mathrm{NS} 177.0612$, found 177.0619 .

\section{Stereospecific Rhodium-Catalyzed Allylic Cyanomethylation of $(R)-2 q$ to $(R)-3 q$}

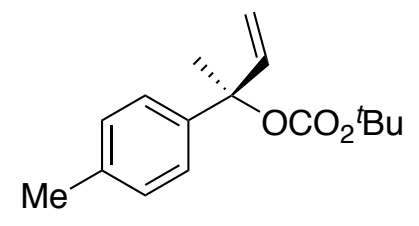

(R)-tert-Butyl (2-(p-tolyl)but-3-en-2-yl) carbonate $(R)-(2 q)$

Prepared according to the general procedure (Method A) from the previously reported preparation of the enriched alcohol. ${ }^{4,7}$ Color and State: Pale yellow oil; $[\alpha]_{\mathrm{D}}^{20}+9.4\left(c=1.0, \mathrm{CHCl}_{3}\right)$.

Chiral HPLC analysis of the tertiary alcohol (CHIRALCEL OJ-H column), 98:2 hexane/isopropanol at $1.0 \mathrm{~mL} / \mathrm{min}$. flow rate; $t_{\mathrm{R}}(S)$-enantiomer (minor) $=21.7 \mathrm{~min}$., $t_{\mathrm{R}}(R)$ - 
enantiomer (major) $=31.3$ min.; $99 \% e e .[\alpha]_{\mathrm{D}}^{20}+28.2\left(c=1.0, \mathrm{CHCl}_{3}\right)$ for the $(R)$-tertiary alcohol; Lit. $[\alpha]_{\mathrm{D}}^{20}+26.2\left(c=1.0, \mathrm{CHCl}_{3}\right)^{4}$

${ }^{1}$ H NMR $\left(500 \mathrm{MHz}, \mathrm{CDCl}_{3}\right) \delta$ 7.28-7.25 (m, 2H), 7.15-7.13 (m, 2H), $6.33(\mathrm{dd}, J=17.4,10.8 \mathrm{~Hz}$, 1H), 5.27 (dd, $J=17.5,0.8 \mathrm{~Hz}, 1 \mathrm{H}), 5.25$ (dd, $J=10.8,0.8 \mathrm{~Hz}, 1 \mathrm{H}), 2.33$ (s, 3H), $1.86(\mathrm{~s}, 3 \mathrm{H})$, $1.42(\mathrm{~s}, 9 \mathrm{H})$.

IR (Neat) 3091 (w), 2979 (w), 2932 (w), 2872 (w), 1742 (s), 1641 (w), 1367 (m), 1274 (s), 1252 (s), 1153 (s), 1093 (s), $924(\mathrm{~m}), 850(\mathrm{~m}), 815(\mathrm{~s}), 791(\mathrm{~m}), 694(\mathrm{w}) \mathrm{cm}^{-1}$.

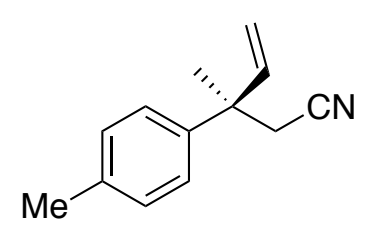

(R)-4-Methyl-3-(p-tolyl)pent-4-enenitrile (( $R)$-3q)

Color and State: Yellow oil; $b: l \geq 19: 1 ;[\alpha]_{\mathrm{D}}^{20}+12.6\left(c=1.0, \mathrm{CHCl}_{3}\right)$.

Chiral HPLC analysis (CHIRALCEL OJ-H column), 99:1 hexane/isopropanol at $0.8 \mathrm{~mL} / \mathrm{min}$. flow rate; $t_{\mathrm{R}}(S)$-enantiomer $($ minor $)=$ 24.9 min., $t_{\mathrm{R}}(R)$-enantiomer (major) $=30.9$ min.; $99 \%$ ee .

${ }^{1} \mathbf{H}$ NMR $\left(500 \mathrm{MHz}, \mathrm{CDCl}_{3}\right) \delta 7.22(\mathrm{~d}, J=8.2 \mathrm{~Hz}, 2 \mathrm{H}), 7.16(\mathrm{~d}, J=8.1 \mathrm{~Hz}, 2 \mathrm{H}), 6.04(\mathrm{dd}, J=$ 17.4, $10.7 \mathrm{~Hz}, 1 \mathrm{H}), 5.26(\mathrm{~d}, J=10.7 \mathrm{~Hz}, 1 \mathrm{H}), 5.17(\mathrm{~d}, J=17.5 \mathrm{~Hz}, 1 \mathrm{H}), 2.76\left(\mathrm{~d}, \mathrm{~A}\right.$ of AB, $J_{\mathrm{AB}}=$ $16.9 \mathrm{~Hz}, 1 \mathrm{H}), 2.73\left(\mathrm{~d}, \mathrm{~B}\right.$ of AB, $\left.J_{\mathrm{AB}}=16.8 \mathrm{~Hz}, 1 \mathrm{H}\right), 2.34(\mathrm{~s}, 3 \mathrm{H}), 1.58(\mathrm{~s}, 3 \mathrm{H})$.

IR (Neat) 3086 (w), 3023 (w), 2973 (w), 2923 (w), 2875 (w), 2244 (w), 1637 (w), 1513 (m), 1455 (w), $1413(\mathrm{~m}), 1376(\mathrm{w}), 998(\mathrm{~m}), 920(\mathrm{~m}), 813(\mathrm{vs}), 725(\mathrm{~m}) \mathrm{cm}^{-1}$.

\section{Derivatizations of the Enantioenriched Nitrile $(R)-3 q$ to $5-7$}

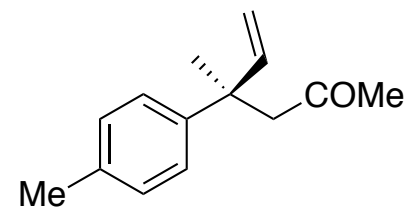

(R)-4-Methyl-4-(p-tolyl)hex-5-en-2-one (5). Methylmagnesium bromide ( $0.21 \mathrm{~mL}, 0.63 \mathrm{mmol} ; 3 \mathrm{M}$ in diethyl ether) was added dropwise to a stirring solution of $(R)$-3-methyl-3-( $p$-tolyl)pent-4-enenitrile $(R)-\mathbf{3 q}$ (46 $\mathrm{mg}, 0.25 \mathrm{mmol})$ in toluene $(2.5 \mathrm{~mL})$ at room temperature. The resulting solution was heated to $110{ }^{\circ} \mathrm{C}$ for $c a .16$ hours. The mixture was then allowed to cool to room temperature and quenched with water. A solution of acetic acid, tetrahydrofuran and water (4:1:1) was then added and the resulting biphasic mixture stirred vigorously for $c a .4$ hours. The mixture was then partitioned between ethyl acetate and $2 \mathrm{M}$ aq. $\mathrm{NaOH}$. The organic layers were combined, washed with sat. aq $\mathrm{NaHCO}_{3}$, brine, dried (anhyd. $\mathrm{MgSO}_{4}$ ), filtered and concentrated in vacuo to afford the crude product. Purification by flask column chromatography (silica gel, eluting with $2-8 \%$ diethyl ether/hexanes) afforded the methyl ketone $5(35.8 \mathrm{mg}, 71 \%)$ as a yellow 
oil: $[\alpha]_{\mathrm{D}}^{20}+18.0\left(c=1.46, \mathrm{CHCl}_{3}\right)$; Lit. $[\alpha]_{\mathrm{D}}^{22}-20.7\left(c=1.46, \mathrm{CHCl}_{3}\right)^{8}$ for the $(S)$-enantiomer $){ }^{\dagger}$ Chiral HPLC analysis (CHIRALCEL OJ-H column), 99:1 hexane/isopropanol at $0.8 \mathrm{~mL} / \mathrm{min}$. flow rate; $t_{\mathrm{R}}(S)$-enantiomer $($ minor $)=47.4 \mathrm{~min}$., $t_{\mathrm{R}}(R)$-enantiomer $($ major $)=70.6 \mathrm{~min}$.; $99 \%$ ee .

${ }^{1} \mathrm{H}$ NMR $\left(500 \mathrm{MHz}, \mathrm{CDCl}_{3}\right) \delta$ 7.21-7.19 (m, 2H), 7.13-7.11 (m, 2H), $6.12(\mathrm{dd}, J=17.5,10.7 \mathrm{~Hz}$, $1 \mathrm{H}), 5.12(\mathrm{~d}, J=10.6 \mathrm{~Hz}, 1 \mathrm{H}), 5.04(\mathrm{~d}, J=17.4 \mathrm{~Hz}, 1 \mathrm{H}), 2.93\left(\mathrm{~d}, \mathrm{~A}\right.$ of $\left.\mathrm{AB}, J_{\mathrm{AB}}=14.4 \mathrm{~Hz}, 1 \mathrm{H}\right)$, $2.84\left(\mathrm{~d}, \mathrm{~B}\right.$ of $\left.\mathrm{AB}, J_{\mathrm{AB}}=14.3 \mathrm{~Hz}, 1 \mathrm{H}\right), 2.32(\mathrm{~s}, 3 \mathrm{H}), 1.89(\mathrm{~s}, 3 \mathrm{H}), 1.49$ (s, 3H).

IR (Neat) 3083 (w), 3021 (w), 2965 (w), 2923 (w), 2874 (w), 1703 (s), 1634 (w), 1512 (m), 1454 (m), 1412 (m), 1354 (s), 1157 (m), 1018 (m), 913 (s), 814 (vs), 726 (m) cm .

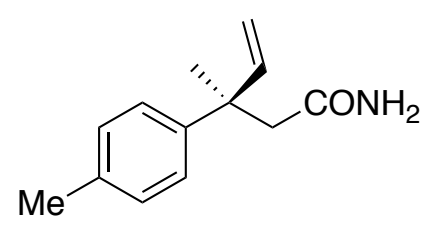

(R)-3-Methyl-3-(p-tolyl)pent-4-enamide (6). Hydrogen peroxide $(56.0 \mu \mathrm{L}, 0.55 \mathrm{mmol} ; 30 \%(\mathrm{w} / \mathrm{w})$ solution in water) and potassium carbonate $(5.5 \mathrm{mg}, 0.04 \mathrm{mmol})$ were added to a stirred solution of $(R)$ 3-methyl-3-( $p$-tolyl)pent-4-enenitrile $(R)$-3q (46.0 mg, $0.25 \mathrm{mmol})$ in dimethyl sulfoxide $(0.5 \mathrm{~mL})$ at $0{ }^{\circ} \mathrm{C}$. The resulting solution was allowed to slowly warm to room temperature and stirred until completion (TLC control). The reaction was quenched by the addition of water and extracted with ethyl acetate. The organic phases were combined, dried (anhyd. $\mathrm{MgSO}_{4}$ ), filtered and concentrated in vacuo to afford the crude product. Purification by flask column chromatography (silica gel, eluting with $80-100 \%$ ethyl acetate/hexane) afforded the primary amide $6(44.1 \mathrm{mg}, 87 \%)$ as a colorless oil: $[\alpha]_{\mathrm{D}}^{20}+40.2\left(c=1.0, \mathrm{CHCl}_{3}\right)$; Chiral HPLC analysis (CHIRALCEL OJ-H column), 95:5 hexane/isopropanol at $0.8 \mathrm{~mL} / \mathrm{min}$ flow rate; $t_{\mathrm{R}}(S)$ enantiomer $(\operatorname{minor})=32.8 \mathrm{~min}$., $t_{\mathrm{R}}(R)$-enantiomer $($ major $)=57.3 \mathrm{~min} ., 99 \%$ ee.

${ }^{1} \mathbf{H}$ NMR $\left(500 \mathrm{MHz}, \mathrm{CDCl}_{3}\right) \delta$ 7.28-7.25 (m, 2H), 7.17-7.14 (m, 2H), $6.12(\mathrm{dd}, J=17.5,10.8 \mathrm{~Hz}$, 1H), 5.16 (dd, $J=10.8,0.7 \mathrm{~Hz}, 1 \mathrm{H}), 5.10$ (dd, $J=17.5,0.6 \mathrm{~Hz}, 1 \mathrm{H}), 5.08$ (br s, 1H), 4.89 (br s, $1 \mathrm{H}), 2.75\left(\mathrm{~d}, \mathrm{~A}\right.$ of $\left.\mathrm{AB}, J_{\mathrm{AB}}=13.7 \mathrm{~Hz}, 1 \mathrm{H}\right), 2.68\left(\mathrm{~d}, \mathrm{~B}\right.$ of $\left.\mathrm{AB}, J_{\mathrm{AB}}=13.7 \mathrm{~Hz}, 1 \mathrm{H}\right), 2.32(\mathrm{~s}, 3 \mathrm{H})$, $1.54(\mathrm{~s}, 3 \mathrm{H})$.

${ }^{13}$ C NMR (125 MHz, $\left.\mathrm{CDCl}_{3}\right) \delta 173.21$ (e), 146.07 (o), 142.69 (e), 136.43 (e), 129.49 (o), 126.59 (o), 112.75 (e), 48.32 (e), 43.25 (e), 25.23 (o), 21.03 (o).

IR (Neat) 3458 (w), 3323 (br w), 3187 (w), 3084 (w), 3018 (w), 2969 (w), 2922 (w), 2872 (w), 1656 (vs), 1608 (m), 1511 (m), 1447 (m), 1391 (m), 1258 (m), 1018 (m), 912 (m), 814 (s) cm-1.

\footnotetext{
${ }^{\dagger}$ There is a typographical error in the SI, which wrongly labeled the methyl ketone $\mathbf{5}$ as the $(R)$-enantiomer.
} 
HRMS (ESI, $[\mathrm{M}+\mathrm{H}]^{+}$) calcd for $\mathrm{C}_{13} \mathrm{H}_{18} \mathrm{NO} 204.1383$, found 204.1381.

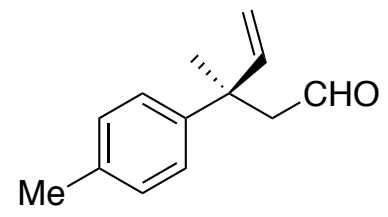

(R)-3-Methyl-3-(p-tolyl)pent-4-enal (7). Diisobutylaluminum hydride $(0.50 \mathrm{~mL}, 0.50 \mathrm{mmol} ; 1 \mathrm{M}$ solution in toluene) was added dropwise to a stirring solution of $(R)$-3-methyl-3-( $p$-tolyl)pent-4-enenitrile $(R)$-3q (46.0 $\mathrm{mg}, 0.25 \mathrm{mmol})$ in dichloromethane $(0.50 \mathrm{~mL})$ at $0{ }^{\circ} \mathrm{C}$. The resulting solution was allowed to stir for $c a .2$ hours before being quenched by the addition of methanol and allowed to warm to room temperature. The resulting mixture was partitioned between ethyl acetate and water. The combined organic phases were washed with brine, dried (anhyd. $\mathrm{MgSO}_{4}$ ), filtered and concentrated in vacuo to afford the crude product. Purification by flask column chromatography (silica gel, eluting with 5-15\% diethyl ether/pentane) afforded the aldehyde 7 (32.4 mg, 69\%) as a colorless oil: $[\alpha]_{\mathrm{D}}^{20}-3.7\left(c=1.0, \mathrm{CHCl}_{3}\right)$; Lit. $[\alpha]_{\mathrm{D}}^{20}-1.2\left(c=1.1, \mathrm{CH}_{2} \mathrm{Cl}_{2}\right)^{9}$ for the $(R)$-enantiomer and $[\alpha]_{\mathrm{D}}+5.2\left(c=1.0, \mathrm{CHCl}_{3}\right)^{10}$ for the $(S)$-enantiomer; Chiral HPLC analysis (CHIRALCEL OJ-H column), 99:1 hexane/isopropanol at $0.8 \mathrm{~mL} / \mathrm{min}$ flow rate; $t_{\mathrm{R}}(S)$ enantiomer $($ minor $)=19.5 \mathrm{~min} ., t_{\mathrm{R}}(R)$-enantiomer $($ major $)=25.0 \mathrm{~min}$., $99 \%$ ee .

${ }^{1} \mathbf{H}$ NMR $\left(500 \mathrm{MHz}, \mathrm{CDCl}_{3}\right) \delta 9.58(\mathrm{t}, J=2.9 \mathrm{~Hz}, 1 \mathrm{H}), 7.23-7.21(\mathrm{~m}, 2 \mathrm{H}), 7.16-7.14(\mathrm{~m}, 2 \mathrm{H})$, $6.10(\mathrm{dd}, J=17.4,10.7 \mathrm{~Hz}, 1 \mathrm{H}), 5.20(\mathrm{dd}, J=10.7,0.5 \mathrm{~Hz}, 1 \mathrm{H}), 5.11$ (d, $J=17.6 \mathrm{~Hz}, 1 \mathrm{H}), 2.81$ $\left(\mathrm{dd}, \mathrm{A}\right.$ of $\left.\mathrm{ABX}, J_{\mathrm{AB}}=15.3 \mathrm{~Hz}, J_{\mathrm{AX}}=2.9 \mathrm{~Hz}, 1 \mathrm{H}\right), 2.74\left(\mathrm{dd}, J_{\mathrm{AB}}=15.3 \mathrm{~Hz}, J_{\mathrm{BX}}=2.9 \mathrm{~Hz}, 1 \mathrm{H}\right), 2.33$ (s, 3H), $1.52(\mathrm{~s}, 3 \mathrm{H})$.

${ }^{13}$ C NMR (125 MHz, $\mathrm{CDCl}_{3}$ ) $\delta 203.12$ (o), 145.38 (o), 142.60 (e), 136.37 (e), 129.41 (o), 126.31 (o), 113.07 (e), 53.43 (e), 42.70 (e), 26.29 (o), 21.01 (o).

IR (Neat) 3085 (w), 3022 (w), 2967 (w), 2922 (w), 2873 (w) 2834 (w), 2736 (w), 1718 (vs), 1635 (w), $1512(\mathrm{~m}), 1454(\mathrm{w}), 1410(\mathrm{~m}), 1374(\mathrm{w}), 1018(\mathrm{~m}), 916(\mathrm{~m}), 814(\mathrm{~s}), 723(\mathrm{~m}) \mathrm{cm}^{-1}$.

HRMS (EI, M ${ }^{+}$) calcd for $\mathrm{C}_{13} \mathrm{H}_{16} \mathrm{O} 188.1201$, found 188.1209.

\section{References}

1. Suzuki, E.; Komuro, T.; Okazaki, M.; Tobita, H. Three- and Five-Membered W/C/N Metallacycles Formed by Incorporation of Acetonitrile Molecules into Silyltungsten Intermediates. Organometallics 2007, 26, 4379. 
2. Furuta, K.; Ishiguro, M.; Haruta, R.; Ikeda, N.; Yamamoto, H. Regio- and Stereocontrolled Synthesis of Allenic and Acetylenic Derivatives. Organotitanium and Boron Reagents. Bull. Chem. Soc. Jpn. 1984, 57, 2768.

3. Evans, P. A; Oliver, S.; Chae, J. Rhodium-Catalyzed Allylic Substitution with an Acyl Anion Equivalent: Stereospecific Construction of Acyclic Quaternary Carbon Stereogenic Centers. $J$. Am. Chem. Soc. 2012, 134, 19314.

4. Evans, P. A.; Oliver, S. Regio- and Enantiospecific Rhodium-Catalyzed Allylic Substitution with an Acyl Anion Equivalent. Org. Lett. 2013, 15, 5626.

5. Zhang, P.; Le, H.; Kyne, R. E.; Morken, J. P. Enantioselective Construction of All-Carbon Quaternary Centers by Branch-Selective Pd-Catalyzed Allyl-Allyl Cross-Coupling. J. Am. Chem. Soc. 2011, 133, 9716.

6. Guo, W.; Cai, A.; Xie, J.; Kleig, A. W. Asymmetric Synthesis of $\alpha, \alpha$-Disubstituted Allylic Amines Through Palladium-Catalyzed Allylic Substitution. Angew. Chem., Int. Ed. 2017, 56, 11797.

7. (a) Stymiest, J. L.; Bagutski, V.; French, R. M.; Aggarwal, V. K. Enantiodivergent Conversion of Chiral Secondary Alcohols into Tertiary Alcohols. Nature 2008, 456, 778. (b) Bagutski, V.; French, R. M.; Aggarwal, V. K. Full Chirality Transfer in the Conversion of Secondary Alcohols into Tertiary Boronic Esters and Alcohols using Lithiation-Borylation Reactions. Angew. Chem., Int. Ed. 2010, 49, 5142.

8. Jung, B.; Hoveyda, A. H. Site- and Enantioselective Formation of Allene-Bearing Tertiary or Quaternary Carbon Stereogenic Centers Through NHC-Cu-Catalyzed Allylic Substitution. J. Am. Chem. Soc. 2012, 134, 1490.

9. Kametani, T.; Kawamura, K.; Tsubuki, M.; Honda, T. Synthetic Approach to (-)-Cuparenone by Rhodium-Catalyzed Cyclization. Chem. Pharm. Bull. 1985, 33, 4821.

10. Fadel, A.; Canet, J.-L.; Salaün, J. Asymmetric Construction of Quaternary Carbons from Chiral Malonates: Total Syntheses of (+)-Epilaurene and (-)-Isolaurene. Tetrahedron: Asymmetry $1993,4,27$. 
14. Copies of Proton and Carbon NMR Spectra

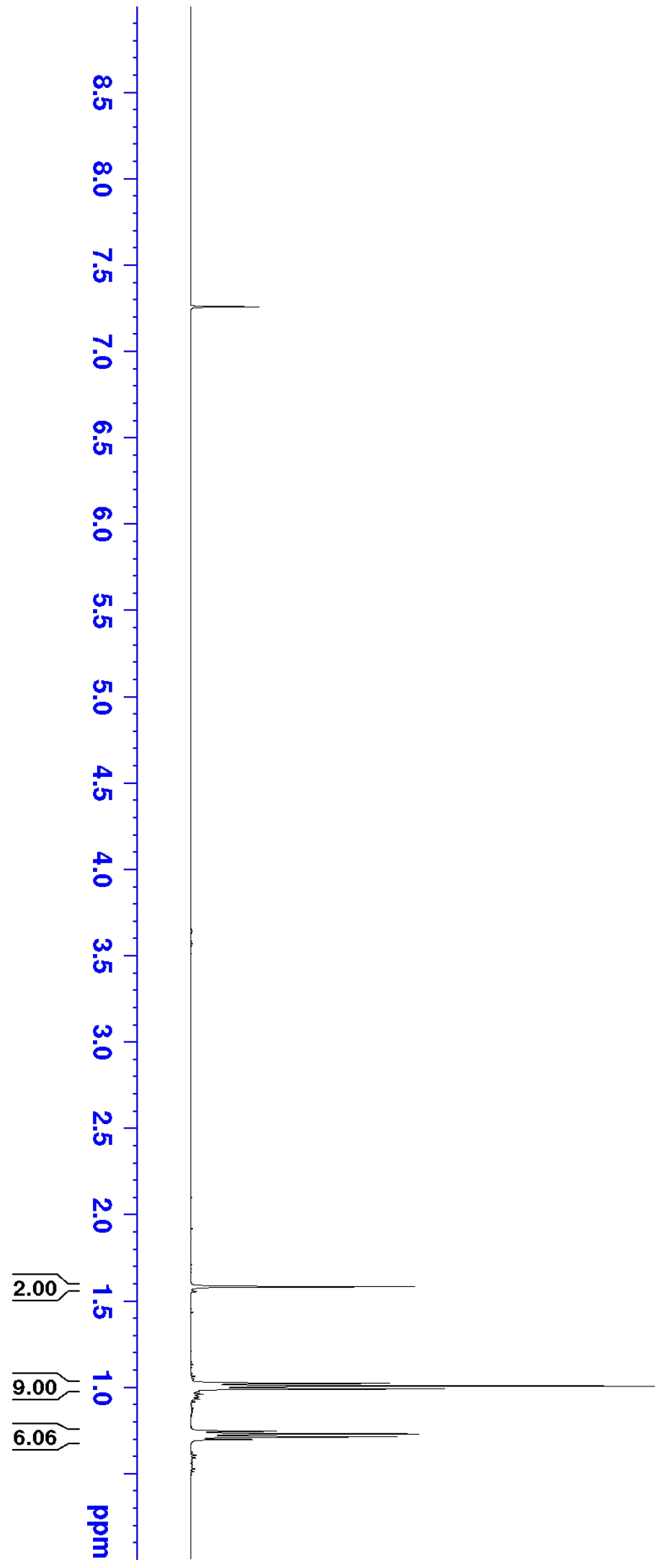

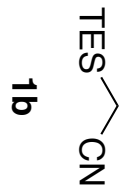




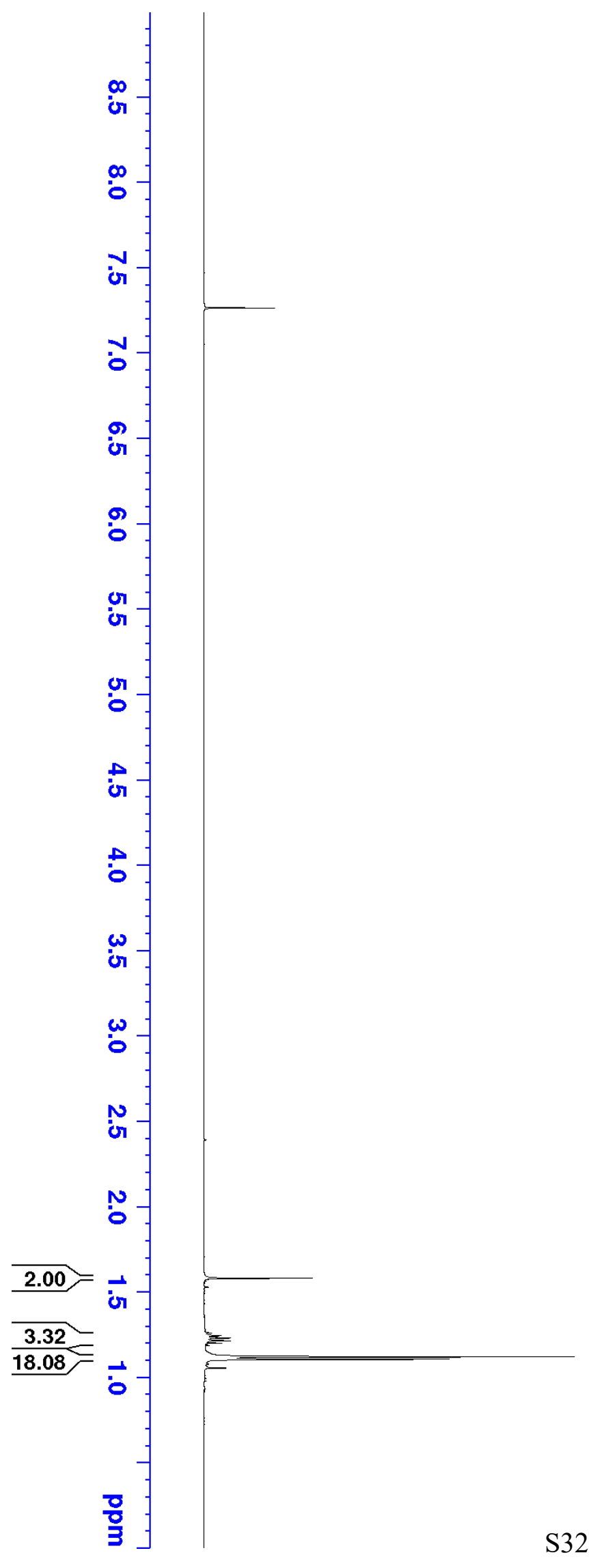




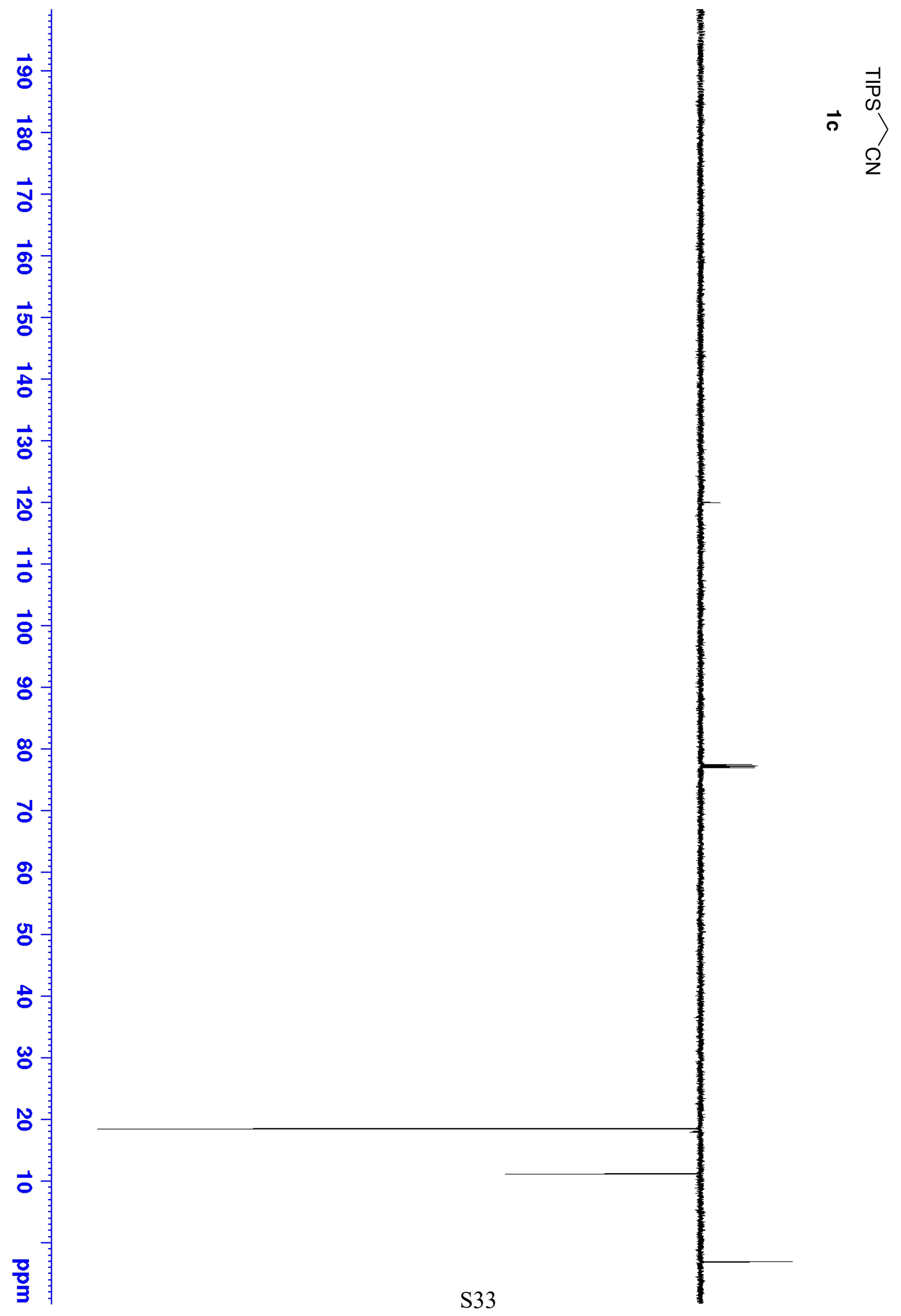




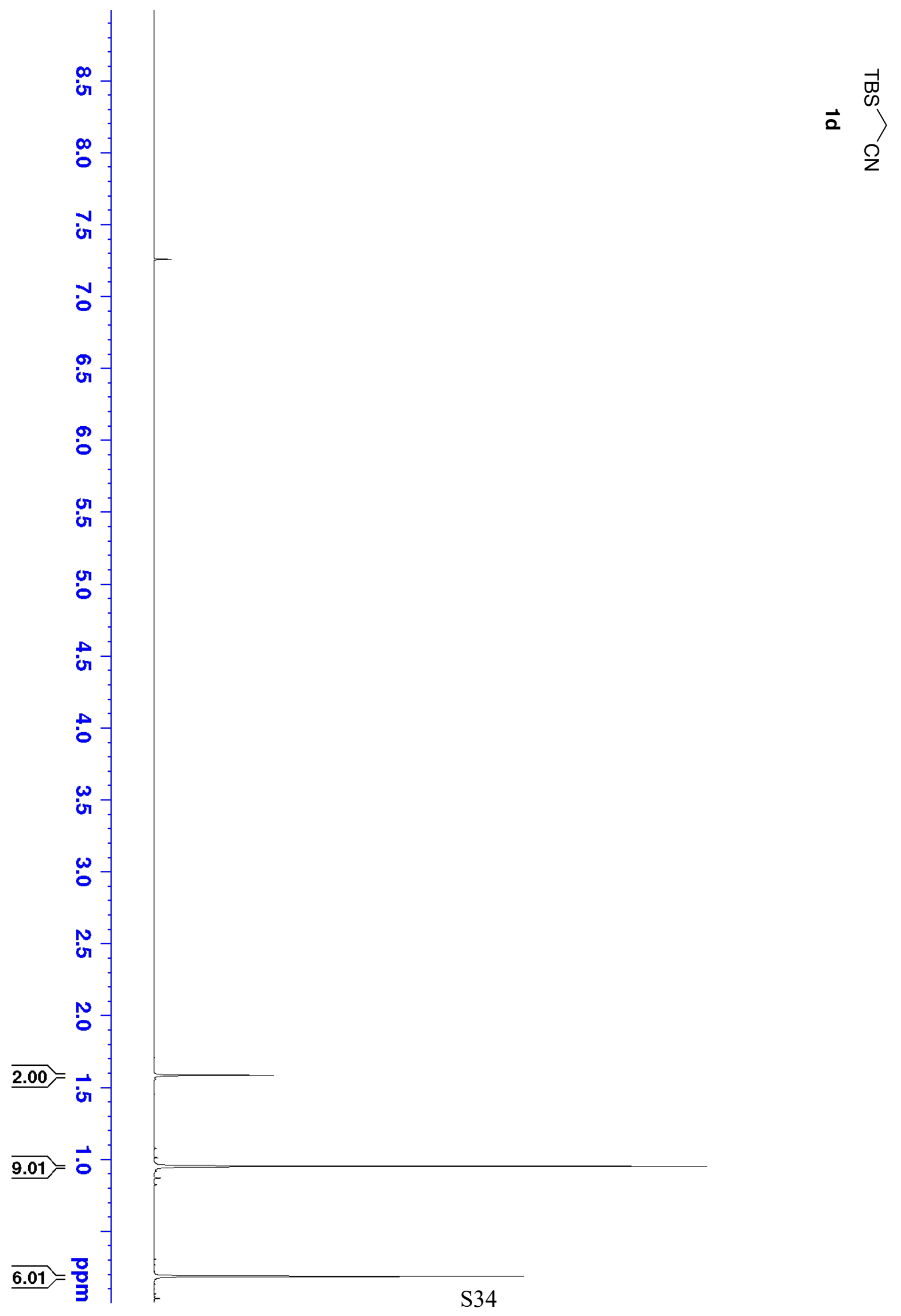




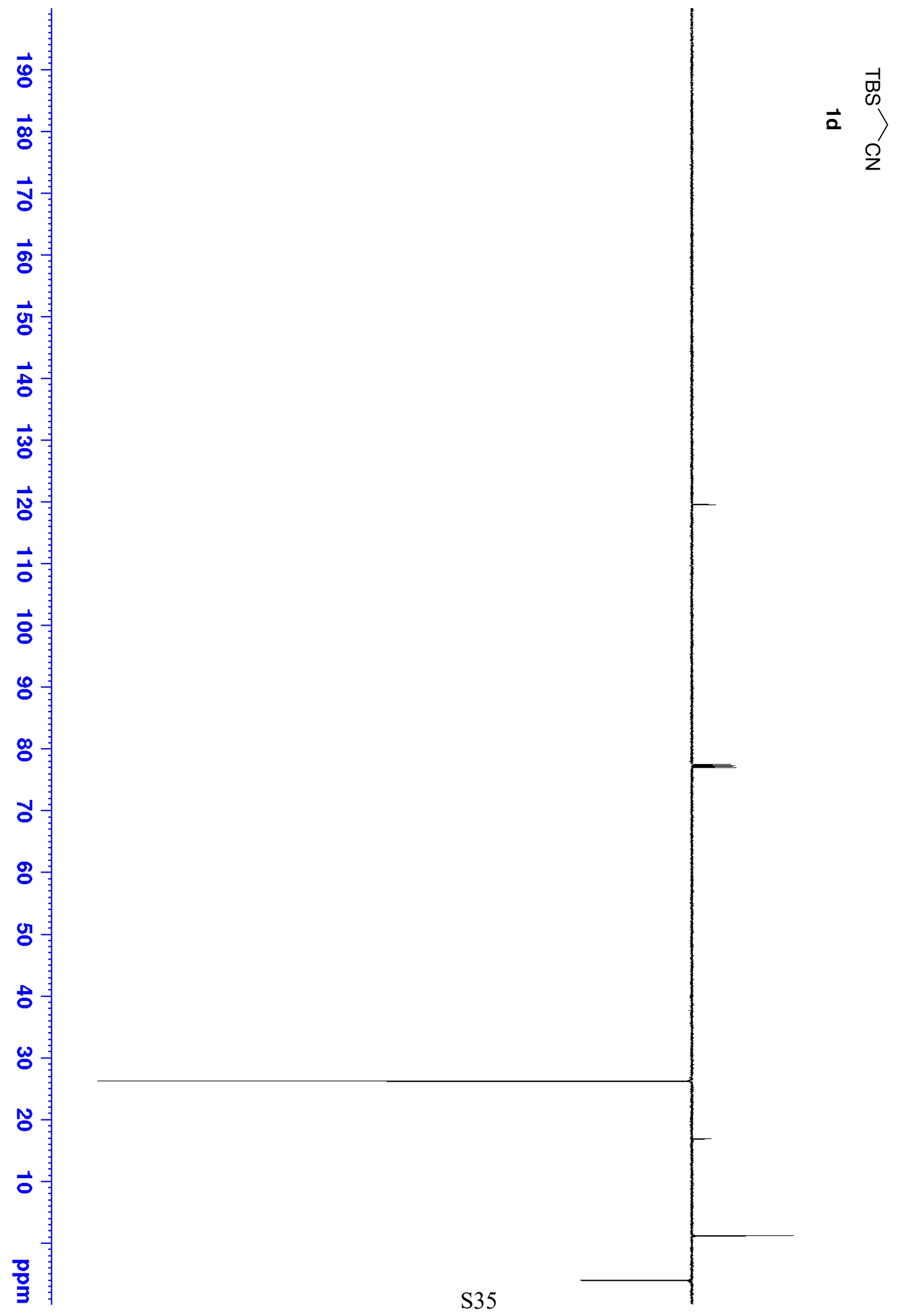




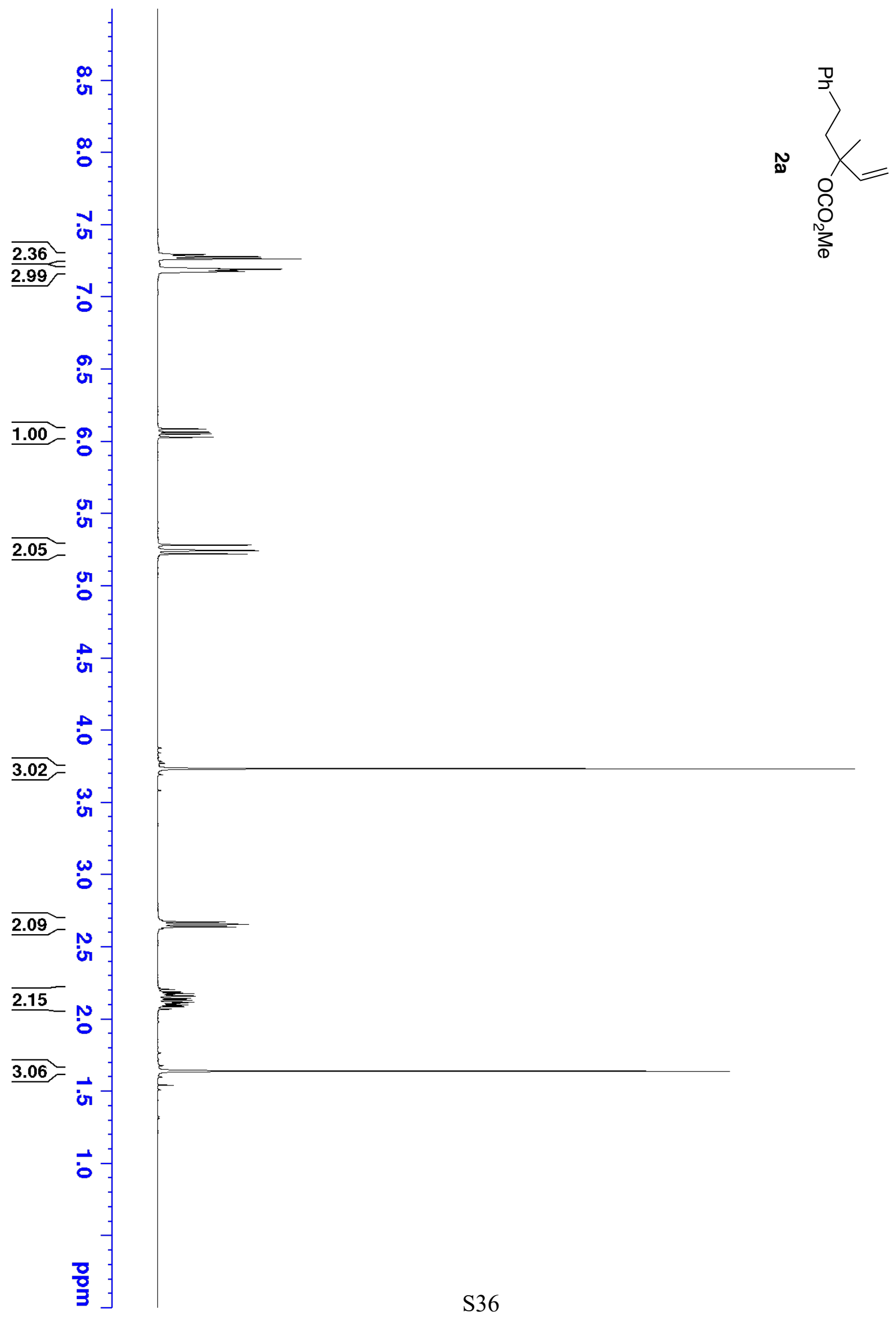




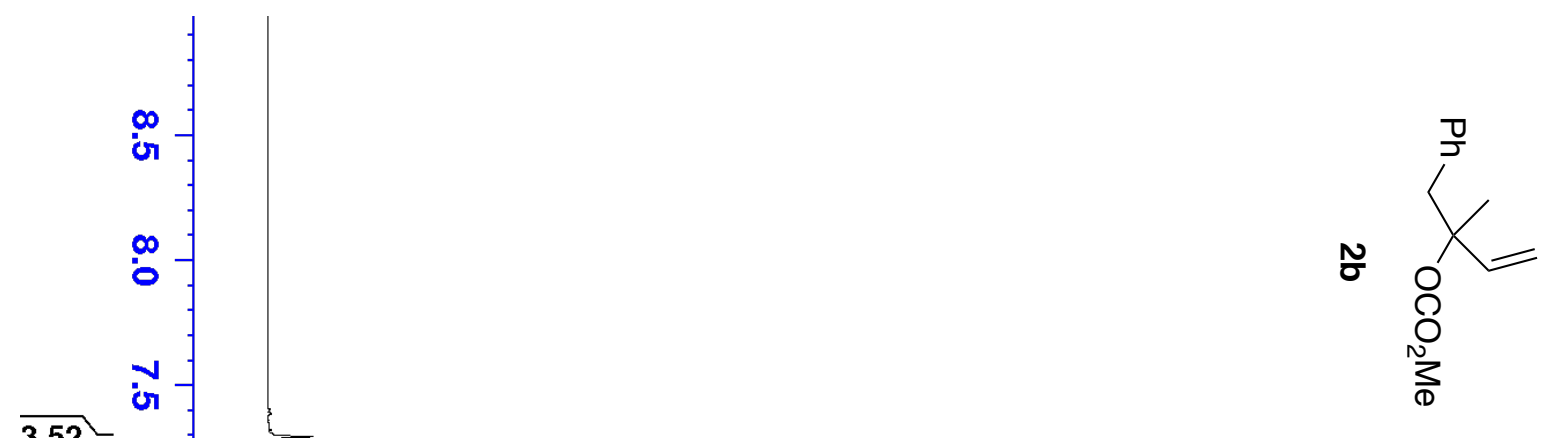




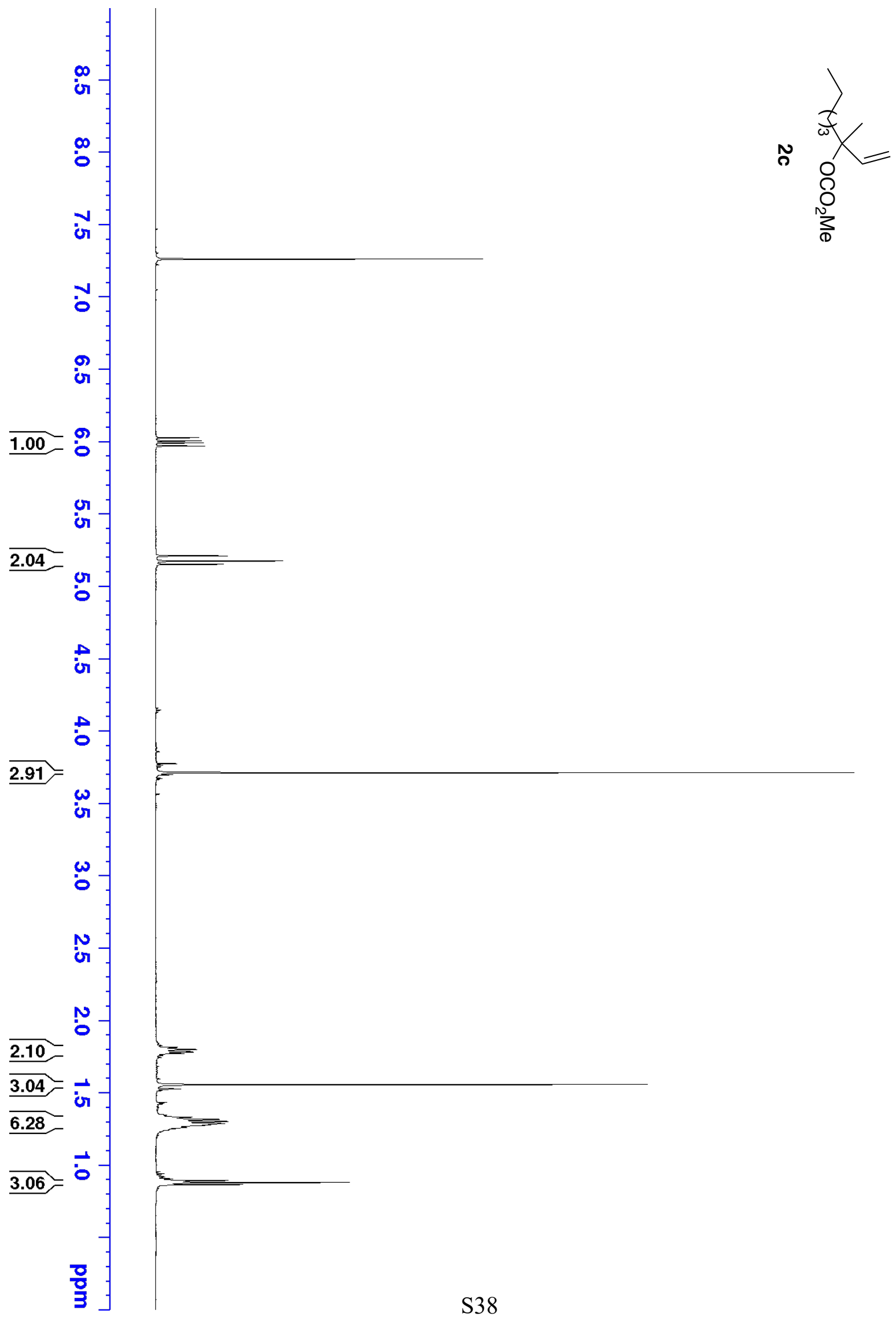



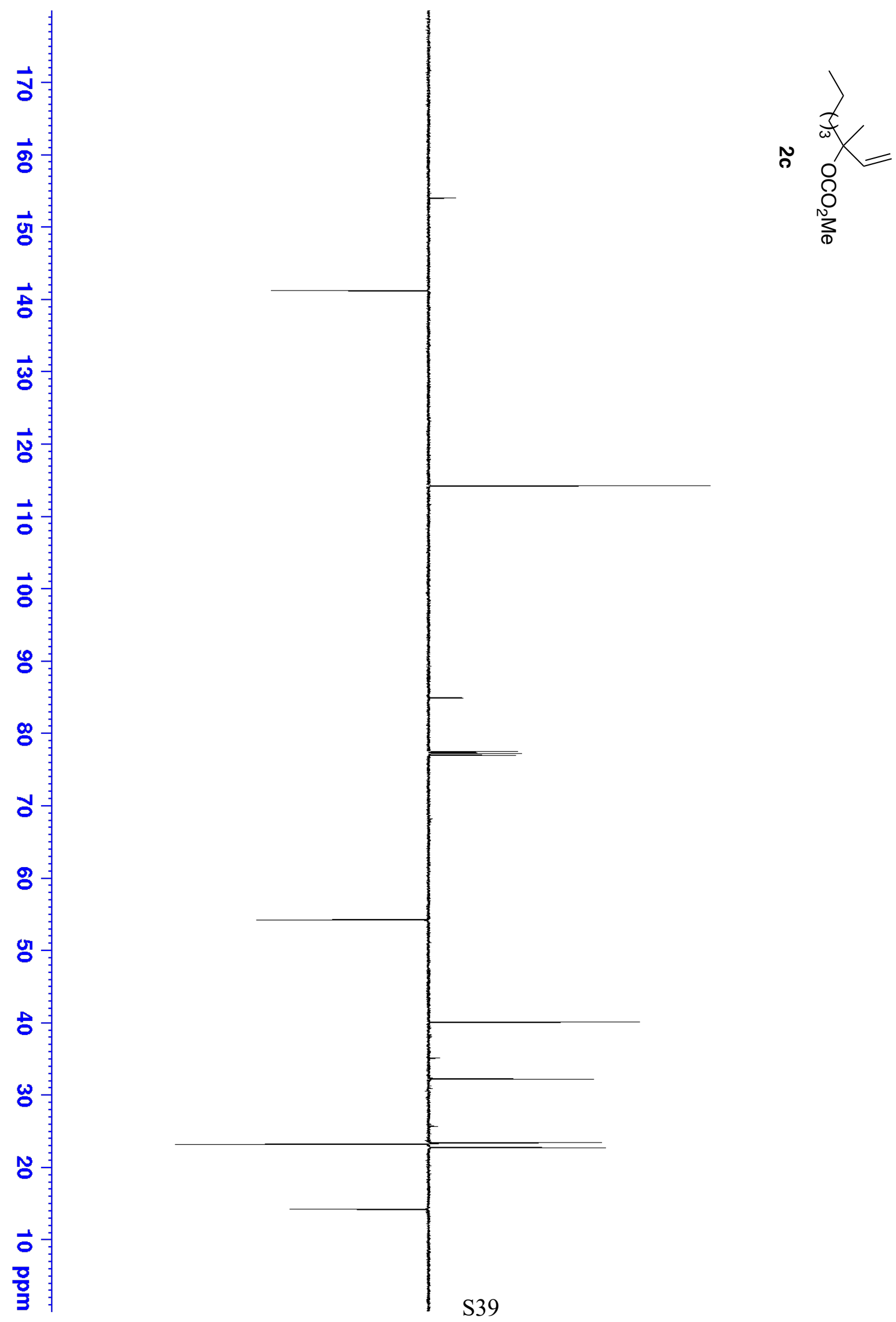


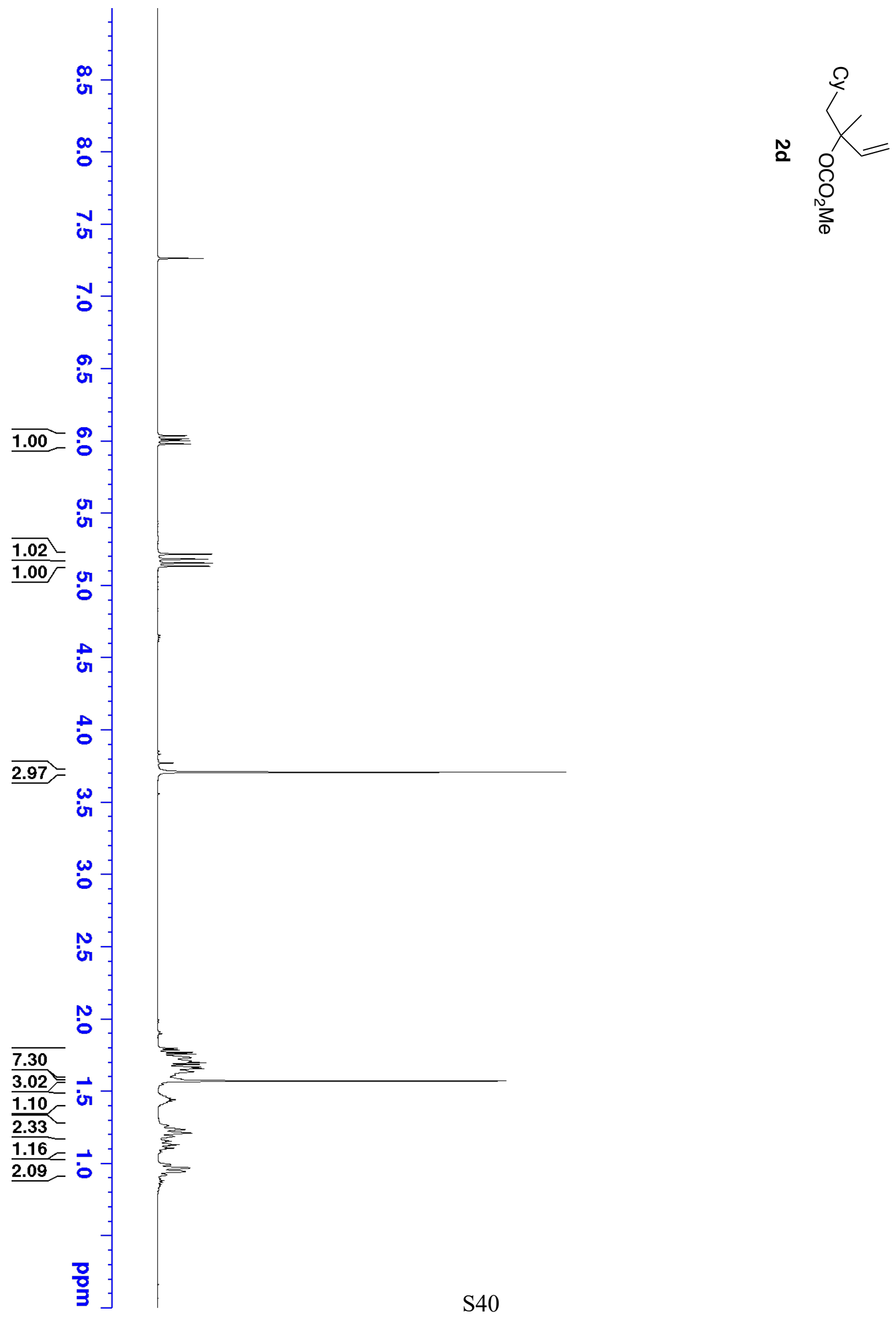




$$
\|
$$




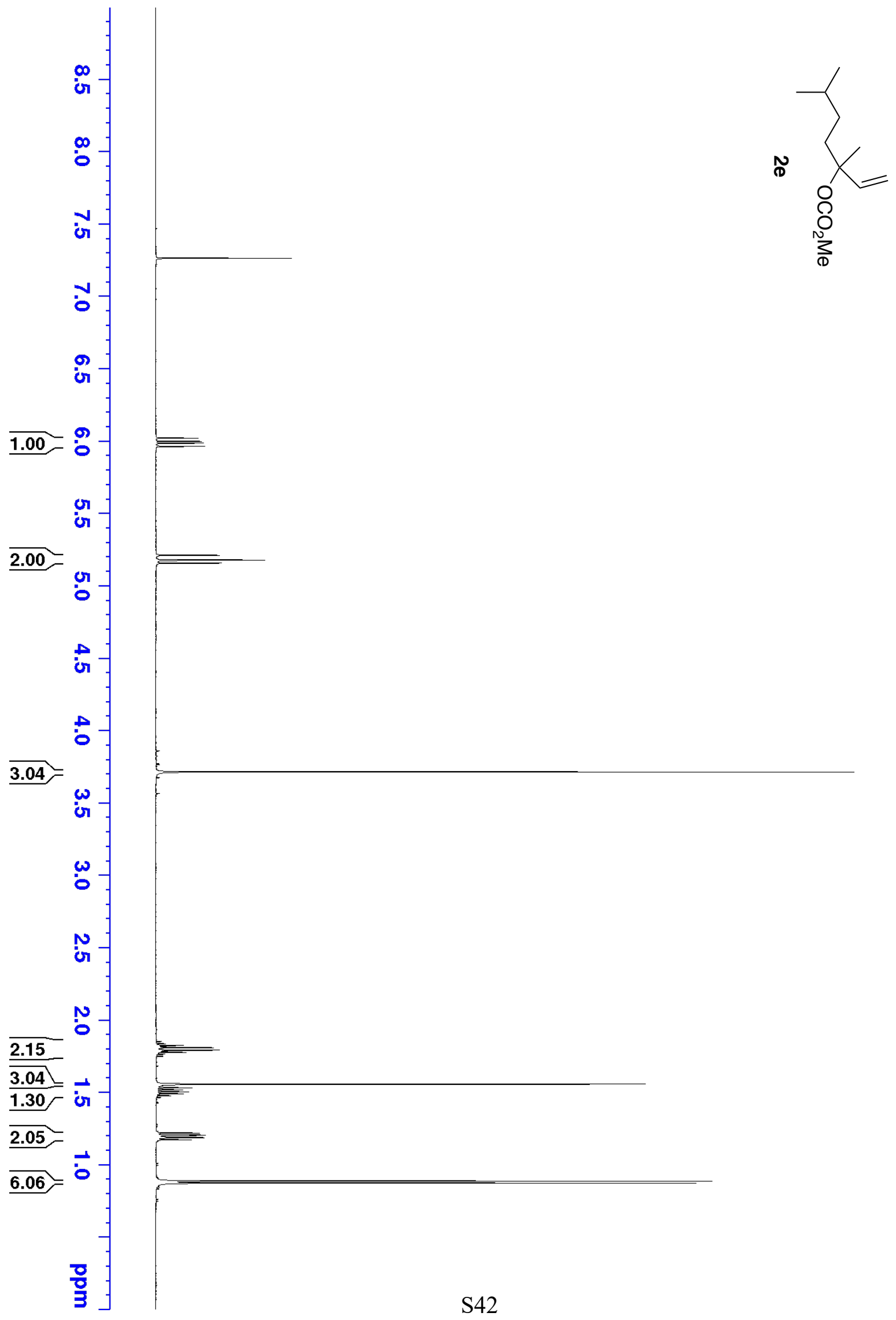




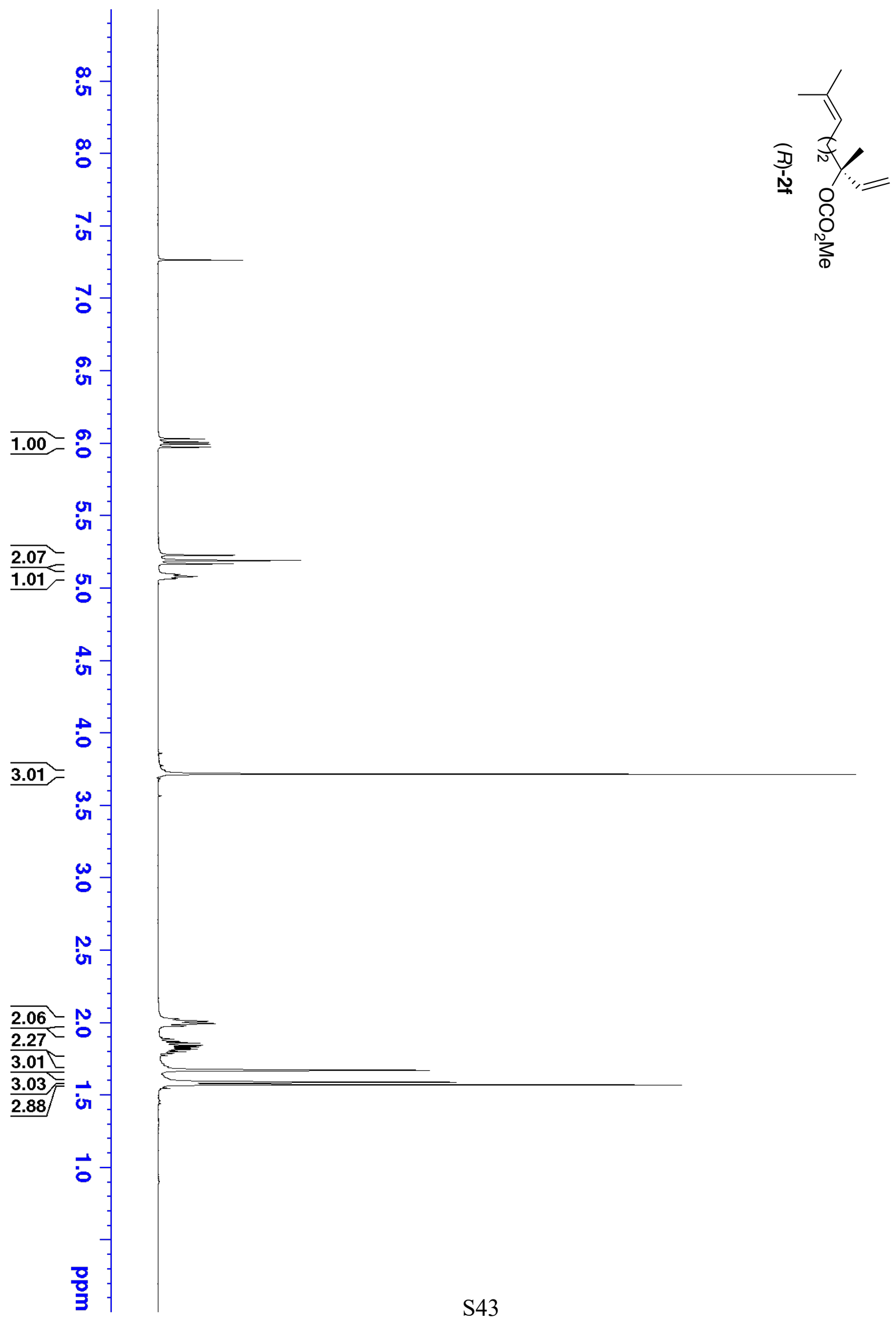




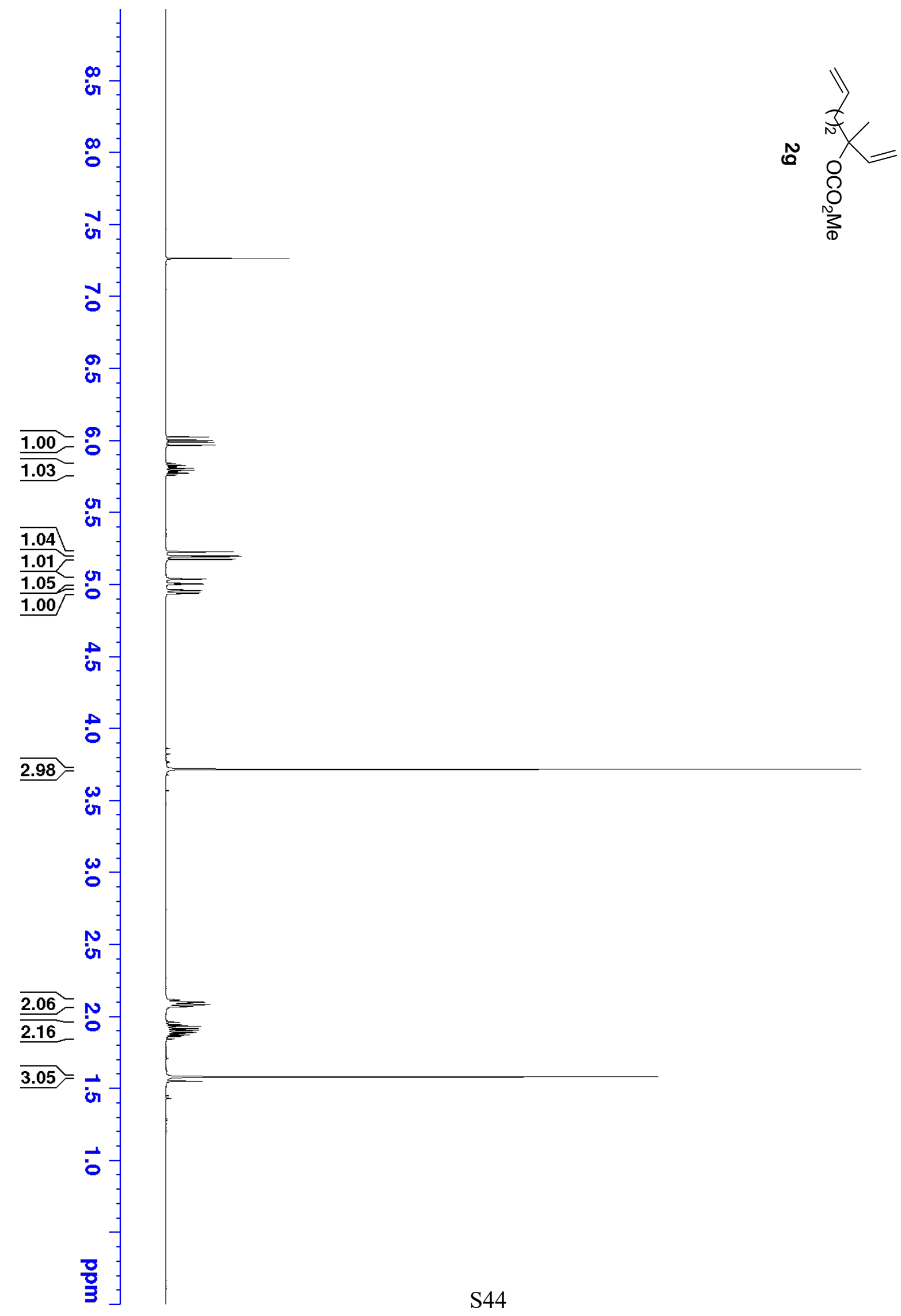




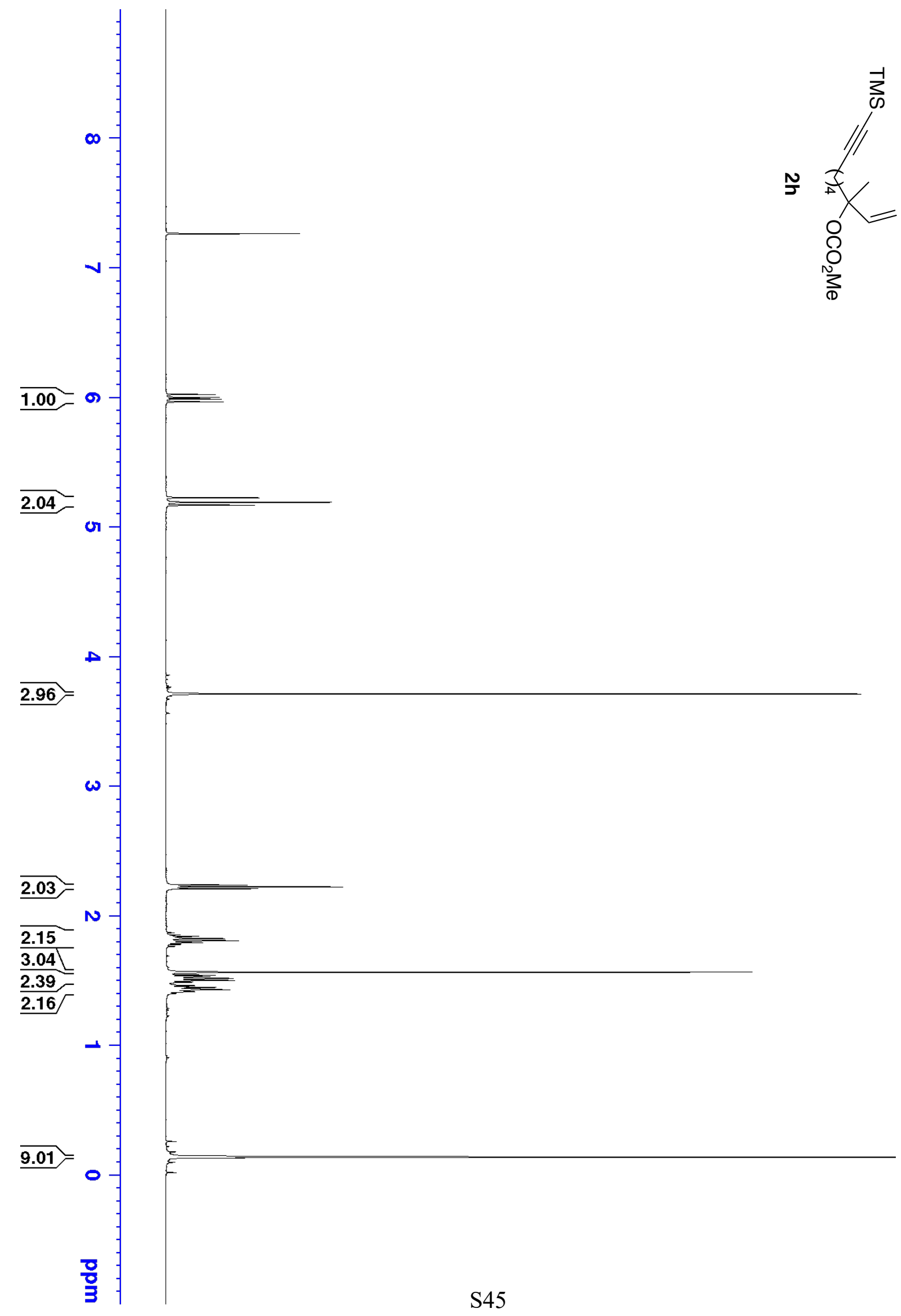




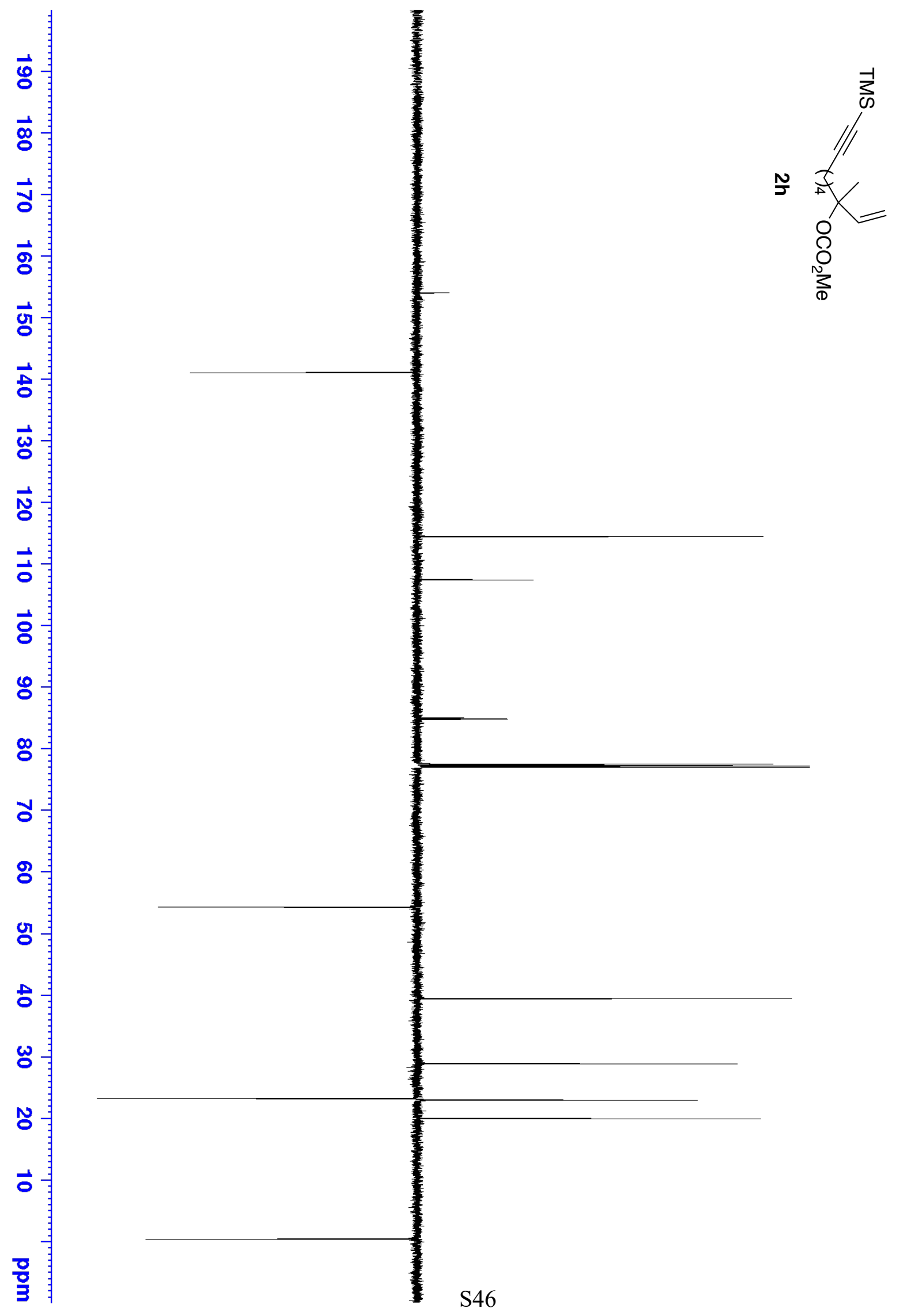




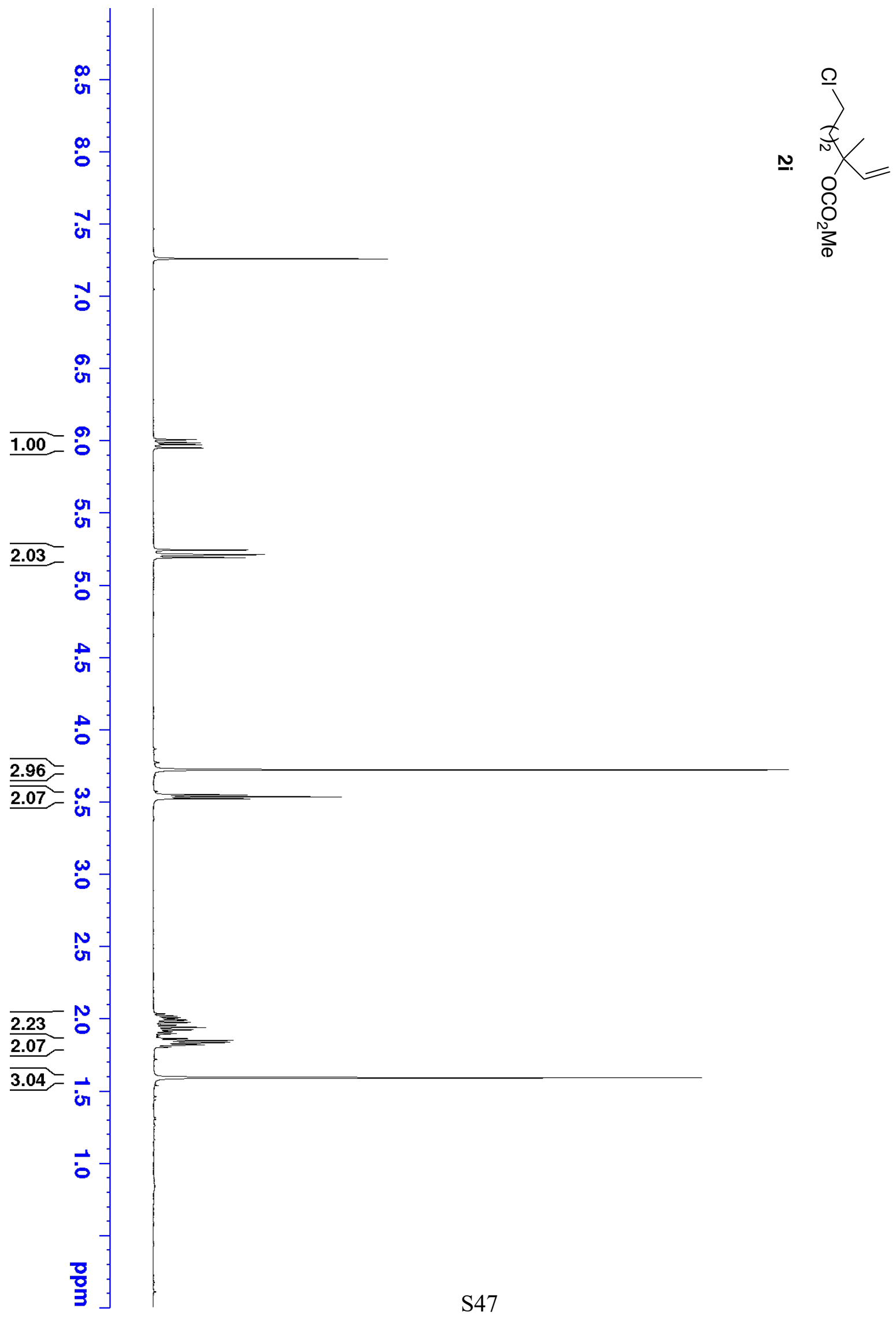




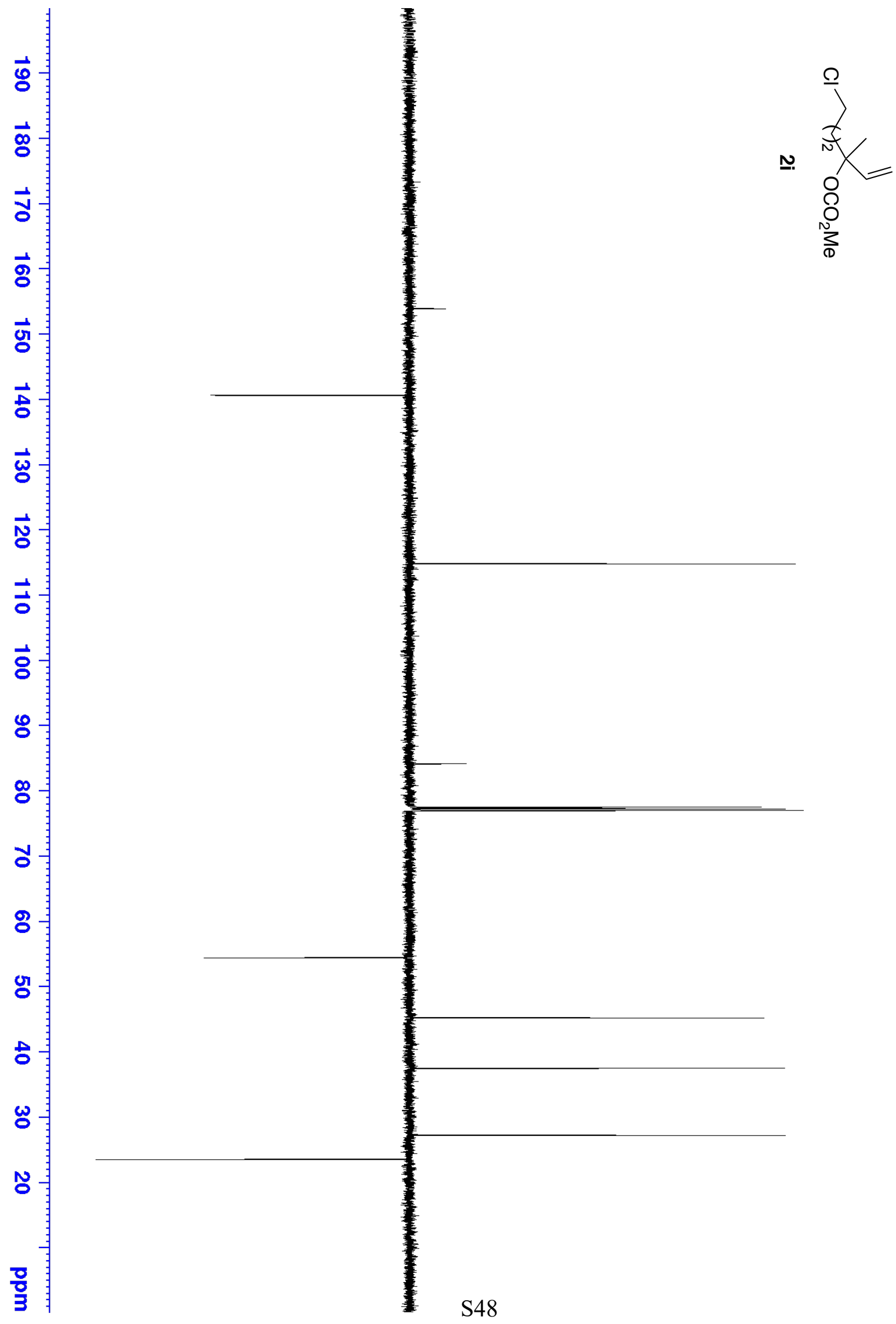




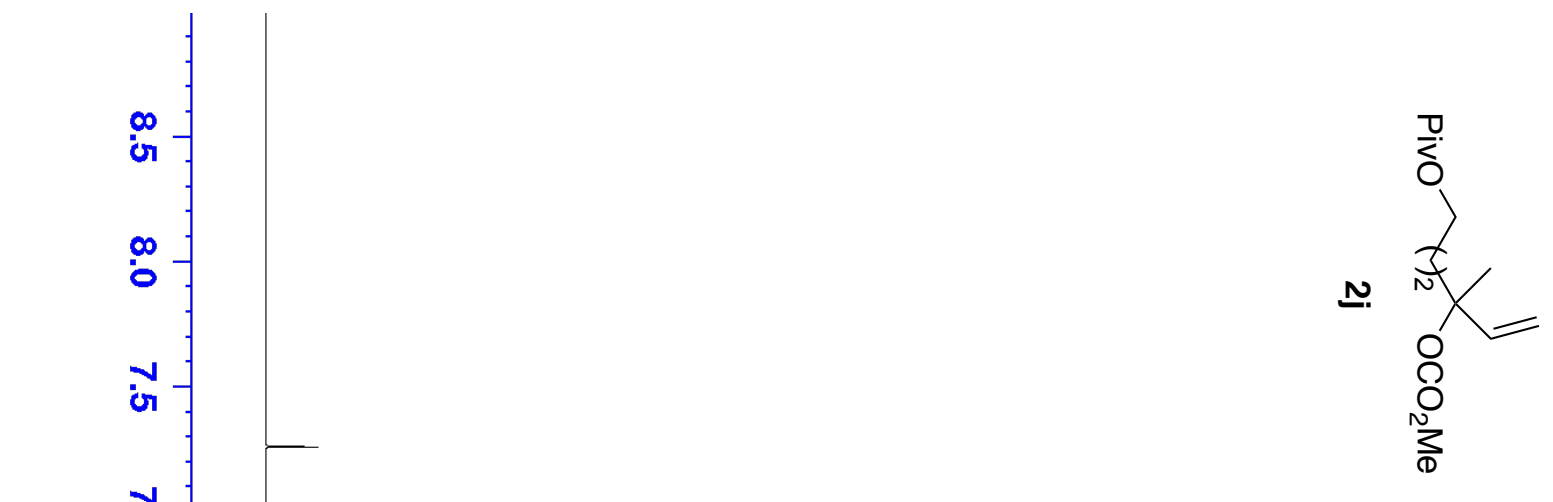




$$
||^{\prime}
$$




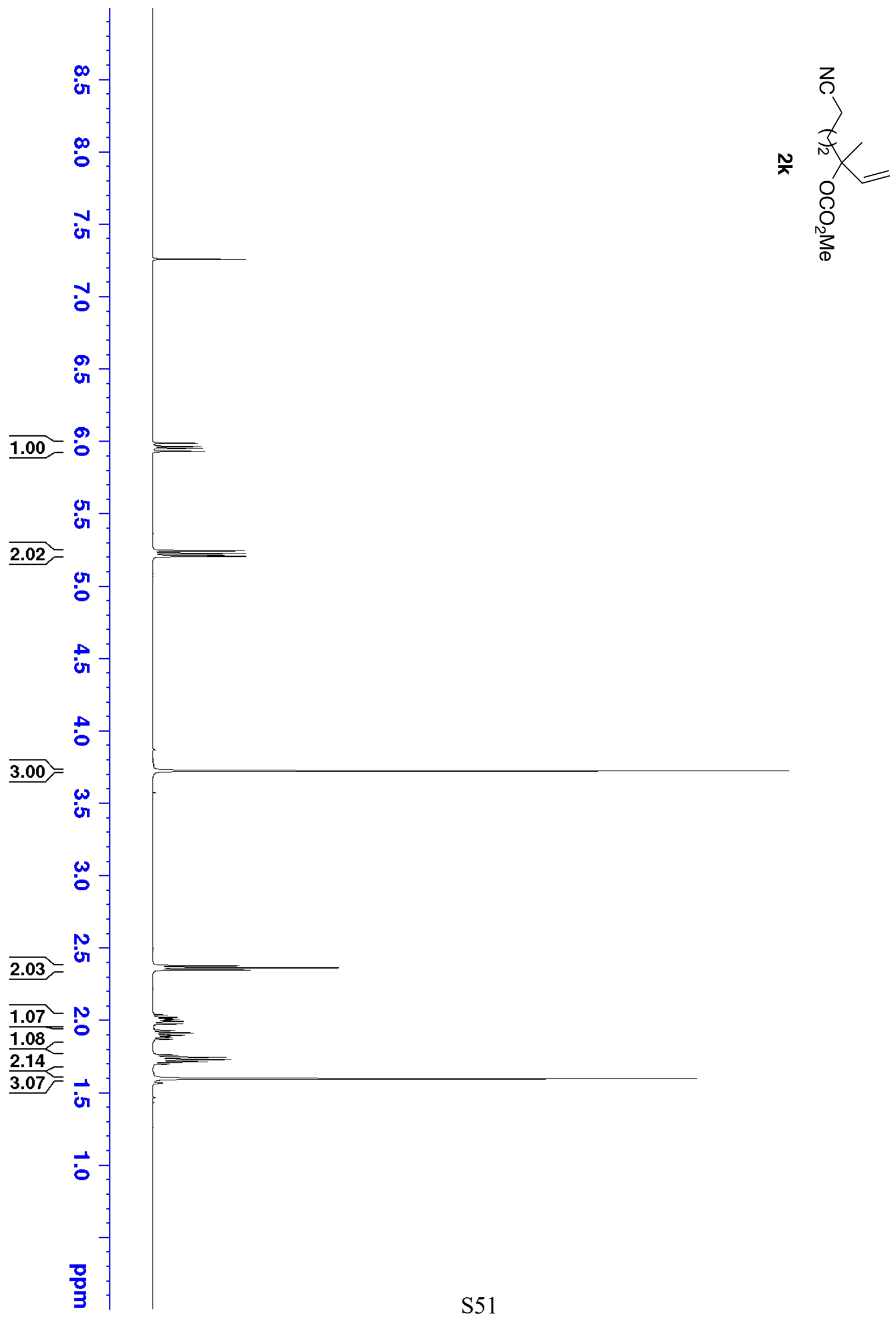




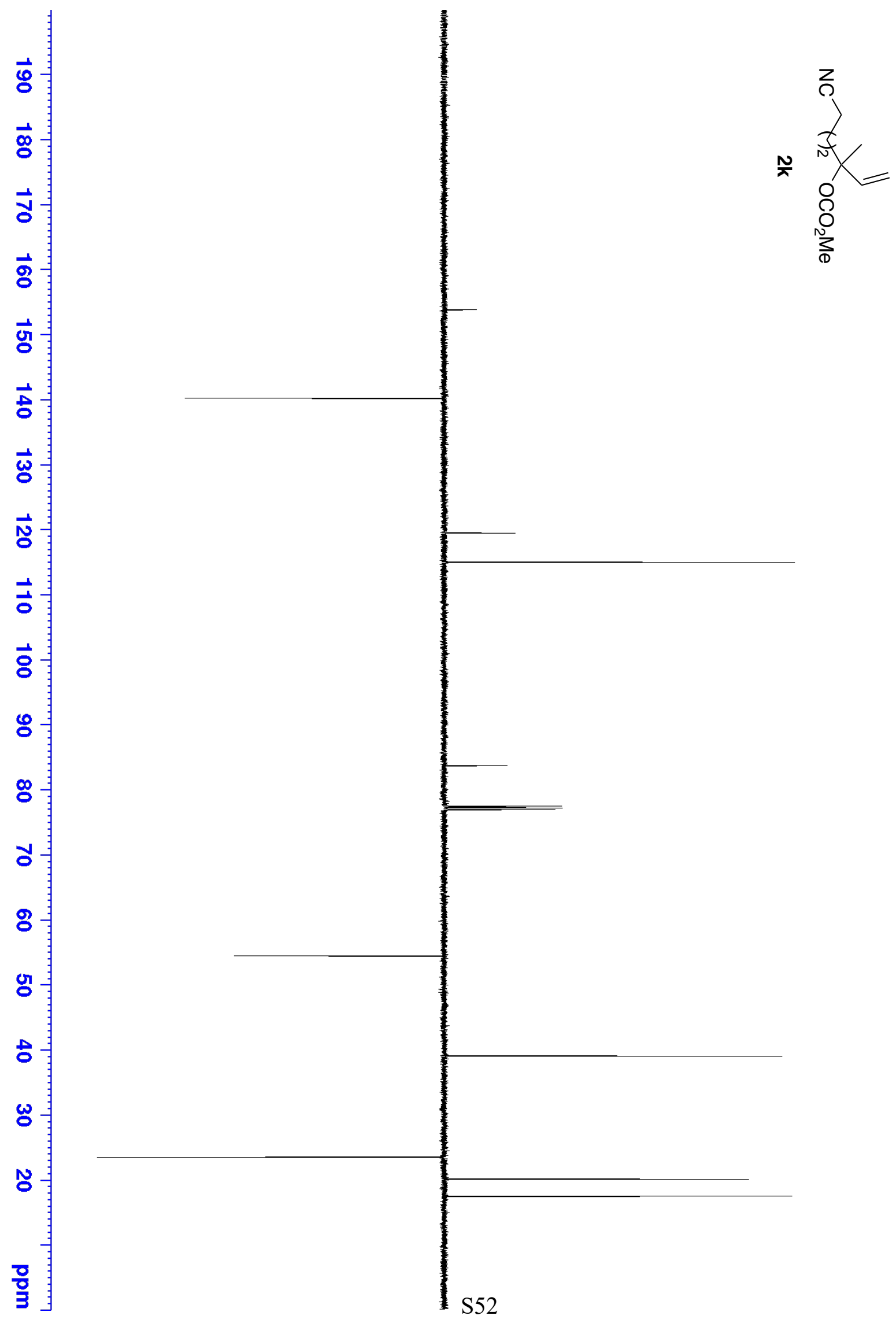




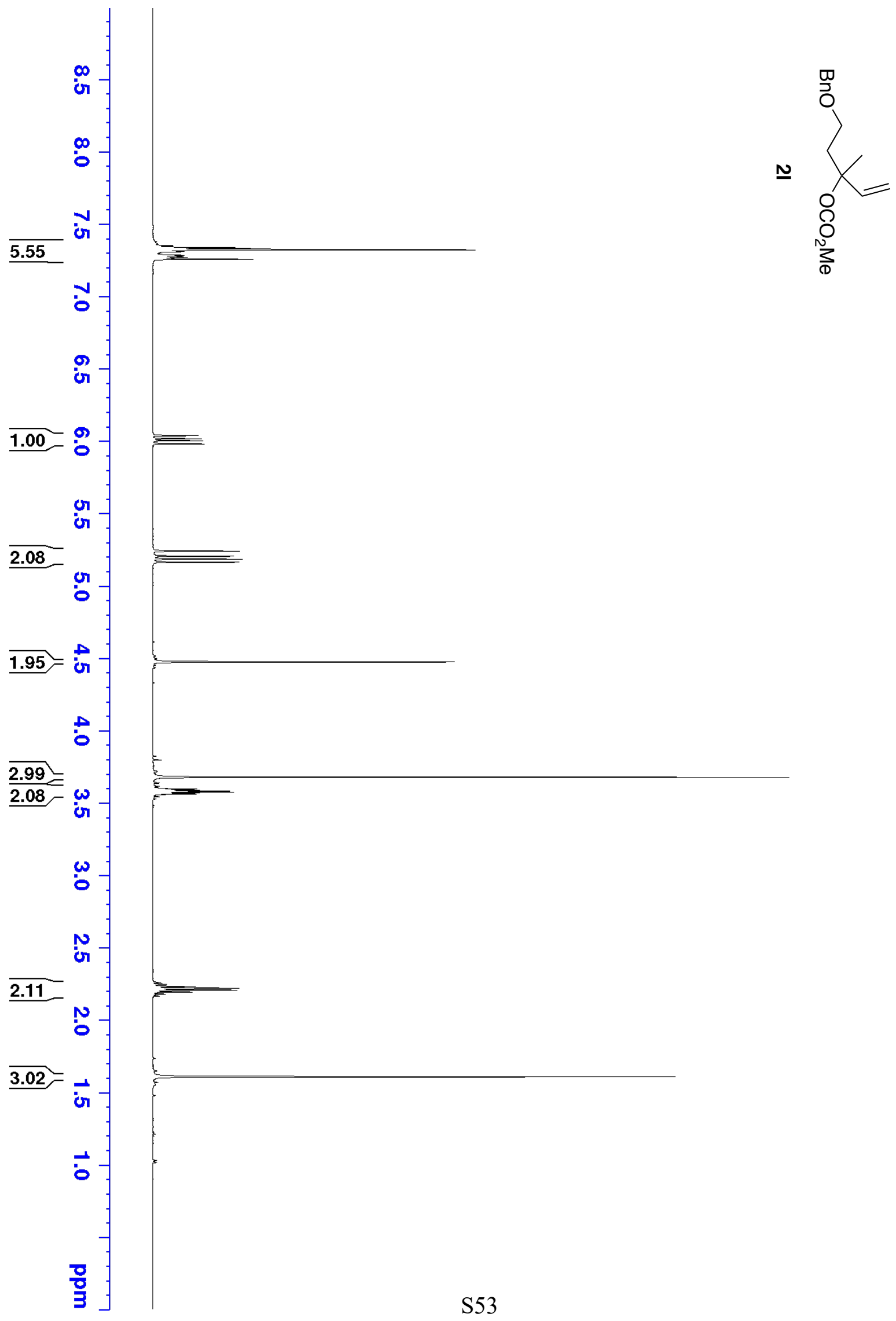




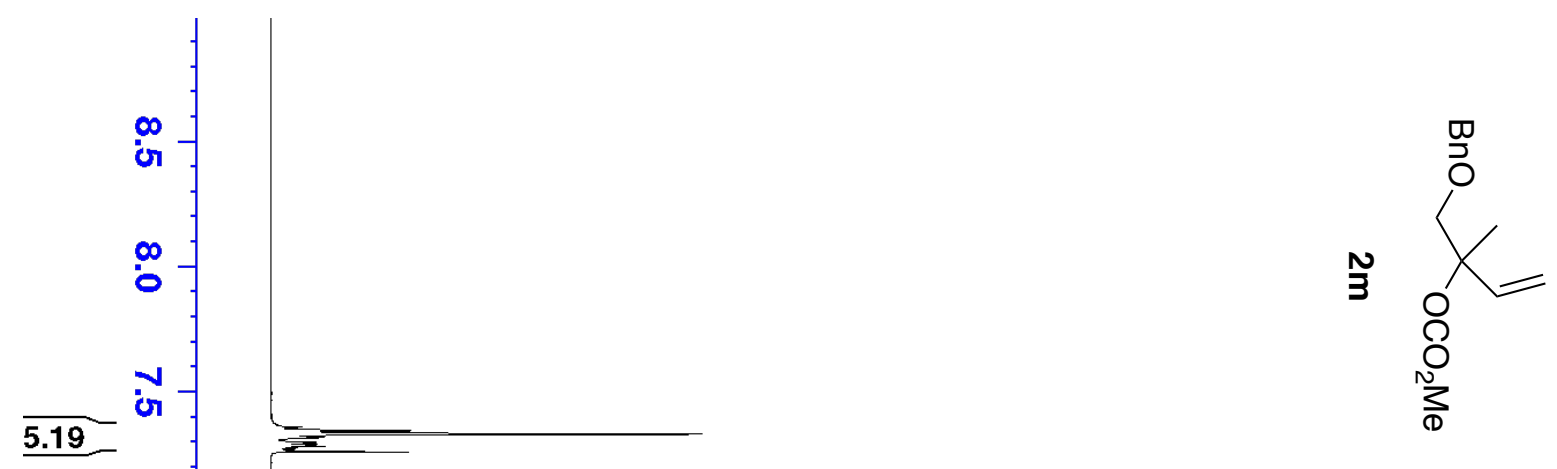



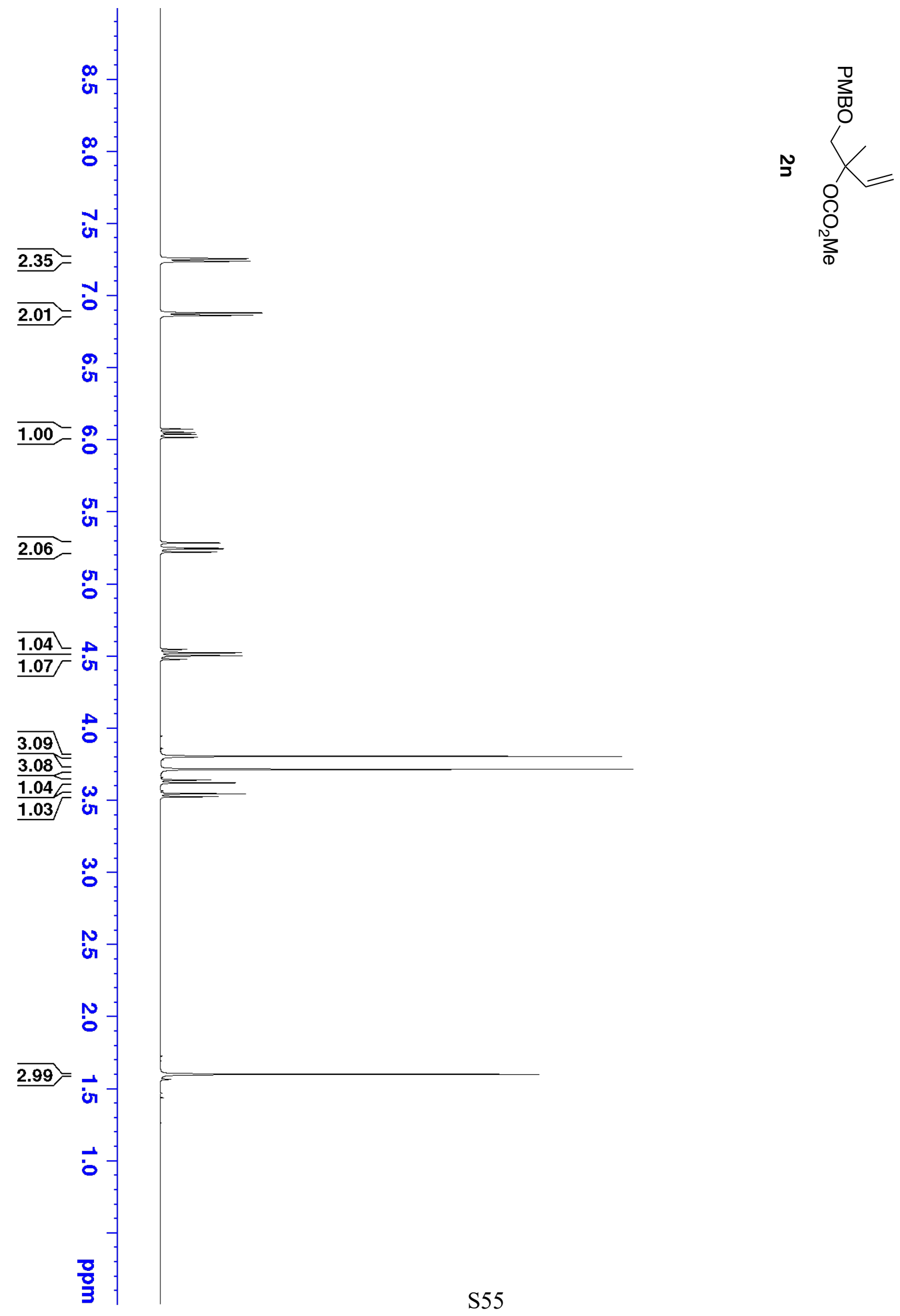


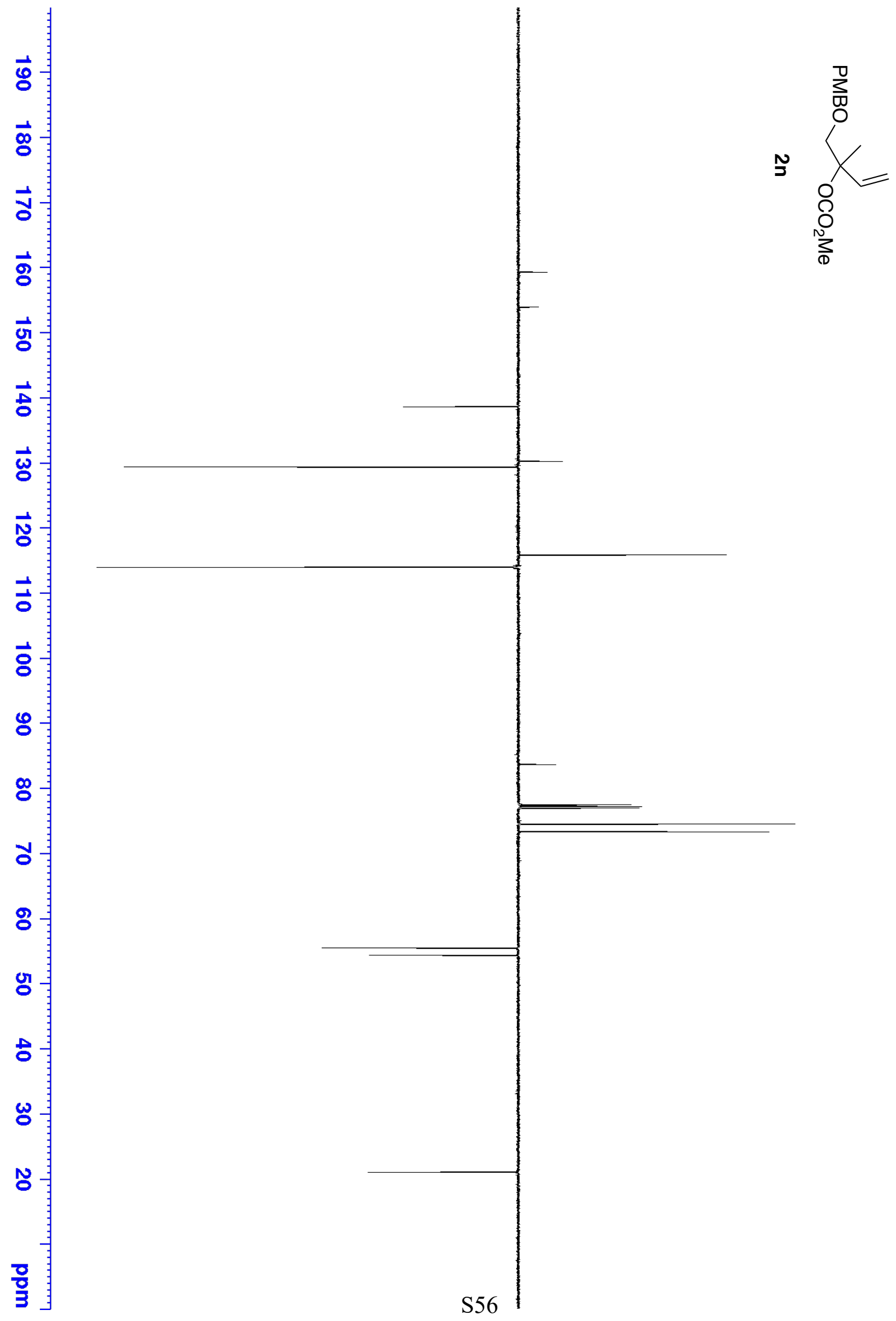




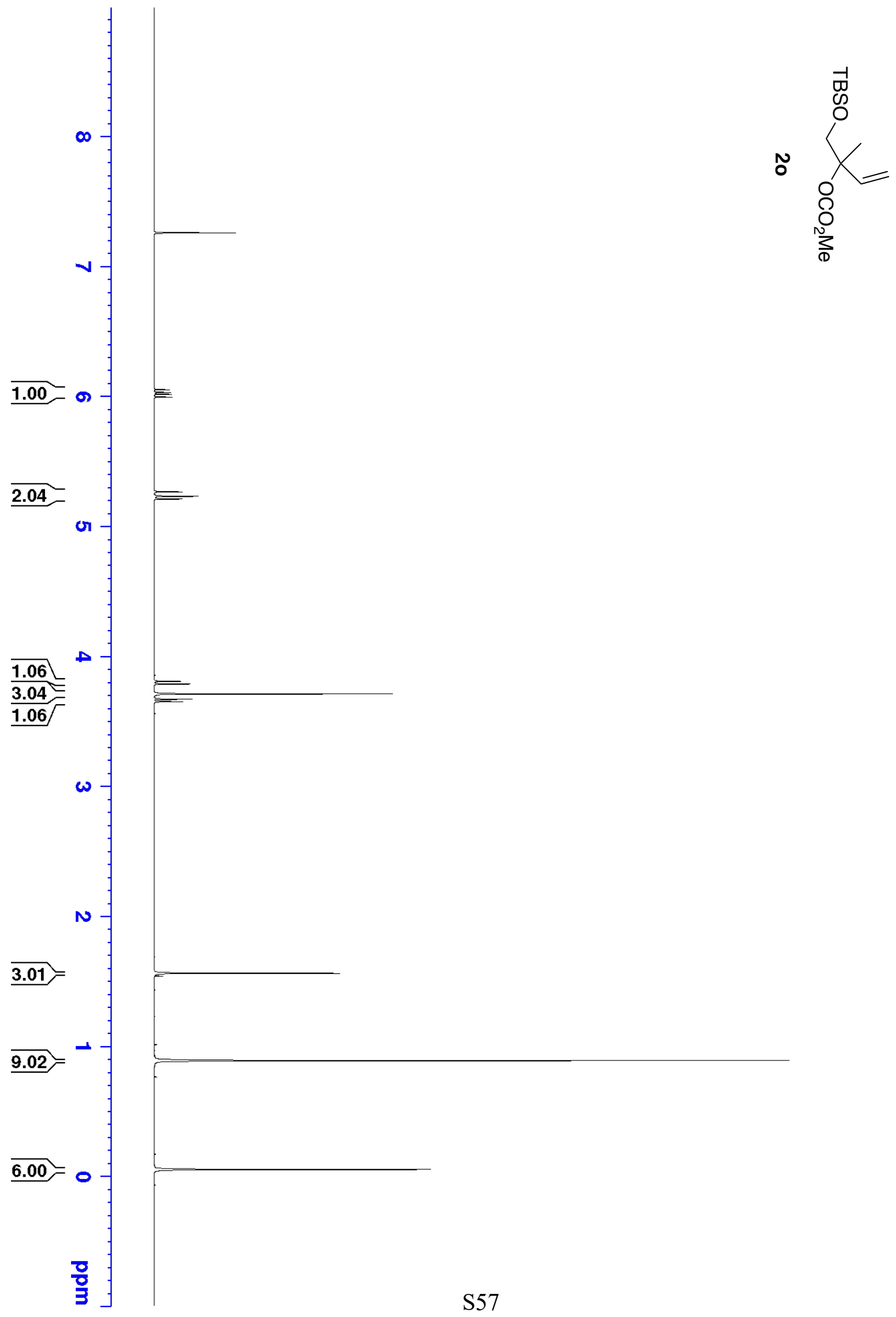




$$
\text { |f }
$$




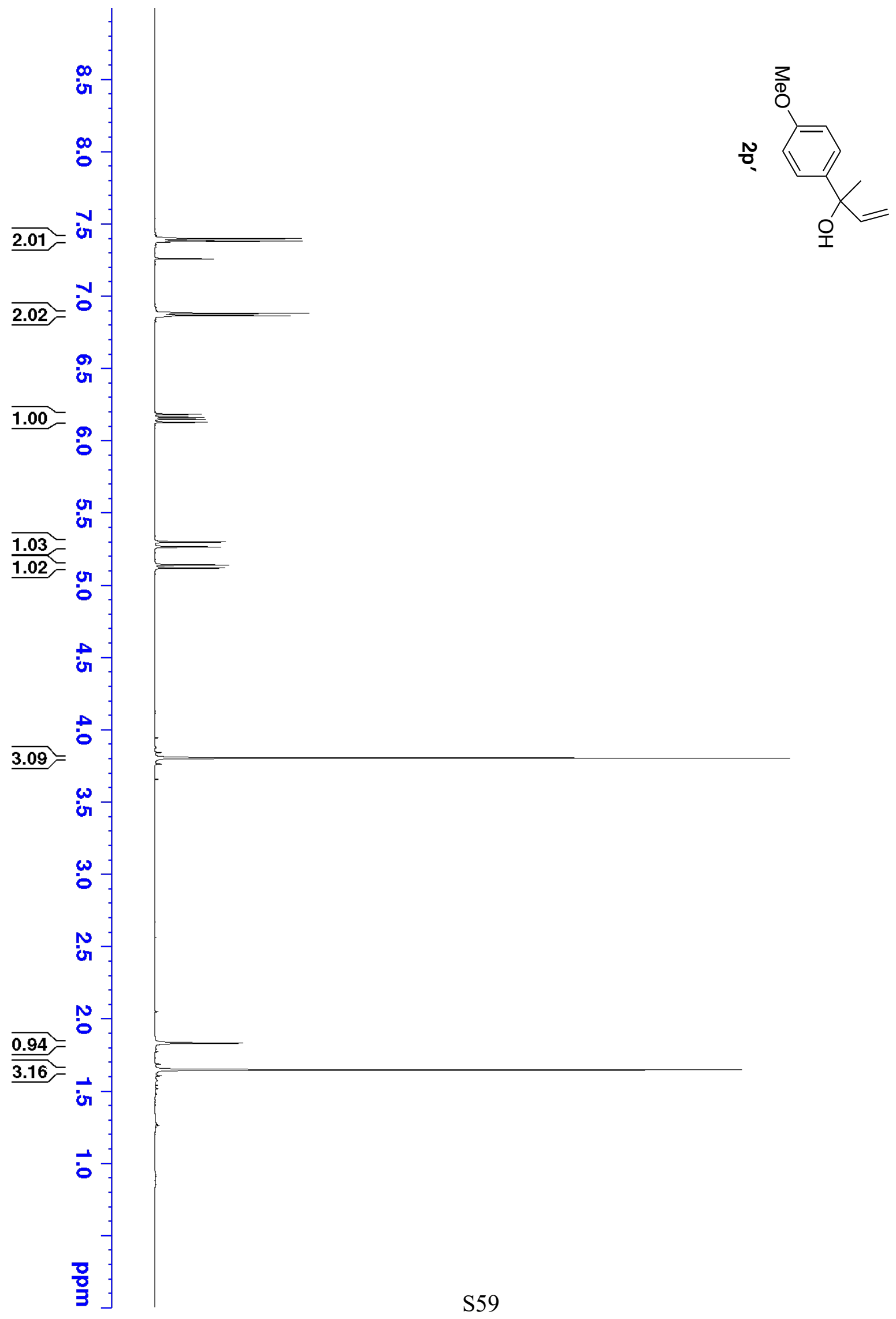




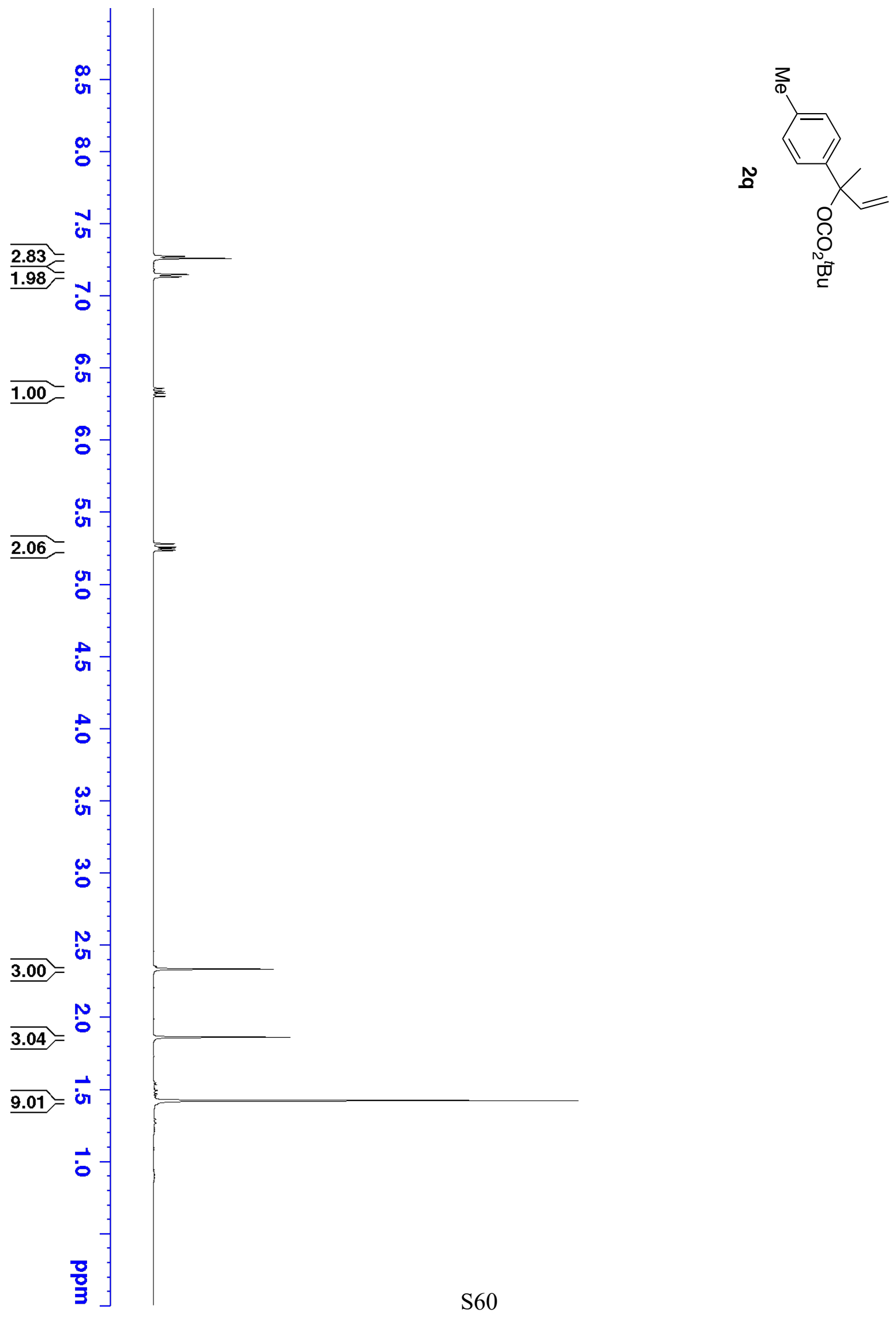



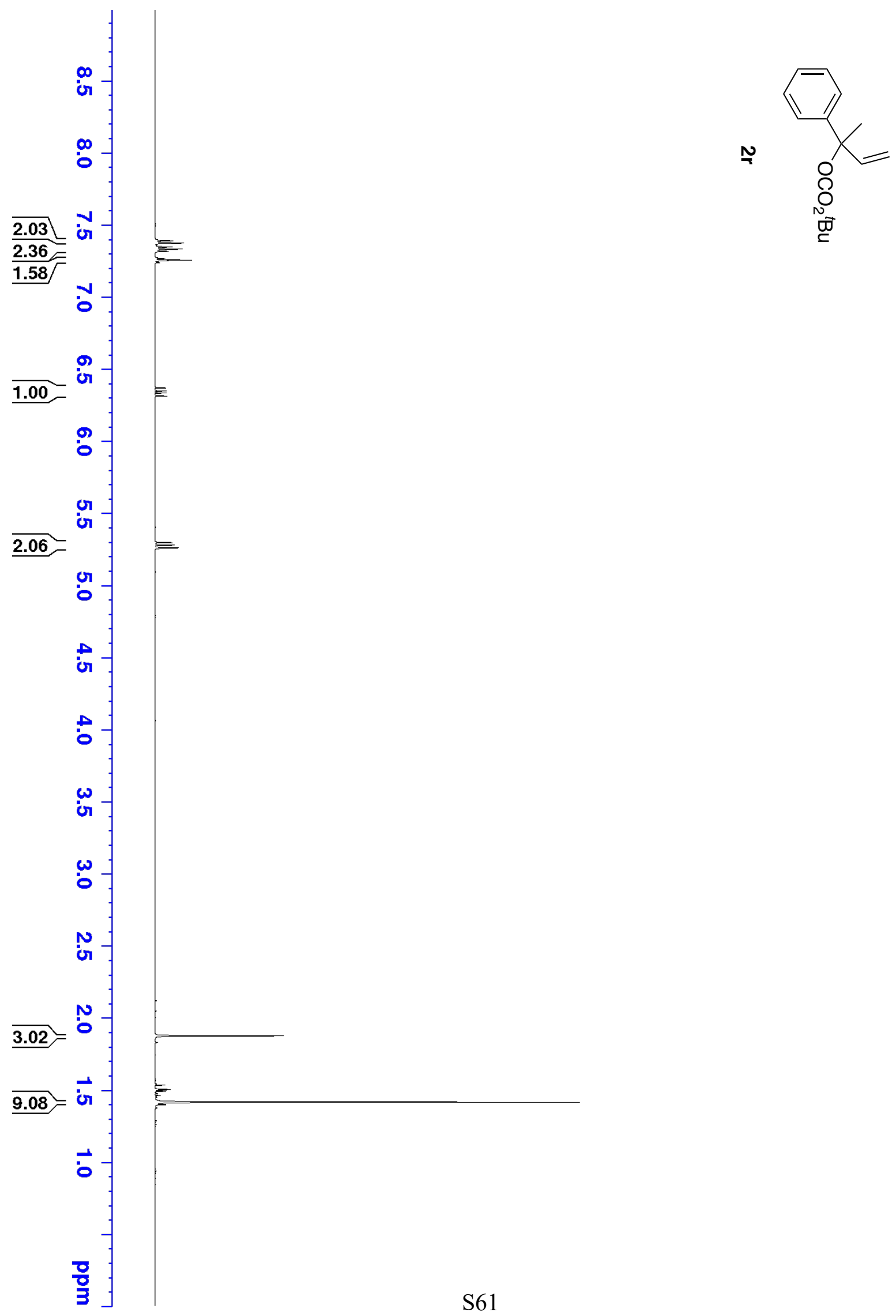


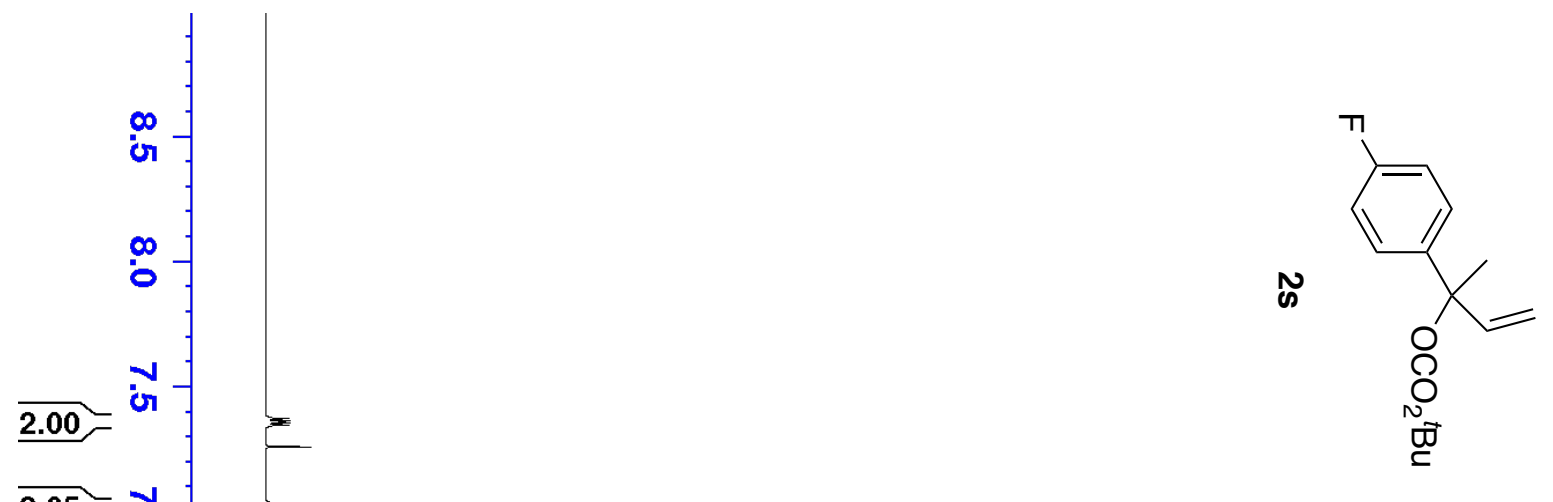




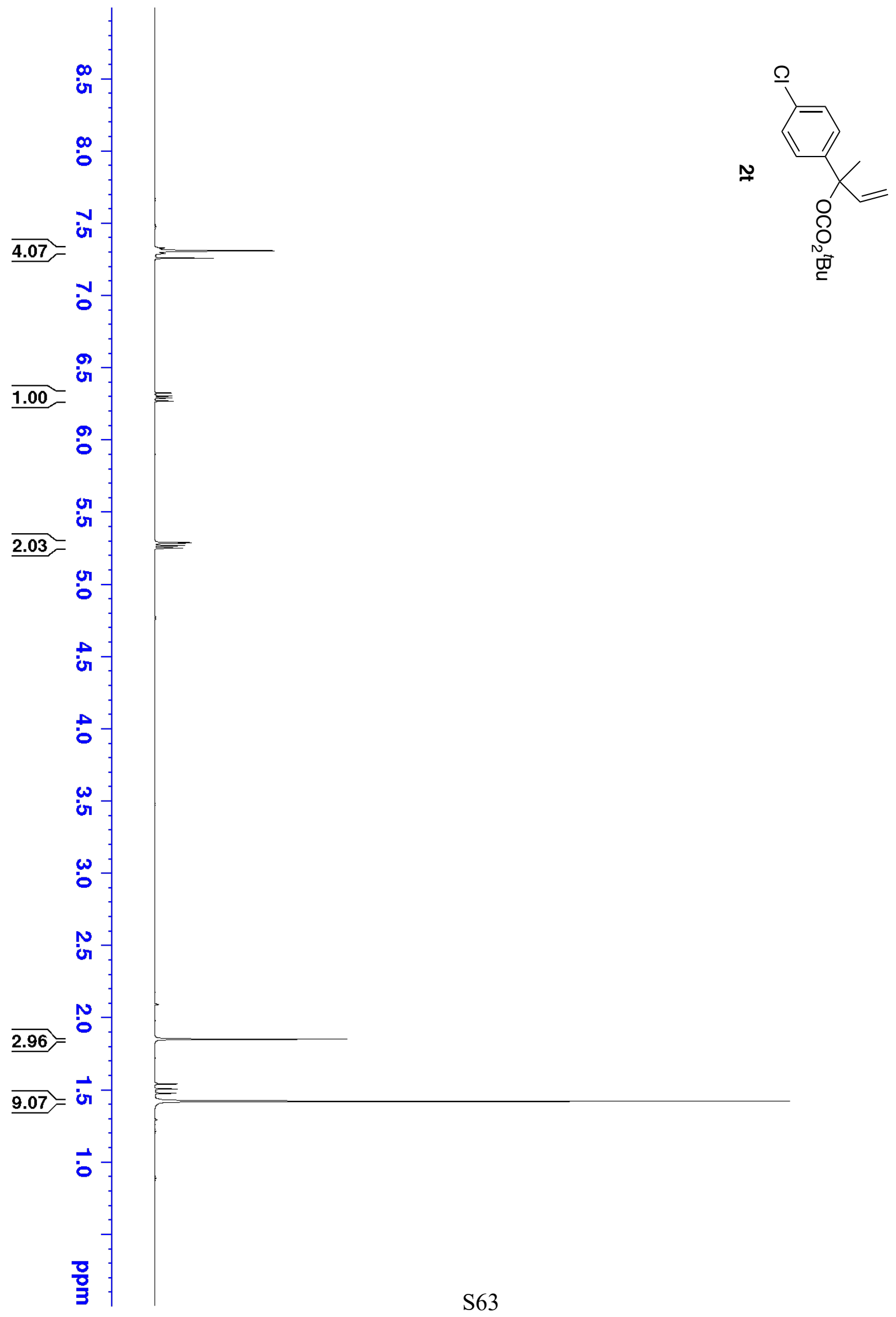




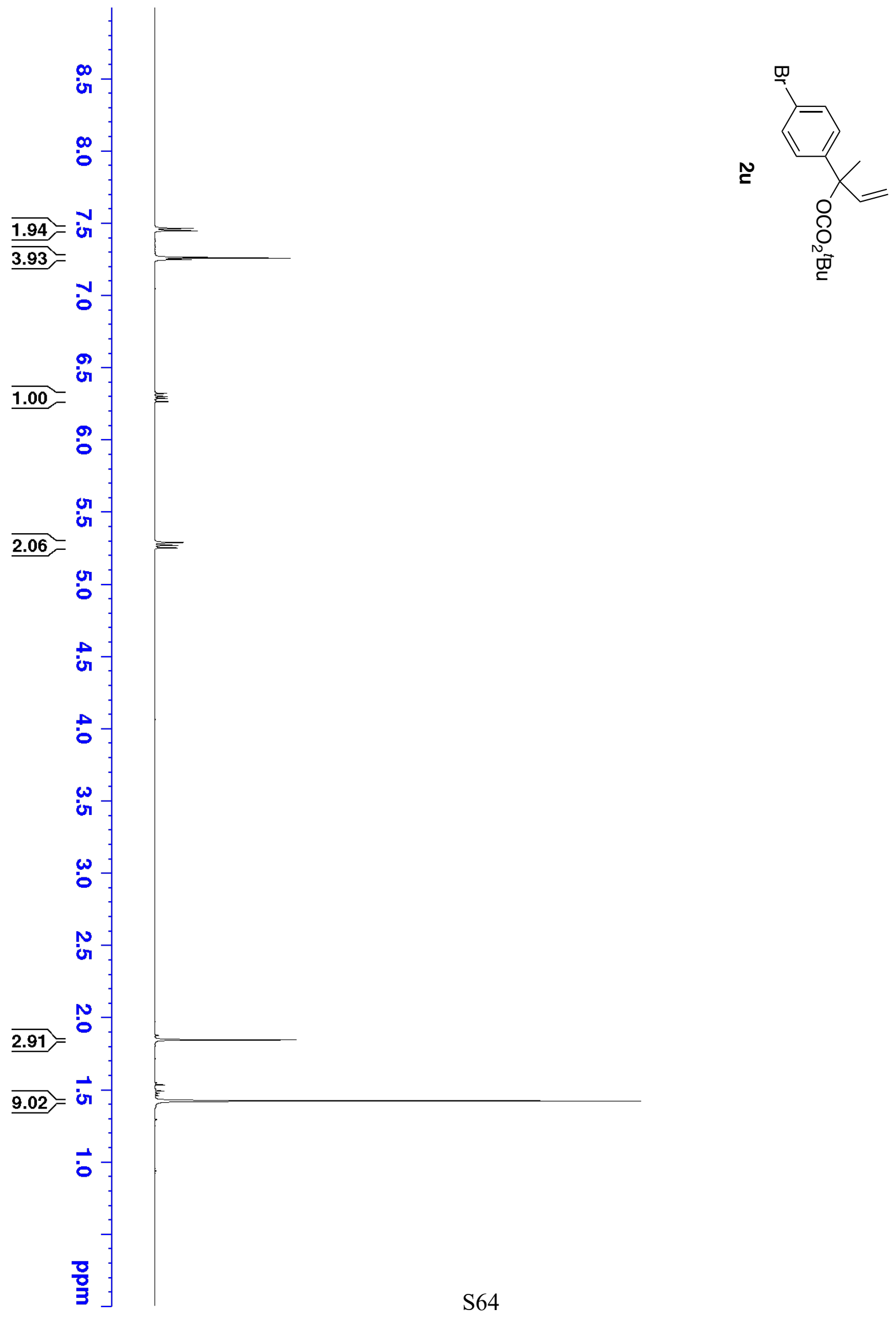




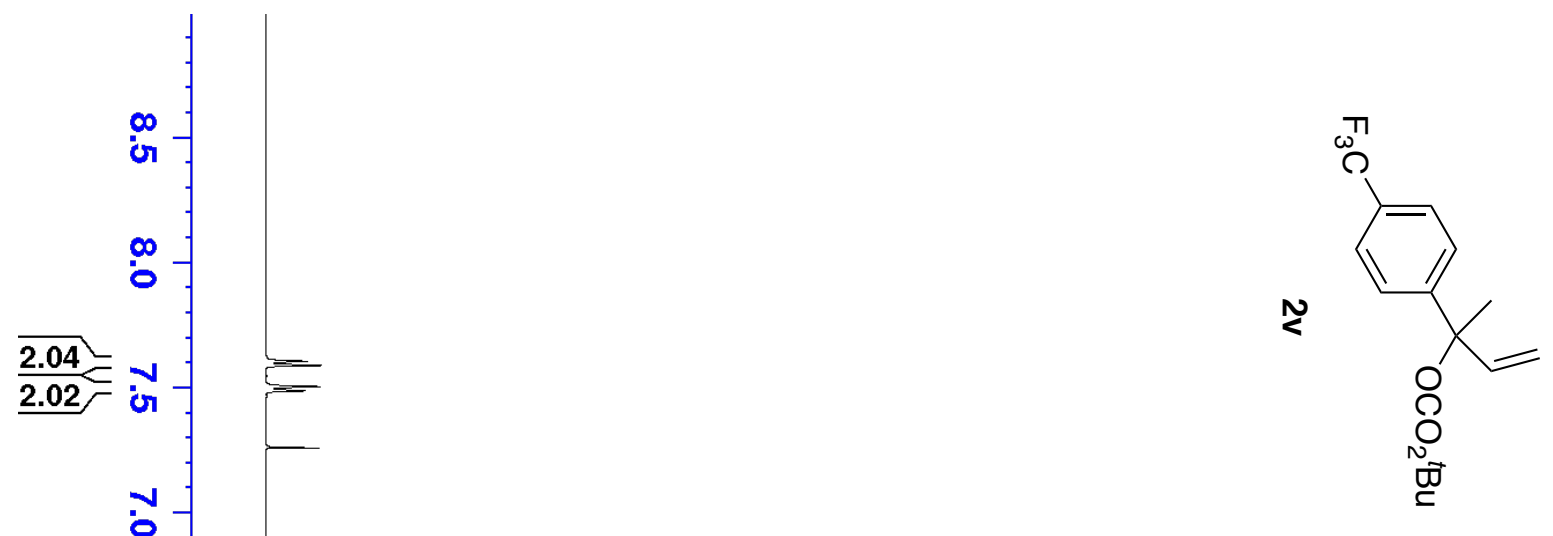




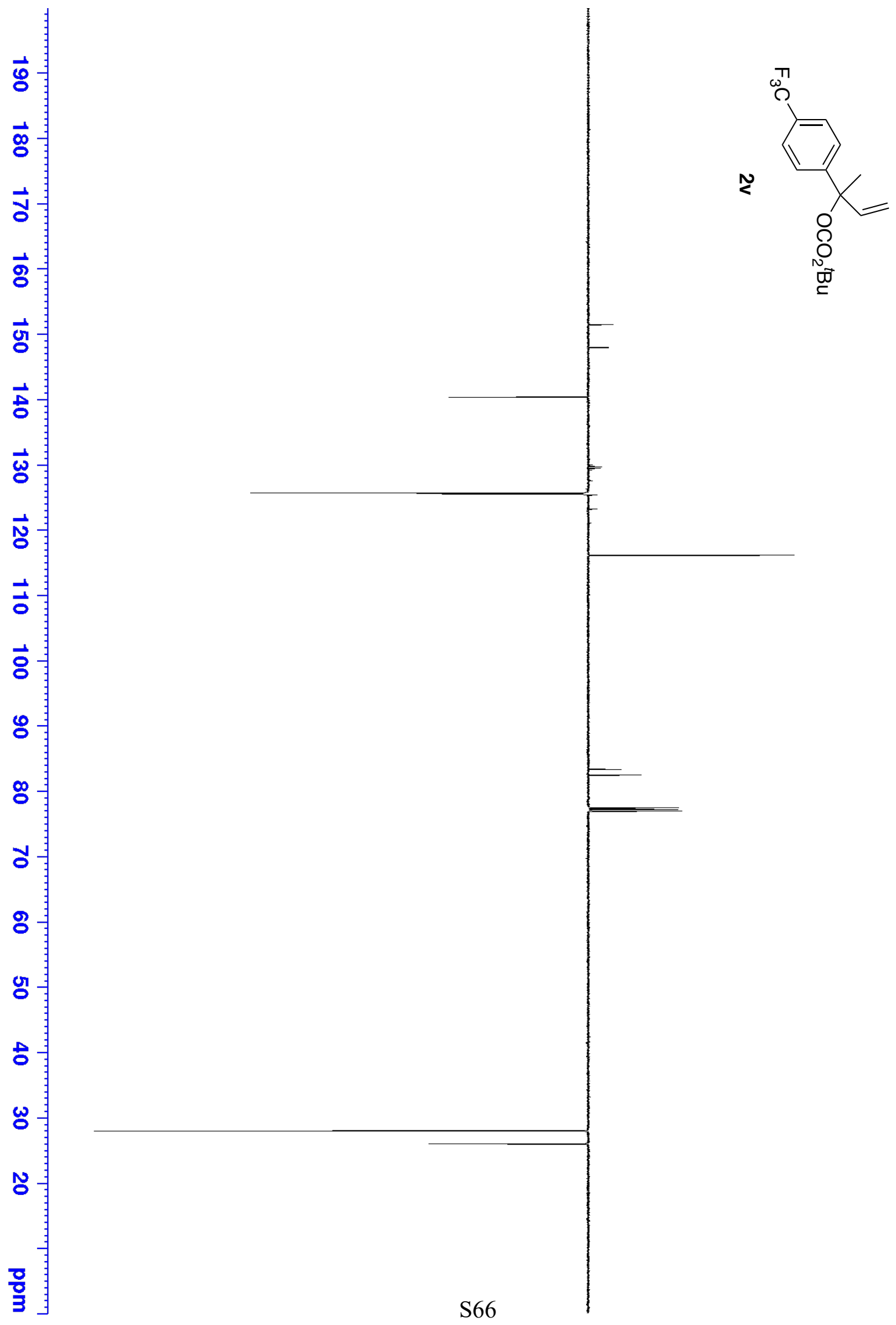




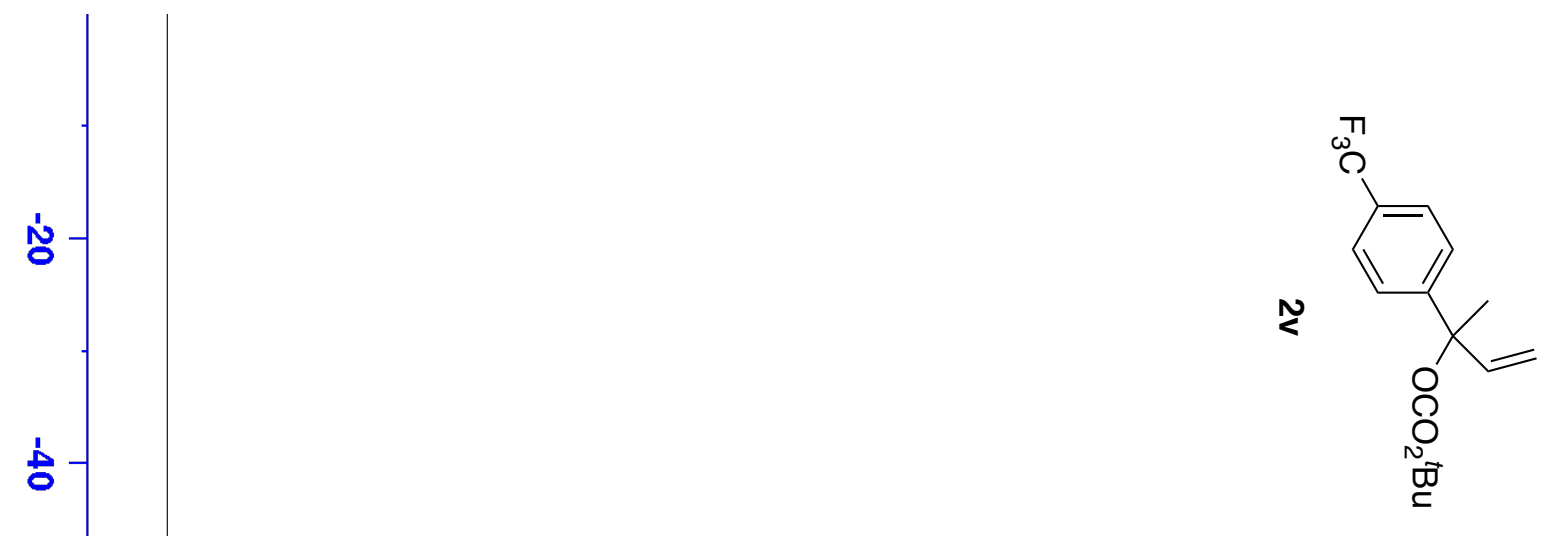

$\dot{\vec{s}}$

$\dot{\vec{v}}$

妾

के

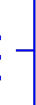

8

$\dot{\vec{g}}$

형

응 


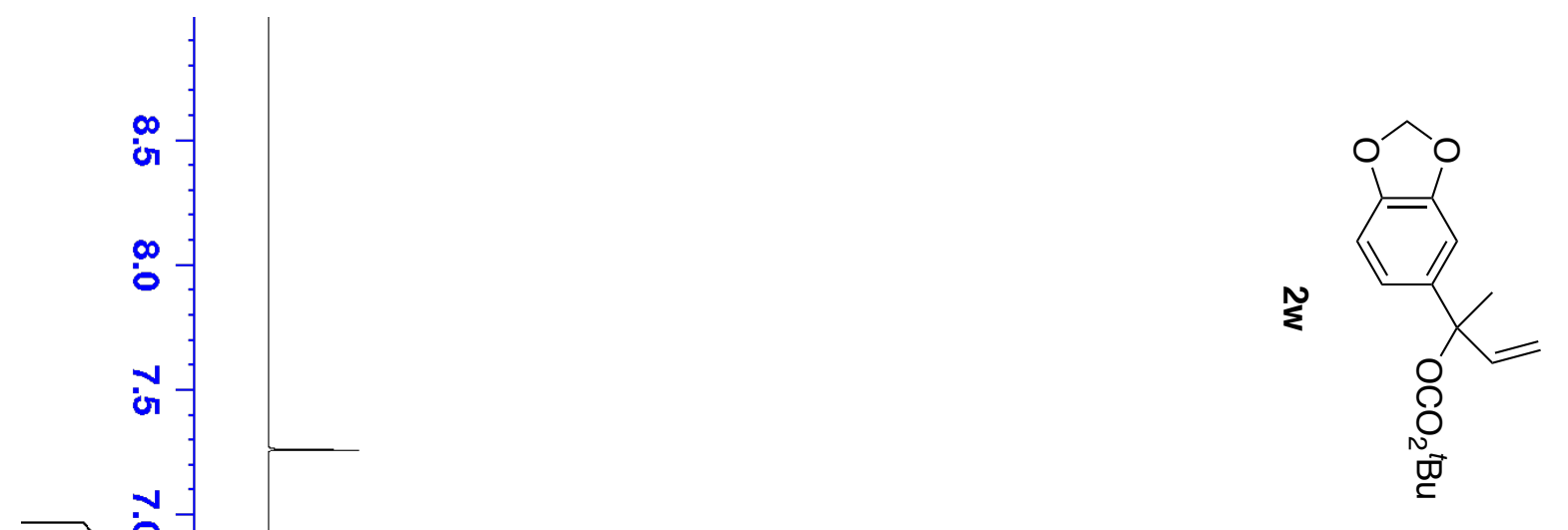



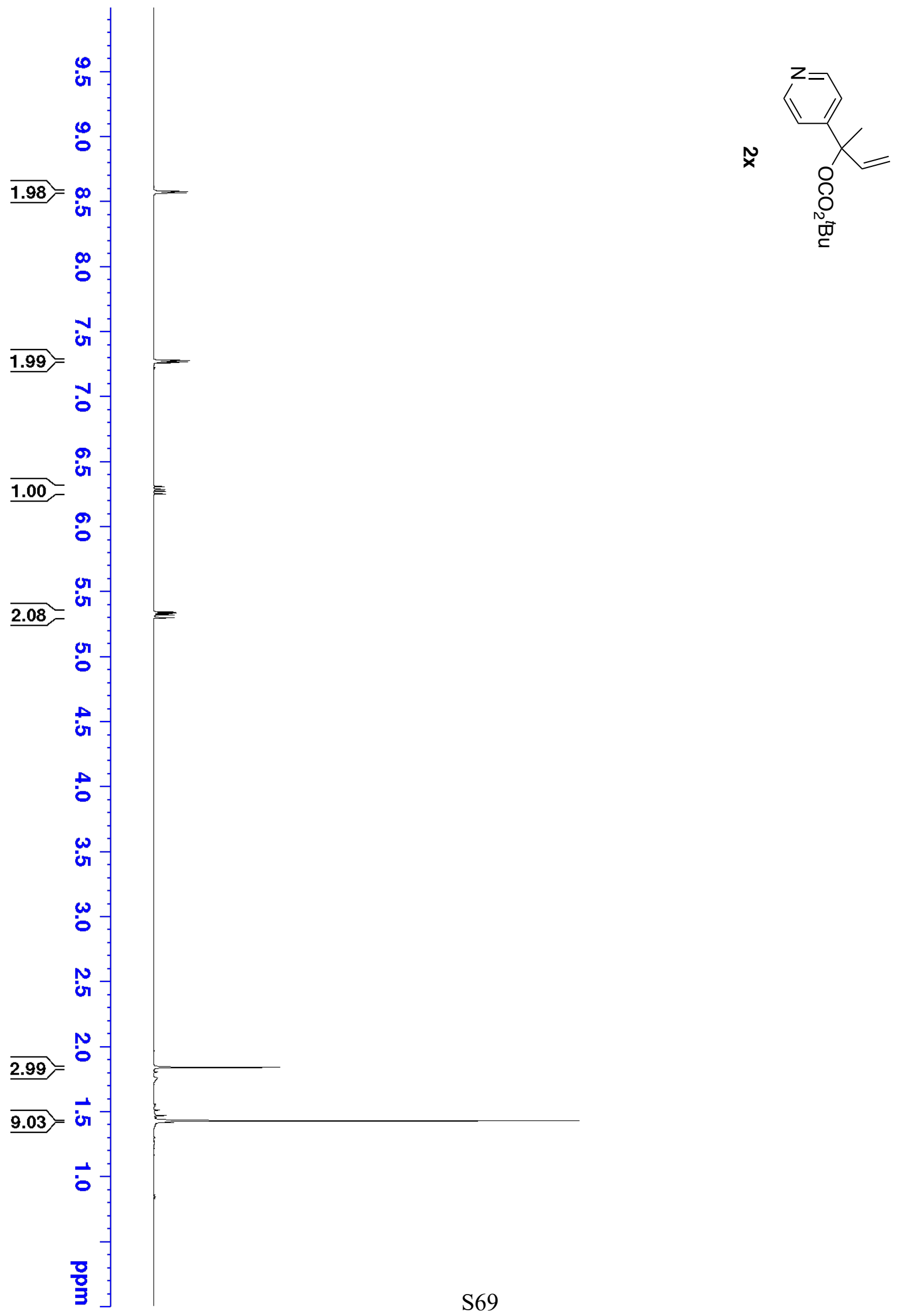


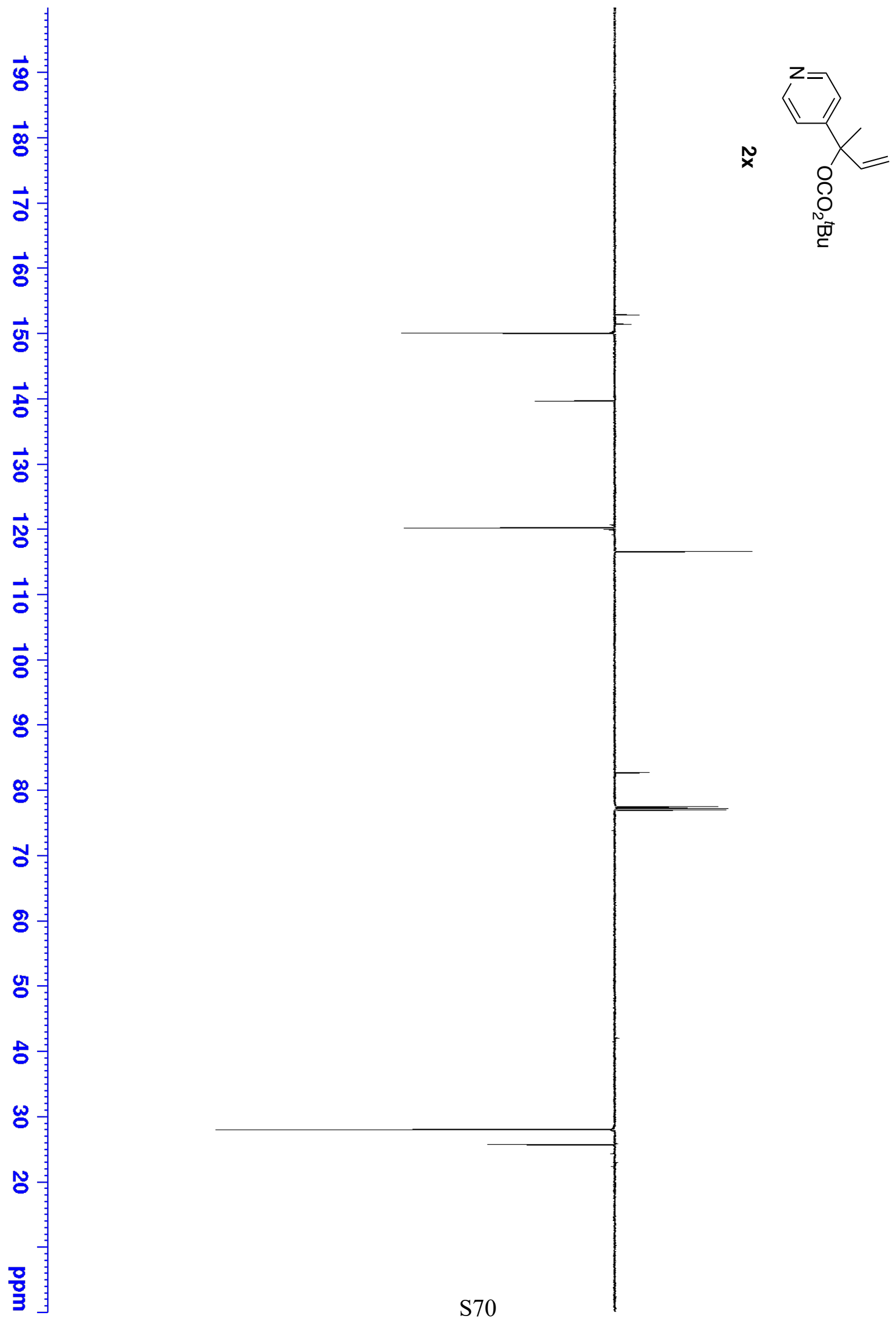




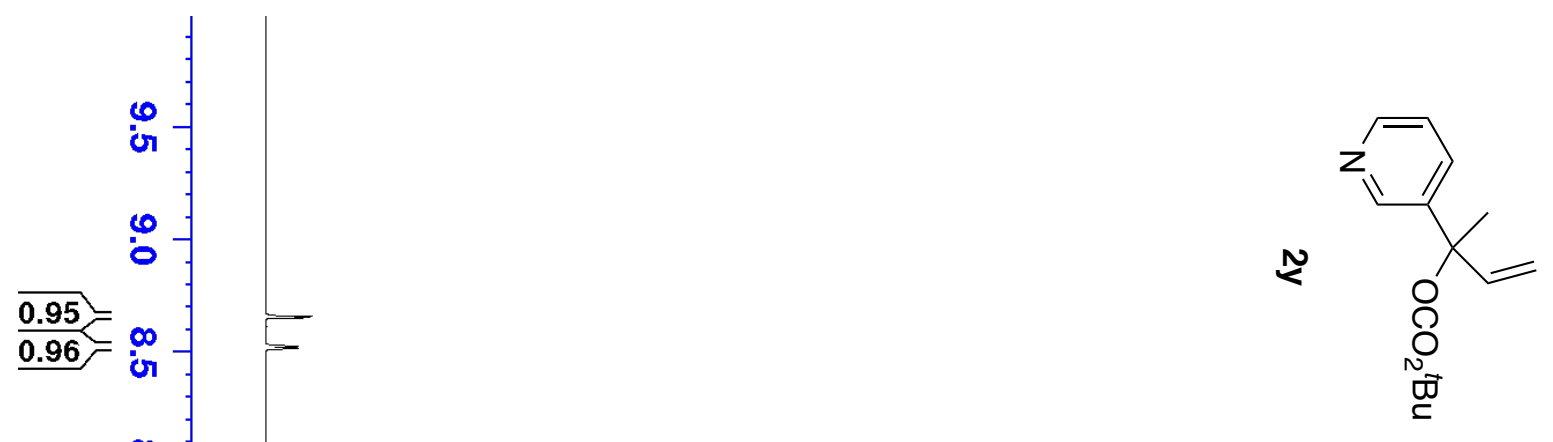




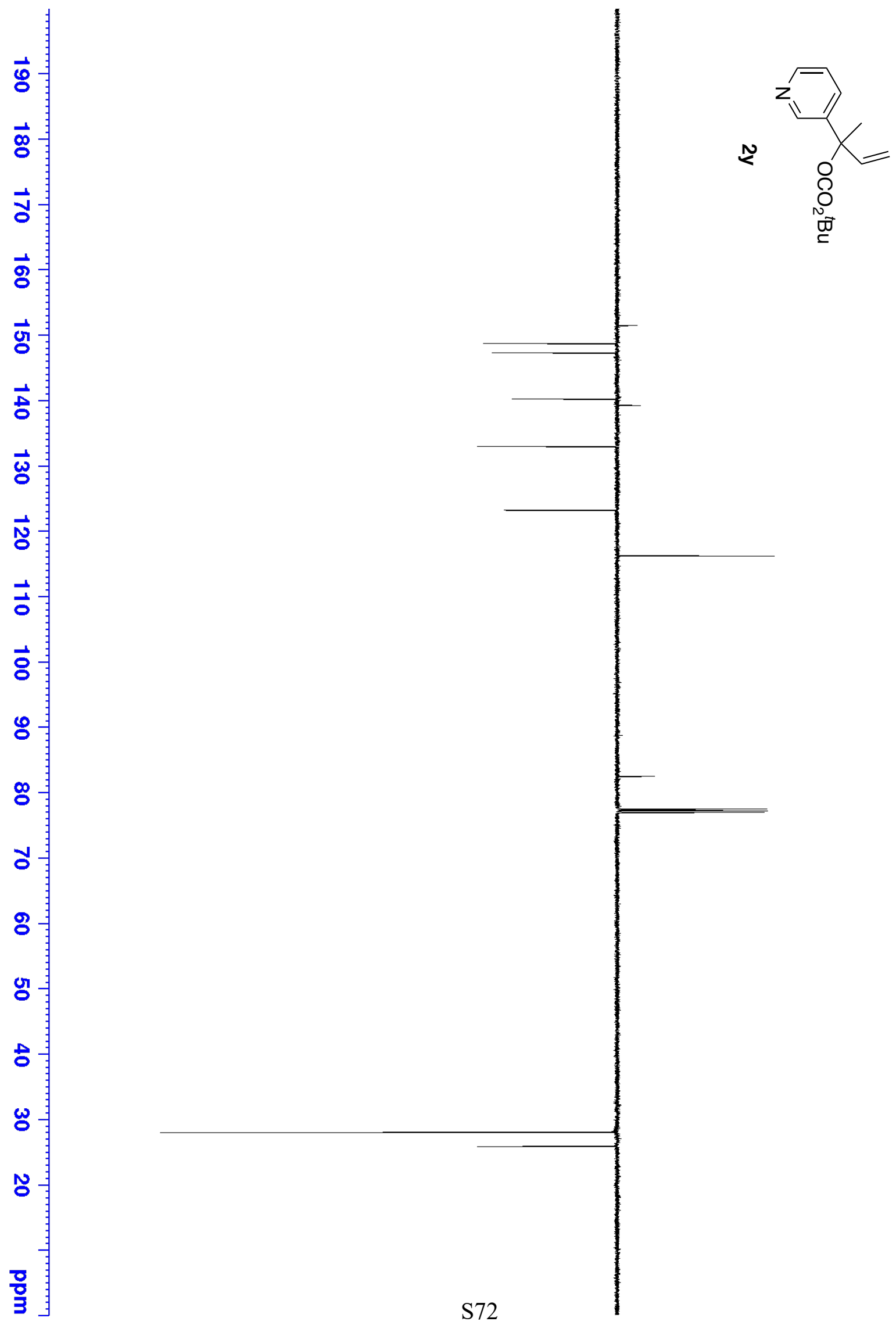




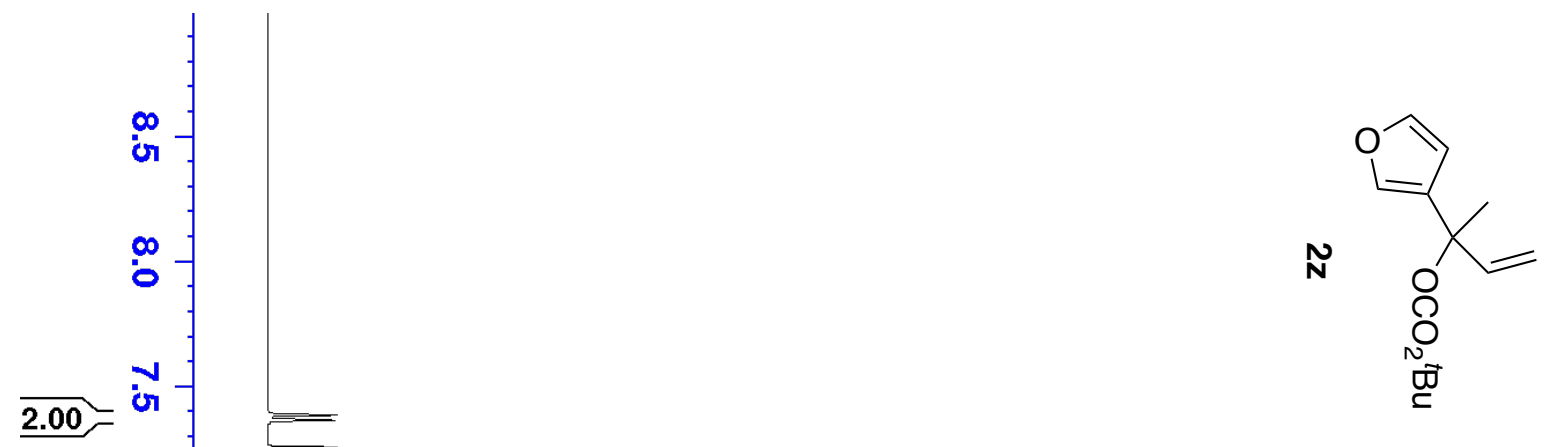




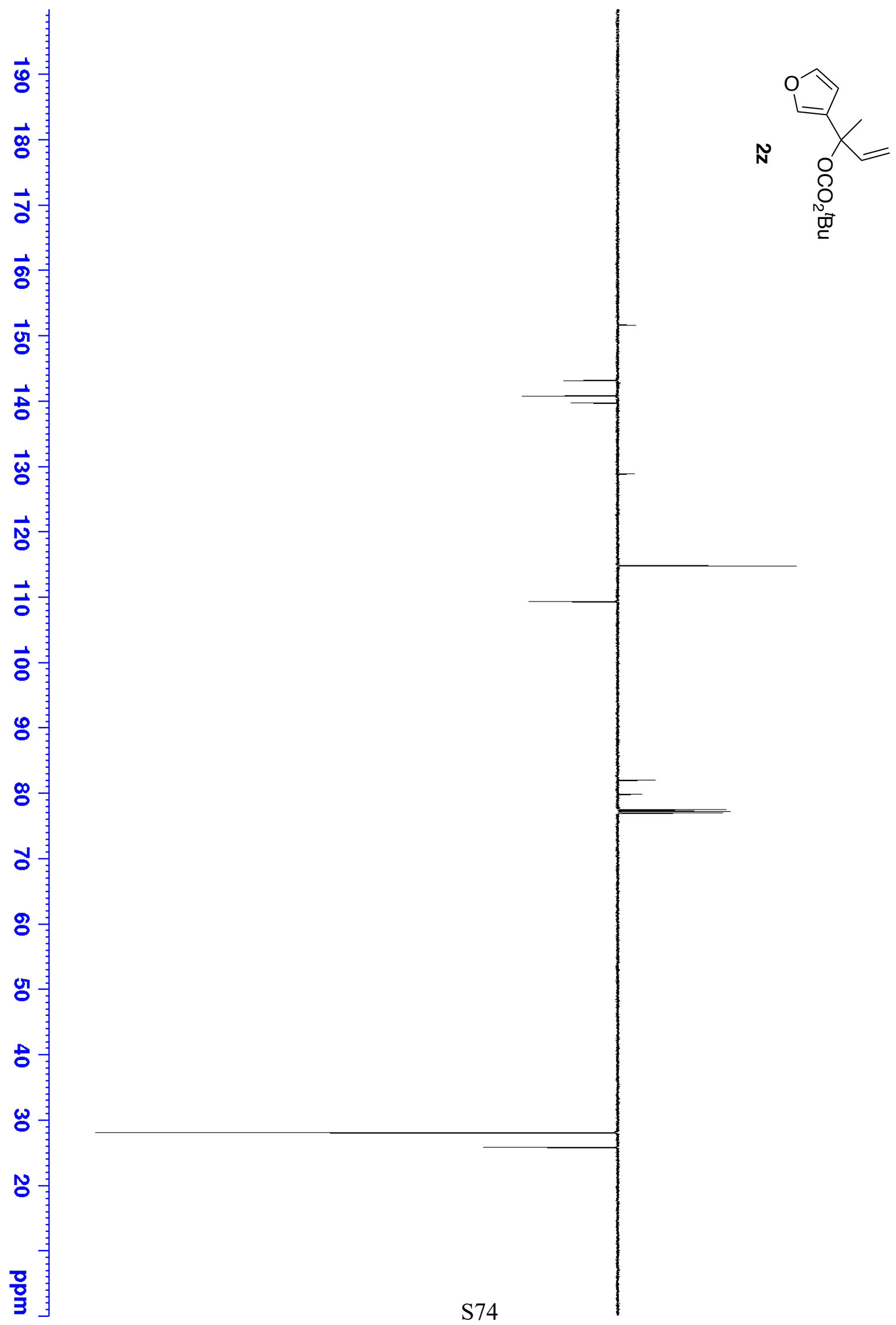




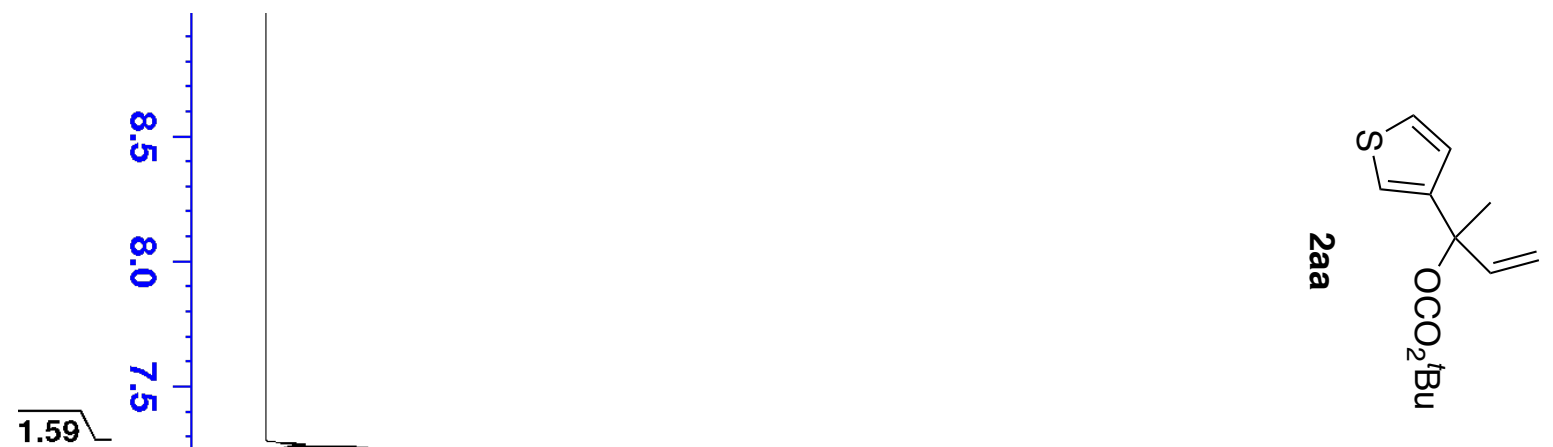




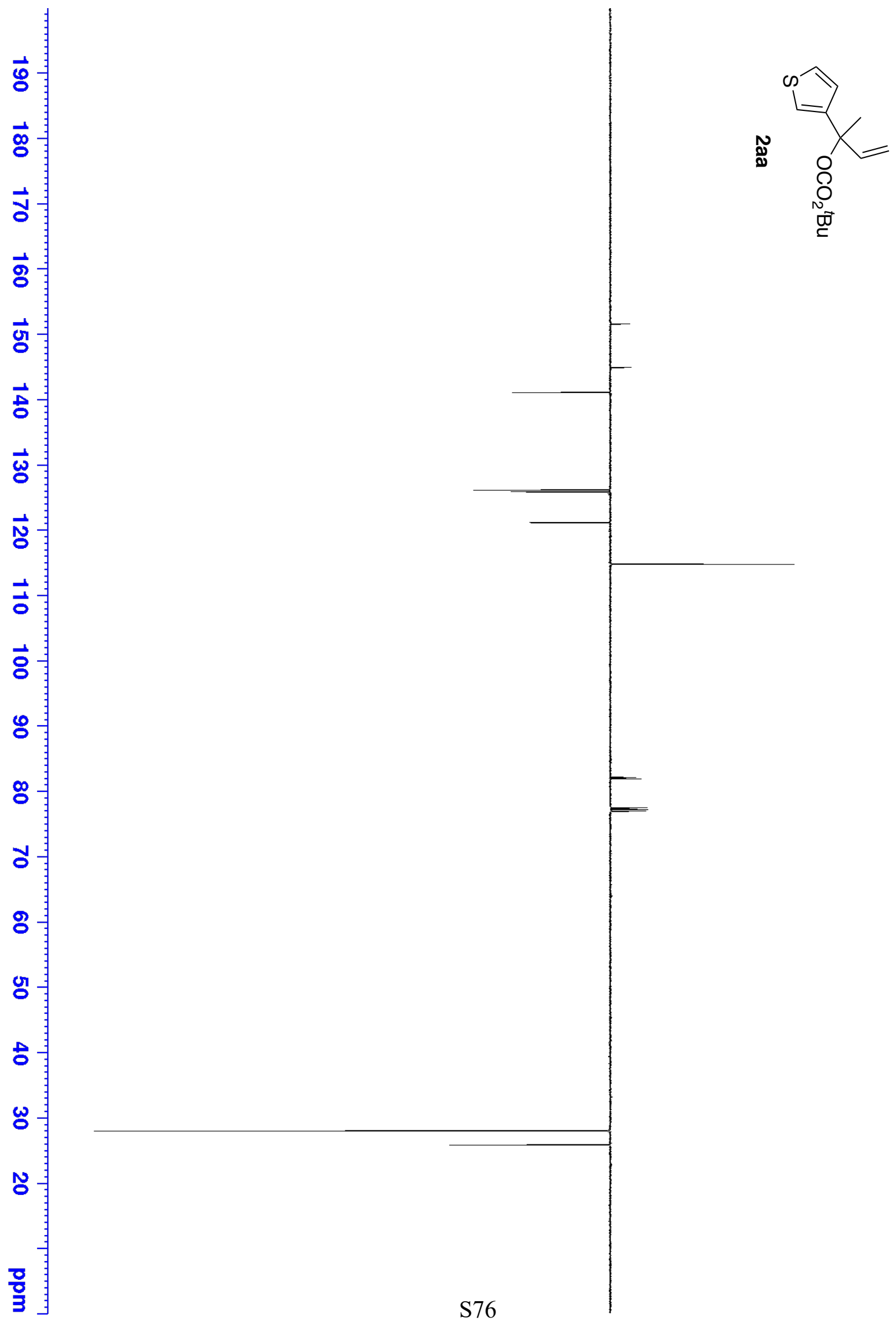




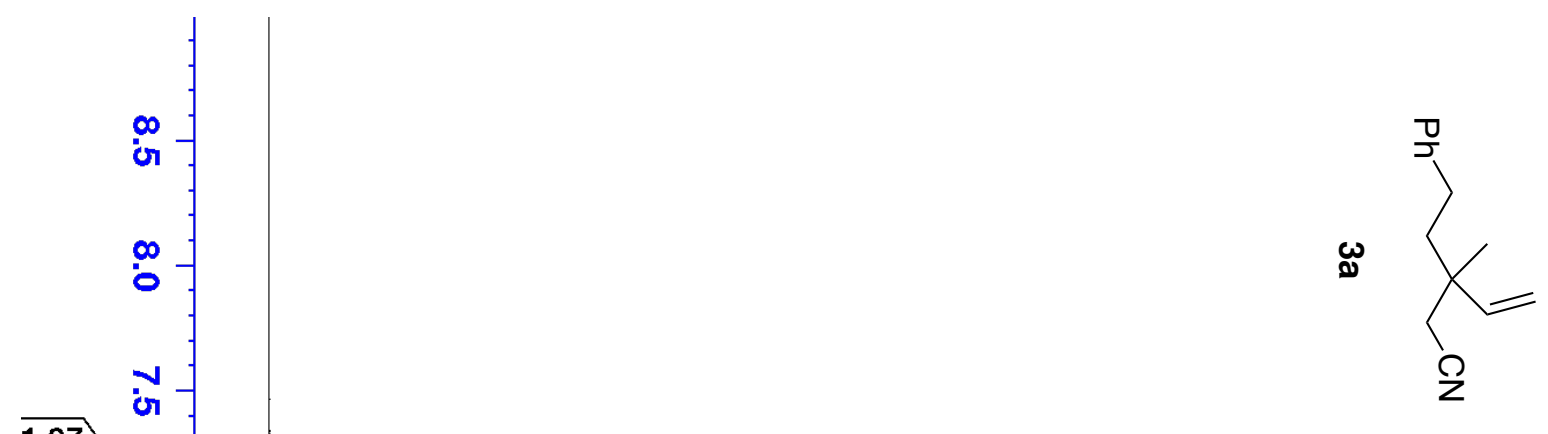$$
1.97
$$$$
2.98
$$$$
=
$$$$
\text { : }
$$

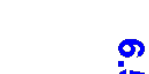

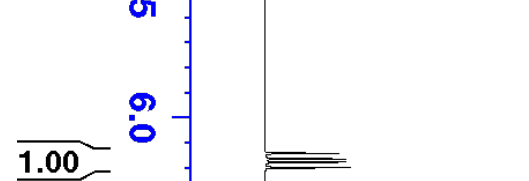




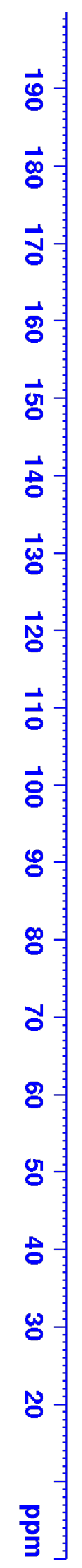
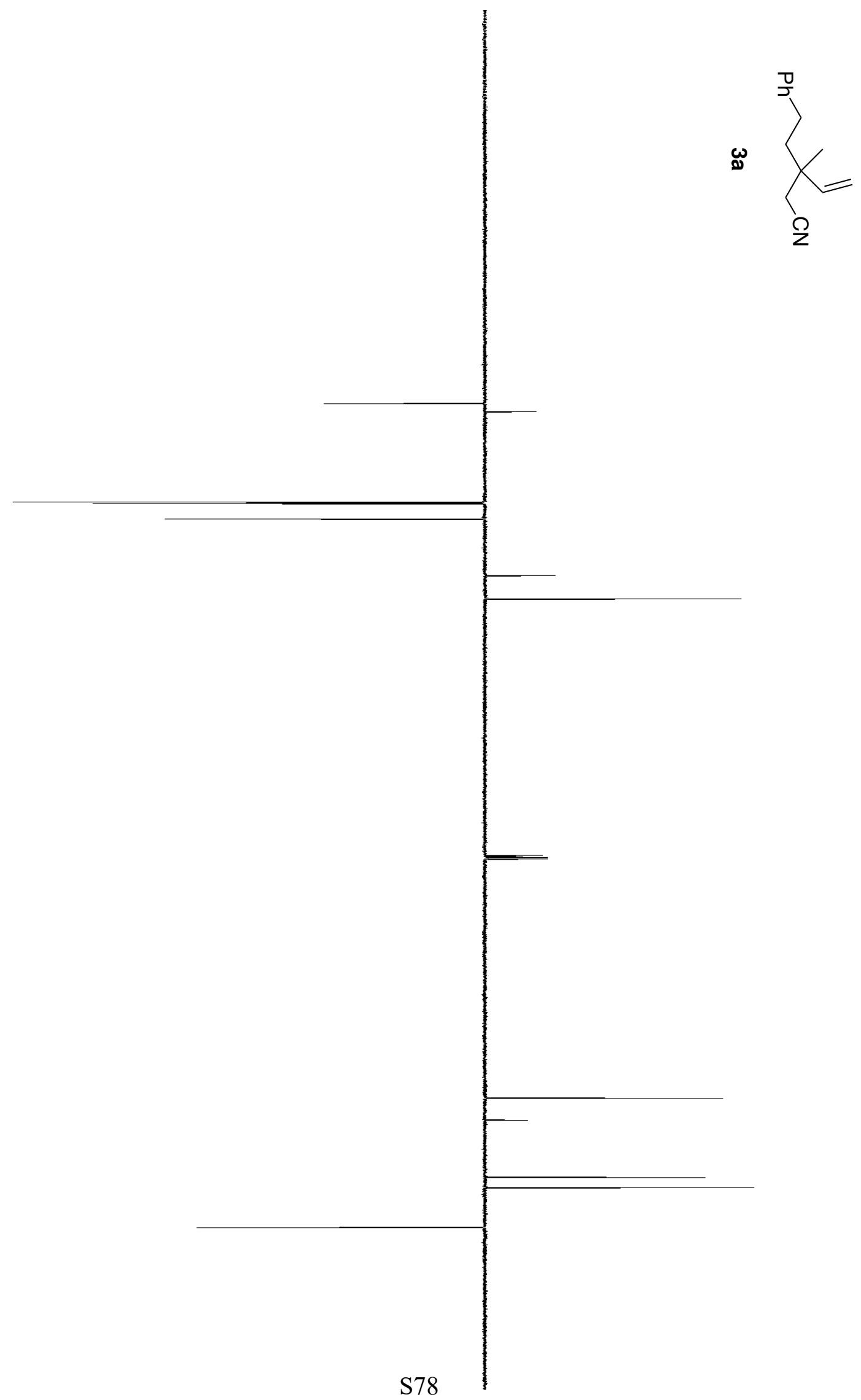


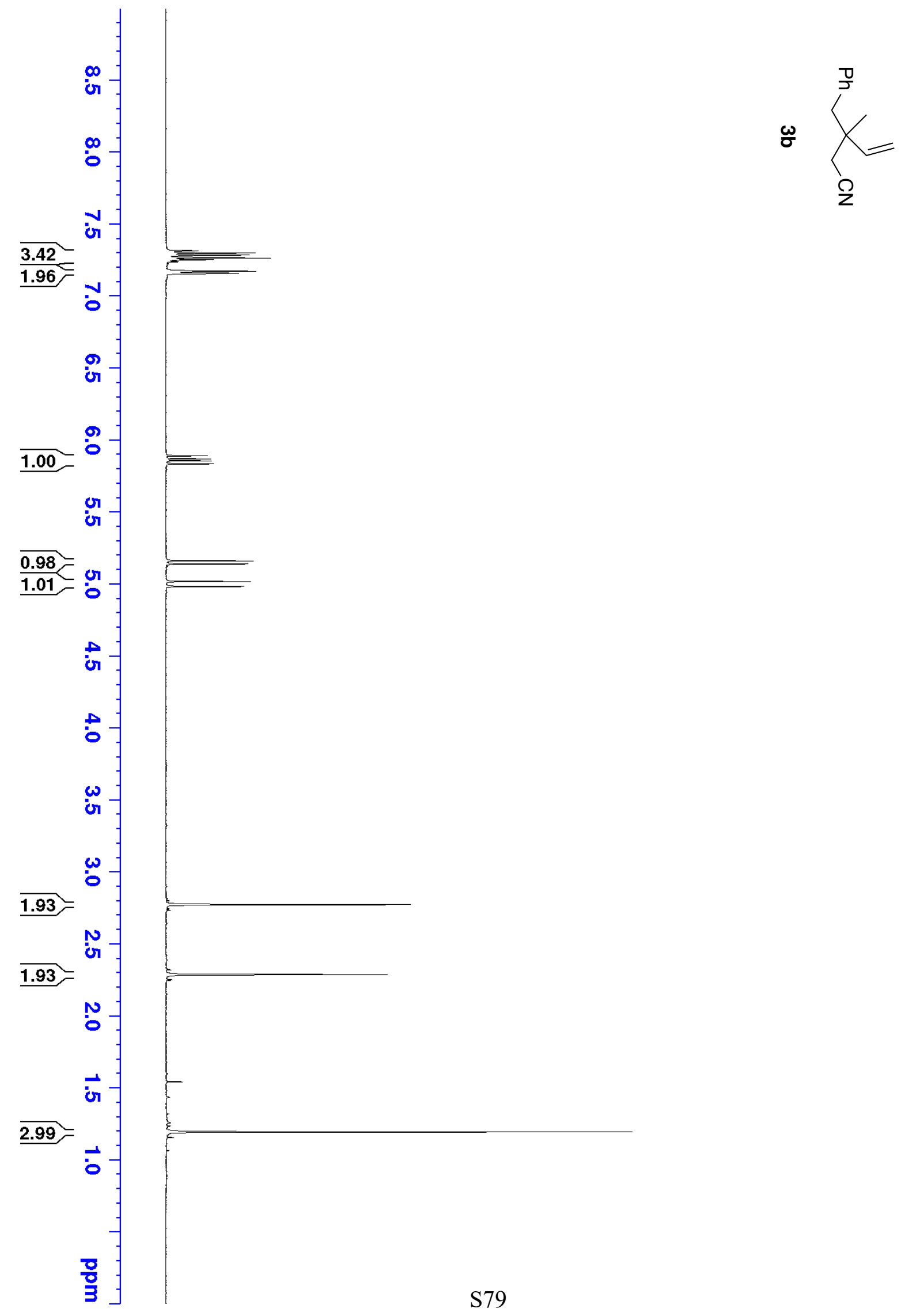




$$
H^{\prime \prime}
$$




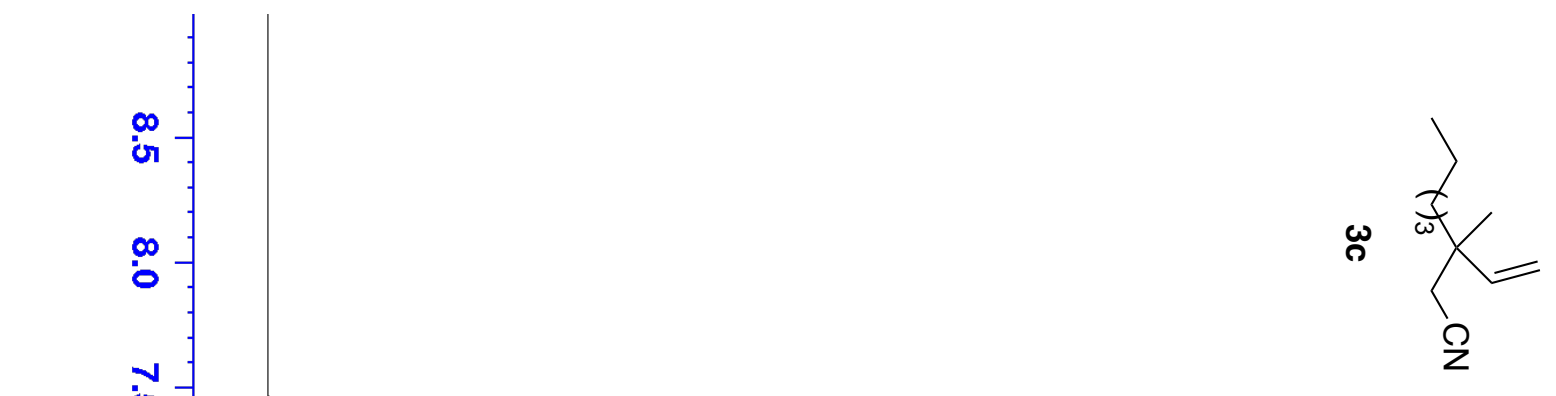




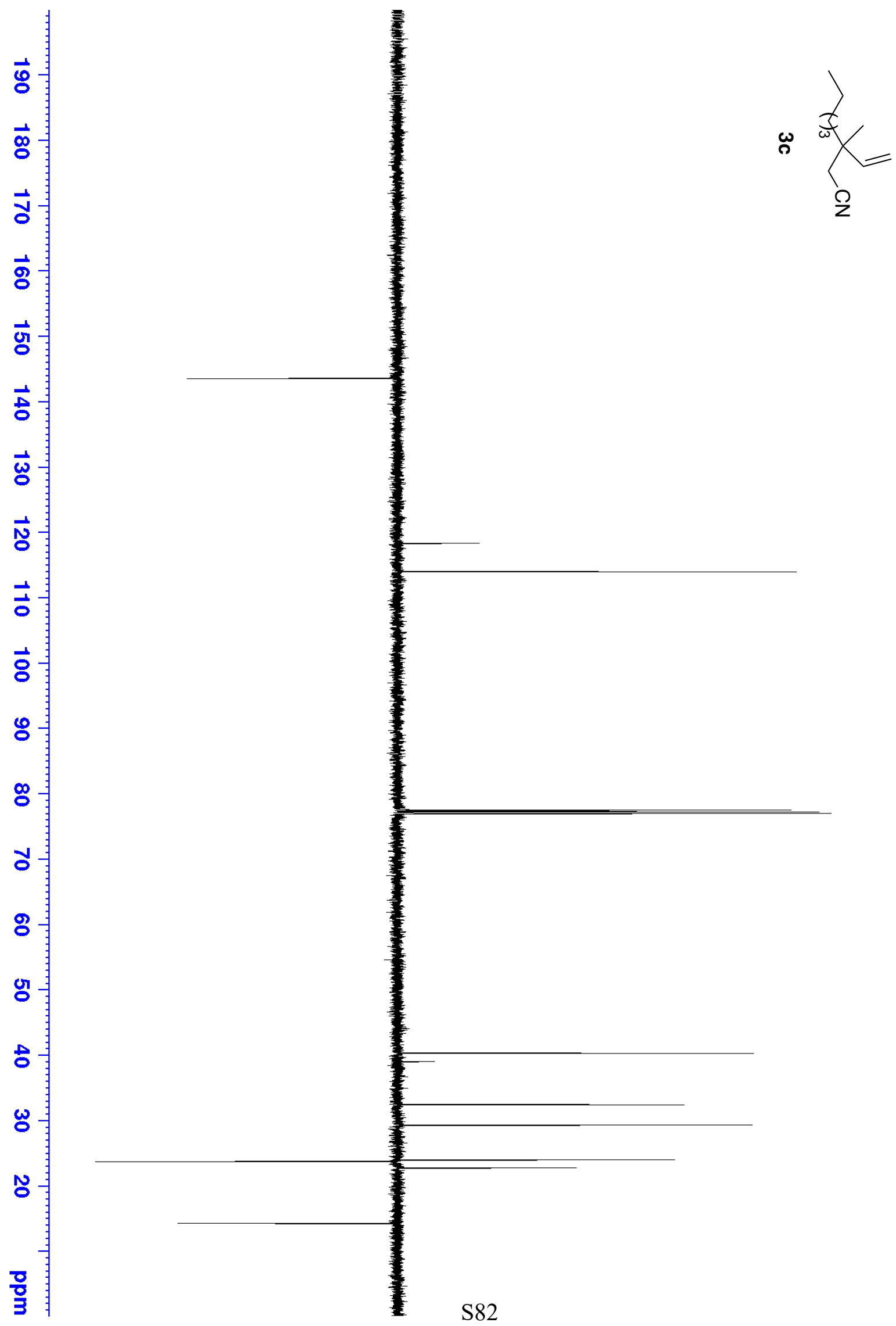




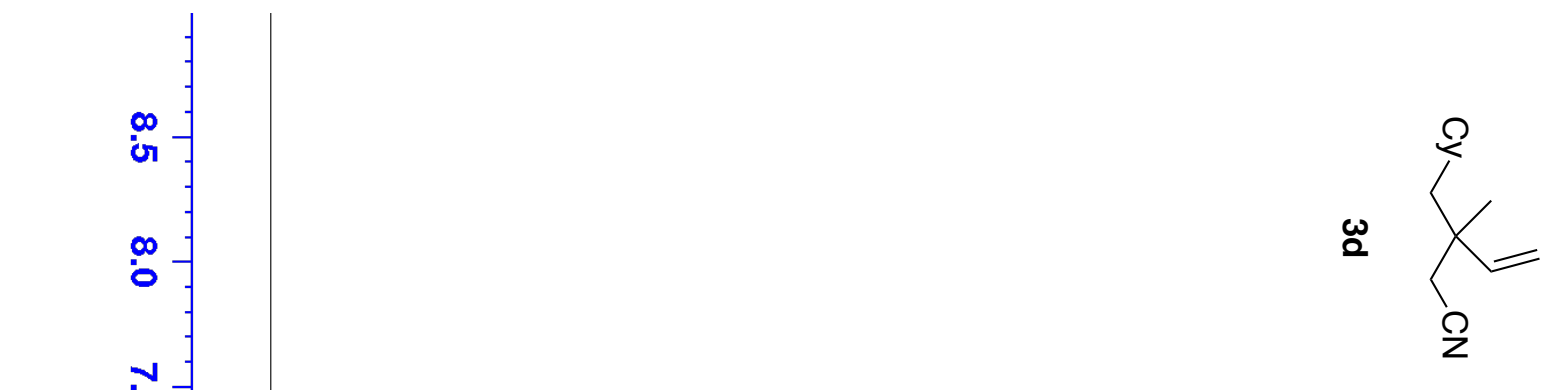

1.01

1.02 or

or

or

$\omega$

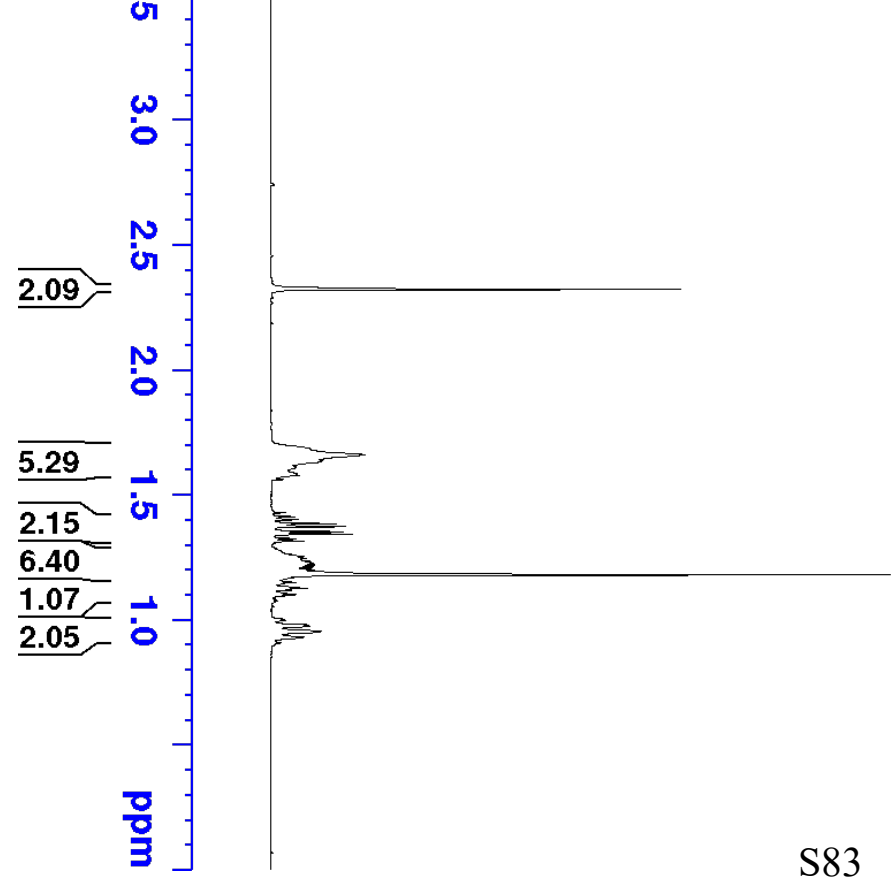




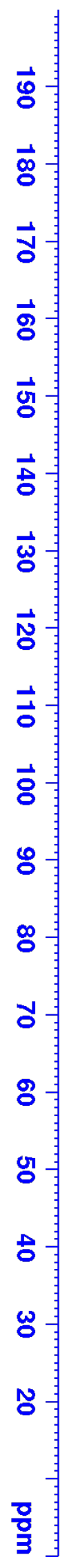
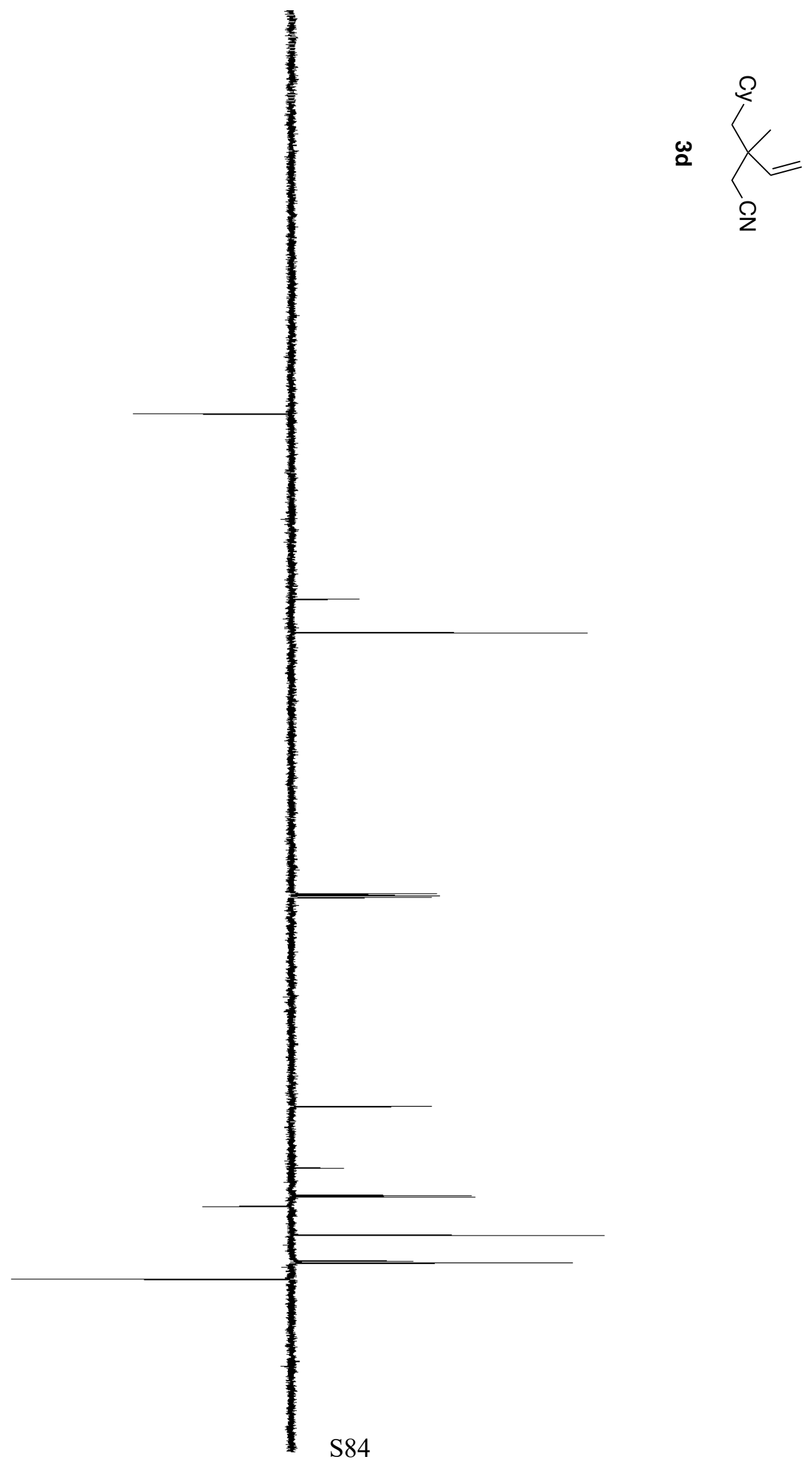


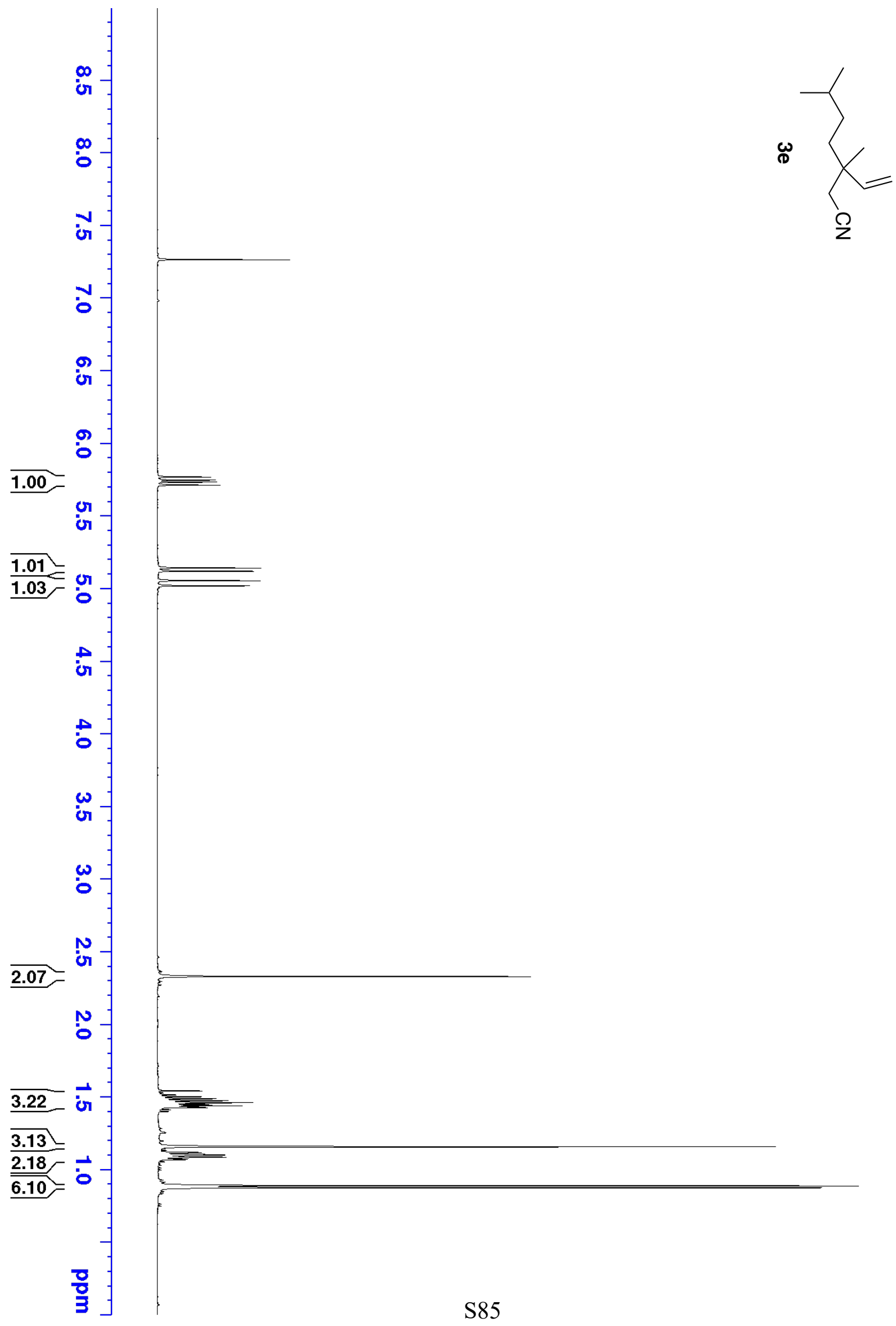




$$
\|{ }^{\prime}
$$




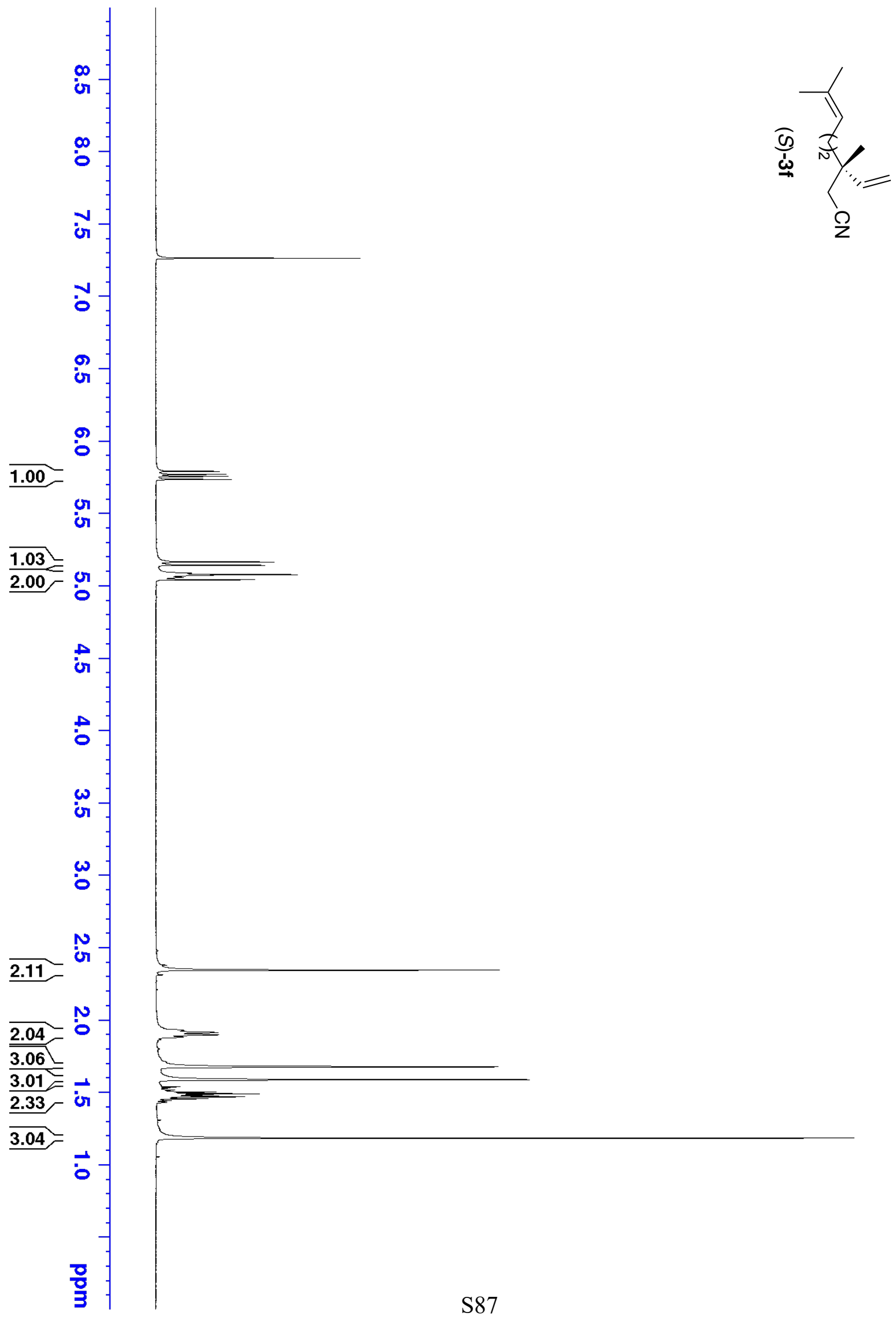




$$
\|
$$




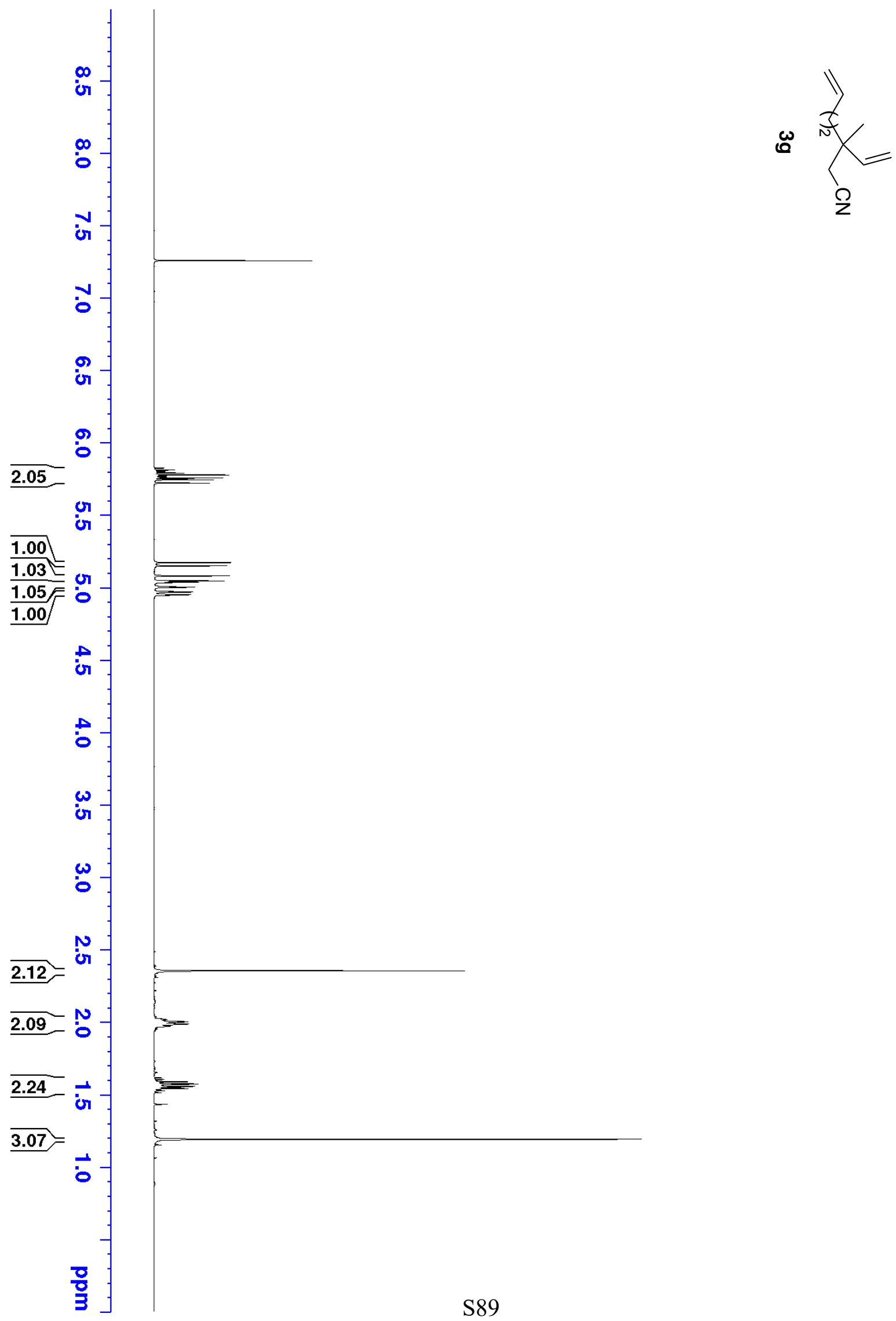




$$
\|
$$




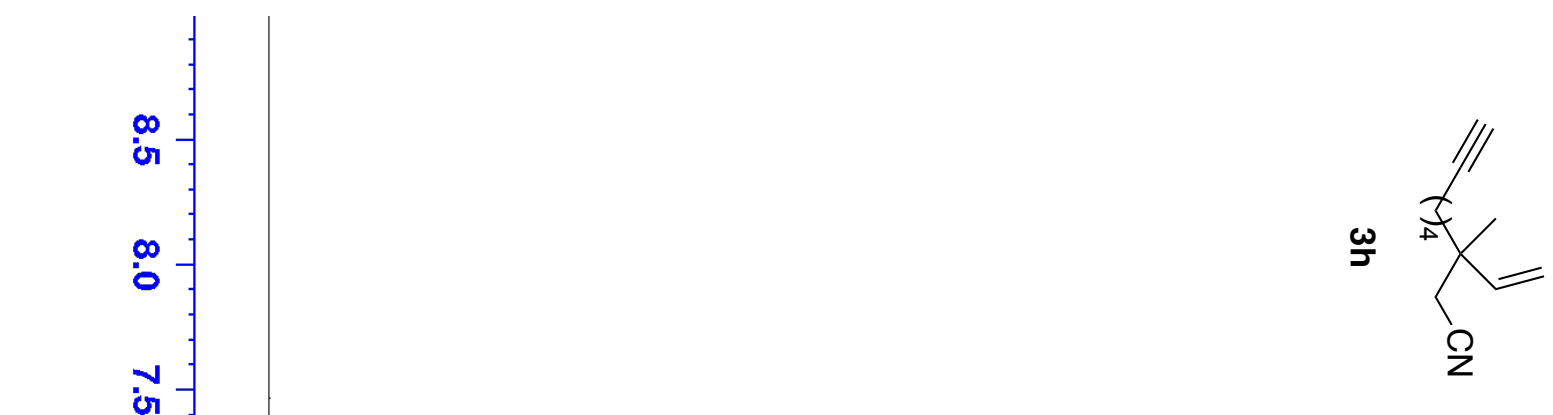




$$
\|=
$$



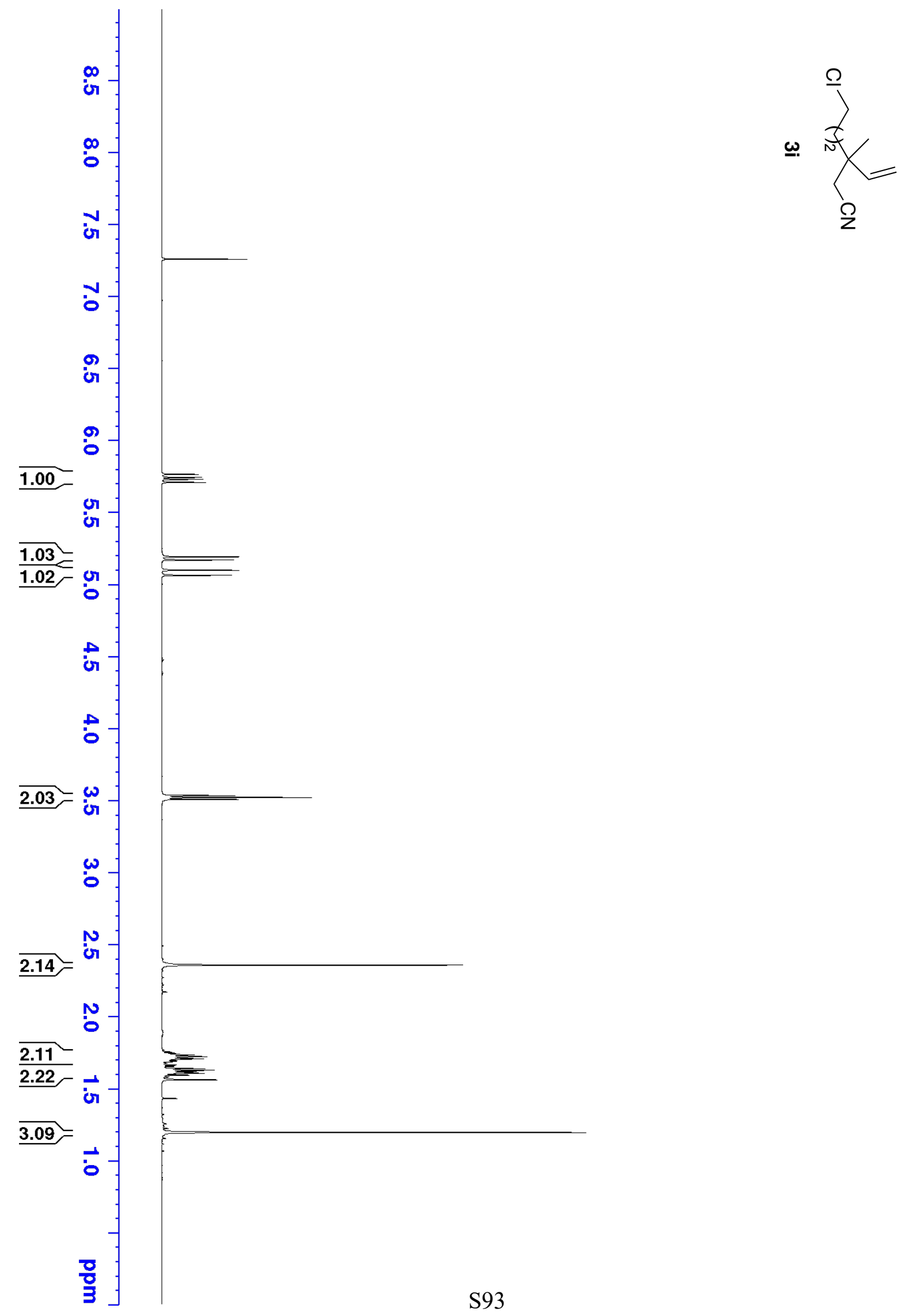


$$
\|=
$$




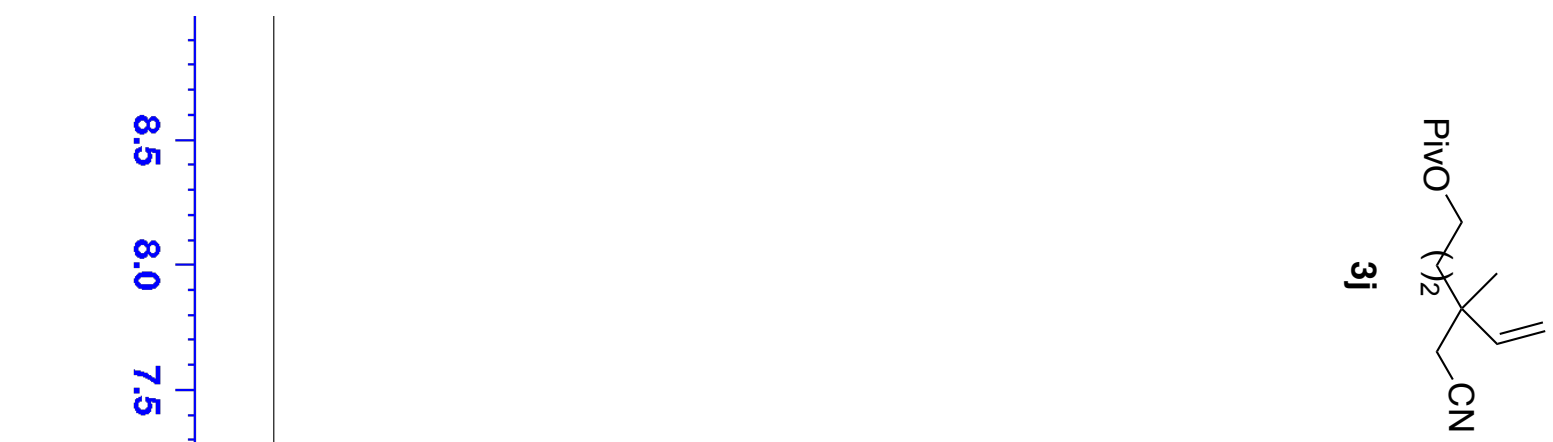




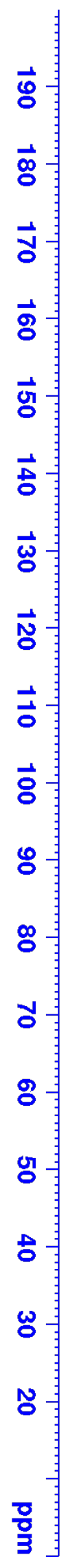

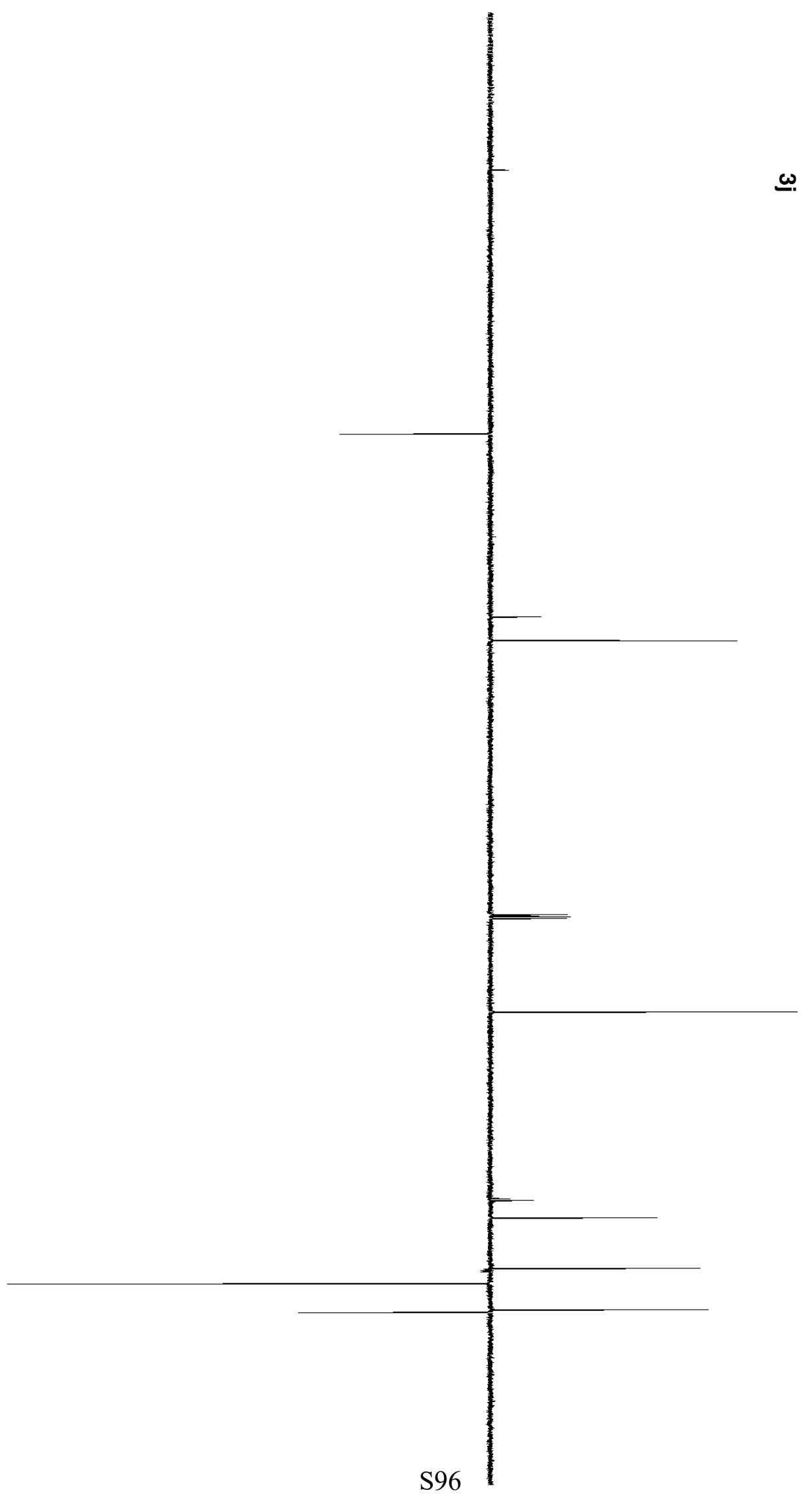




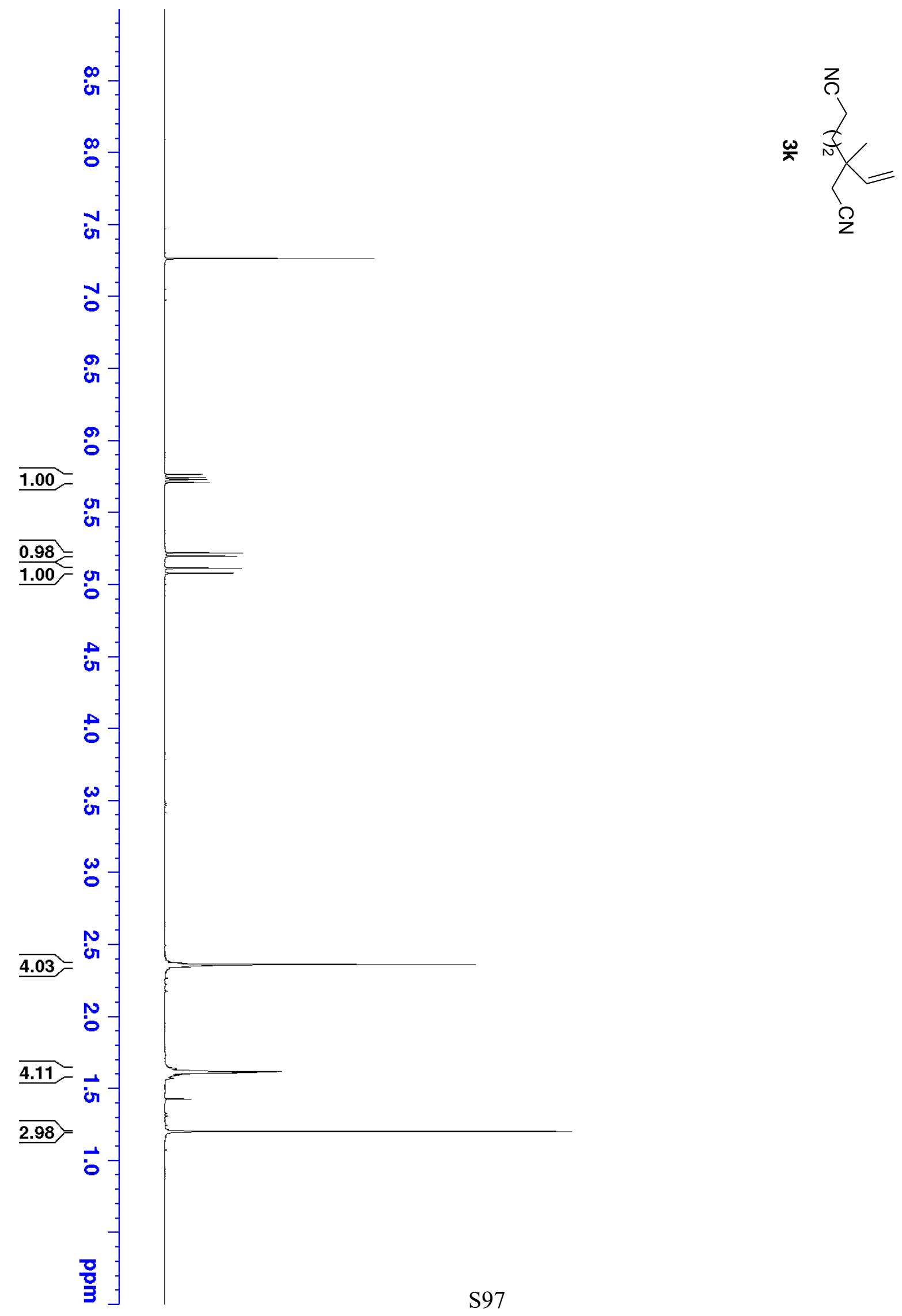




$$
1
$$




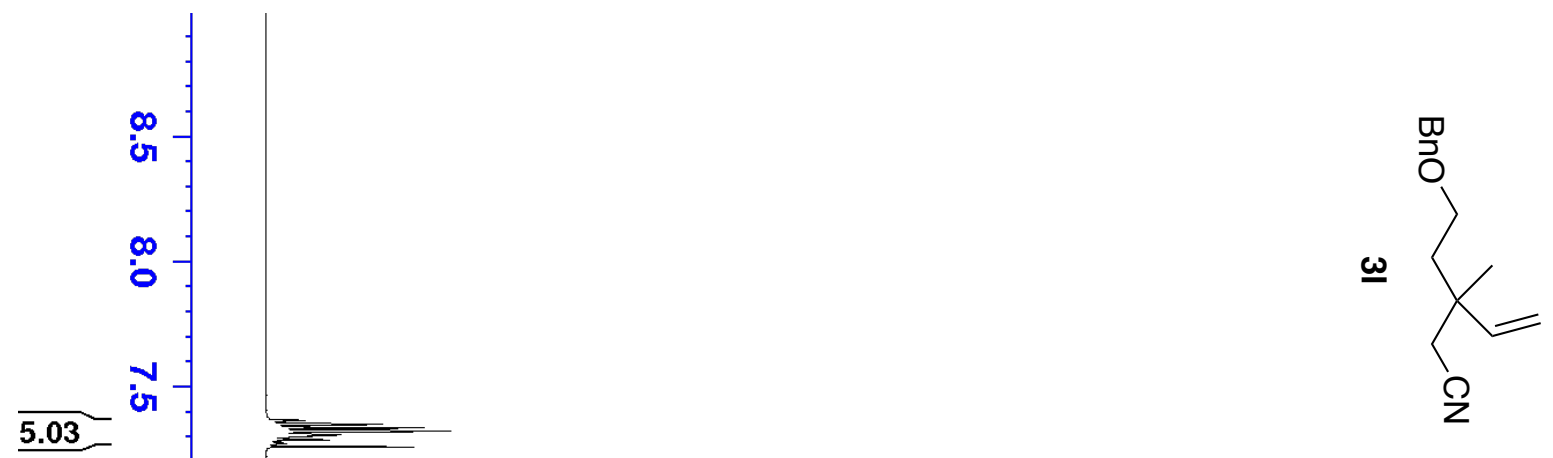




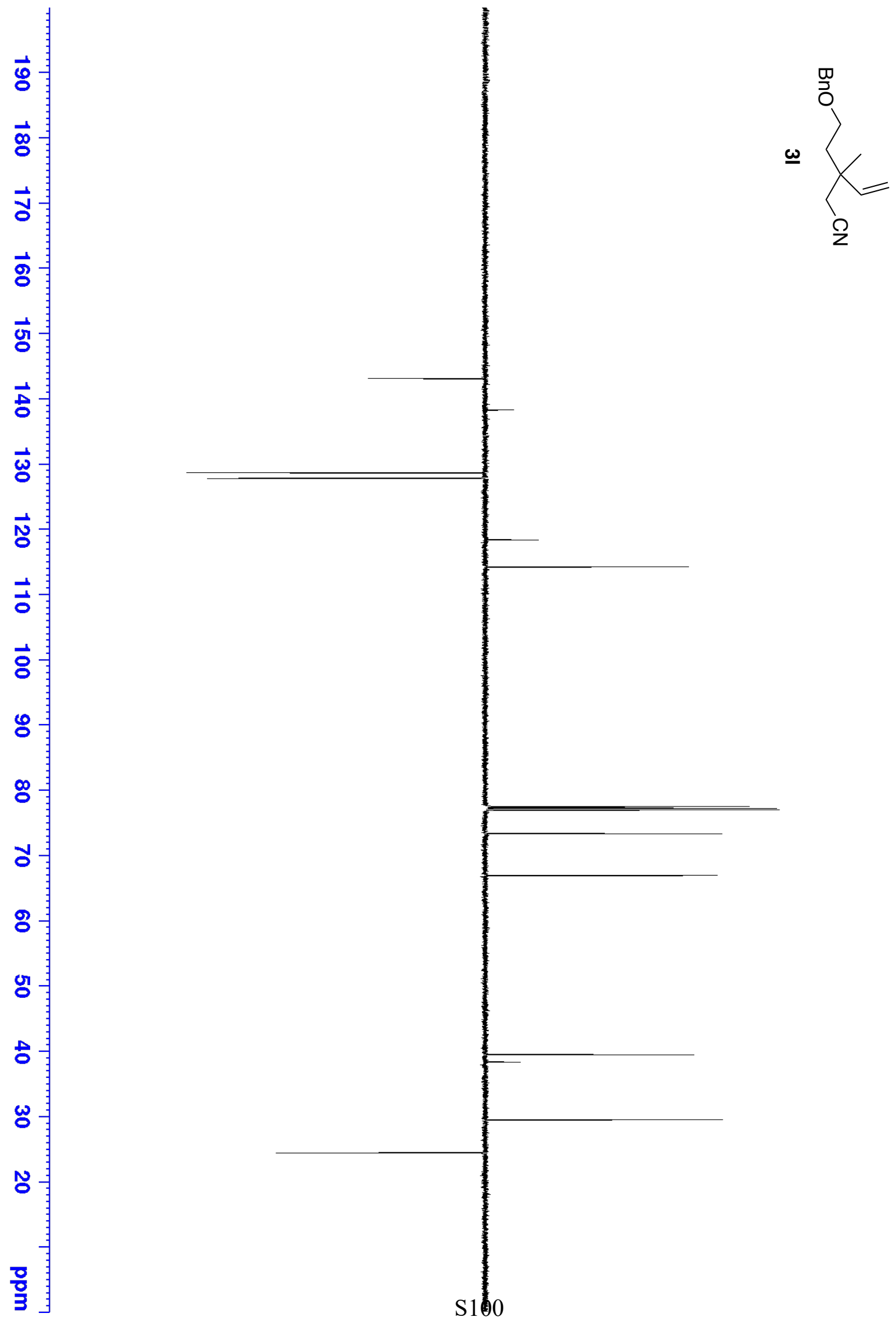



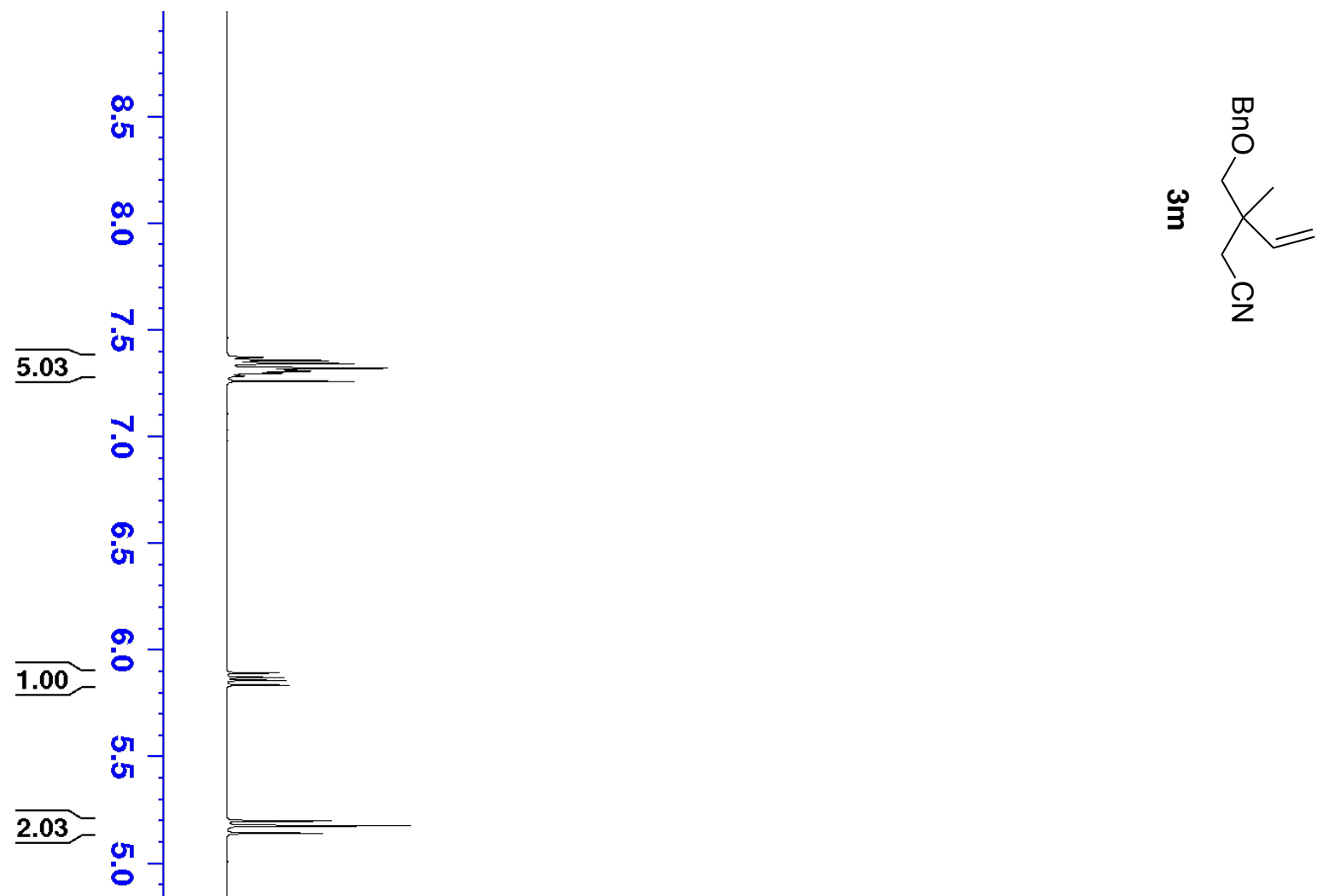

$\underline{2.01}=\stackrel{\circ}{\mathrm{or}}$
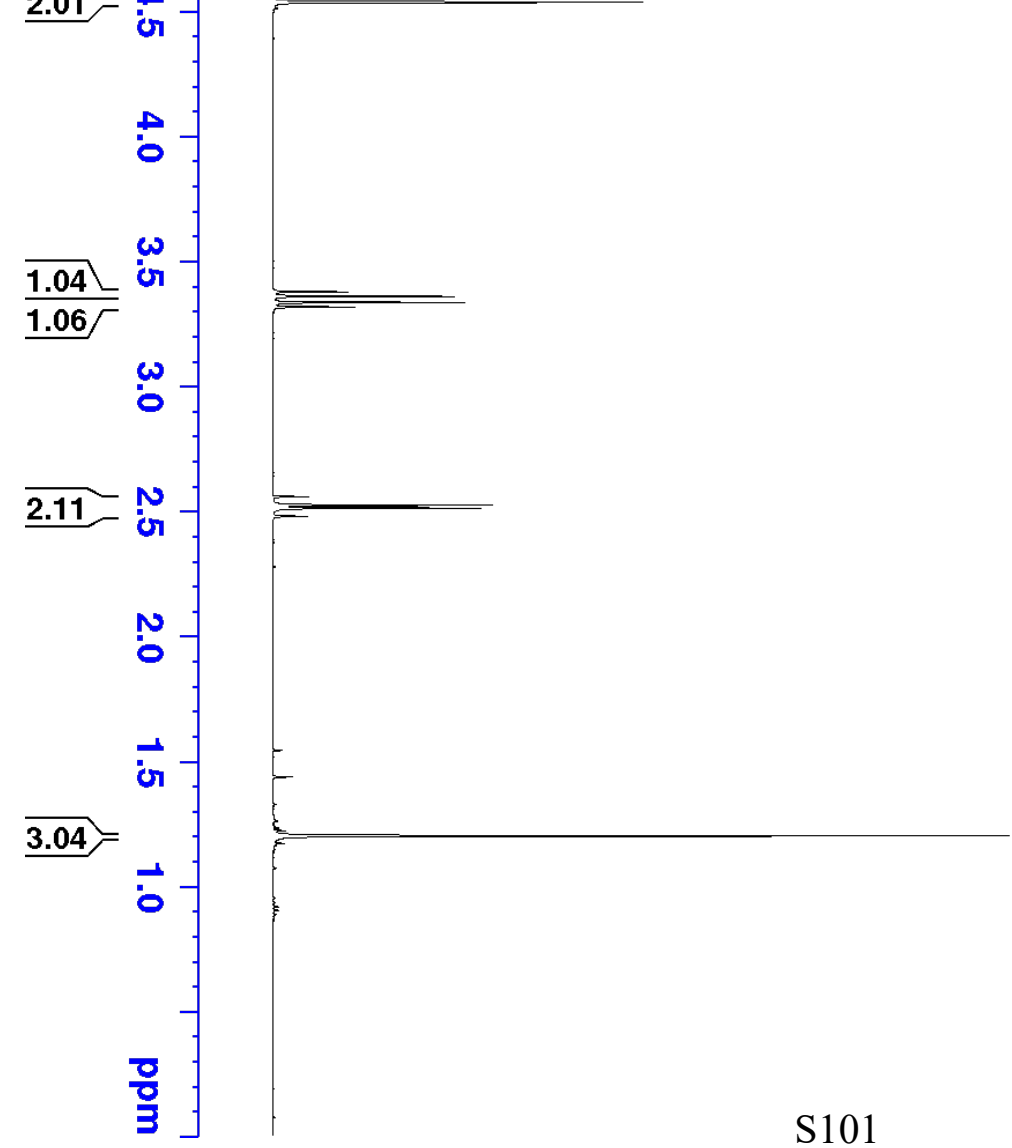


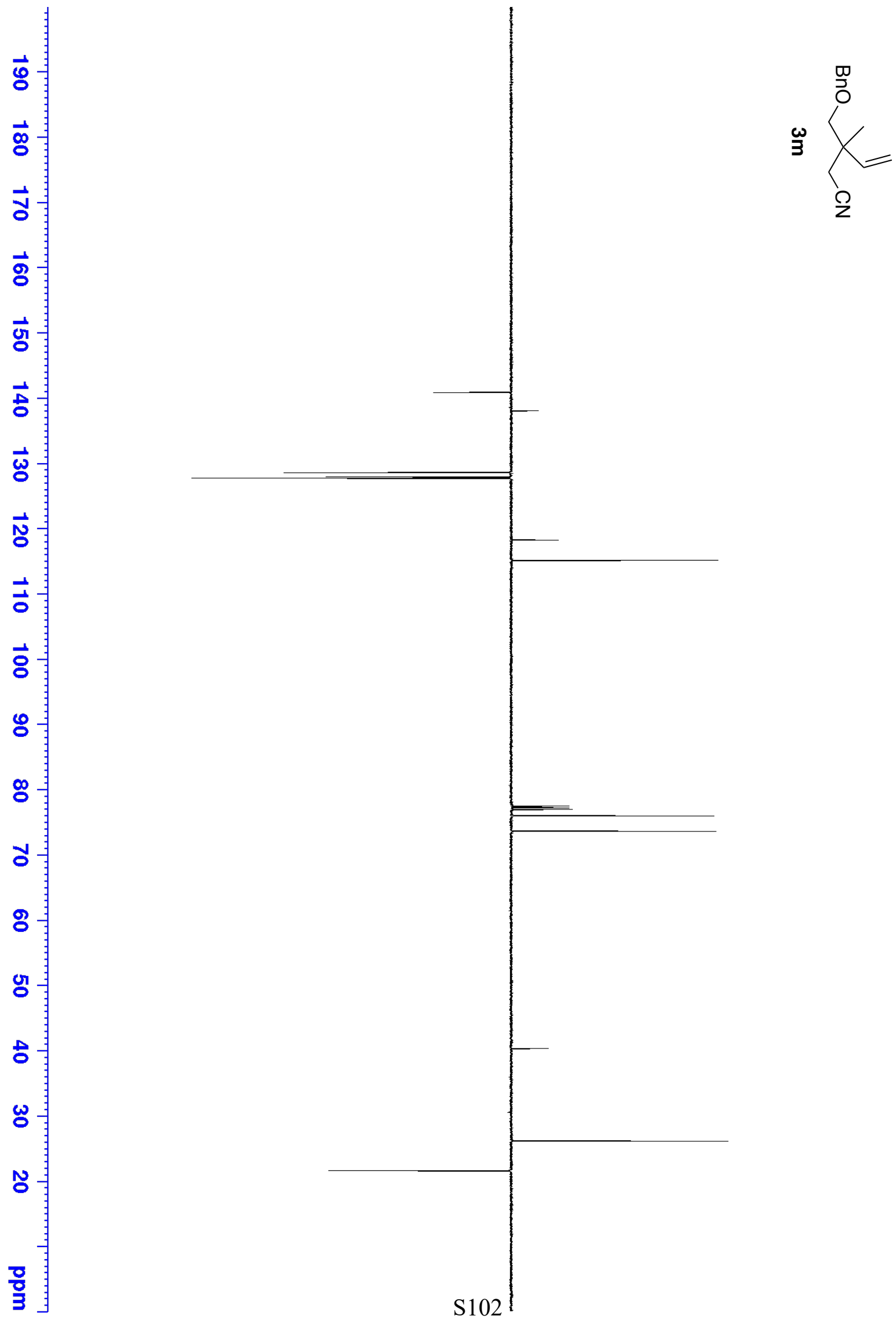




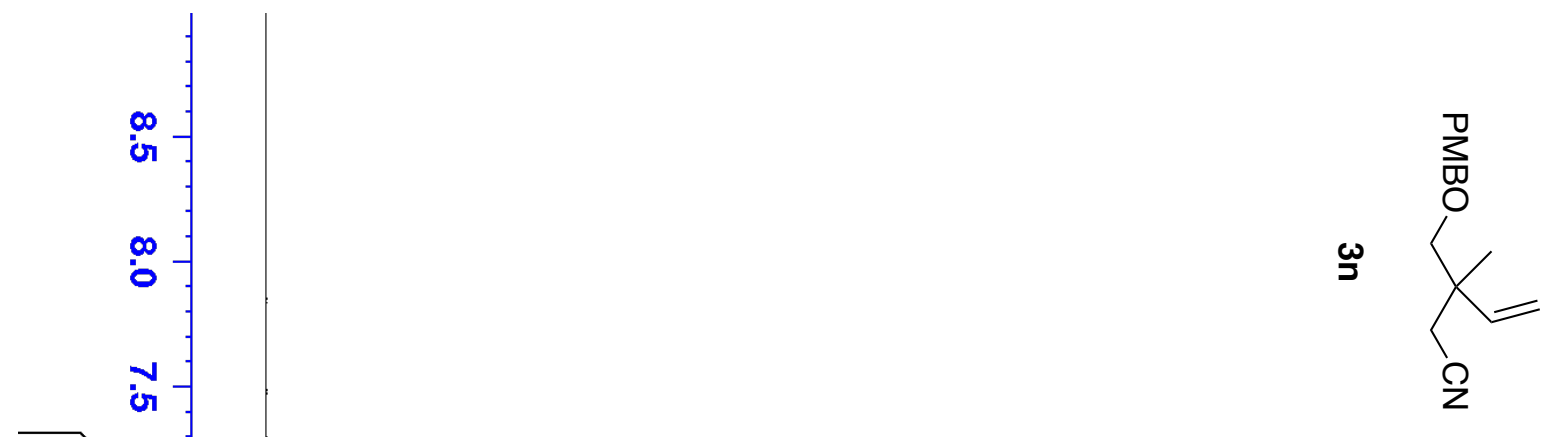




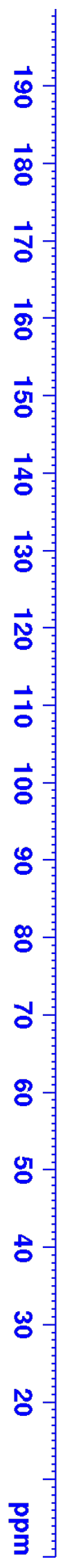
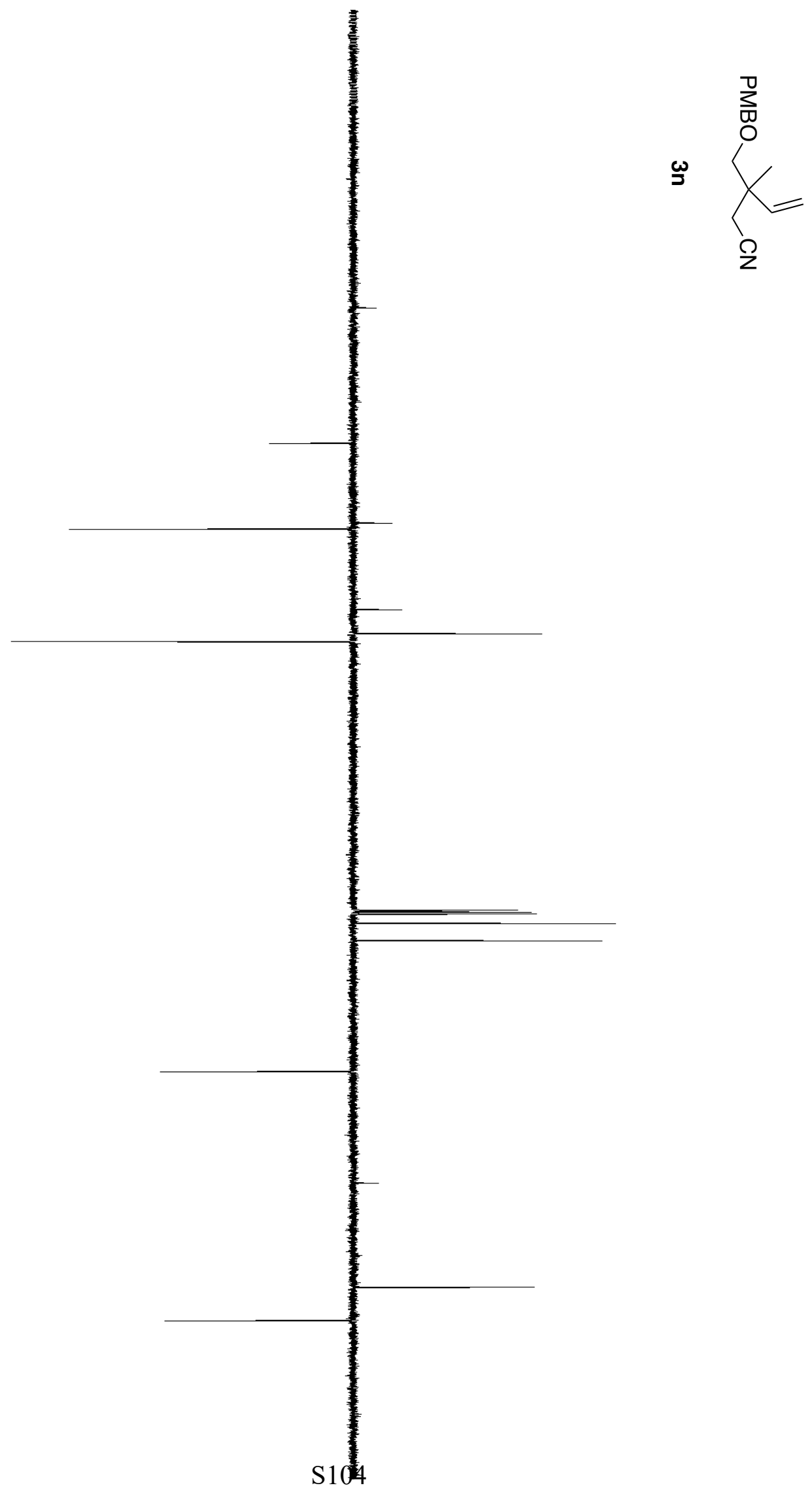


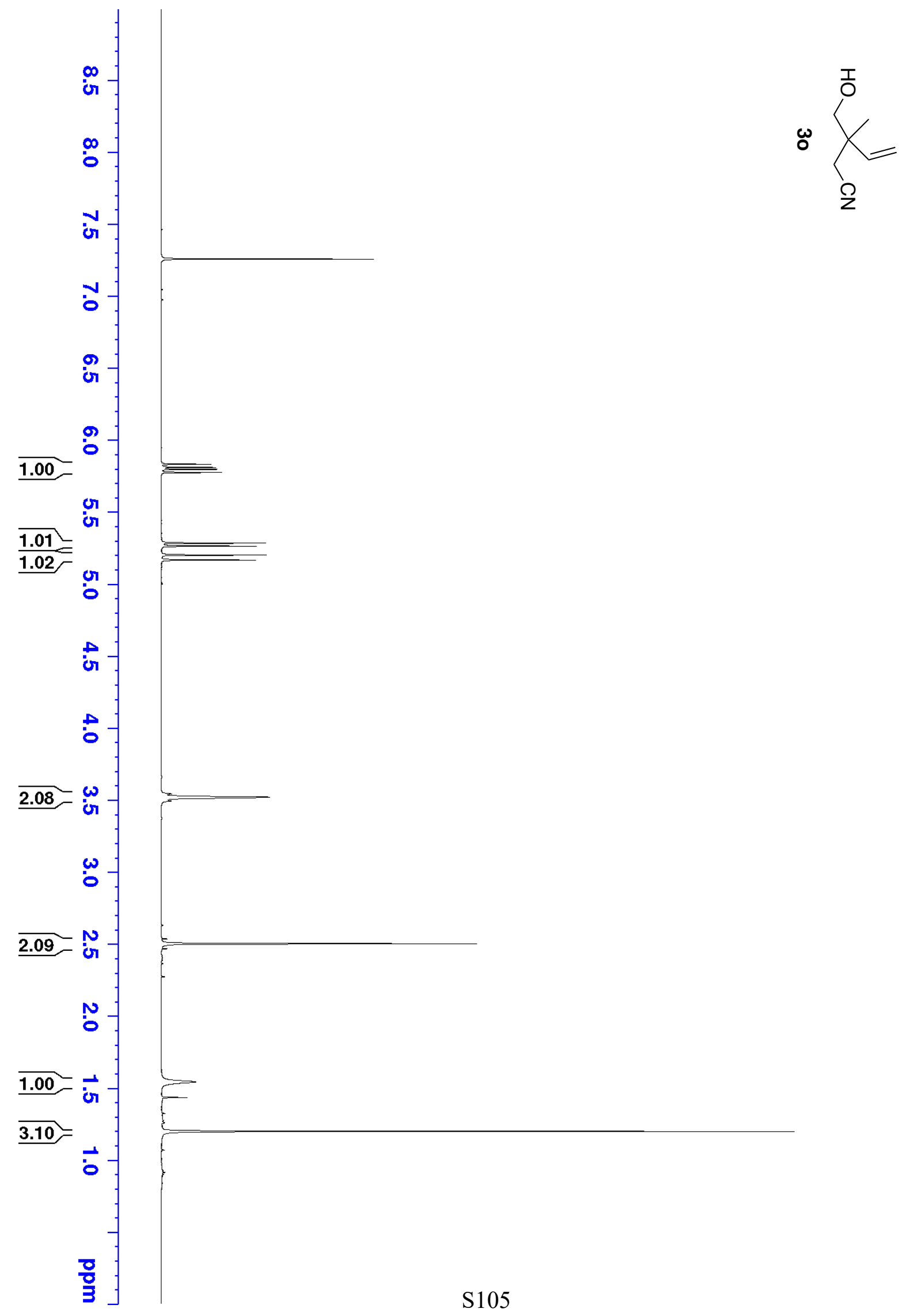




$$
\|
$$




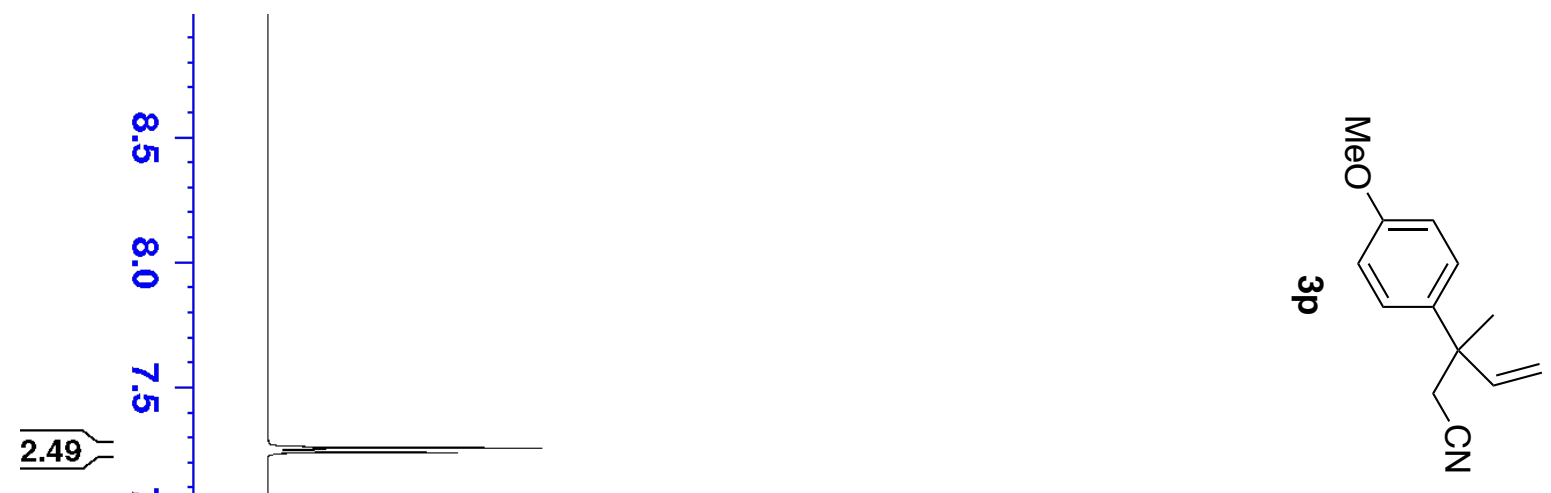




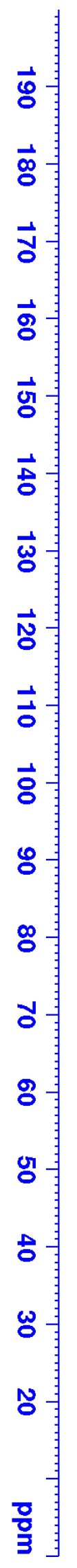
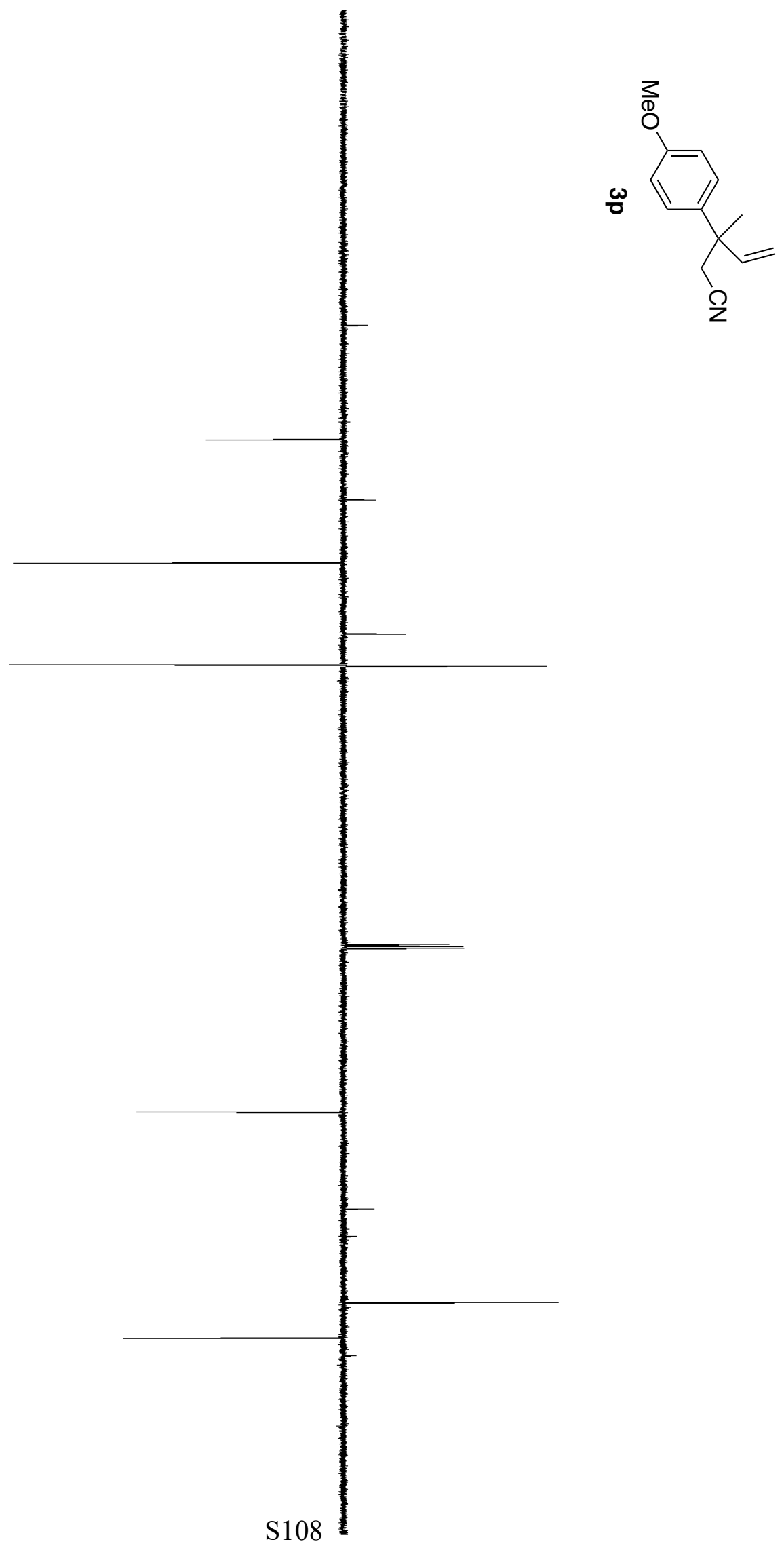

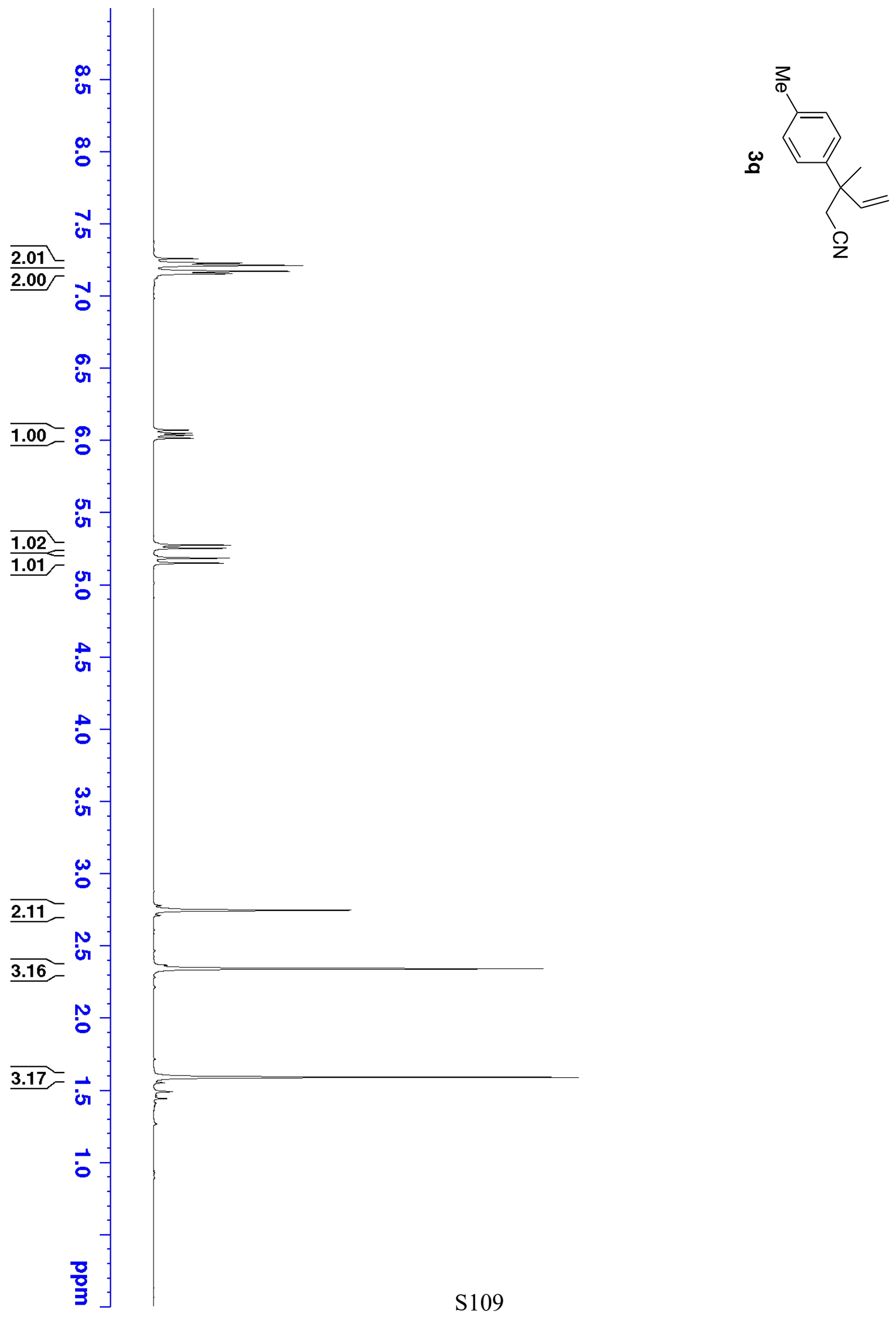


$$
\text { 怢 }
$$




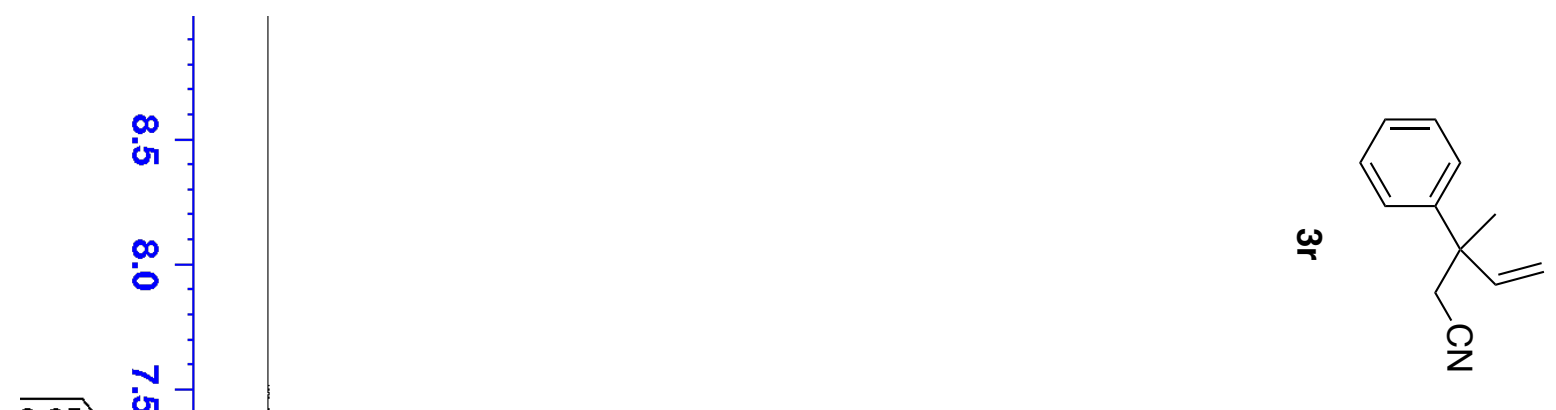

$\frac{3.95}{1.53}$
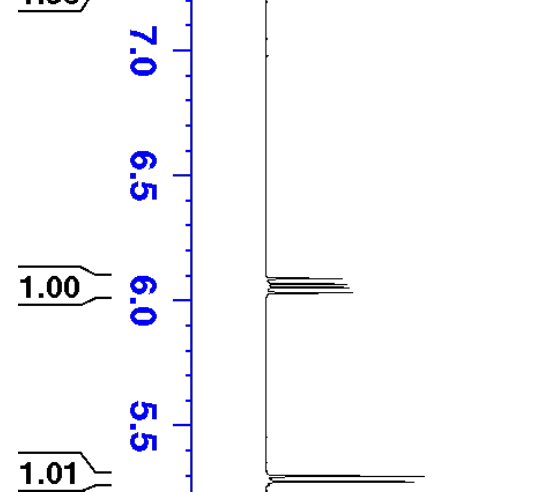

1.01

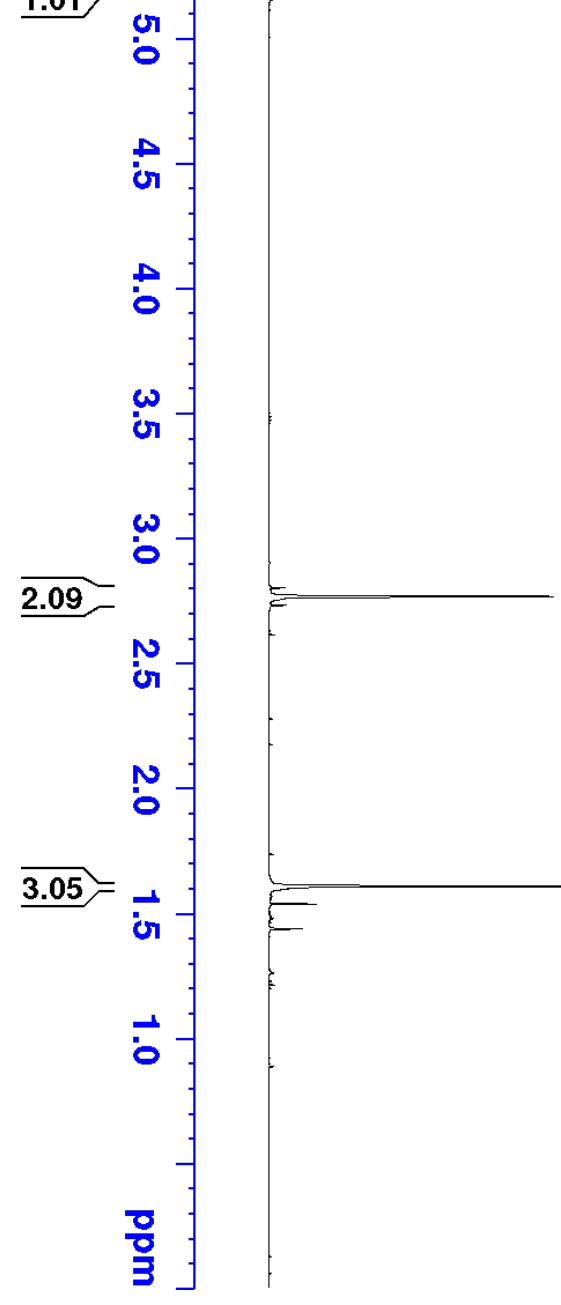




$$
\text { H' }
$$




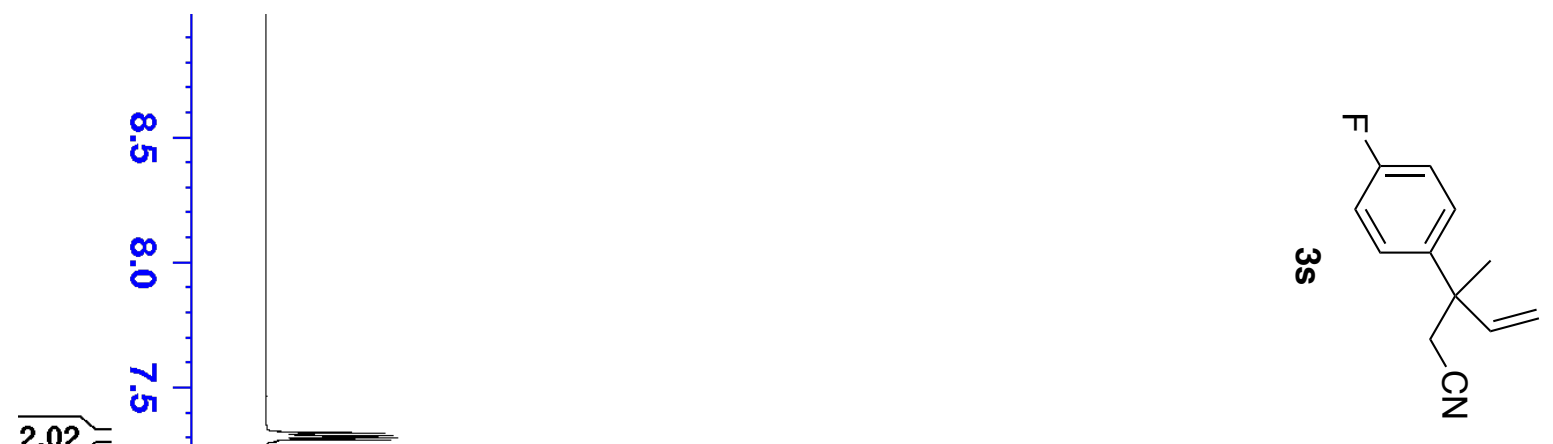

$\underline{\underline{1.99}}=\mathrm{V}$

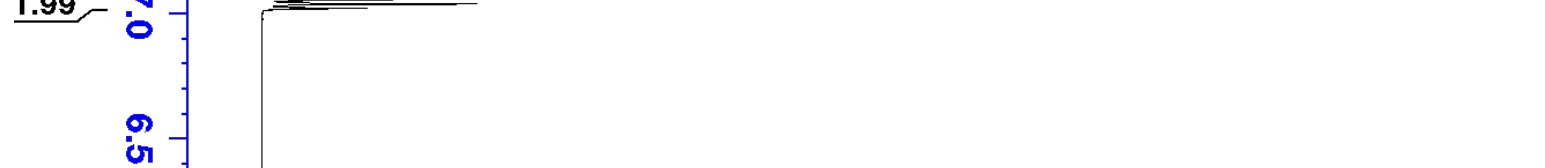

$\overline{1.00}$
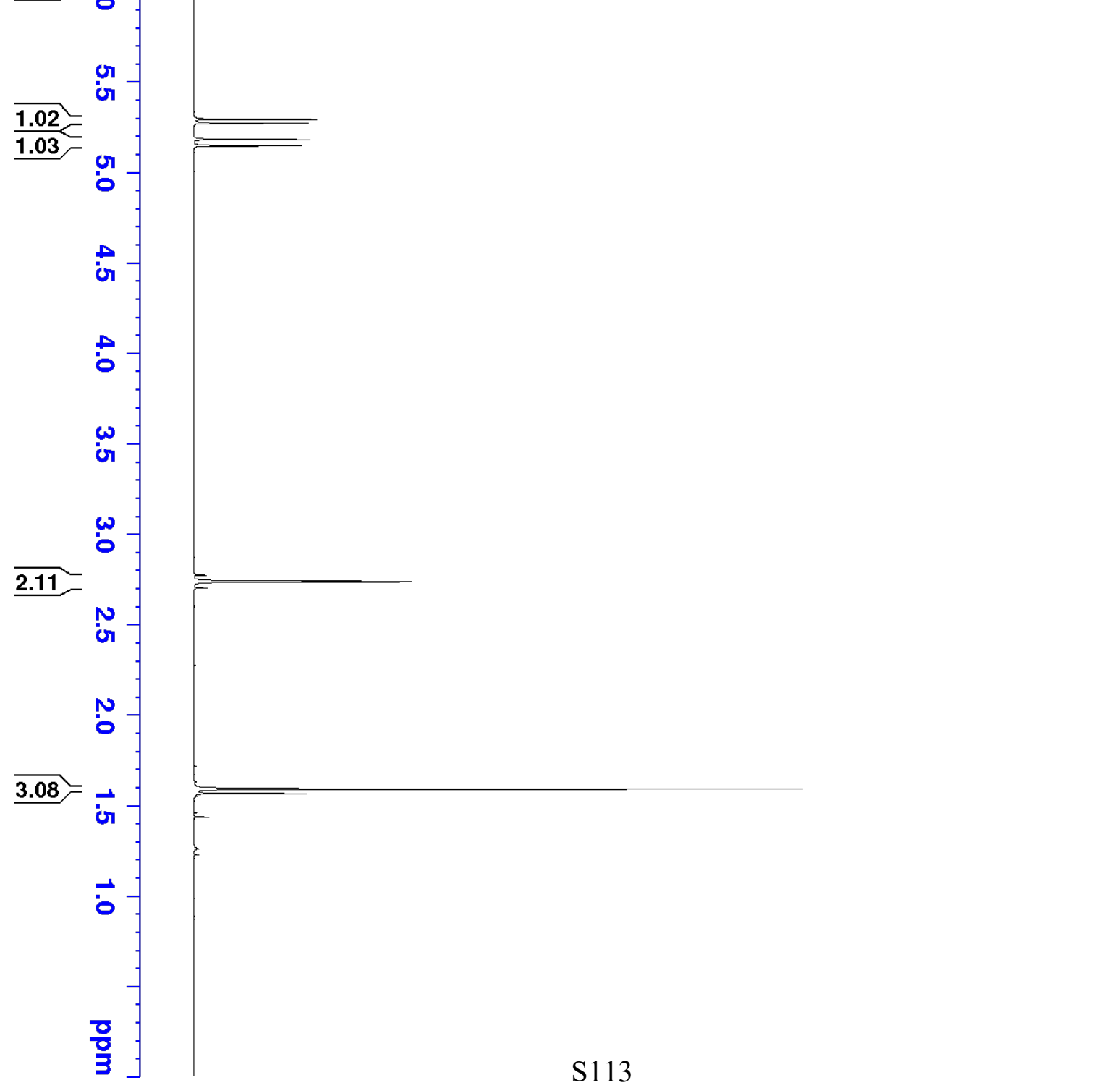


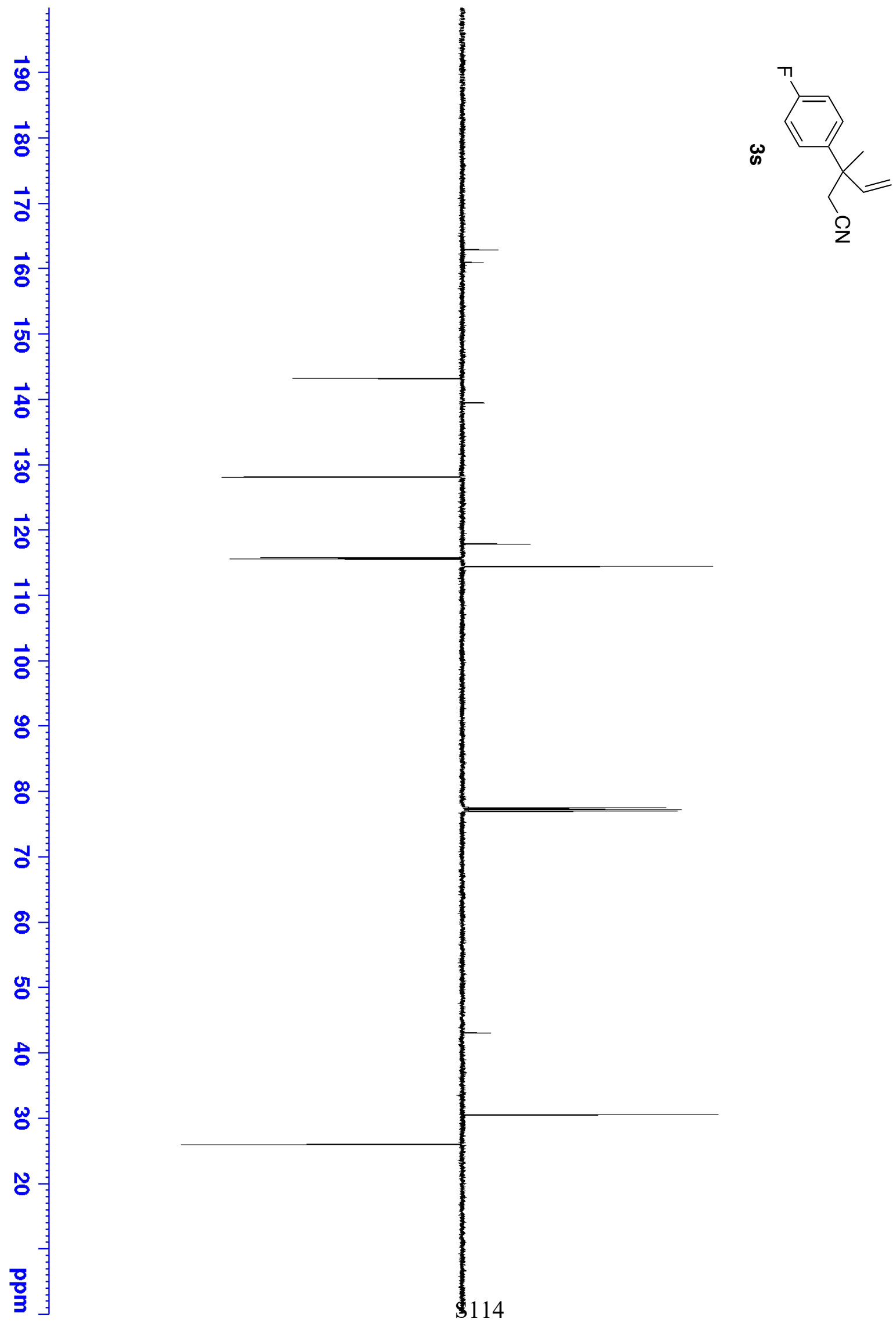




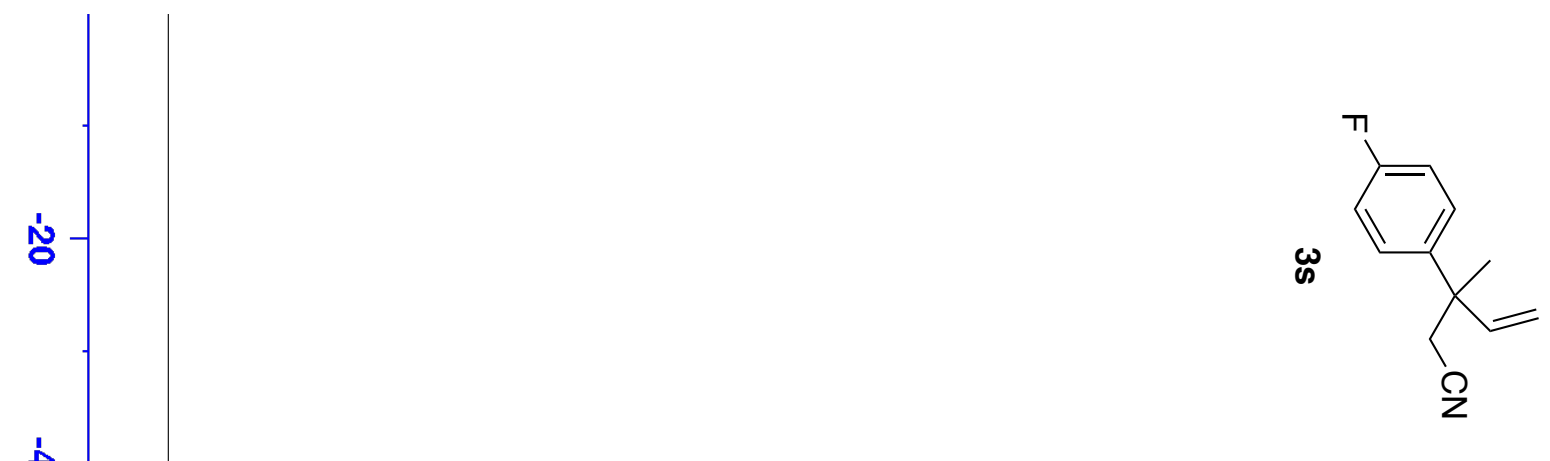

t

s

\&

$\dot{b}$

$\dot{\vec{s}}$

妾

$\dot{\vec{g}}$

$\dot{\overrightarrow{\mathrm{g}}}$

믈 

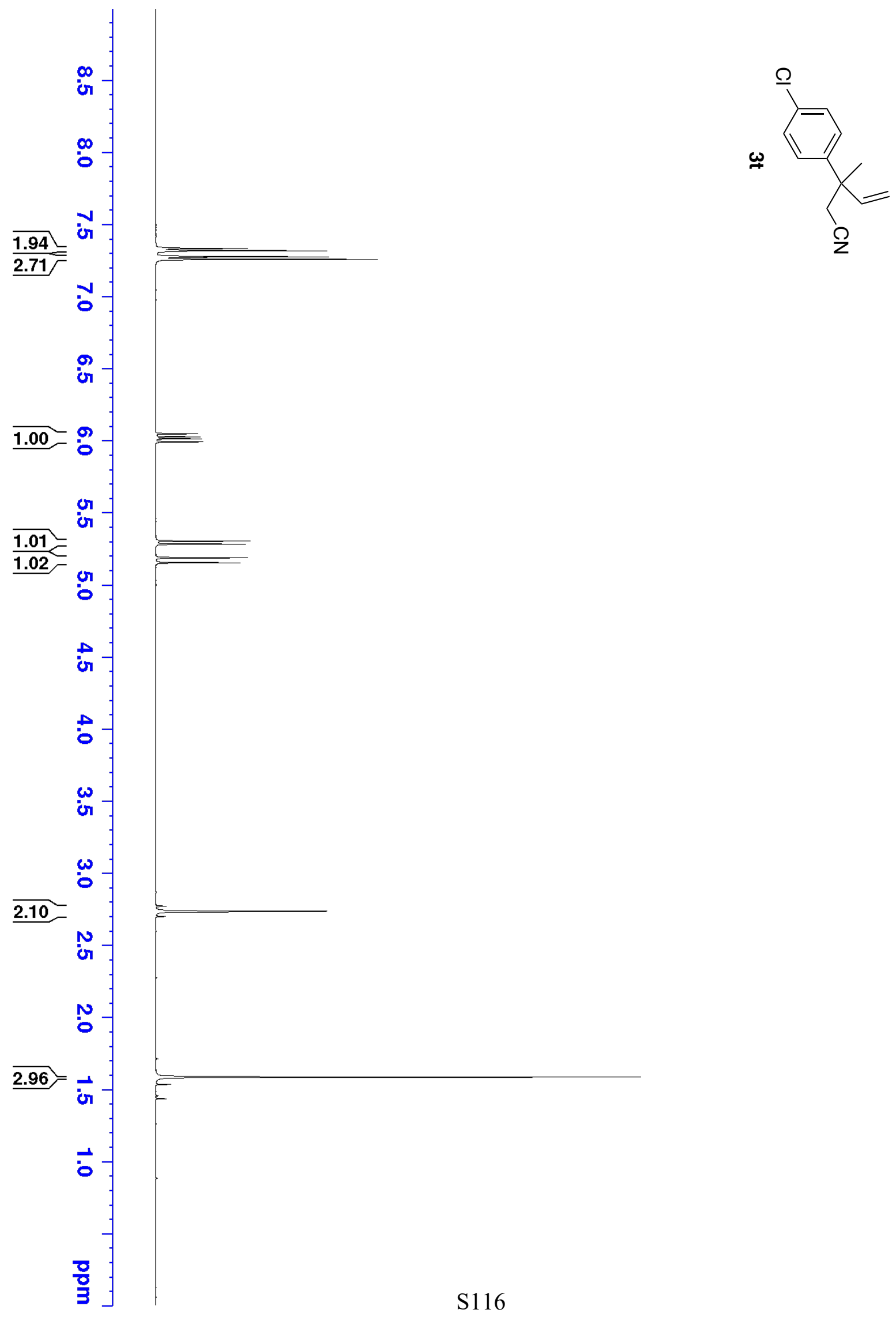


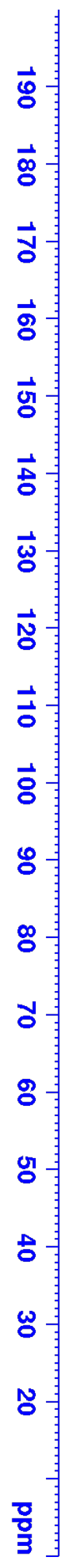

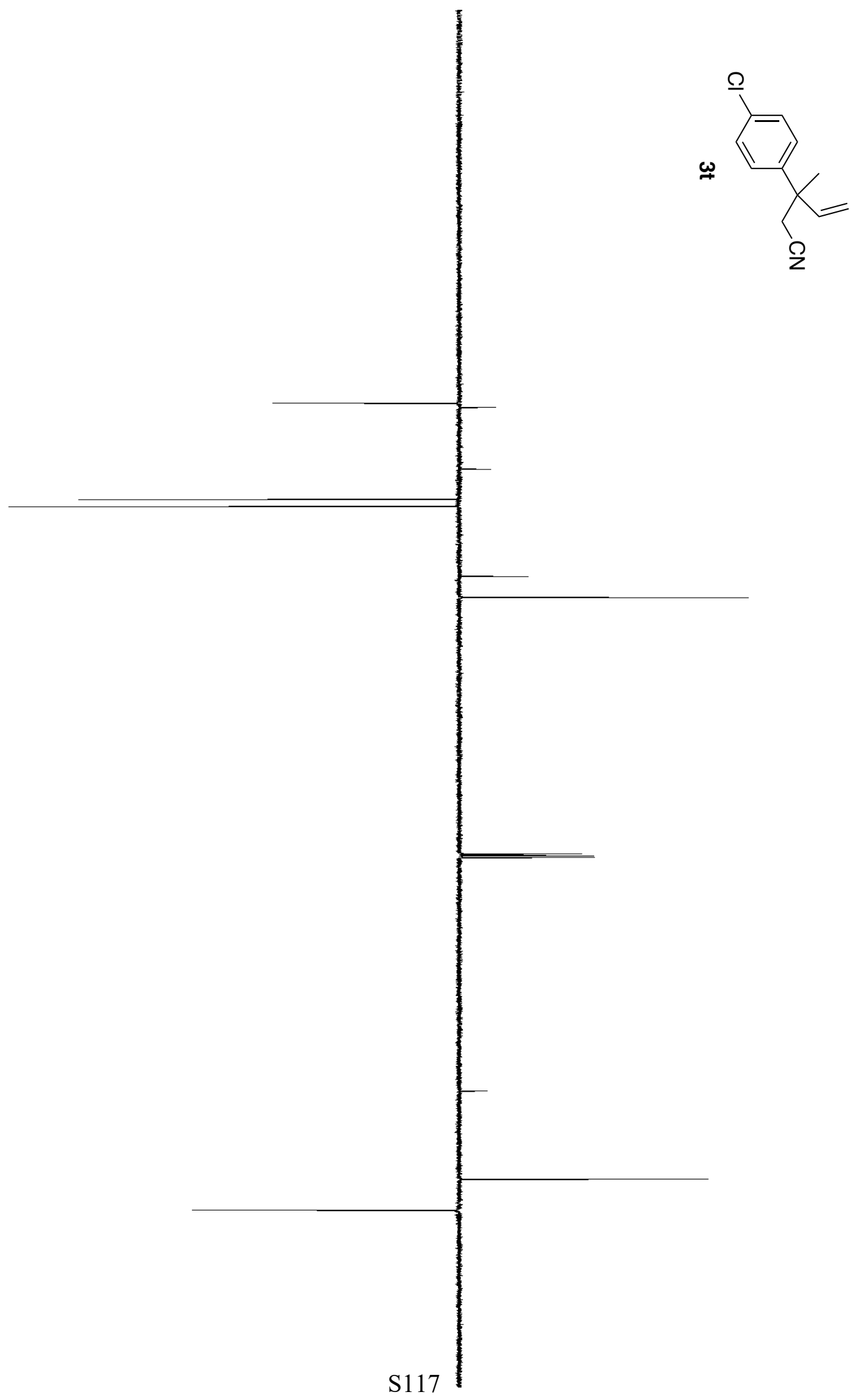




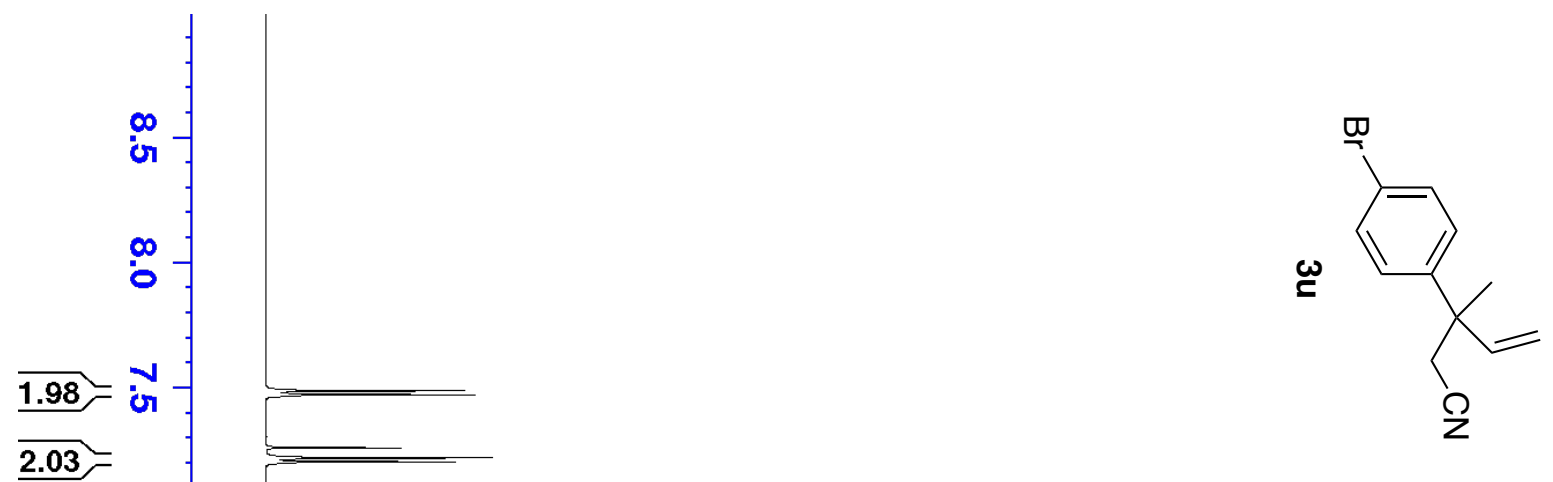




$$
\text { He }
$$




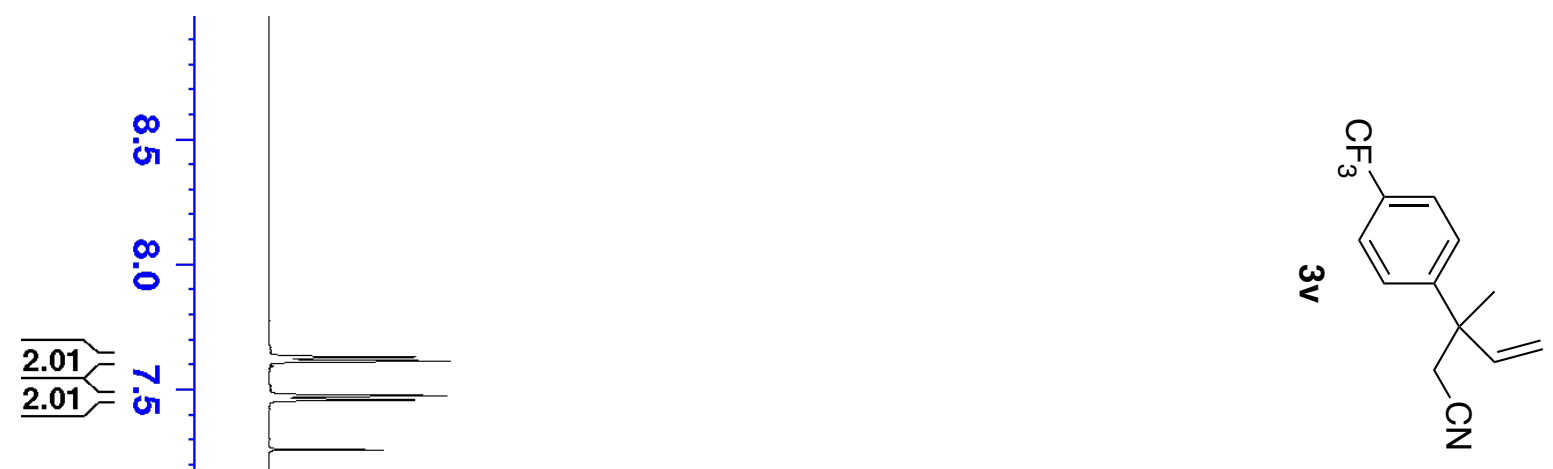




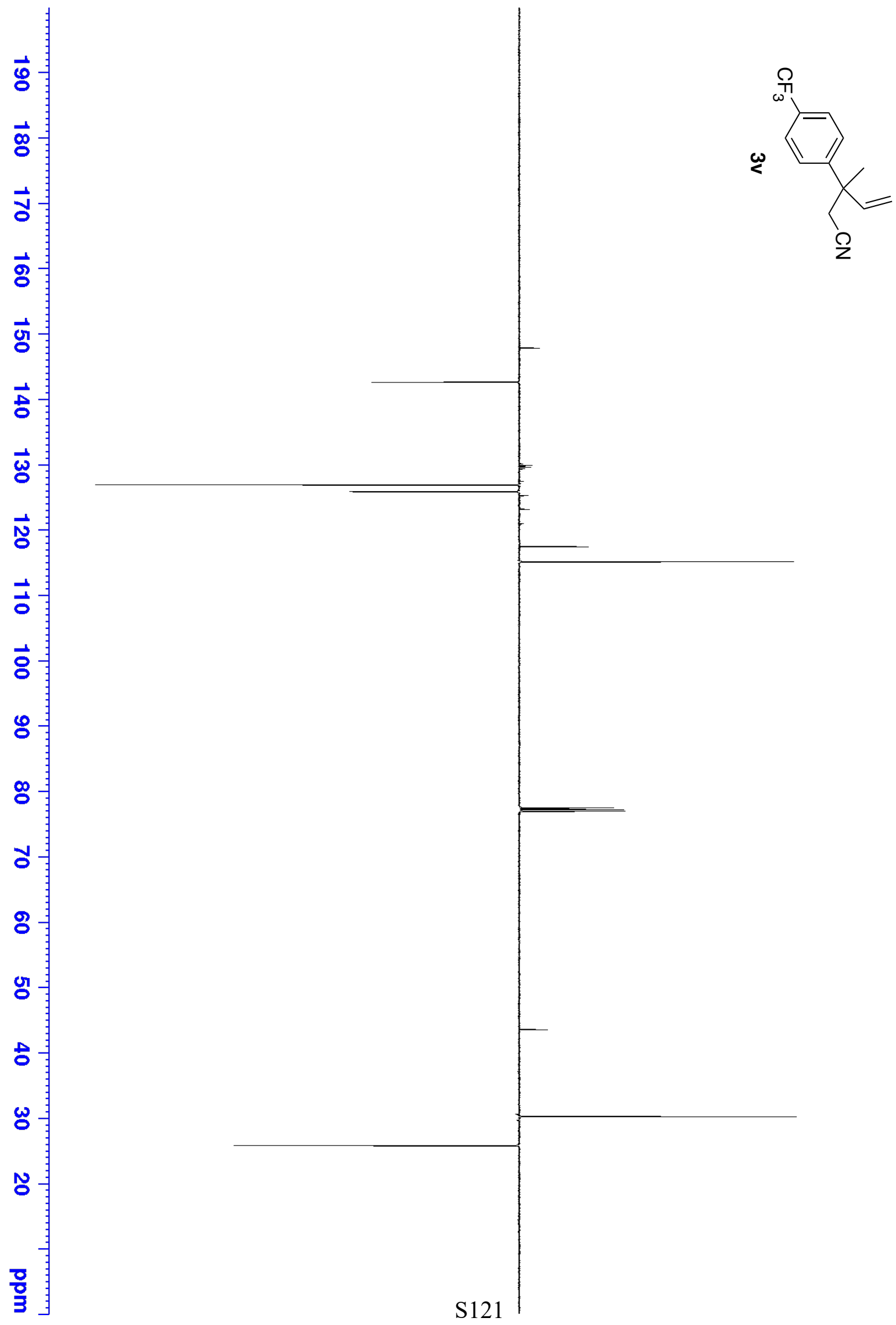




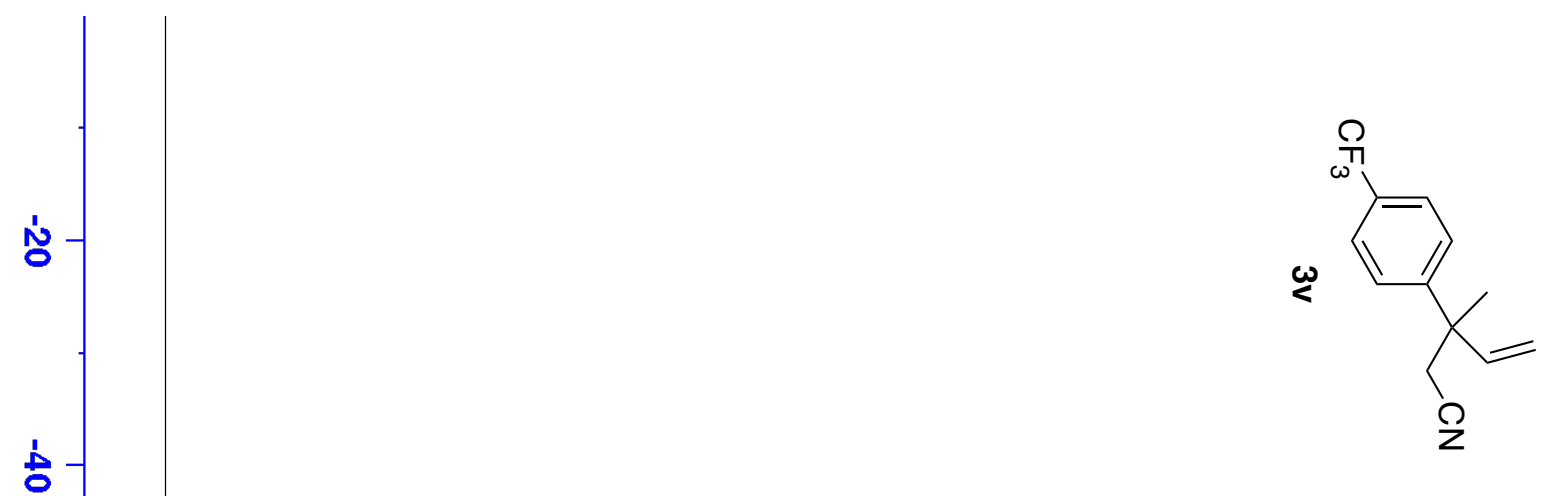

ஜ

$\dot{\overrightarrow{8}}$

$\dot{\vec{N}}$

$\dot{\vec{t}}$

$\dot{\overrightarrow{9}}$

㐫

흠

S122 


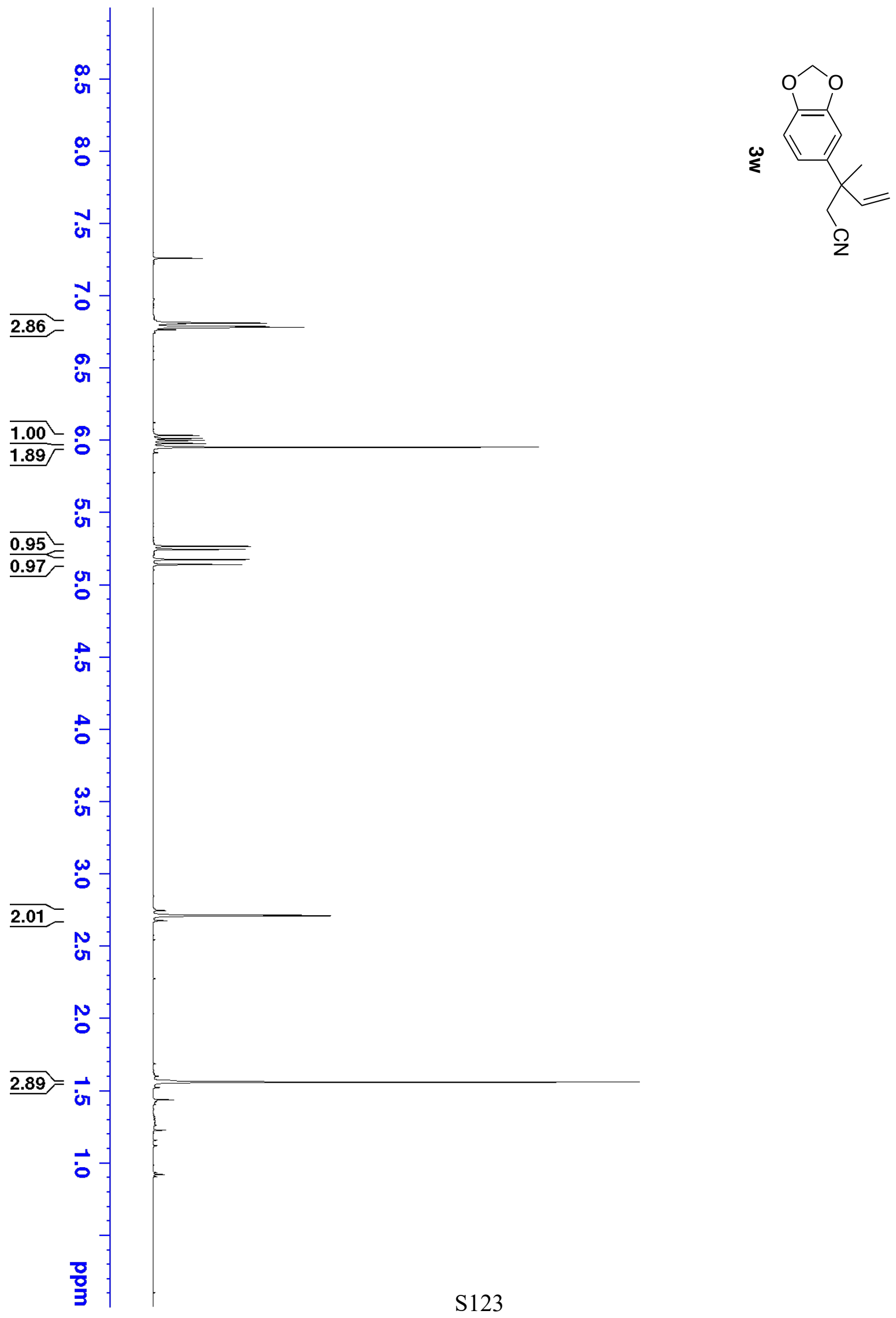




$$
H^{*}
$$




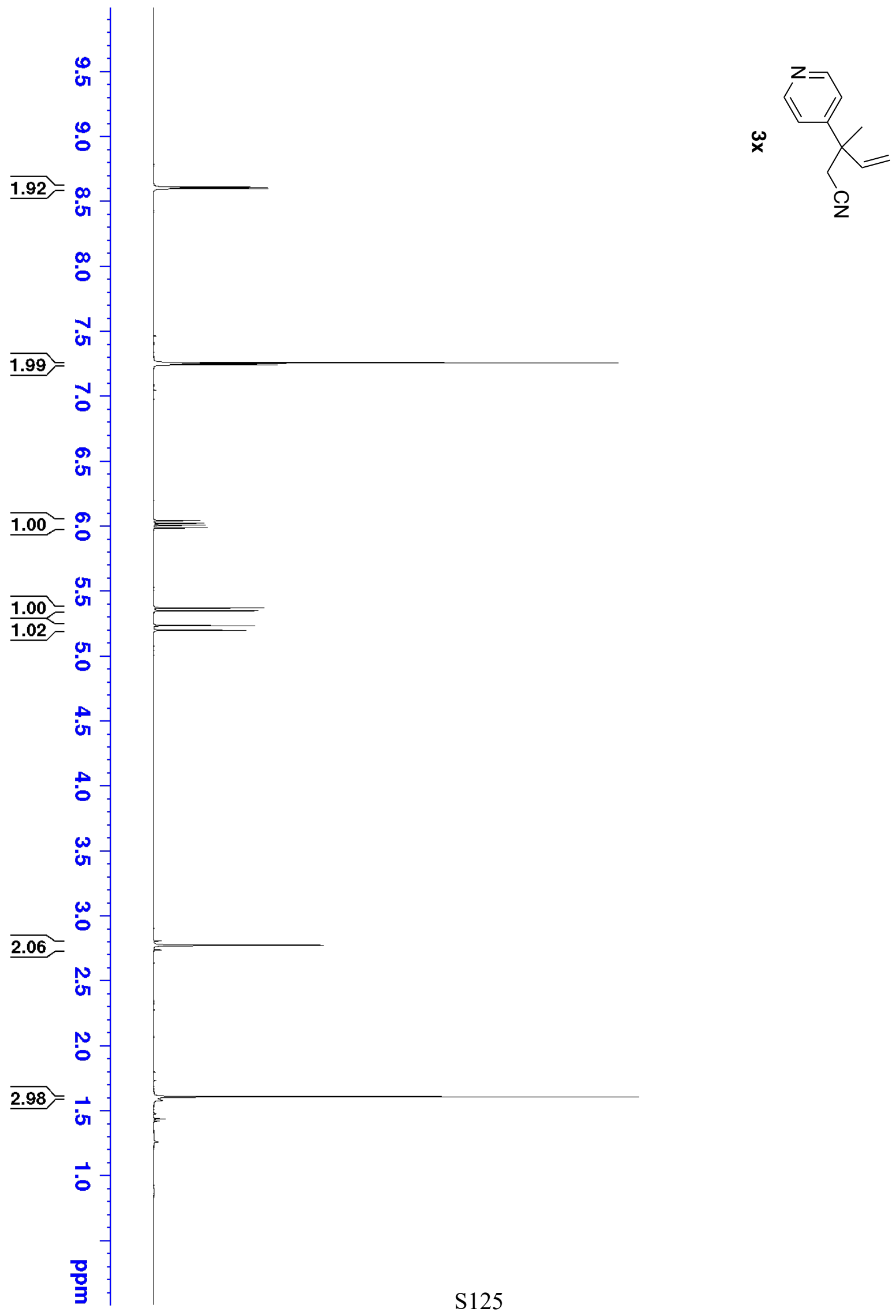




$$
\text { |l” }
$$




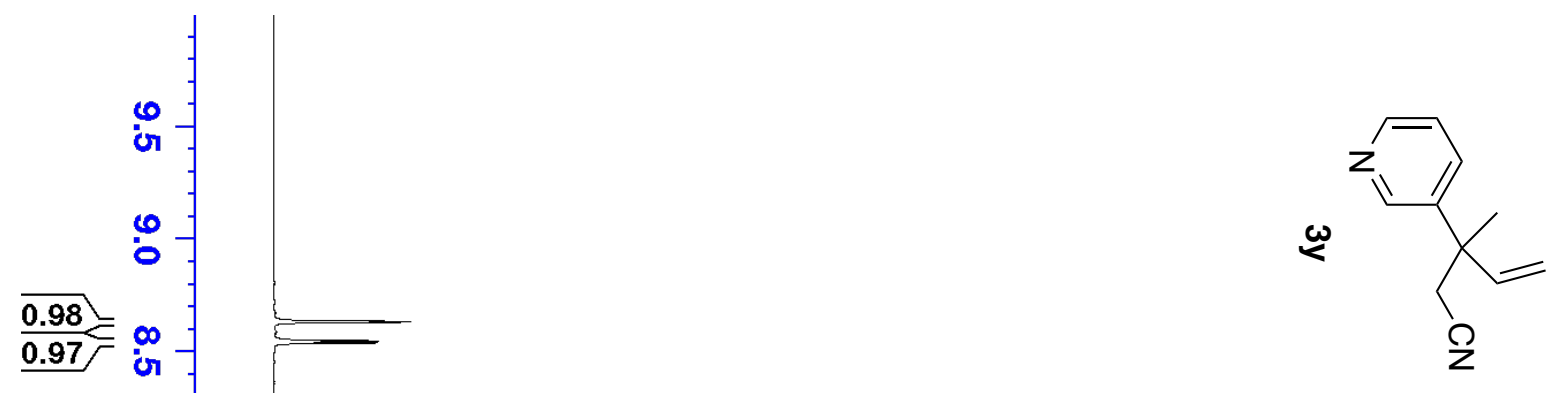




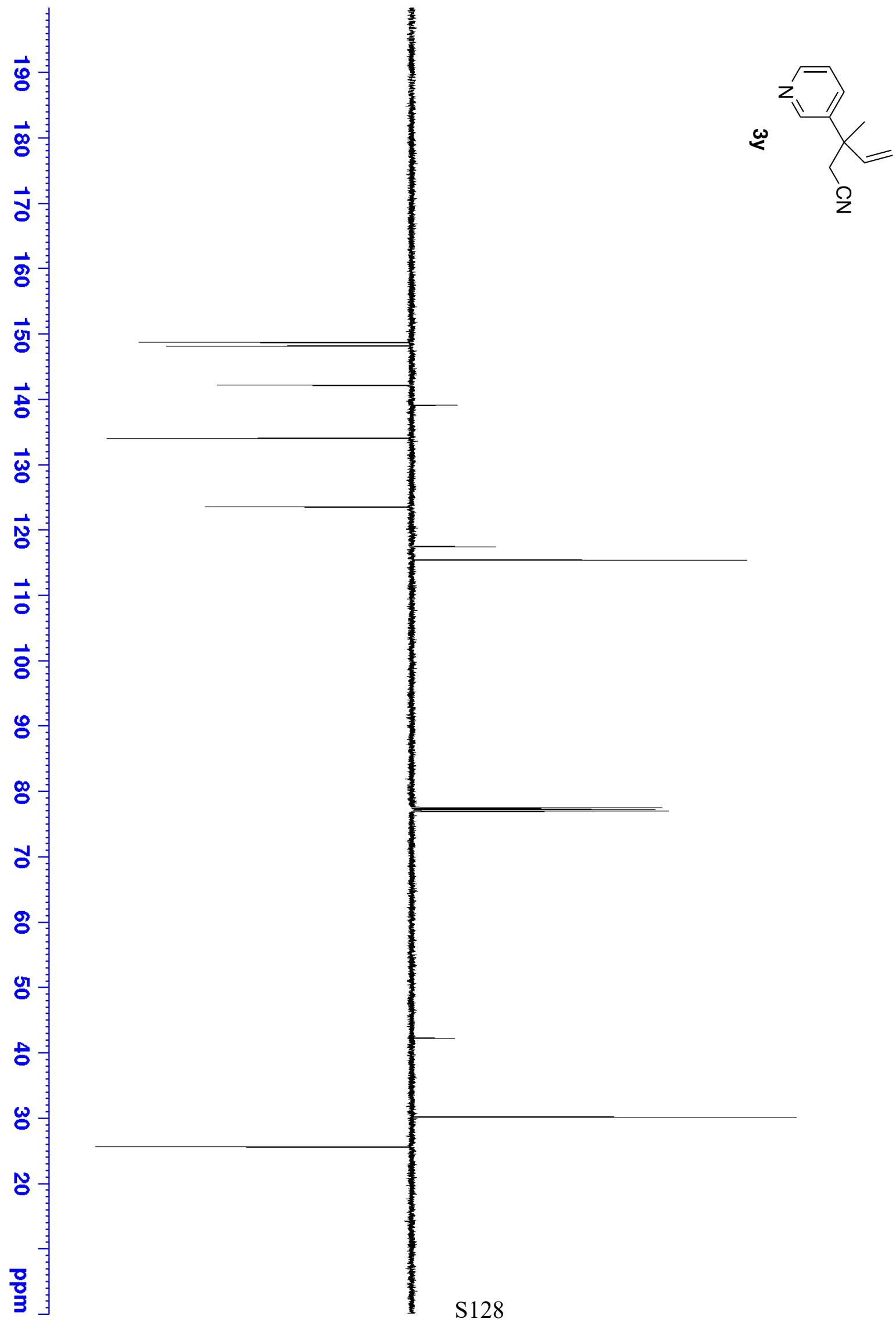



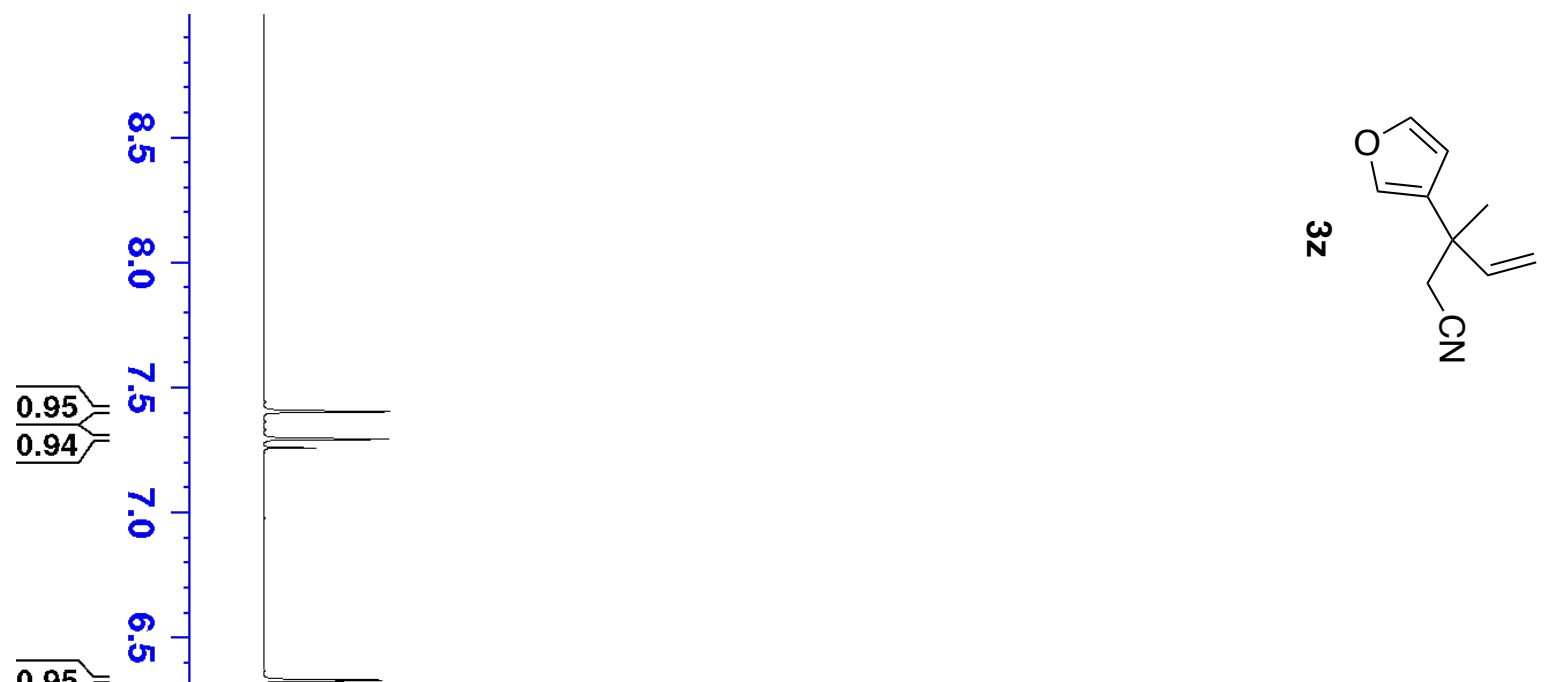

$\underline{1.00}$

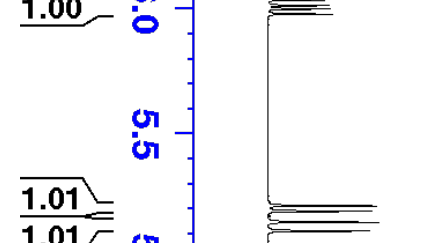

$1.01 \%$ or
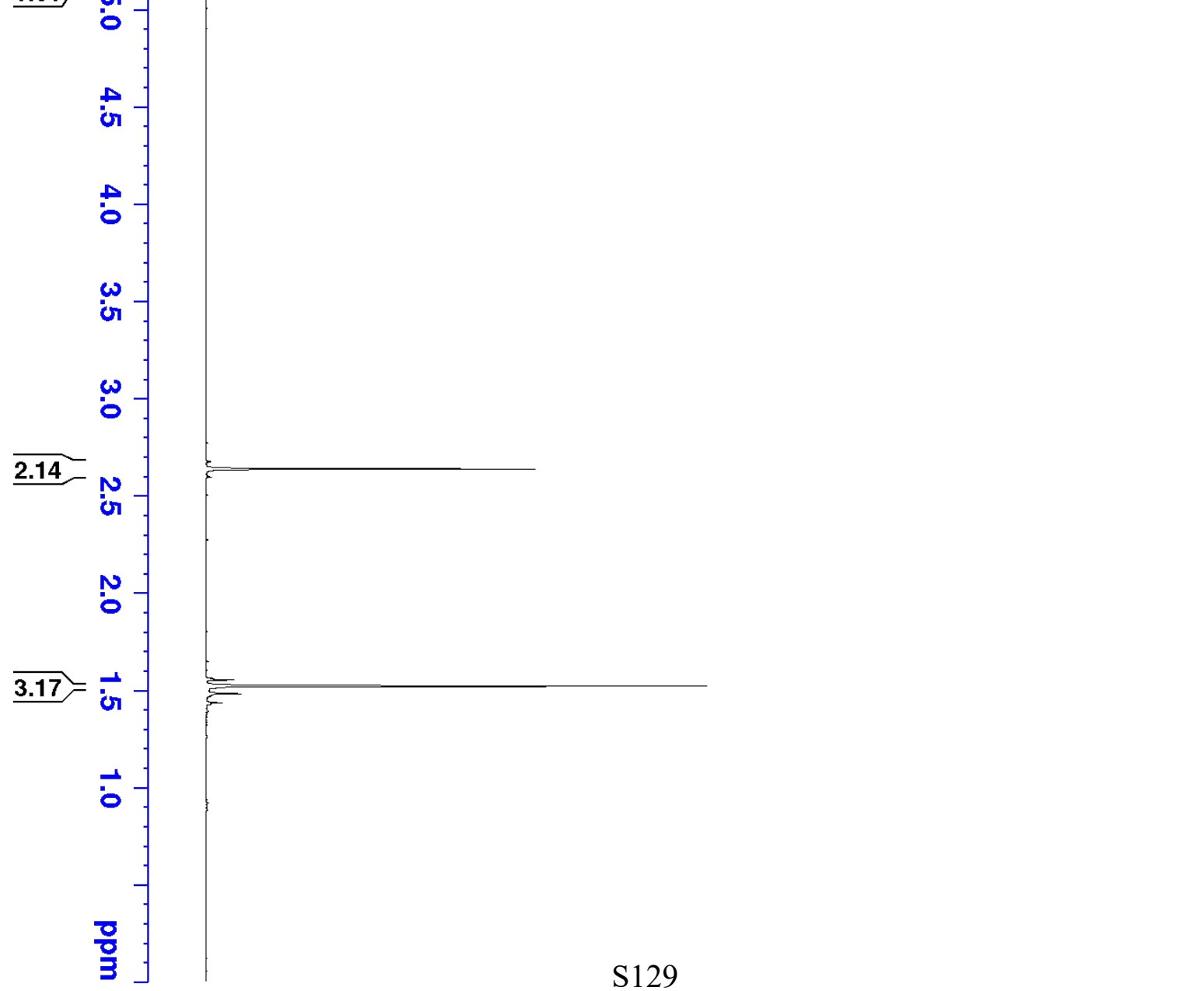

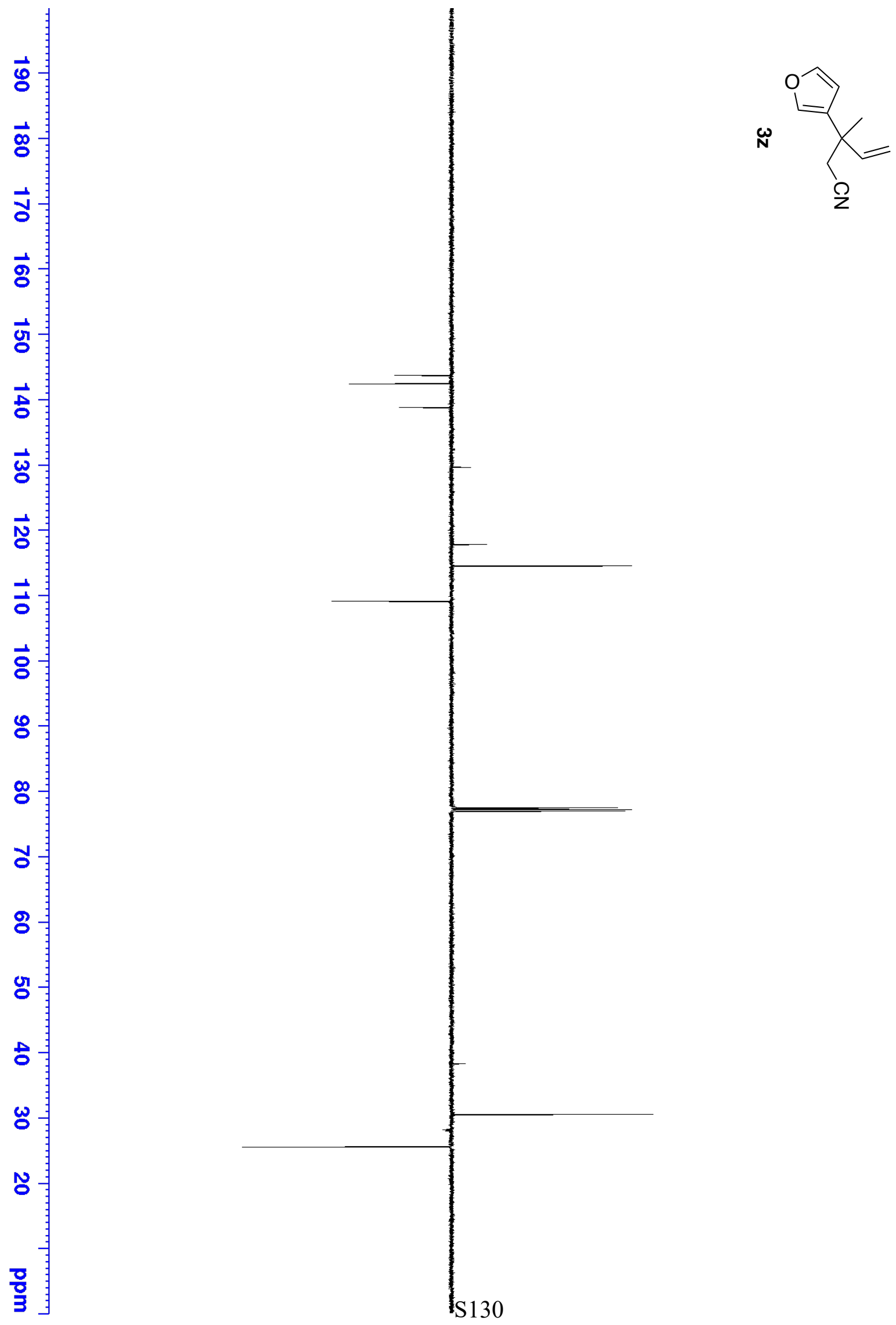

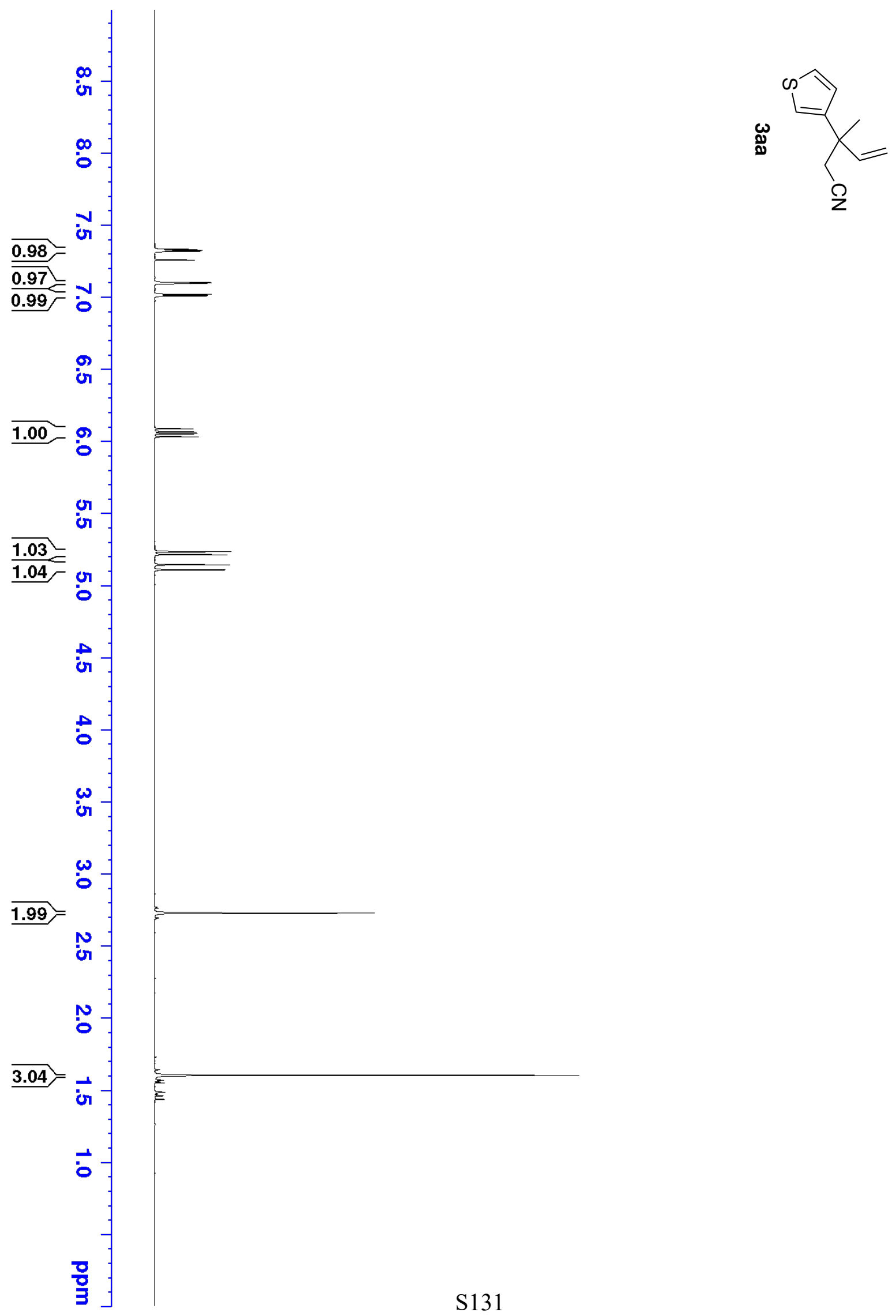


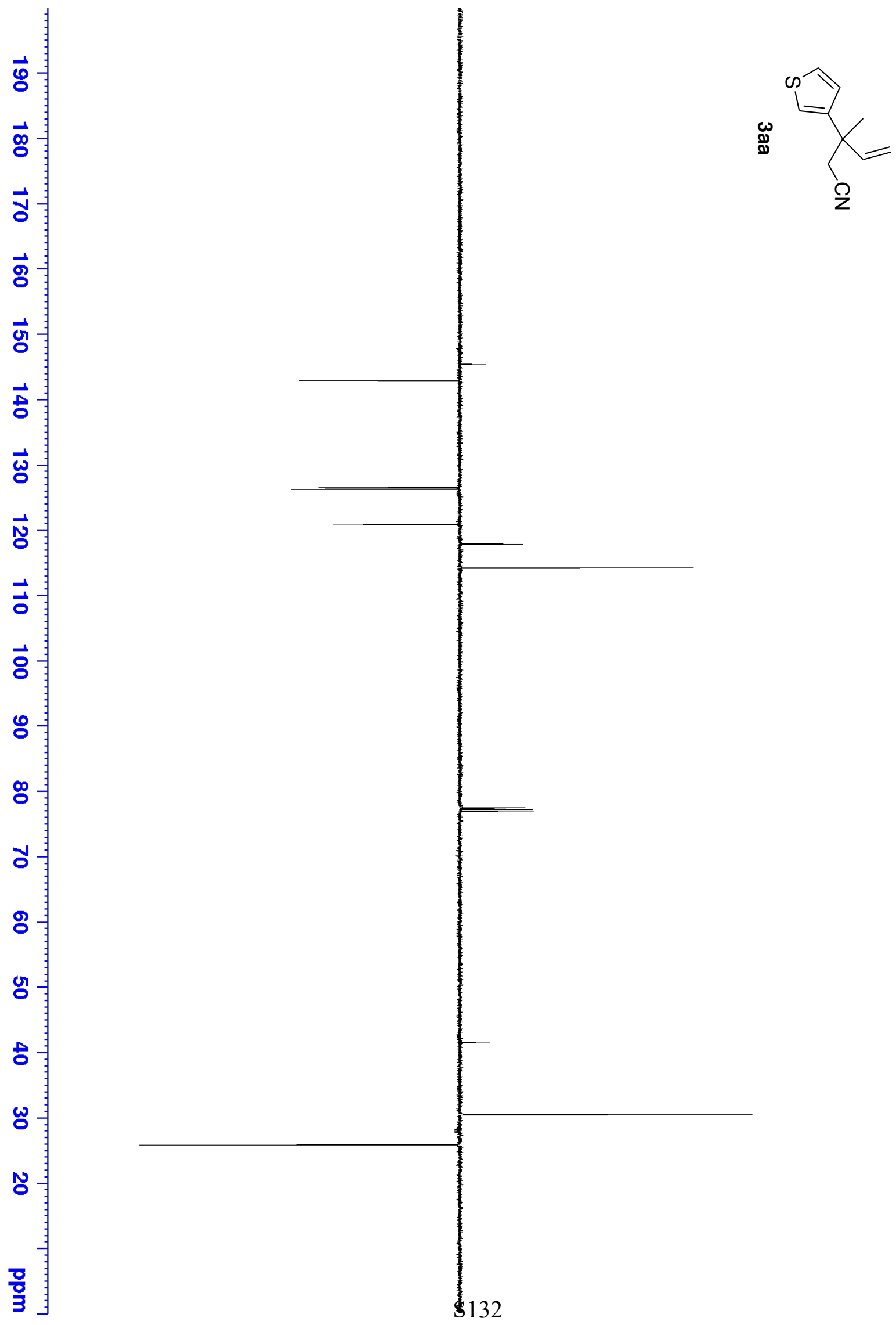




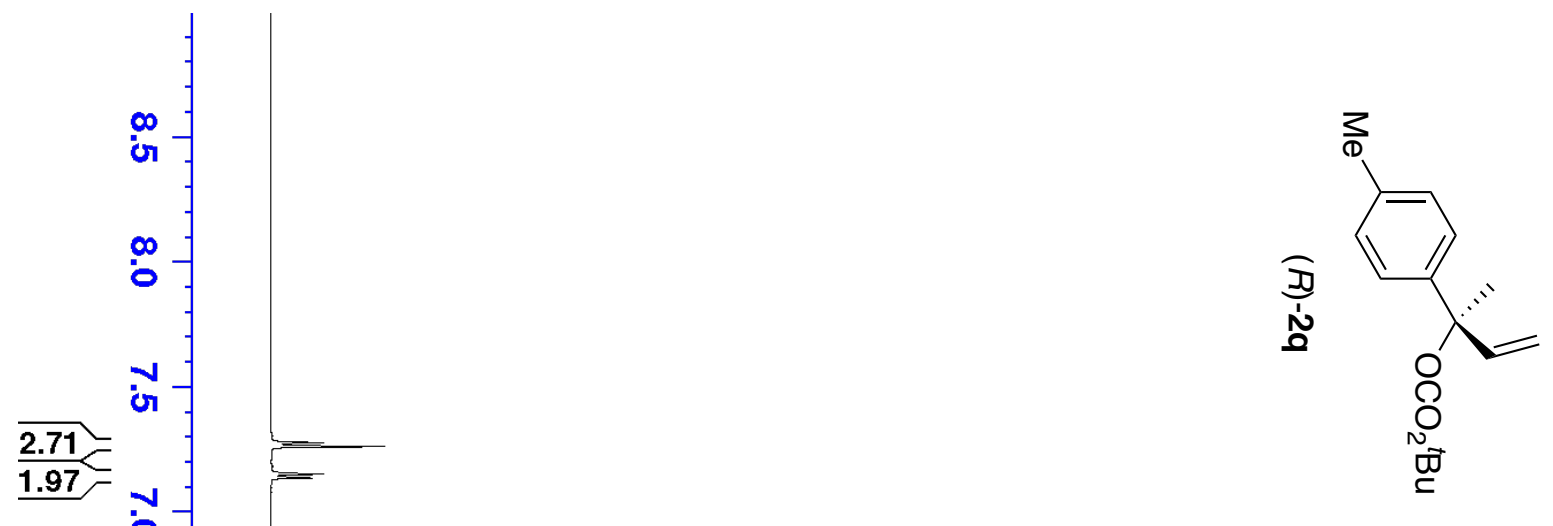



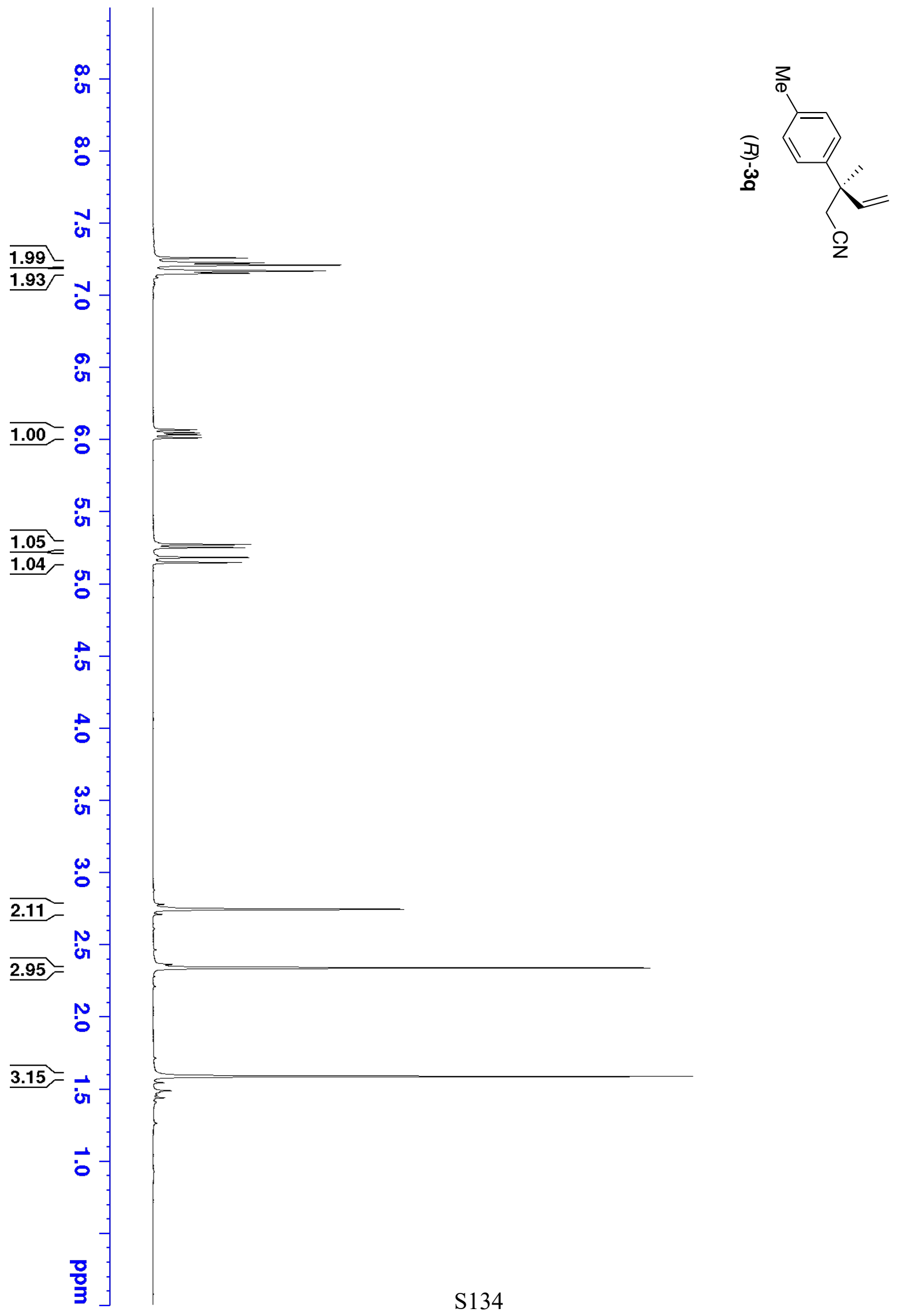


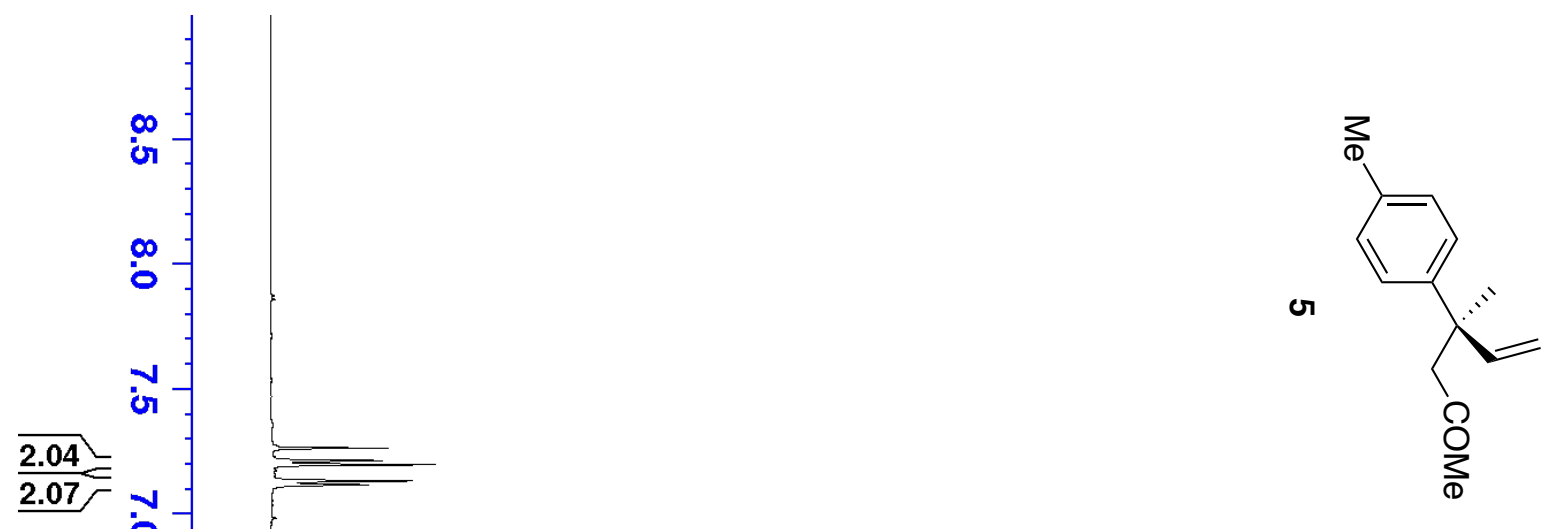




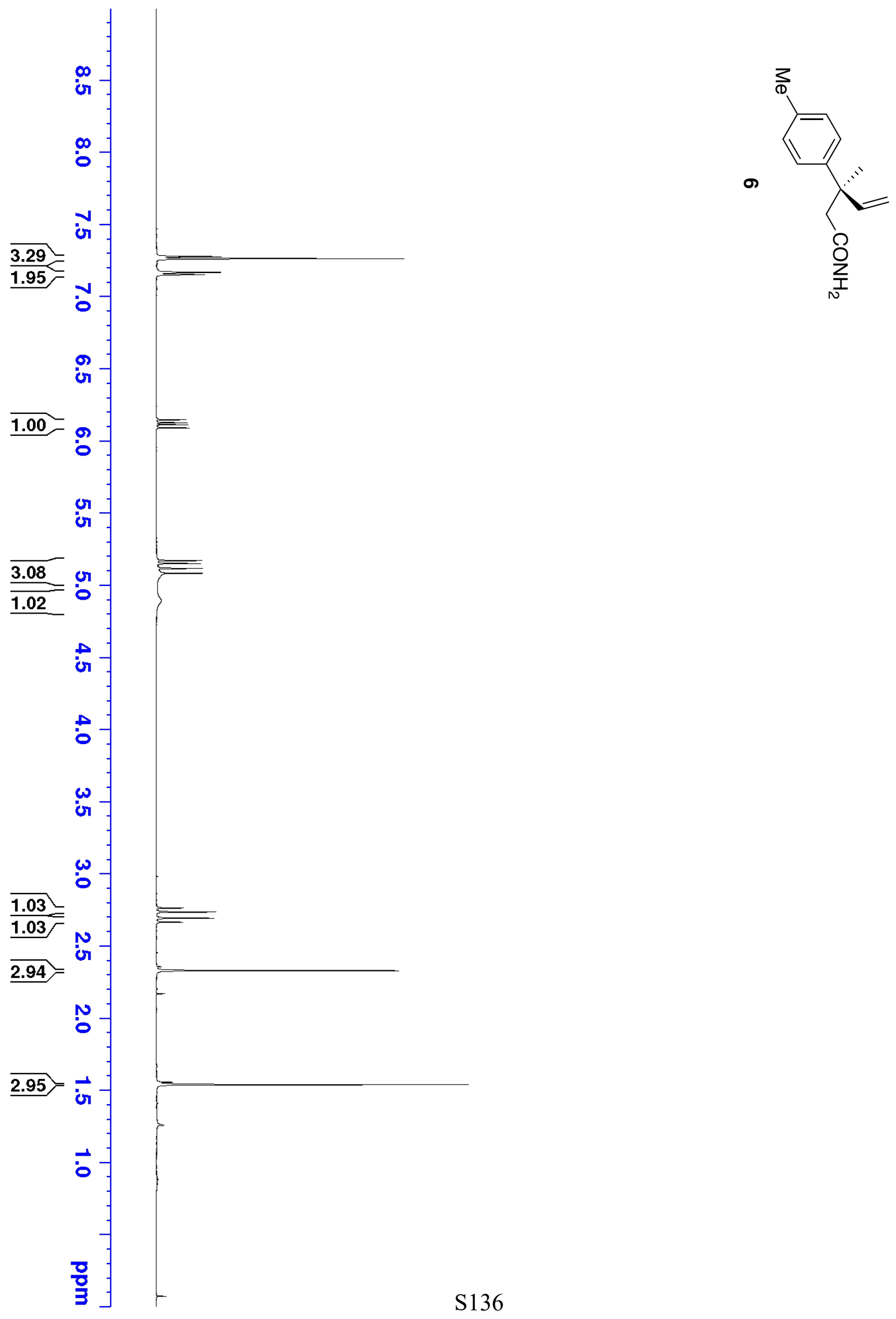




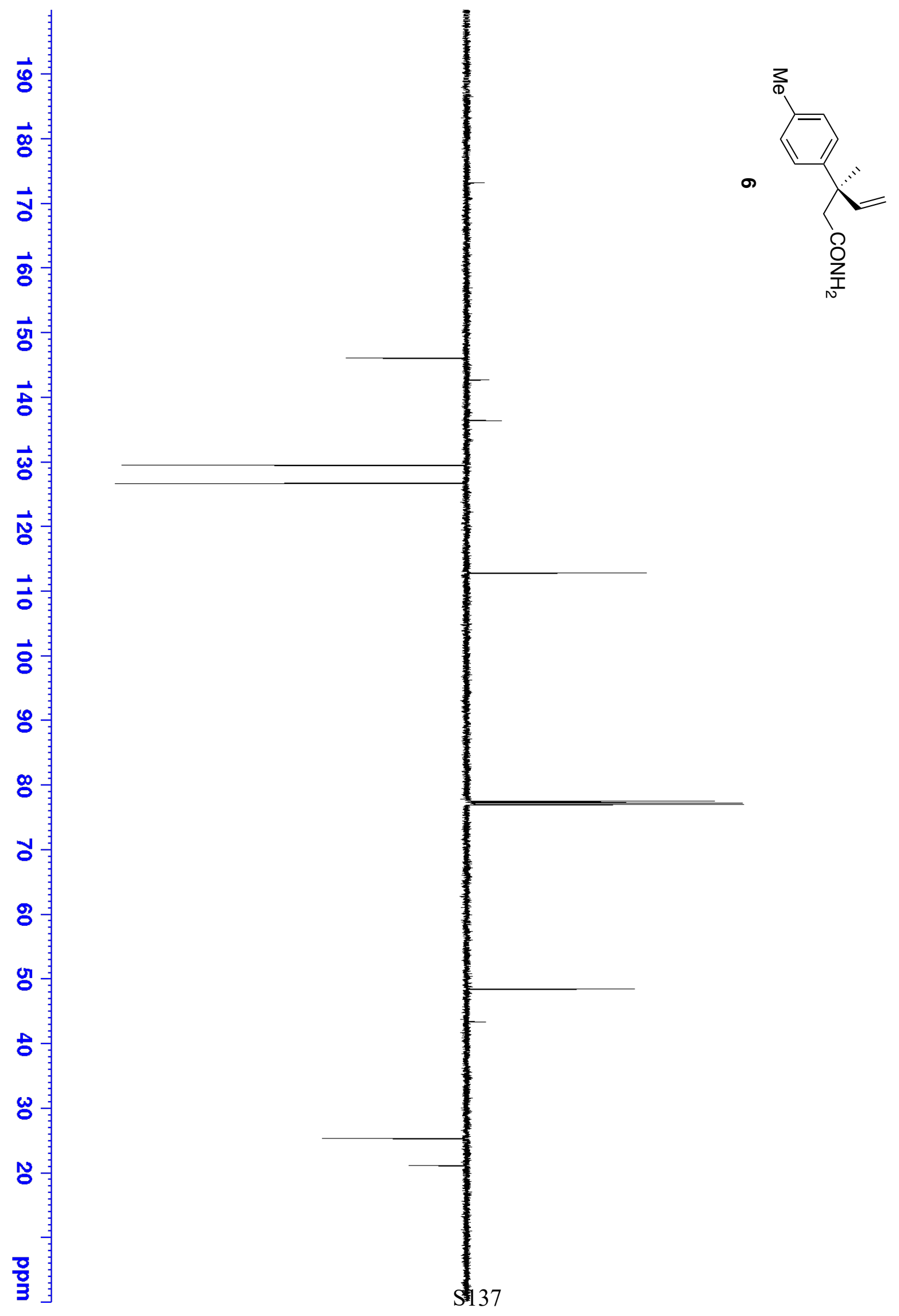




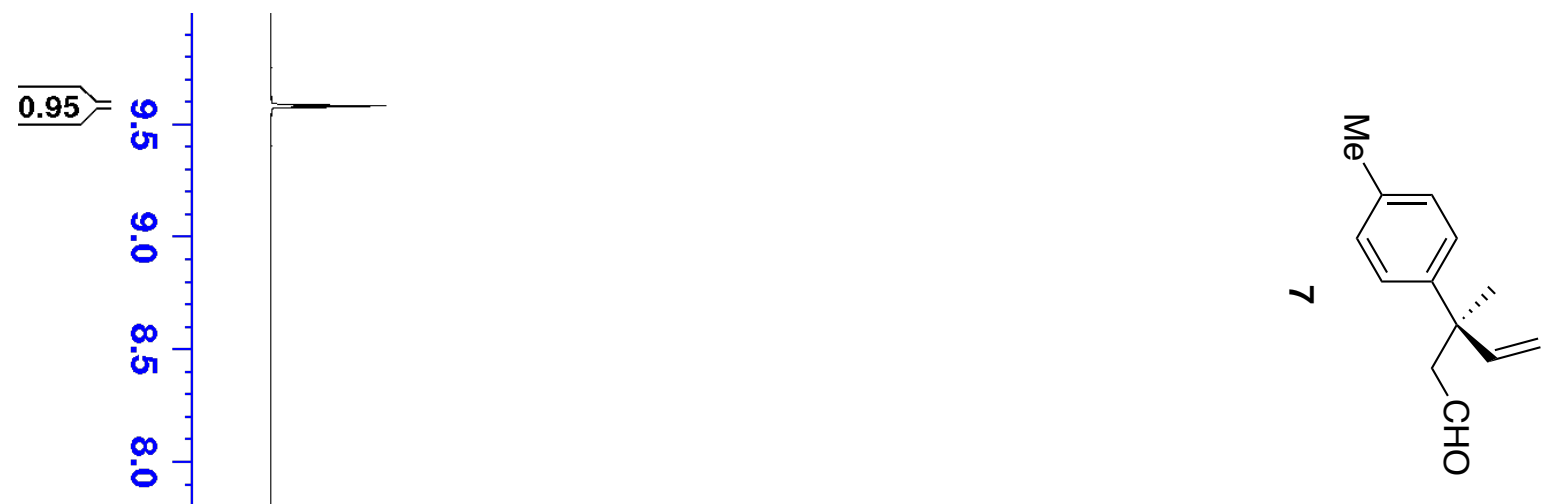




$$
H^{\prime}
$$




\section{HPLC Chromatograms}

3,7-Dimethylocta-1,6-dien-3-yl methyl carbonate (2f)

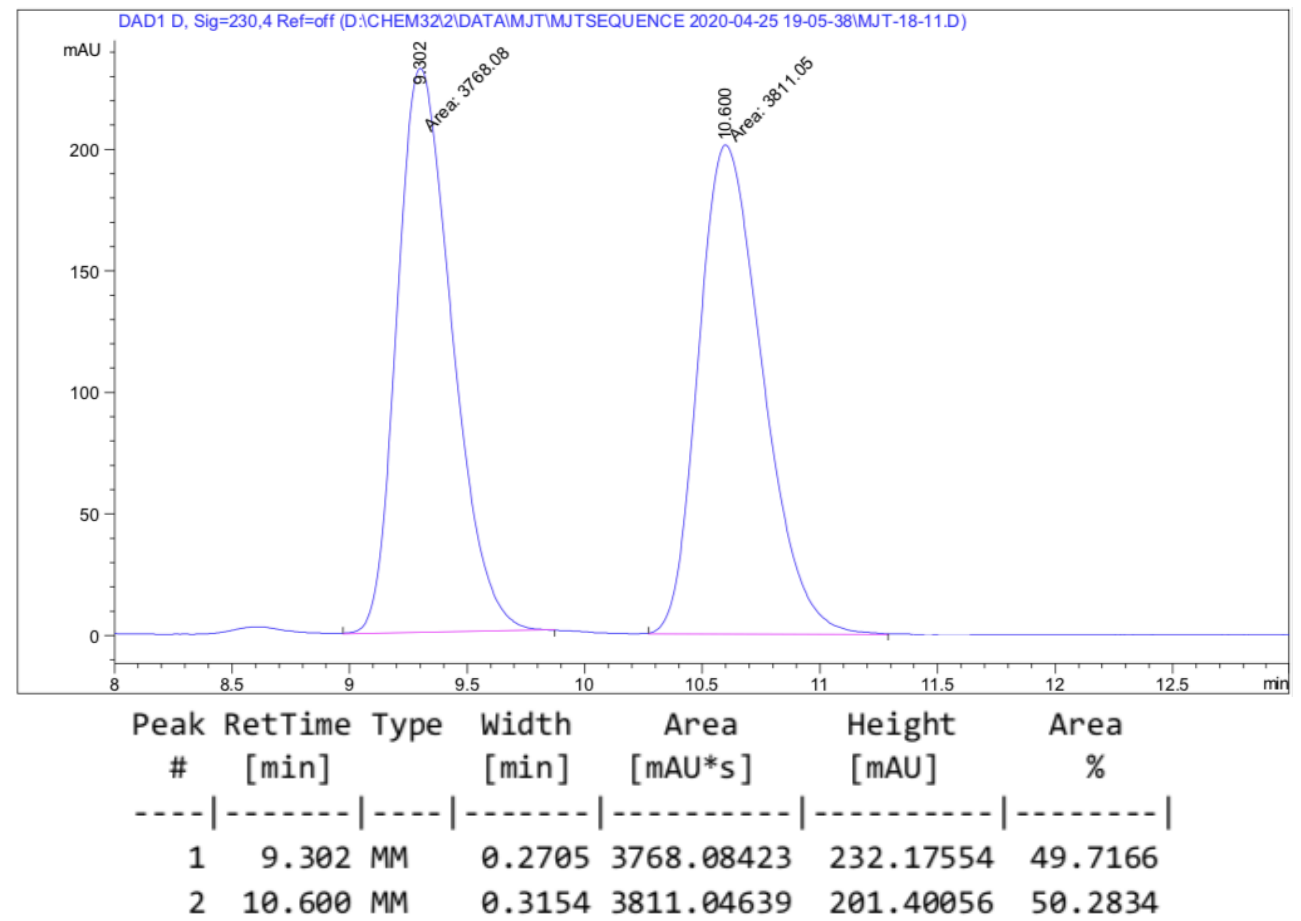

(R)-3,7-Dimethylocta-1,6-dien-3-yl methyl carbonate $((R)-2 \mathrm{f})$

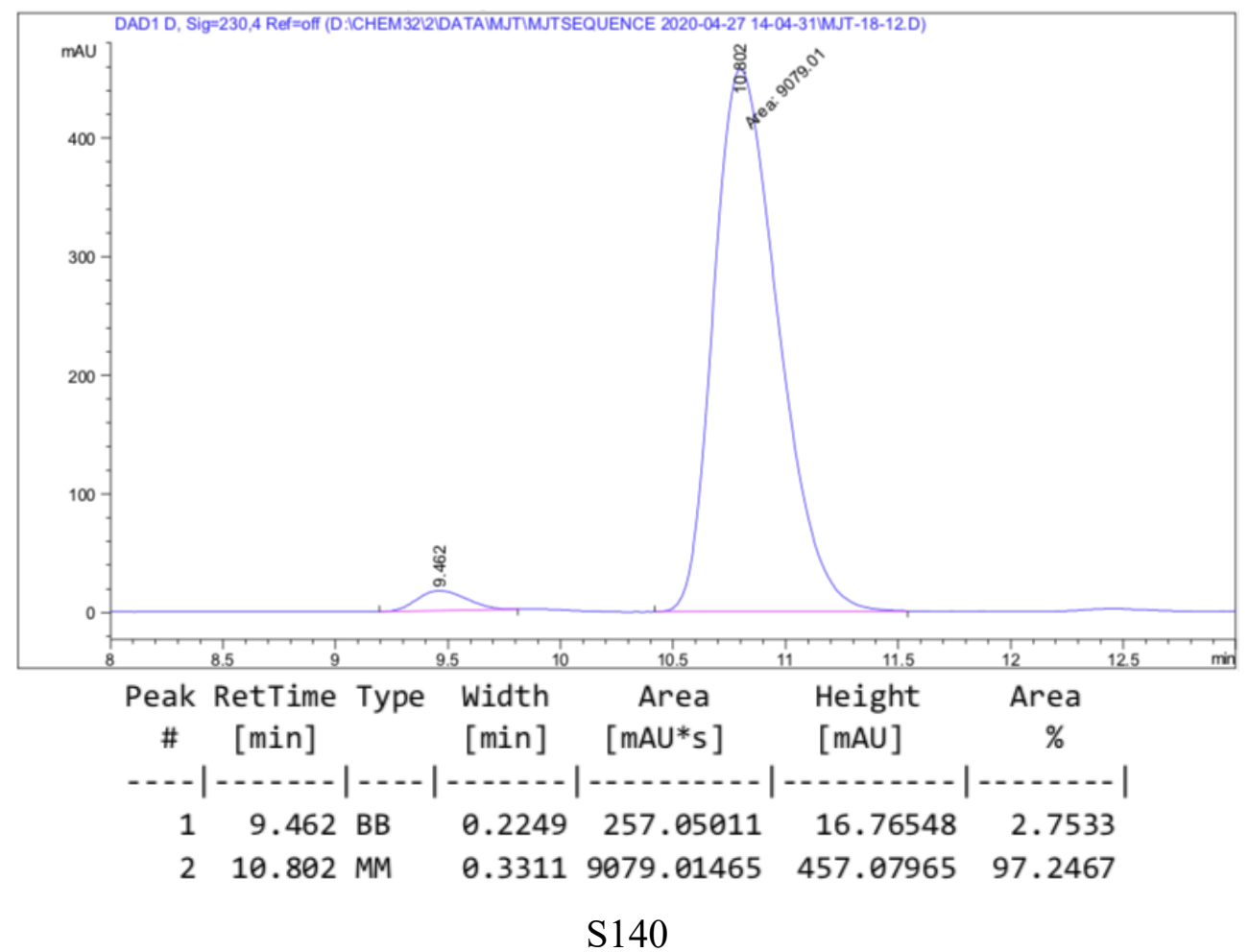


3,7-Dimethyl-3-vinyloct-6-enenitrile (3f)

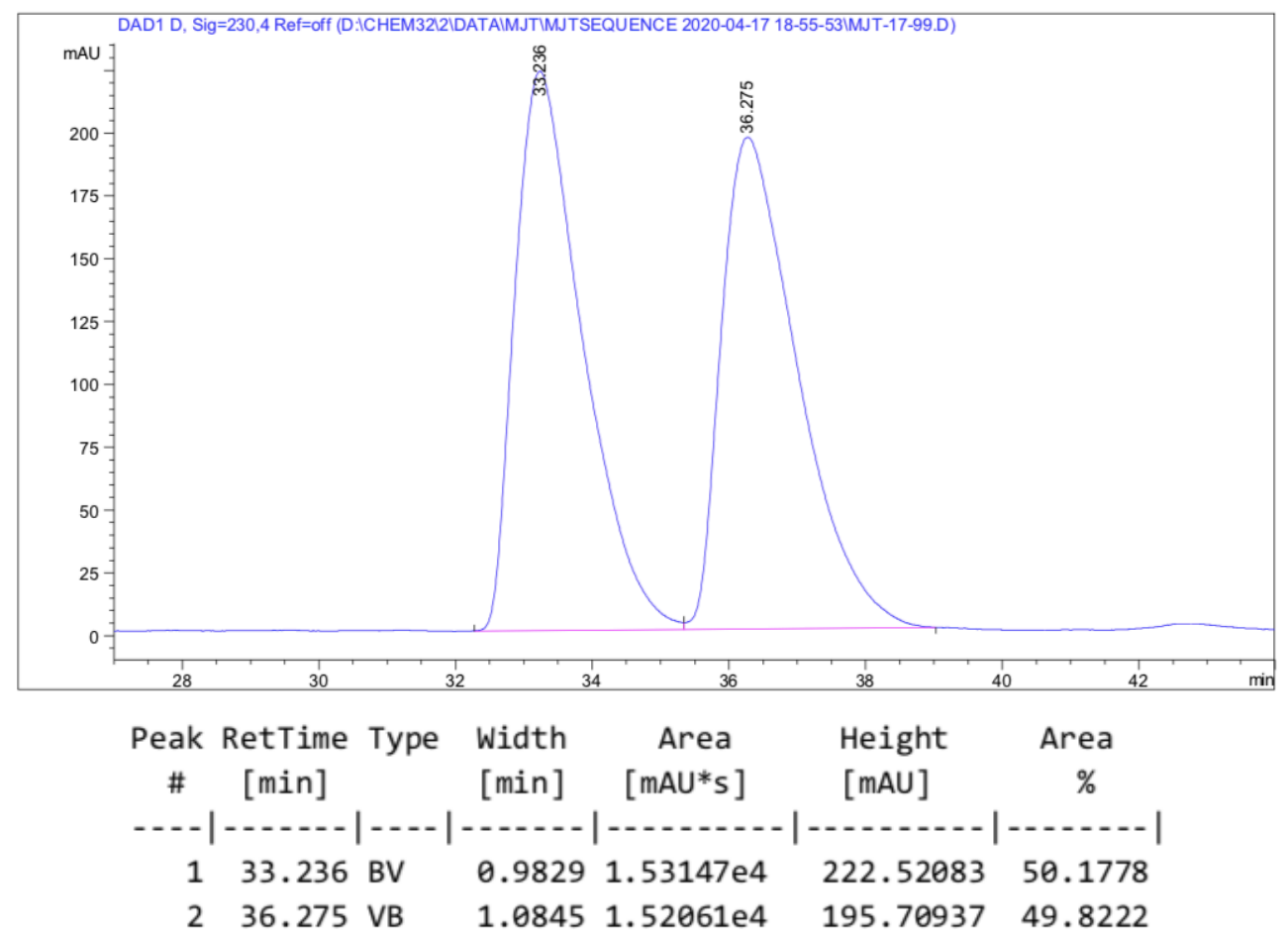

(S)-3,7-Dimethyl-3-vinyloct-6-enenitrile ((S)-3f)

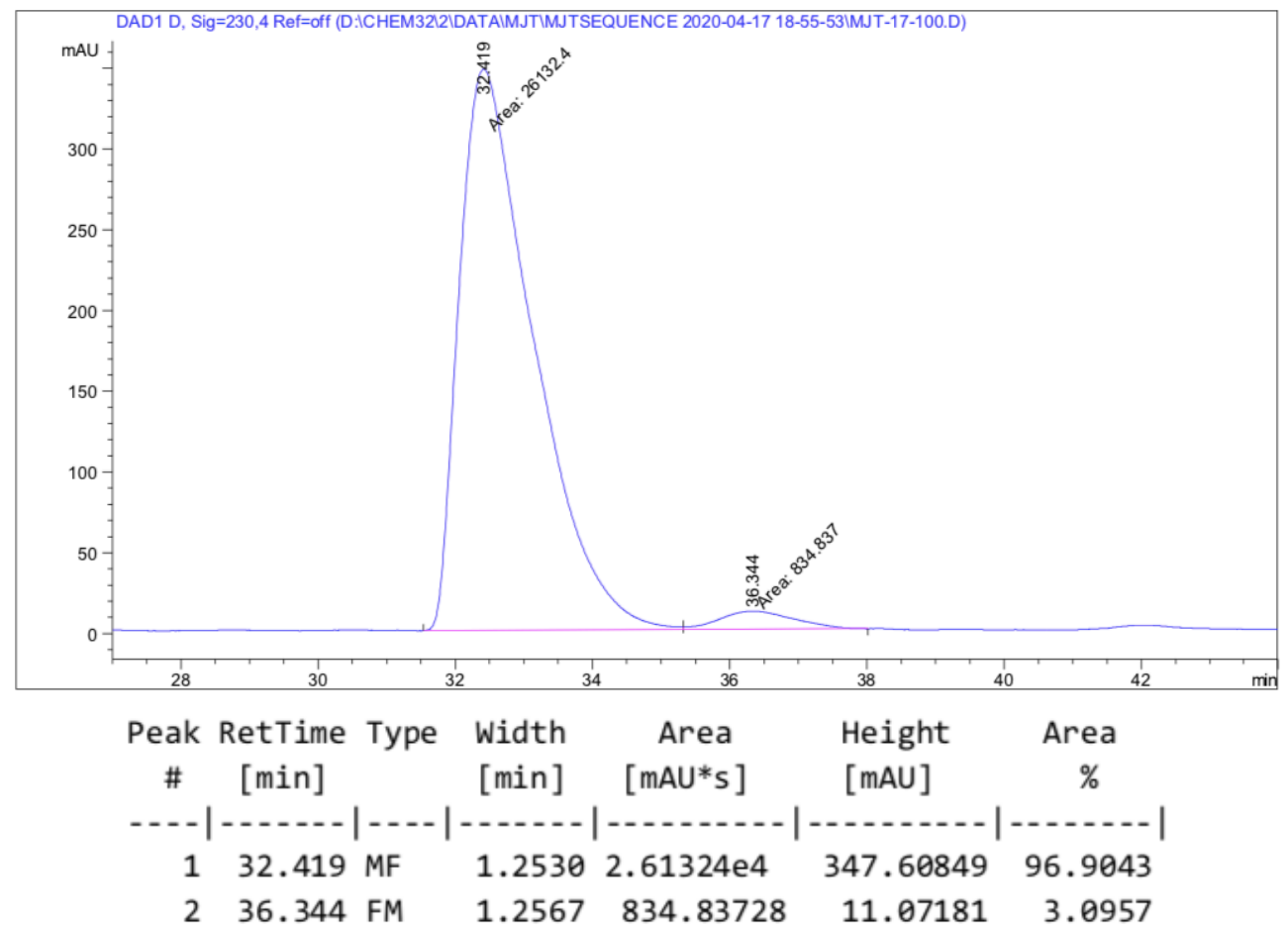


Methyl (2-(p-tolyl)but-3-en-2-yl) carbonate (2q)

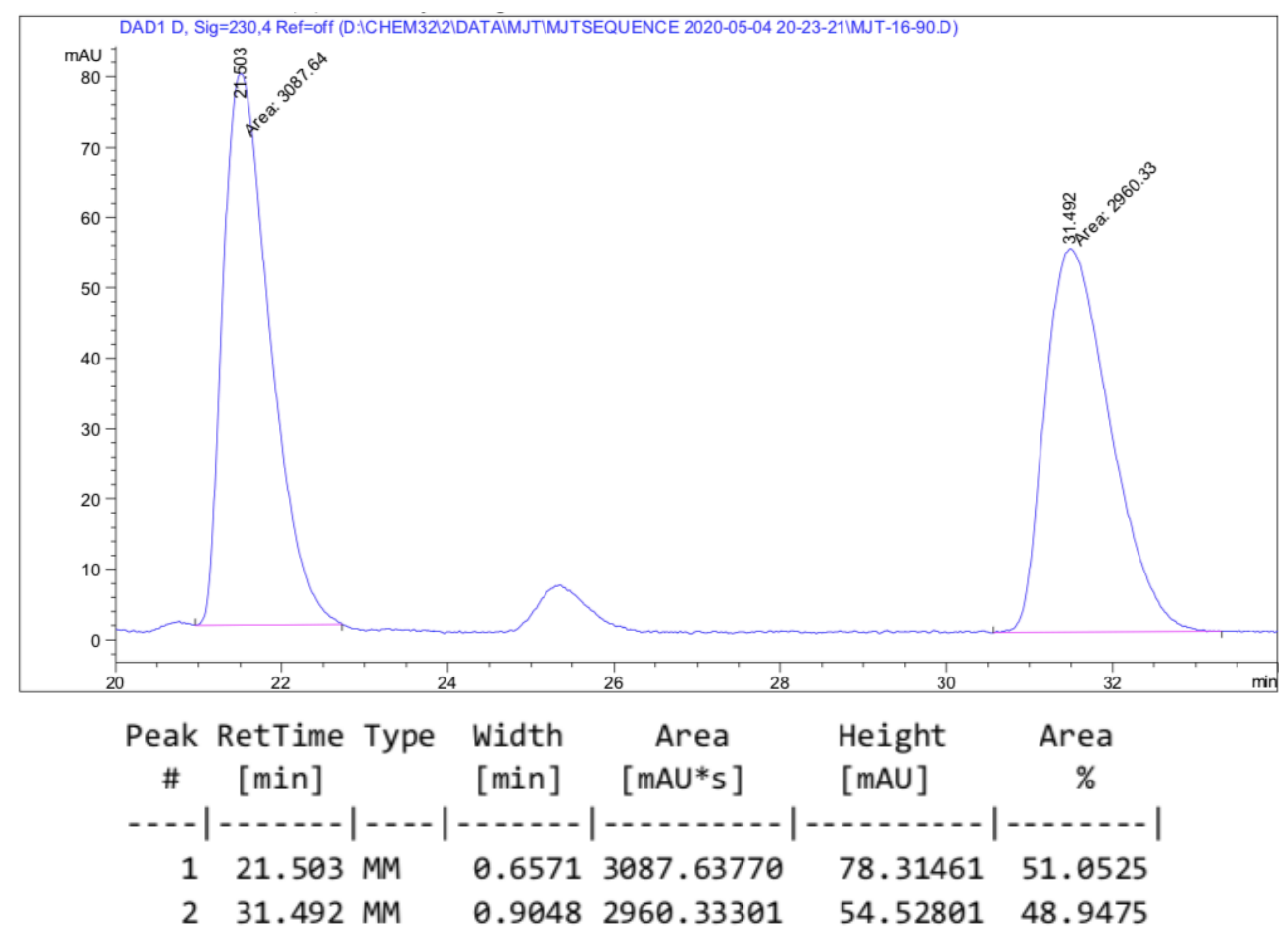

(R)-Methyl (2-(p-tolyl)but-3-en-2-yl) carbonate ((R)-2q)

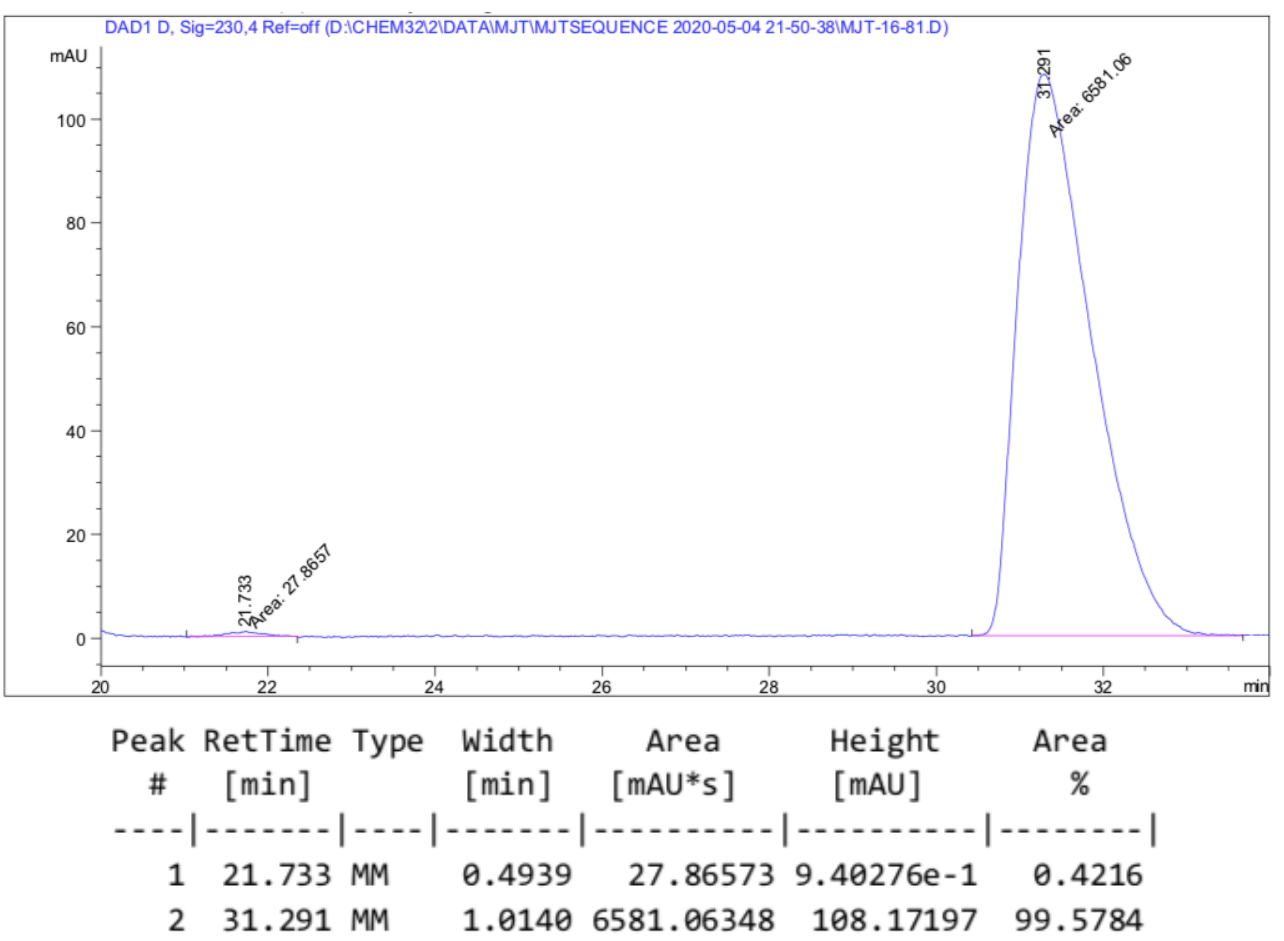




\section{3-Methyl-3-(p-tolyl)pent-4-enenitrile (3q)}

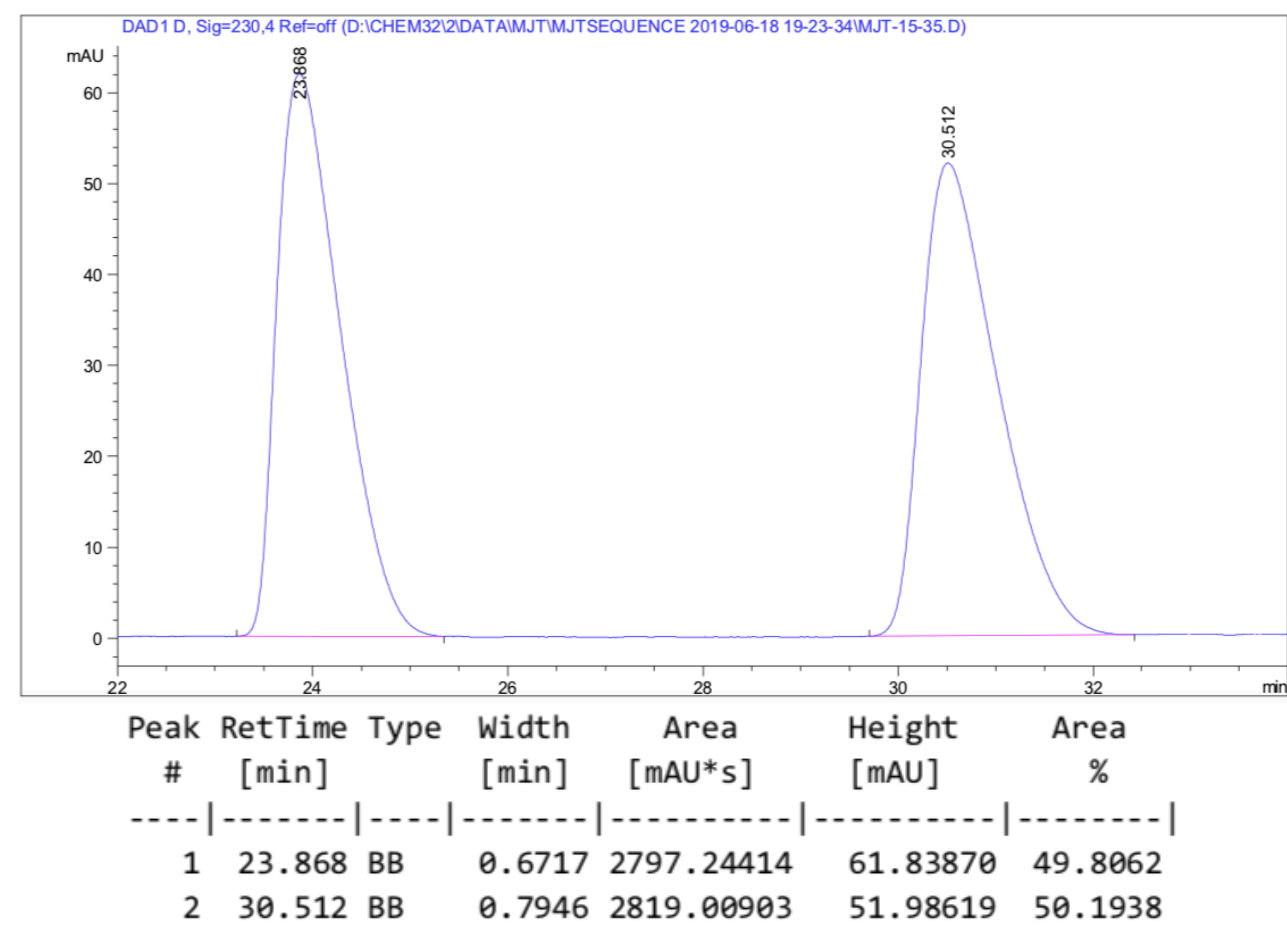

(R)-3-Methyl-3-(p-tolyl)pent-4-enenitrile ((R)-3q)

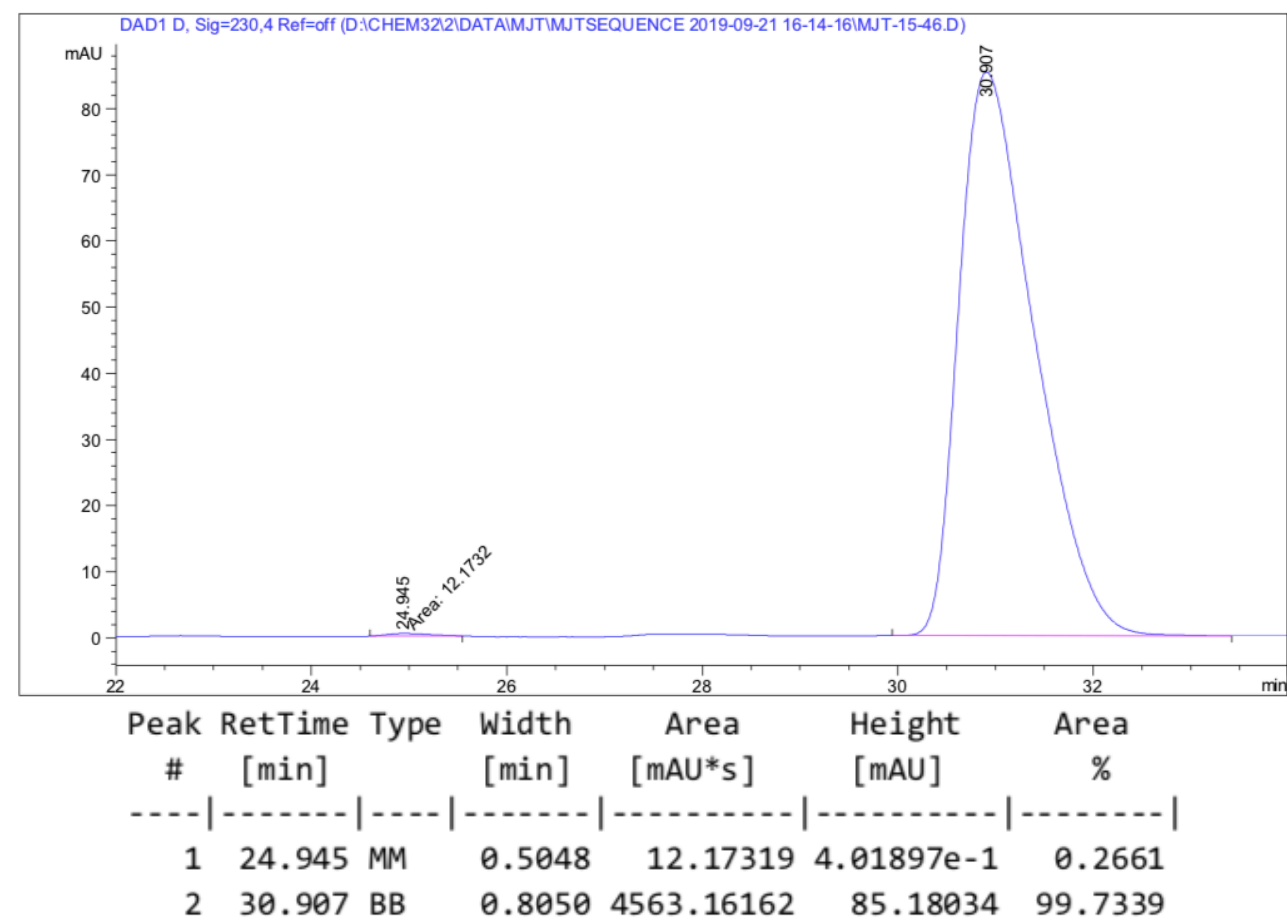




\section{3-Methyl-4-(p-tolyl)hex-5-en-2-one ( $r a c-5)$}

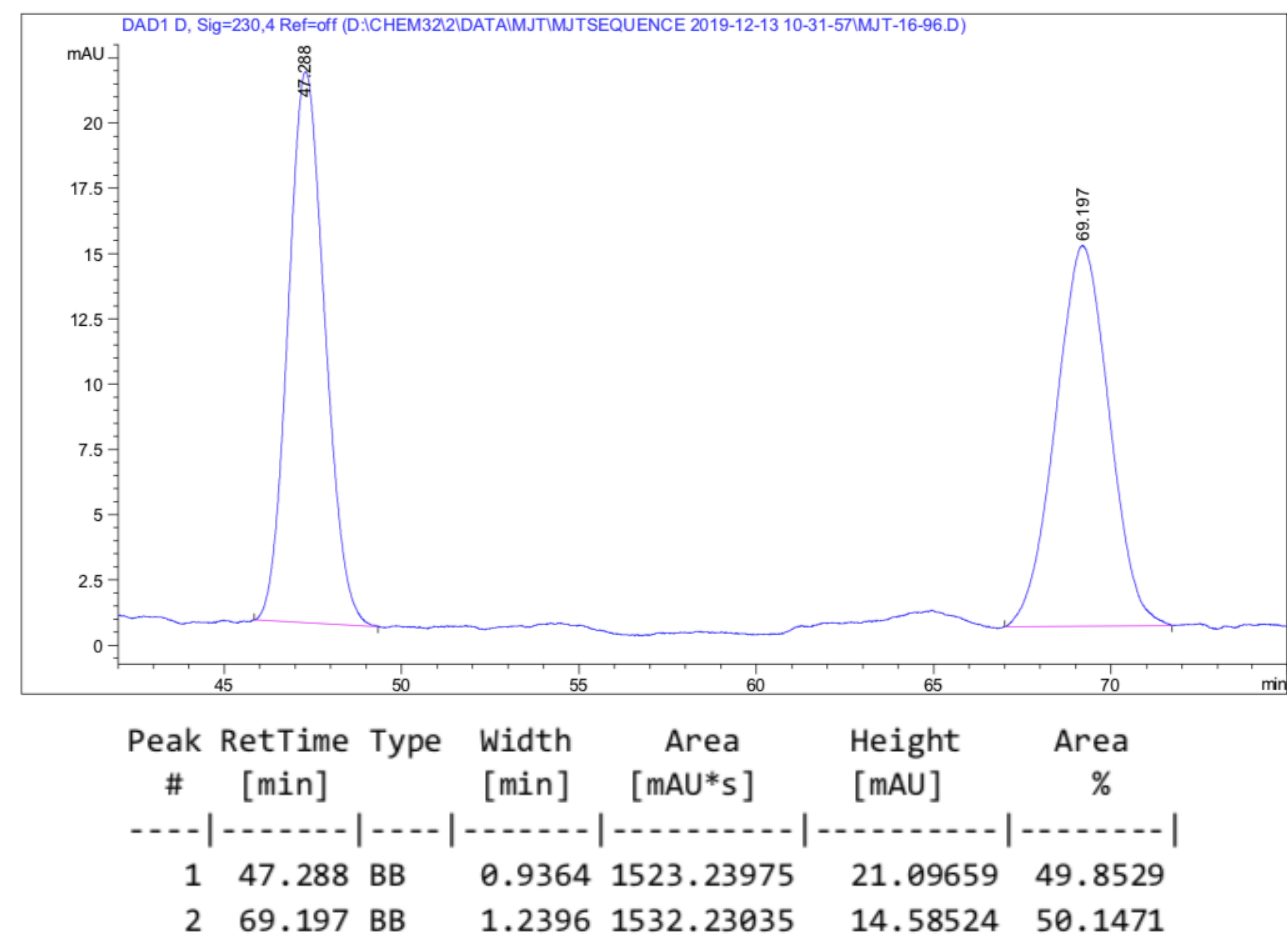

\section{(R)-4-Methyl-4-(p-tolyl)hex-5-en-2-one (5)}

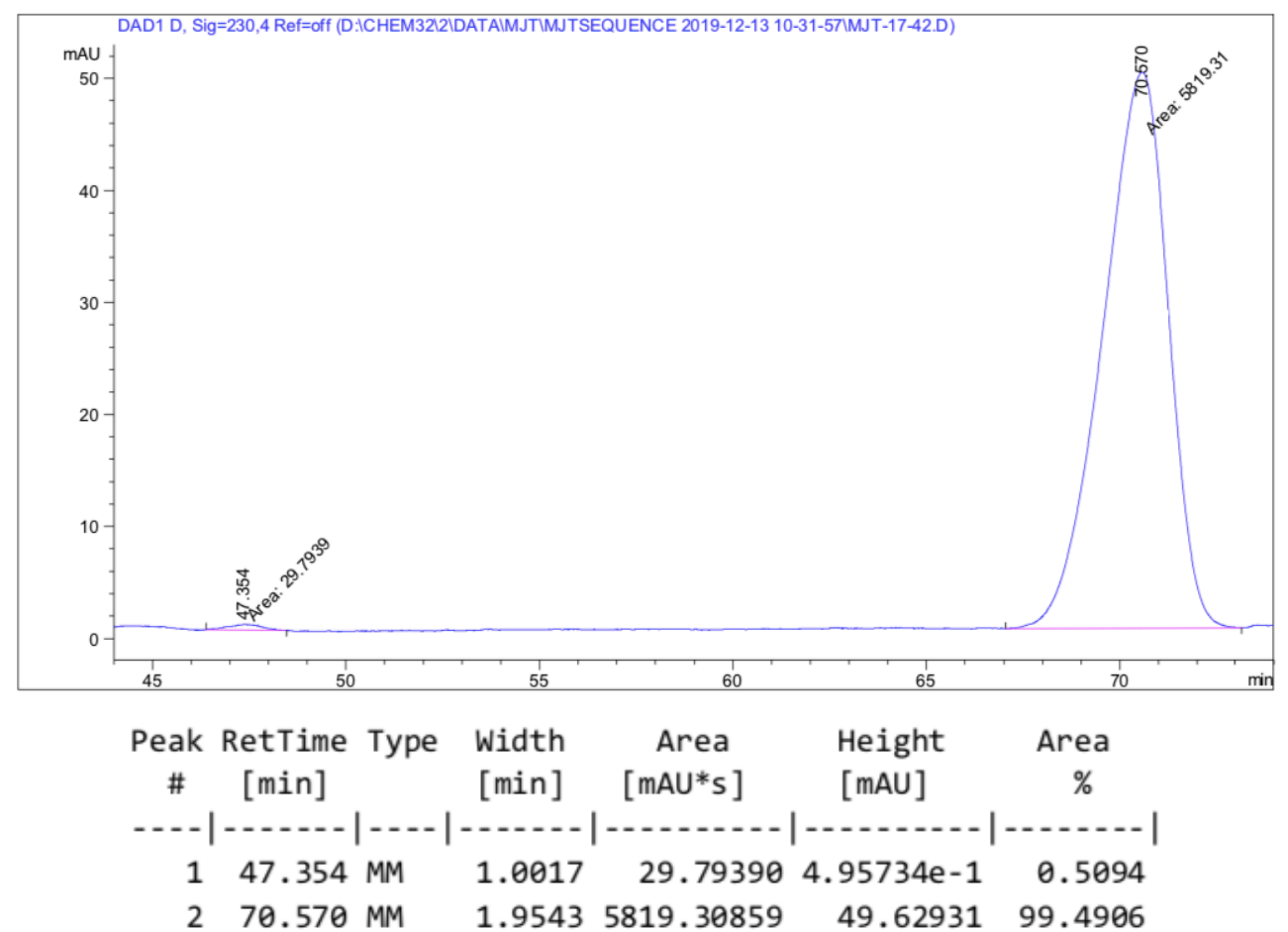


3-Methyl-3-(p-tolyl)pent-4-enamide ( rac-6)

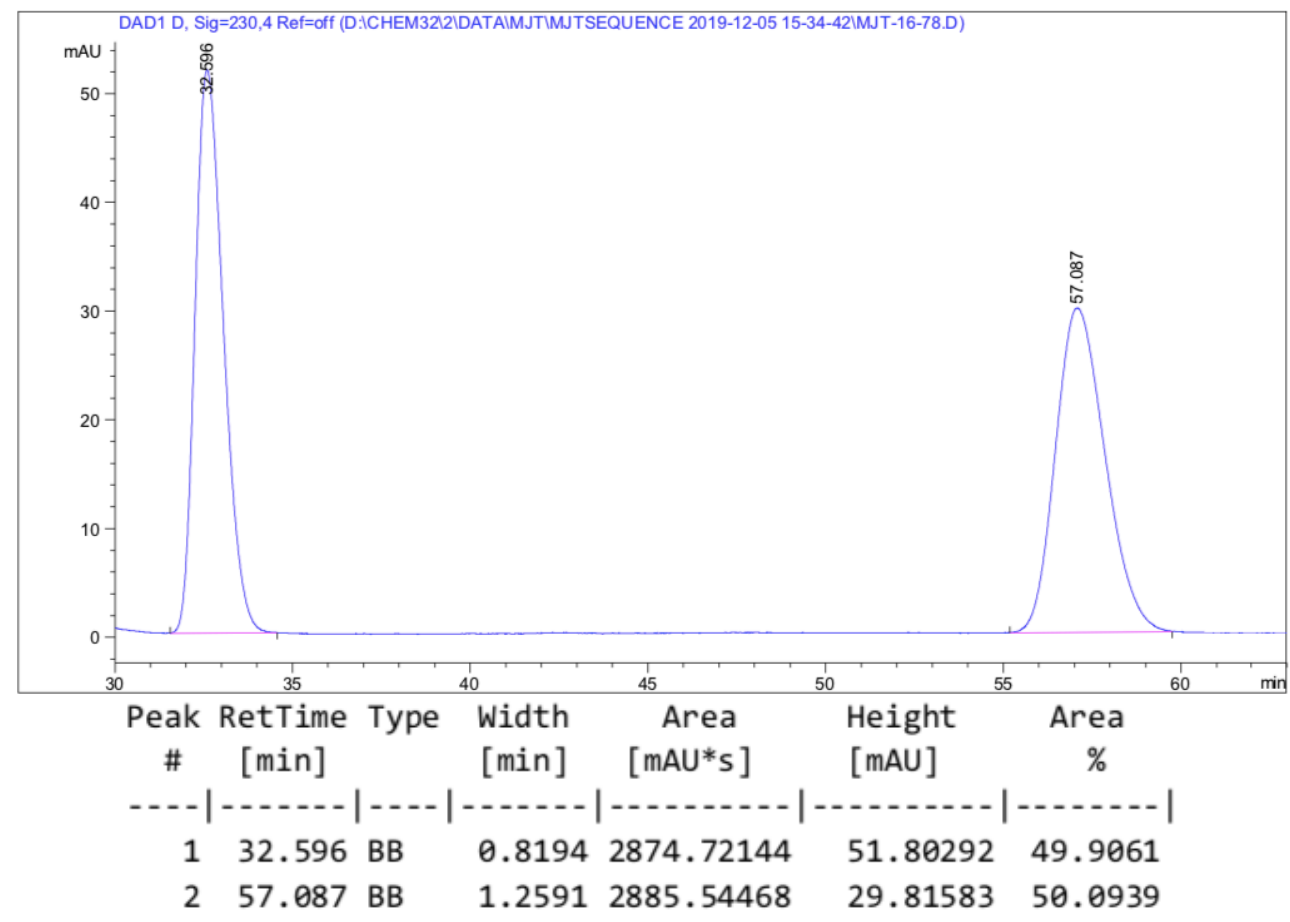

(R)-3-Methyl-3-(p-tolyl)pent-4-enamide (6)

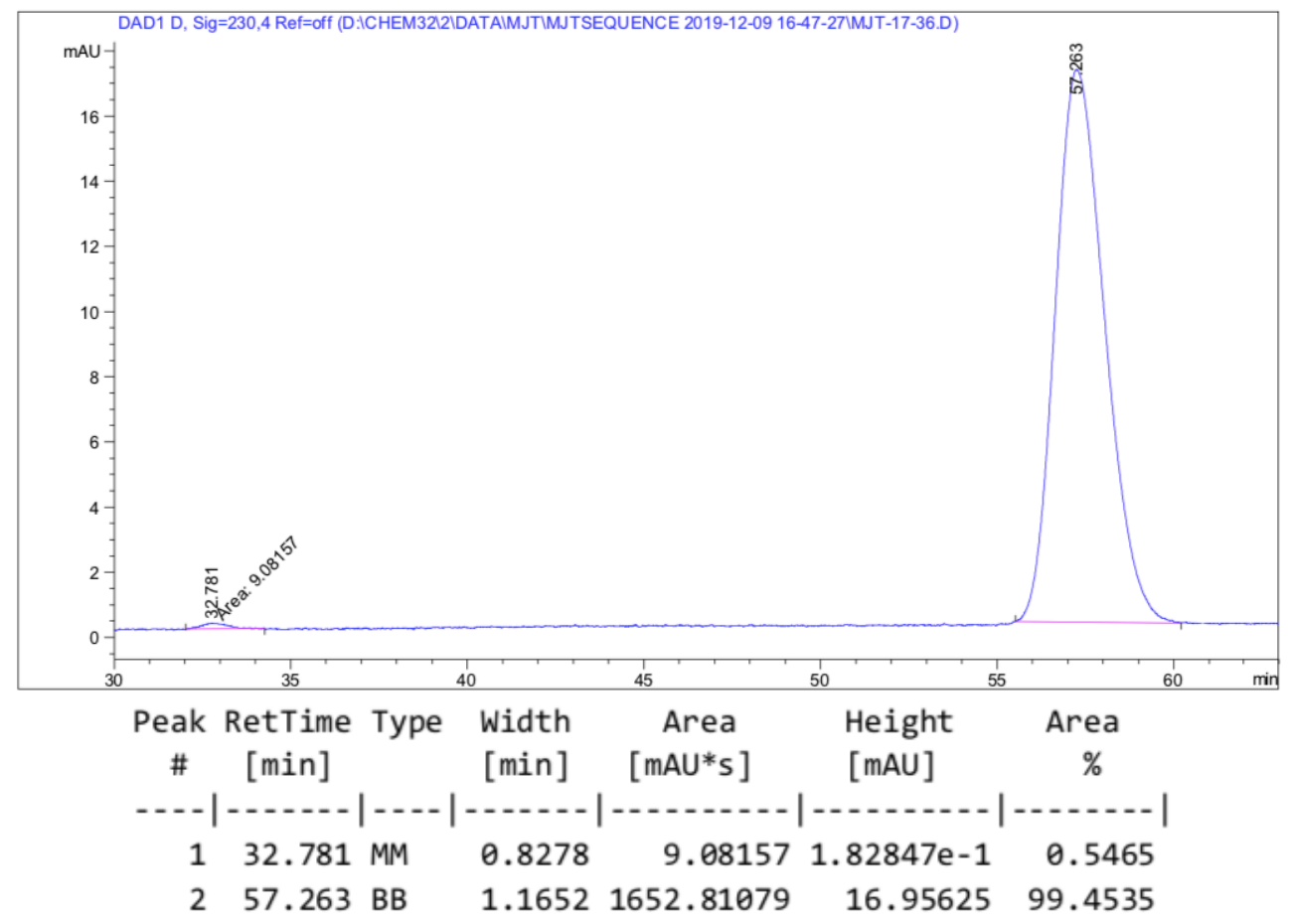




\section{3-Methyl-3-(p-tolyl)pent-4-enal ( $r a c-7)$}

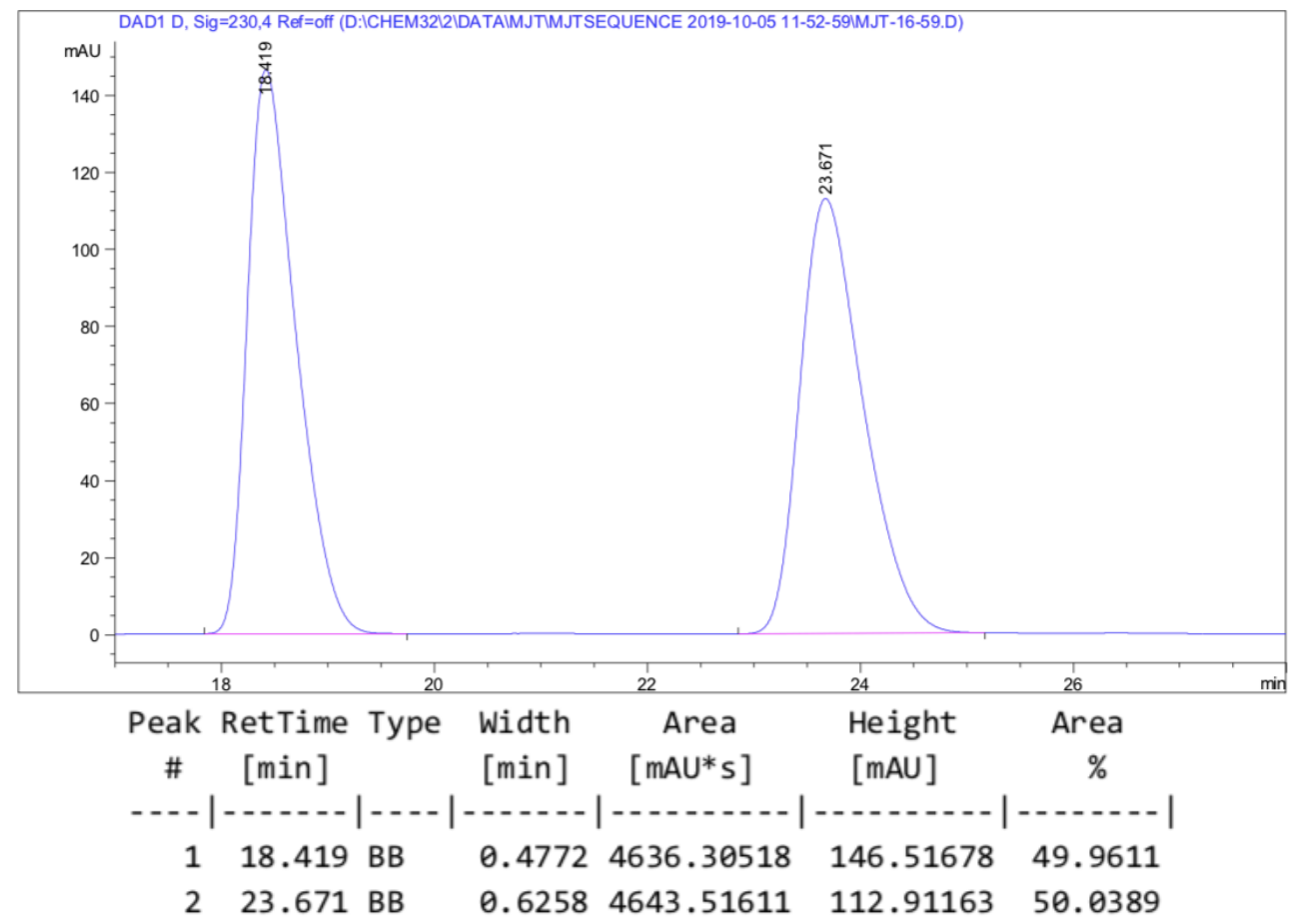

(R)-3-Methyl-3-(p-tolyl)pent-4-enal (7)

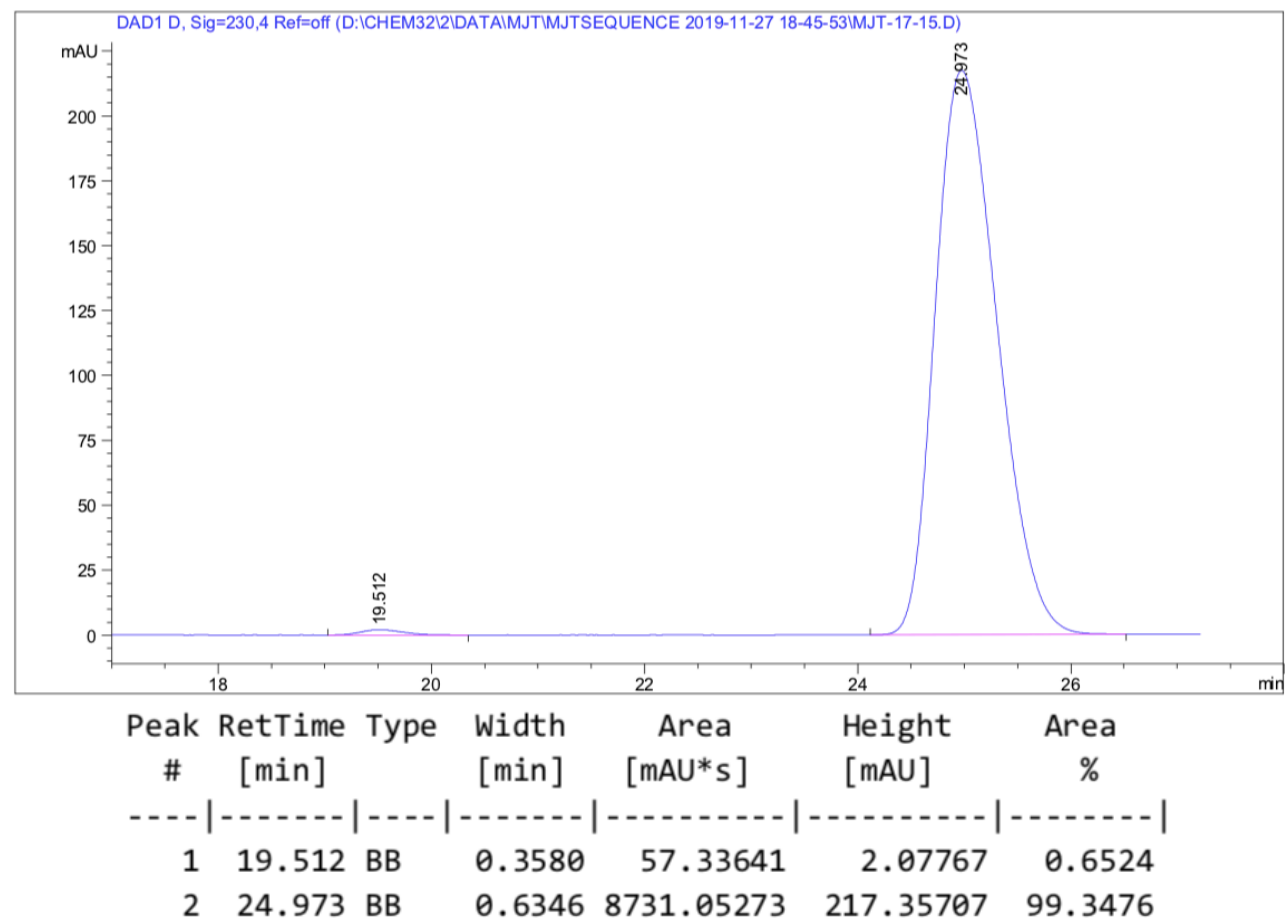

